\title{
A Comprehensive Assessment of Toxic Emissions From Coal-Fired Power Plants
}

\author{
Topical Report
}

\section{September 1996}

Work Performed Under Contract No.: DE-FC21-93MC30097

For

U.S. Department of Energy

Office of Fossil Energy

Morgantown Energy Technology Center

P.O. Box 880

Morgantown, West Virginia 26507-0880

By

University of North Dakota

Energy \& Enviromental Research Center

15 North 23rd Street

P. O. Box 9018

Grand Forks, North Dakota 58202-9018 


\section{Disclaimer}

This report was prepared as an account of work sponsored by an agency of the United States Government. Neither the United States Government nor any agency thereof, nor any of their employees, makes any warranty, express or implied, or assumes any legal liability or responsibility for the accuracy, completeness, or usefulness of any information, apparatus, product, or process disclosed, or represents that its use would not infringe privately owned rights. Reference herein to any specific commercial product, process, or service by trade name, trademark, manufacturer, or otherwise does not necessarily constitute or imply its endorsement, recommendation, or favoring by the United States Government or any agency thereof. The views and opinions of authors expressed herein do not necessarily state or reflect those of the United States Government or any agency thereof. 


\section{DISCLAIMER}

Portions of this document may be illegible electronic image products. Images are produced from the best available original document. 


\section{DISCLAIMER}

LEGAL NOTICE: This report was prepared by the Energy \& Environmental Research Center (EERC), an agency of the University of North Dakota, as an account of work sponsored by the U.S. Department of Energy, an agency of the United States Government. Neither the EERC nor the United States Government, nor any agency thereof, nor any of its employees makes any warranty, express or implied, or assumes any legal liability or responsibility for the accuracy, completeness, or usefulness of any information, apparatus, product, or process disclosed, or represents that its use would not infringe privately owned rights. Reference herein to any specific commercial product, process, or service by trade name, trademark, manufacturer, or otherwise does not necessarily constitute or imply its endorsement, recommendation, or favoring by the EERC or the United States Government or any agency thereof. The views and opinions of authors expressed herein do not necessarily state or reflect those of the United States Government or any agency thereof.

\section{ACKNOWLEDGMENTS}

The Energy \& Environmental Research Center (EERC) would like to express its appreciation to the U.S. Department of Energy Pittsburgh and Morgantown Energy Technology Centers (DOE PETC and DOE METC) for supporting this review effort. Also, the EERC would like to thank the representatives of the various prime contractors for providing clarification and/or answers to numerous questions raised during the review process. These include Barry Jackson (Roy F. Weston, Inc.), P. Vann Bush (Southern Research Institute), George Sverdrup (Battelle Columbus Operations), O.W. Hargrove (Radian Corp.), and Glenn C. England (Energy and Environmental Research Corporation). The EERC Project Manager would like to specifically thank Thomas D. Brown (DOE PETC) for his support and input concerning the review activity.

The EERC Project Manager, Stanley J. Miller, would like to thank his EERC coauthors for their efforts in completing the review activity and contributing to the preparation of this subtask report. The authors gratefully acknowledge the efforts of the other review team members: Michael E. Collings, John Erjavec, Huichong C. LeNore, and Jeffrey S. Thompson and EERC support staff who were instrumental in the preparation of this document. 
TABLE OF CONTENTS

LIST OF FIGURES $\ldots \ldots \ldots \ldots \ldots \ldots \ldots \ldots \ldots \ldots \ldots \ldots \ldots$ iii

LIST OF TABLES $\ldots \ldots \ldots \ldots \ldots \ldots \ldots \ldots \ldots \ldots \ldots \ldots \ldots \ldots$

GLOSSARY OF TERMS AND DEFINITIONS $\ldots \ldots \ldots \ldots \ldots \ldots \ldots \ldots$

EXECUTIVE SUMMARY $\ldots \ldots \ldots \ldots \ldots \ldots \ldots \ldots \ldots \ldots \ldots \ldots \ldots$

Introduction .......................... ES-1

Description of Field Test Program . . . . . . . . . . . . . . ES-3

Results ........................... ES-5

Round-Robin Coal Analyses . . . . . . . . . . . . . . . ES-6

Trace Element Emission Factors . . . . . . . . . . . . . . . . ES-8

Organic Emission Factors . . . . . . . . . . . . . . . ES-11

Radionuclide Emission Factors . . . . . . . . . . . . . . E ES-13

Acid Gas-Halogen Emission Factors . . . . . . . . . . . . . . . . . ES-14

Estimates of Total HAP Emissions . . . . . . . . . . . . . ES-15

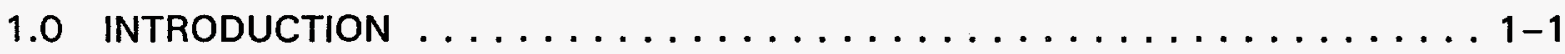

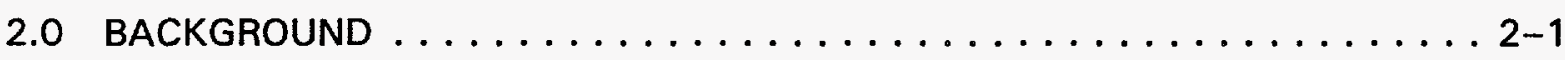

3.0 DESCRIPTION OF FIELD TEST PROGRAM $\ldots \ldots \ldots \ldots \ldots \ldots \ldots \ldots .1$

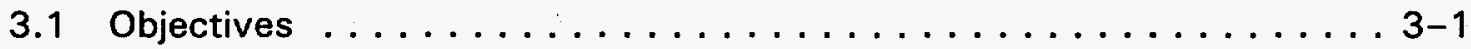

3.2 Description of Plants Sampled . . . . . . . . . . . . . 3-3

3.3 Description of Sampling, Analytical, and QA/OC Methods . . . . . . 3-6

4.0 RESULTS AND DISCUSSION OF PHASE I DATA $\ldots \ldots \ldots \ldots \ldots \ldots$. $\ldots \ldots \ldots$

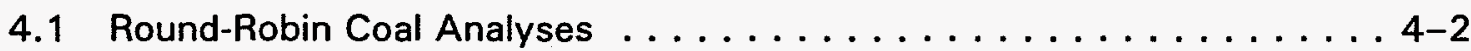

4.1.1 Scope of Round-Robin Program . . . . . . . . . . . 4-3

4.1.2 Contractors' Results .................. 4-4

4.1 .3 Accuracy ...................

4.1.4 Reproducibility $\ldots \ldots \ldots \ldots \ldots \ldots \ldots \ldots \ldots \ldots \ldots$ 4-9

4.1.5 Repeatability .................... 4-11

4.1.6 Discussion: Comparison of Feed Coals .......... 4-11

4.2 Trace Element Emission Factors . . . . . . . . . . . . . 4-13

4.2.1 Criteria for Assessing Organic and Inorganic Data Quality . . 4-17

4.2.2 Mercury Speciation and Emission Factors ......... 4-21

4.2.3 Other Volatile Trace Elements . . . . . . . . . . . . . . . 4-24

4.2.4 Nonvolatile Trace Elements . . . . . . . . . . . . . . 4-29

4.3 Organic Emission Factors . . . . . . . . . . . . . . . . . 4-38

4.3.1 Aldehydes and Ketones . . . . . . . . . . . . 4-40

4.3.2 Volatile Organic Compounds ............... 4-41

4.3.3 Semivolatile Organic Compounds and Polycyclic Aromatic

Hydrocarbons . . . . . . . . . . . . . . 4-42 
4.3.4 Chlorinated Dioxins and Furans ... . . . . . . . . . . . 4-43

4.4 Radionuclide Emission Factors . . . . . . . . . . . . . . . . . . . . 4-44

4.5 Acid Gases-Halogen Emission Factors . . . . . . . . . . . . . . . . . 4-46

4.6 Discussion of Special Topics ... . . . . . . . . . . . . . . 4-51

4.6.1 Plume-Simulating Dilution Sampler . . . . . . . . . . . . 4-51

4.6.2 Trace Element Enrichment as a Function of Particle Size . . . 4-53

4.6.3 Particle-Size Distributions of Stack Emissions and

FF/ESP Hopper Ash . . . . . . . . . . . . . . . . 4-55

4.6.4 Chromium Speciation and Sampling . . . . . . . . . . . . 4-57

4.6.5 Dioxins-Furans Across ESPs . . . . . . . . . . . . . 4-59

4.6.6 Effect of Sootblowing on Trace Element Emissions . . . . . . 4-60

4.6.7 HAPs on Particle Surfaces . . . . . . . . . . . . . . 4-62

5.0 CONCLUSIONS AND RECOMMENDATIONS $\ldots \ldots \ldots \ldots \ldots \ldots \ldots \ldots \ldots$

5.1 Round-Robin Coal Analyses . . . . . . . . . . . . . . . . 5-1

5.2 Organic Emission Factors . . . . . . . . . . . . . . . . 5-2

5.3 Trace Element Emission Factors . . . . . . . . . . . . . . . . 5-3

5.4 Radionuclide Emission Factors . . . . . . . . . . . . . . . . 5 5-5

5.5 Acid Gases-Halogen Emission Factors . . . . . . . . . . . . . . . 5-5

5.6 Special Topics . . . . . . . . . . . . . . . . . . . . . 5-5

5.6.1 Simulated Plume Dilution Sampling . . . . . . . . . . 5-5

5.6.2 Trace Element Enrichment as a Function of Particle Size . . . . . 5-6

5.6.3 Particle-Size Distribution of Fly Ash and Stack Emissions . . . 5-6

5.6.4 Chromium Speciation and Sampling ............ . 5-6

5.6.5 Dioxins-Furans Across ESPs ... . . . . . . . . . . . 5-7

5.6.6 Effect of Sootblowing on Trace Element Emissions . . . . . . . 5-7

5.6.7 HAPs on Particle Surfaces . . . . . . . . . . . . . 5-8

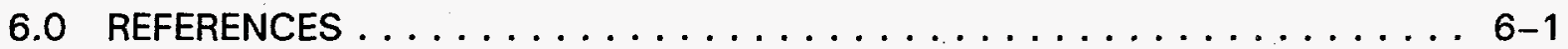

INORGANIC DATA FOR STACK GAS SAMPLES $\ldots \ldots \ldots \ldots \ldots$ Appendix A

ORGANIC DATA FOR STACK GAS SAMPLES $\ldots \ldots \ldots \ldots \ldots \ldots$ Appendix B

RADIONUCLIDE DATA FOR COAL AND STACK GAS SAMPLES . . . . . A Appendix C 


\section{LIST OF FIGURES}

ES-1 DOE Phase I Stack Concentration Data for 11 Trace Elements and Four Acid Gases Compared to EPA Ambient Air Data and Quality Standards . ES-9

ES-2 Emission Factors for 16 Trace Elements at Each of Nine DOE Phase I Field Process Configurations . . . . . . . . . . . . . . . E ES-9

ES-3 Summary of Estimated Annual HAP Emissions for the Nine DOE Phase I Process Configurations Including Stack Particulate Mass Loading $\left(\mathrm{mg} / \mathrm{Nm}^{3}\right)$ and Particulate Removal Efficiency . . . . . . . . . . . ES-18

4-1 Interlaboratory Variability by Trace Element . . . . . . . . . . . 4-10

4-2 Correlation of Trace Element Analytical Variability with As-Determined Heating Value ........................ . 4-10

4-3 Average Intralaboratory Repeatability for Trace Elements . . . . . . 4-11

4-4 Classification of Selected Elements Relative to Their Volatility and Partitioning in Power Plants . . . . . . . . . . . . . . . . . . 4-14

4-5 Emission Factors for 16 Trace Elements at Each of Nine DOE Phase I Process Configurations . . . . . . . . . . . . . . . . 4-15

4-6 DOE Phase I Stack Concentration Data for 11 Trace Elements and Four Acid Gases Compared to EPA Ambient Air Data and Quality Standards ... . . . . . . . . . . . . . . . . . . . 4-17

4-7 Emission Factor and Penetration Data for Mercury . . . . . . . . . 4-23

4-8 Emission Factor and Penetration Data for Boron . . . . . . . . . . 4-26

4-9 Emission Factor and Penetration Data for Selenium . . . . . . . . 4-28

4-10 Emission Factor and Penetration Data for Antimony ........ . . 4-30

4-11 Emission Factor and Penetration Data for Arsenic . . . . . . . . . . . 4-30

4-12 Emission Factor and Penetration Data for Barium . . . . . . . . . 4-31

4-13 Emission Factor and Penetration Data for Beryllium . . . . . . . . 4-31

4-14 Emission Factor and Penetration Data for Cadmium . . . . . . . . 4-32

4-15 Emission Factor and Penetration Data for Chromium . . . . . . . . . 4-32

4-16 Emission Factor and Penetration Data for Cobalt $\ldots \ldots \ldots \ldots \ldots$. . . 4-33 
4-17 Emission Factor and Penetration Data for Copper . . . . . . . . . 4 4-33

4-18 Emission Factor and Penetration Data for Lead . . . . . . . . . . . . . 4-34

4-19 Emission Factor and Penetration Data for Manganese . . . . . . . . . 4-34

4-20 Emission Factor and Penetration Data for Molybdenum . . . . . . . . . 4-35

4-21 Emission Factor and Penetration Data for Nickel . . . . . . . . . . . . 4-35

4-22 Emission Factor and Penetration Data for Vanadium . . . . . . . . 4-36

4-23 Emission Factor and Penetration Data for Total Chlorine . . . . . . . . . 4-47

4-24 Emission Factor and Penetration Data for Total Fluorine . . . . . . . . . 4-48

4-25 Trace Element Enrichment Factor, Comparison of Largest and Smallest Particle-Size Fractions . . . . . . . . . . . . . . . . . . . . . . 4-54

4-26 Flue Gas Particulate Sample Preparation and Analysis Plan for

Extractable Metals . . . . . . . . . . . . . . . . . . . . . . . . 4-63

4-27 Sb Content of Flue Gas Particulate as Sampled at Plant Yates . . . . . . 4-64

4-28 As Content of Flue Gas Particulate as Sampled at Plant Yates . . . . . . 4-64

4-29 Ba Content of Flue Gas Particulate as Sampled at Plant Yates . . . . . 4-65

4-30 Be Content of Flue Gas Particulate as Sampled at Plant Yates . . . . . 4-65

4-31 Cd Content of Flue Gas Particulate as Sampled at Plant Yates . . . . . 4-66

4-32 Cr Content of Flue Gas Particulate as Sampled at Plant Yates . . . . . . 4-66

4-33 Co Content of Flue Gas Particulate as Sampled at Plant Yates . . . . . 4-67

4-34 Cu Content of Flue Gas Particulate as Sampled at Plant Yates . . . . . 4-67

4-35 Pb Content of Flue Gas Particulate as Sampled at Plant Yates . . . . . 4-68

4-36 Mn Content of Flue Gas Particulate as Sampled at Plant Yates . . . . . 4-68

4-37 Mo Content of Flue Gas Particulate as Sampled at Plant Yates . . . . . 4-69

4-38 Ni Content of Flue Gas Particulate as Sampled at Plant Yates . . . . . 4 4-69

4-39 Se Content of Flue Gas Particulate as Sampled at Plant Yates . . . . . 4-70

4-40 V Content of Flue Gas Particulate as Sampled at Plant Yates . . . . . . 4-70 


\section{LIST OF TABLES}

ES-1 A Summary of DOE Phase I Hazardous Air Pollutant Emission

Factor Data . . . . . . . . . . . . . . . . . . . ES-16

3-1 Field Site Descriptions for DOE Phase I HAP Sampling . . . . . . . . . 3-4

3-2 Sample Types and Locations Generic to the HAP Assessment Effort . . . 3-6

3-3 Critical Target Analytes for Which Sampling and Analysis Were

Specifically Requested . . . . . . . . . . . . . . . . . 3-8

3-4 Analytical Techniques Used to Detect and Quantify Critical Target

Analytes ............................. . . . . . . . . .

3-5 Sampling and Analytical Contractors and Subcontractors . . . . . . 3-10

3-6 HAPs Listed in the 1990 CAAA . . . . . . . . . . . . . 3-11

4-1 Parameters Tested in the Round-Robin Study . . . . . . . . . . . 4-2

4-2 Fuels Included in the Round-Robin Study . . . . . . . . . . 4-3

4-3 Plant Results for Each Coal: Dry Coal Proximate-Ultimate and

Major Element Analyses . . . . . . . . . . . . . . . . . . . 4-5

4-4 Plant Results for Each Coal: Dry Coal Trace Element Analyses . . . . . 4-5

4-5 Percent Difference Between Plant and Round-Robin Results for

Proximate-Ultimate and Major Element Analyses . . . . . . . . . . . . 4-6

4-6 Percent Difference Between Plant and Round-Robin Results for

Trace Element Analyses . . . . . . . . . . . . . . . . . . . 4-6

4-7 Round-Robin Results for Each Coal: Dry Coal Proximate-Ultimate and Major Element Analyses . . . . . . . . . . . . . . . 4-7

4-8 Round-Robin Results for Each Coal: Dry Coal Trace Element Analyses . . 4-7

4-9 Accuracy of Round-Robin Results for NIST SRM $1632 b \ldots \ldots$. . . . . 4-8

4-10 Coal Trace Element Content in $\mathrm{lb} / 10^{12}$ Btu (dry basis) Based on

Average Round-Robin Results .................. 4-12

4-11 Proposed HAP Data Reporting Format . . . . . . . . . . . . . . . . 4-19

4-12 Summary of Organic Emissions . . . . . . . . . . . . . 4-41

4-13 Summary of Radionuclide Results for Nine Coal-Fired Utility Process

Configurations ....................... . . 4-45 
4-14 Mass Median Aerodynamic Diameter at Control Device Inlet and Stack . 4-56

4-15 Average Stack Concentrations, Field Blanks, and Emission Factors of Hexavalent Chromium . . . . . . . . . . . . . . . . . . 4-59

4-16 Average Extractability of Elements in Fly Ash . . . . . . . . . . 4-71 


\section{GLOSSARY OF TERMS AND DEFINITIONS}
$\mathrm{A} / \mathrm{C}$
air-to-cloth ratio
AFGD
advanced flue gas desulfurization
ASTM
American Society for Testing and Materials
Btu
British thermal unit
CAAA
Clean Air Act Amendments
$\mathrm{Cl}$ confidence interval
CV-AAS cold-vapor-atomic absorption spectroscopy
CV-AF cold-vapor-atomic fluorescence
DGA-CVAA double-gold amalgam-cold-vapor atomic absorption
DL detection limit
DOE
Department of Energy, U.S.
EER Energy and Environmental Research Corporation
EERC Energy \& Environmental Research Center
EPA Environmental Protection Agency, U.S.
EPRI Electric Power Research Institute
ESP electrostatic precipitator
FF fabric filter
FGD flue gas desulfurization
GA-CVAA gold amalgam-cold-vapor atomic absorption
GC gas chromatography
GC-MS gas chromatography-mass spectroscopy
GF-AAS graphite furnace-atomic absorption spectroscopy
HAP hazardous air pollutant
HEST hazardous element sampling train
HpCdd heptachloro dibenzo-p-dioxins
HpCDF heptachloro dibenzofurans
HPLC high-performance liquid chromatography
IC ion chromatography
ICP-MS inductively coupled plasma-mass spectroscopy
ICP-AES inductively coupled plasma-atomic emission spectroscopy
IGCC integrated gasification combined cycle
INAA instrumental neutron activation analysis 


\begin{tabular}{|c|c|}
\hline JBR & jet bubbling reactor \\
\hline $\mathrm{lb} / 10^{12} \mathrm{Btu}$ & pound per trillion Btu \\
\hline LLQ & lower limit of quantitation \\
\hline MACT & maximum achievable control technology \\
\hline MESA & mercury speciation adsorption \\
\hline METC & Morgantown Energy Technology Center \\
\hline MMBtu & million Btu \\
\hline MMD & mass median diameter \\
\hline $\mathrm{MWe}$ & megawatt \\
\hline ND & nondetect \\
\hline NIST & National Institute of Standards and Technology \\
\hline $\mathrm{Nm}^{3}$ & normal cubic meter $10^{\circ} \mathrm{C}$ and 1 atmosphere) \\
\hline NTIS & National Technical Information Service \\
\hline OCDD & octachloro dibenzo-p-dioxins \\
\hline OCDF & octachloro dibenzofurans \\
\hline $\mathrm{PAH}$ & polycyclic aromatic hydrocarbon \\
\hline PCDD & polychlorinated dibenzo-p-dioxins \\
\hline PCDF & polychlorinated dibenzofurans \\
\hline $\mathrm{pCi} / \mathrm{g}$ & picocuries per gram \\
\hline PeCDF & pentachlorinated dibenzofurans \\
\hline PETC & Pittsburgh Energy Technology Center \\
\hline PFBC & pressurized fluid-bed combustion \\
\hline PISCES & EPRI Power Plant Integrated Systems: Chemical Emissions Study \\
\hline PRB & Powder River Basin \\
\hline PRSD & percent relative standard deviation \\
\hline PSD & percent standard deviation \\
\hline PSDS & plume-simulating dilution sampling \\
\hline QA/OC & quality assurance/quality control \\
\hline RCRA & Resource Conservation and Recovery Act \\
\hline RE & removal efficiency \\
\hline RFP & request for proposal \\
\hline RTI & Research Triangle Institute \\
\hline SCA & specific collection area \\
\hline
\end{tabular}




$\begin{array}{ll}\text { SCR } & \text { selective catalytic reduction } \\ \text { SD } & \text { standard deviation } \\ \text { SDA } & \text { spray dryer absorber } \\ \text { SIE } & \text { specific ion electrode } \\ \text { SIMS } & \text { selective ion monitoring spectroscopy } \\ \text { SRI } & \text { Southern Research Institute } \\ \text { SRM } & \text { standard reference material } \\ \text { SVOC } & \text { semivolatile organic compound } \\ \text { t } & \text { tangentially } \\ \text { TCDF } & \text { tetrachloro dibenzofurans } \\ \text { UARG } & \text { Utility Air Regulatory Group } \\ \text { VOCs } & \text { volatile organic compounds } \\ \text { VOST } & \text { volatile organic sampling train } \\ \text { XRF } & \text { x-ray fluorescence } \\ \mu \text { g/Nm } & \end{array}$




\title{
EXECUTIVE SUMMARY
}

\author{
INTRODUCTION
}

The 1990 Clean Air Act Amendments (CAAA) have two primary goals: pollution prevention and a market-based least-cost approach to emission control. To address air quality issues as well as permitting and enforcement, the 1990 CAAA contain 11 sections or titles. The individual amendment titles are as follows:

Title I - National Ambient Air Quality Standards

Title II - Mobile Sources

Title III - Hazardous Air Pollutants

Title IV - Acid Deposition Control

Title V - Permits

Title VI - Stratospheric Ozone Protection Chemicals

Title VII - Enforcement

Title VIII - Miscellaneous Provisions

Title IX - Clean Air Research

Title X - Disadvantaged Business Concerns

Title XI - Clean Air Employment Transition Assistance

Titles I, III, IV, and V will change or have the potential to change how operators of coal-fired utility boilers control, monitor, and report emissions. For the purpose of this discussion, Title III is the primary focus.

Title III, Hazardous Air Pollutants (HAPs), requires the U.S. Environmental Protection Agency (EPA) to establish stationary source categories and to implement regulatory standards for 189 air toxics from source categories emitting 25 tons annually of any combination of pollutants or 10 tons annually of a single pollutant. In addition, EPA must issue maximum achievable control technology (MACT) standards. The original list of 189 HAPs may be expanded or reduced based on risk to public health, and once controls are in place, residual risk assessments must be performed to 
determine whether further reductions are needed. Although this amendment requires the regulation of commercial, industrial, and municipal sources, it does not specifically require the regulation of HAPs from utility boilers. Rather, Title III requires that EPA study HAP emissions from utility boilers to determine potential health effects prior to promulgating any new regulations. In addition, a study of mercury emissions from utility steam generators, municipal waste combustion units, and other sources was mandated.

In response to the 1990 CAAA, the U.S. Department of Energy (DOE) is participating in a collaborative effort with the Utility Air Regulatory Group (UARG), EPA, and the Electric Power Research Institute (EPRI) to establish information upon which future regulatory activities can be based. The field sampling efforts are being led primarily by DOE and EPRI, with a few utility companies generating data for their specific systems. EPRI and DOE have provided most of their collected data to EPA.

The DOE approach to development of a HAP emission database for fossil fuel-fired utility systems has been twofold. The Morgantown Energy Technology Center (METC) has funded HAP-sampling activities at two advanced power system demonstration sites. These sites represent advanced combustion and gasification technologies and associated gas stream cleanup strategies. The Pittsburgh Energy Technology Center (PETC) has focused on establishing a database for current coal-fired systems, including conventional and advanced emission control technologies. PETC issued a request for proposals (RFP No. DE-RP22-92PC91349) entitled "Comprehensive Assessment of Toxic Emissions from Coal-Fired Power Plants" on February 10, 1992. As a result, PETC awarded Phase I contracts to five organizations for HAP sampling at eight utility sites representing nine process configurations. The purpose of the field sampling activities was to document the types and concentrations of potential HAPs from a select group of utility stations representing a cross section of U.S. coal-fired utility boilers. Utility station information is detailed in Table 3-1. Sampling activities were initiated in 1993, and final project reports were prepared in 1994.

The University of North Dakota Energy \& Environmental Research Center (EERC), at the request of PETC, reviewed the contractor reports documenting the results from 
completed sampling activities. The EERC objective was to provide an overview of the important findings from the Phase I air toxics assessment. This document summarizes key results from the nine contractor reports with an emphasis on stack-sampling data.

\section{Description Of Field Test Program}

The PETC field test program is focused on generating HAP data for coal-fired utility systems. The effort consists of two phases. Phase I of the HAP assessment program evaluated HAP emissions from eight coal-fired plants representing nine process configurations. Phase II was intended to be an option, based on Phase I results, to be exercised by PETC in the event that additional or similar plant configurations were selected for sampling. At this time, Phase II activities are in progress. One plant is an integrated gasification combined-cycle (IGCC) system; three of the plants represent more conventional power plants utilizing wet scrubbers with one sampling effort completed (report available 11/96). The fifth plant is yet to be identified. Completion of the Phase II sampling activities is planned for the second and third quarters of 1997, with the site reports available in 1998.

Key objectives of the HAP assessment program cover a broad range of technical issues. Some of these include the following:

- Generating HAP data for a variety of coal types, furnace types, and emission control systems in order to calculate emission factors for the 189 HAPs identified.

- Determining the effectiveness of commonly used emission control devices (electrostatic precipitators [ESPs], fabric filters [FF], spray dryer absorbers [SDAs], and wet scrubbers) to reduce HAP emissions.

- Determining the effectiveness of advanced emission control devices (advanced flue gas desulfurization [FGD] for sulfur dioxide $\left[\mathrm{SO}_{2}\right]$ reduction, and selective catalytic reduction [SCR] of nitrogen compounds $\left[\mathrm{NO}_{x}\right]$ ).

- Calculating subsystem and overall plant material balances for selected HAPs.

- Determining mercury speciation and related emission factors.

- Determining solid-phase HAP distribution as a function of particle size. 
- Determining particle-size distribution of stack emissions and ESP/FF hopper ash.

- Evaluating the performance of a stack-sampling method referred to as plumesimulating dilution sampling (PSDS).

- Evaluating chromium speciation and sampling methods.

- Evaluating the effect of sootblowing on trace element emissions.

- Determining the concentration of HAPs on particle surfaces.

- Determining the distribution of HAPs between solid, liquid, and vapor phases.

The eight Phase I sites represent a range of fuel characteristics, including bituminous, subbituminous, and lignite fuels. The geographic locations of the eight plants include the states of Georgia, Ohio (two sites), Illinois, Indiana, Minnesota, North Dakota, and Arizona. Furnace types included tangentially $(t)$-fired, opposed wall-fired, front wall-fired, and cyclone-fired units ranging in size from 75 to $615 \mathrm{MWe}$ gross.

Electrostatic precipitators were used to control particulate emissions on six of the nine system configurations, with reported particulate collection efficiencies of nominally $97 \%$ to $99.8 \%$, which represents marginal to highly efficient ESP control technology. Fabric filters were used to control particulate emissions on three system configurations: two reverse-gas units and one slipstream pulse-jet baghouse. Particulate collection efficiency ranged from nominally $99.8 \%$ to $99.98 \%$.

Sulfur dioxide control technologies were employed on five system configurations. The technologies represented included a lime-based spray dryer system, a conventional limestone wet FGD system, the Chiyoda Thoroughbred-121 FGD process, the Pure Air

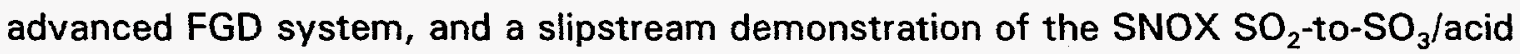
condensation process. Sulfur dioxide control ranged from $60 \%$ for the spray dryer system to $>90 \%$ for the wet-scrubbing concepts. The SNOX process demonstrated the highest level of sulfur dioxide control, $94 \%$ to $96 \%$. Two systems, $t$-fired units, used overfire air to reduce the emission of nitrogen species. However, $\mathrm{NO}_{x}$ reduction data were not reported. The SNOX slipstream demonstration system reported achieving $85 \%-93 \% \mathrm{NO}_{x}$ control using ammonia injection with an SCR catalyst.

\section{ES-4}


Sample collection for each of the nine system configurations varied somewhat from site to site because of site-specific characteristics. However, a thorough sampling approach was applied at each site to ensure sufficient solid-, liquid-, and gas-phase samples were collected from multiple locations to complete the HAP assessment. Solid samples included coal, ash, and FGD by-product/waste samples from dry and slurry/sluice streams. Liquid samples included water and slurry/sluice streams. Gas samples were generally limited to flue gas at the inlet and outlet of the particulate control device and in the stack and were reported on a dry gas basis and normalized to $3 \%$ oxygen.

The list of analytes for which sampling and analysis was specifically completed included trace elements, radionuclides, anions, inorganic compounds, and organic compounds. Several major elements were also included in order to permit a more thorough evaluation of mass balance results for trace elements. A variety of analytical methods were used to quantify the respective analytes. The sampling and analytical approach was developed to permit the quantitative determination of as many as possible of the 189 HAPs listed in the 1990 CAAA.

Quality assurance/quality control ( $Q A / Q C$ ) measures were a critical aspect of the overall program in order to ensure that meaningful data were obtained. Site-specific QA/QC plans were developed by each contractor to address sample collection, sample handling, sample analyses, data analyses, and specific corrective action to be taken when preestablished specifications were not met. In addition to the procedures established by the individual contractors for QA/OC audits, independent QA/QC audits funded by EPA were performed by Research Triangle Institute (RTI). QA/OC procedures included field blanks, spikes, documentation of detection limits, and a round-robin analyses of coal samples from each test site coordinated by CONSOL INC.

\section{RESULTS}

Although the results of the DOE Phase I air toxics study do not answer all of the questions concerning the emission of HAPs from coal-fired boilers, these data establish a good basis of information and will help to focus Phase II activities on the most 
pertinent questions. The technical areas addressed in this brief summary of results include coal analyses and emission factors for inorganic, organic, radionuclide, and acid gas-halogen elements and species. Coal analysis data are discussed in terms of individual plant data and round-robin results. Inorganic, organic, radionuclide, and acid gas-halogen data are primarily discussed in terms of stack concentration and emission factors. A limited discussion is also included concerning percent penetration data for trace elements and acid gases-halogens.

The basis of this discussion will be emission factors for the individual HAPs. Emission factors are emission estimates reported on a pound per trillion Btu (lb/10 $12 \mathrm{Btu})$ of heat input. The purpose for calculating emission factors was to provide a simple method for estimating annual HAP emissions on a ton per year basis. HAP emission control will be discussed in terms of percent penetration. Percent penetration values were calculated from the mean stack concentration (determined as a result of field measurements) divided by the theoretical stack concentration based on the assumption that $100 \%$ of the element or compound in the coal exited the stack. Penetration data were calculated for trace elements and acid gases-halogens for the purpose of evaluating the relative performance of emission control technologies.

\section{Round-Robin Coal Analyses}

Seventeen trace elements were included in the round-robin coal study: antimony $(S b)$, arsenic $(A s)$, barium $(B a)$, beryllium $(B e)$, boron $(B)$, cadmium $(C d)$, chromium $(C r)$, cobalt (Co), copper (Cu), lead ( $\mathrm{Pb})$, manganese $(\mathrm{Mn})$, mercury $(\mathrm{Hg})$, molybdenum (Mo), nickel (Ni), selenium (Se), vanadium (V), and fluorine (F). Other measurements determined in the round-robin study included proximate-ultimate analyses and major ash elements. The coal samples used in the round-robin study were supplied by the primary contractors at each of the test sites to CONSOL INC, which coordinated the study. Every feed coal used in Phase I was thus represented in the round-robin study. The five laboratories participating in the round-robin study were the same laboratories contracted to perform the field test analyses. 
National Institute of Standards and Technology (NIST) Standard Reference Material (SRM) 1632b, a Pittsburgh seam coal, was used to evaluate laboratory accuracy. Data within $10 \%$ of the NIST value were considered accurate. The accuracy of trace element analyses ranged from $38 \%$ to $75 \%$. The elements yielding the most problematic trace element data were $\mathrm{Sb}, \mathrm{As}, \mathrm{Cd}, \mathrm{Mo}$, and Se. No laboratory was able to report trace element content accurately more than $75 \%$ of the time. Overall, $57 \%$ of the trace element data reported for SRM $1632 b$ were accurate.

Interlaboratory reproducibility was evaluated using percent relative standard deviation (PRSD). The average PRSD for all coals and all contractors was $28 \%$. Average PRSD values for individual trace elements ranged from $11 \%$ for $V$ to $61 \%$ for Mo. The range of PRSDs was large: $\mathrm{Ba}, \mathrm{Cd}, \mathrm{Cu}, \mathrm{Hg}, \mathrm{Mo}, \mathrm{Ni}, \mathrm{Pb}$, and $\mathrm{Sb}$ all had PRSD ranges of over $30 \%$. For some samples, the range of reported values for $\mathrm{Mo}$, $\mathrm{Ni}$, and Cd was $52 \%, 76 \%$, and $110 \%$, respectively. These results indicate that outlier values are common in trace element analyses. Average PRSD correlated with coal heating value, indicating that as coal rank decreases, the analytical variability for trace elements increases.

Intralaboratory repeatability was calculated as the average percent difference in a single laboratory's results on eight duplicate samples. The average percent difference for trace elements was $15 \%$, ranging from a low of $7.8 \%$ for $\mathrm{Cr}$ to $33 \%$ for $\mathrm{Cd}$. Elements with low interlaboratory reproducibility also tended to have low intralaboratory repeatability.

Comparison of the round-robin results with the plant data showed major differences. In many cases, the plant results differed from the round-robin results by $25 \%$ or more for major elements, proximate-ultimate values, and trace element results. At times, these differences exceeded $100 \%$. Coal trace element content was observed to vary within about 1 order of magnitude for each element. These results are problematic, suggesting that the feed coal data used in mass balance and penetration calculations are a major source of uncertainty. 


\section{Trace Element Emission Factors}

Emission factor data were generated for 25 elements, including nine major elements and 16 minor or trace elements in the DOE Phase I study. The major elements included aluminum (Al), calcium ( $\mathrm{Ca})$, iron $(\mathrm{Fe})$, magnesium $(\mathrm{Mg})$, potassium $(\mathrm{K})$, sodium (Na), silicon (Si), strontium (Sr), and titanium (Ti). The primary purpose for including a number of major elements in the study was to permit a better assessment of material balance results. Minor or trace elements included $\mathrm{Sb}, \mathrm{As}, \mathrm{Ba}, \mathrm{Be}, \mathrm{B}, \mathrm{Cd}, \mathrm{Cr}, \mathrm{Co}, \mathrm{Cu}$, $\mathrm{Pb}, \mathrm{Mn}, \mathrm{Hg}, \mathrm{Mo}, \mathrm{Ni}$, Se, and $\mathrm{V}$.

Eleven of the trace elements ( $\mathrm{Sb}, \mathrm{As}, \mathrm{Be}, \mathrm{Cd}, \mathrm{Cr}, \mathrm{Co}, \mathrm{Pb}, \mathrm{Mn}, \mathrm{Hg}, \mathrm{Ni}$, and $\mathrm{Se}$ ) included in the DOE Phase I study are also found on the list of 189 HAPs identified in the CAAA of 1990.

A comparison of DOE Phase I stack concentration data with EPA ambient air data' collected since 1980 for 11 trace elements and four vapor-phase pollutants is presented in Figure ES-1. The data show that for nine ( $\mathrm{Sb}, \mathrm{As}, \mathrm{Be}, \mathrm{Cd}, \mathrm{Co}, \mathrm{Cr}, \mathrm{Hg}, \mathrm{Mn}$, and $\mathrm{Ni}$ ) of the 11 trace elements, the median stack concentrations are 2 to 3 orders of magnitude greater than the range of ambient air concentrations. ${ }^{1,2}$ For $\mathrm{Se}$ and $\mathrm{Pb}$, the differential was roughly 4 and 1 order of magnitude, respectively. The four vapor-phase species (hydrochloric acid $[\mathrm{HCl}]$, hydrogen fluoride [HF], $\mathrm{NO}_{x}$ and $\mathrm{SO}_{2}$ ) exhibit differentials ranging from 3 to 4 orders of magnitude. These data imply, with the exception of $\mathrm{Pb}$, that coal-fired power plants are possible contributors to ambient air concentrations for these trace elements. The level or degree of contribution can only be determined as a function of extensive dilution and dispersion modeling, which is beyond the scope of this review effort.

The variability of the DOE Phase I data demonstrates the difficulty involved in quantifying trace element emissions from coal-fired systems. The data in Figure ES-2 show that the emission factor range for a given element was as small as 1 order of

1. Kelly, T.J.; Ramamurthi, M.; Pollack, A.J.; Spicer, C.W. “Ambient Concentration Summaries for Clean Air Act Title III Hazardous Air Pollutants," final report for U.S. Environmental Protection Agency Contract No. 68-D80082; Battelle, July 1993.

2. Bureau of National Affairs. "100 Guide to the Law," In Air Pollution Control: BNA Policy and Practice Series; Bureau of National Affairs: Washington DC, 1994; pp 100:101-600. 


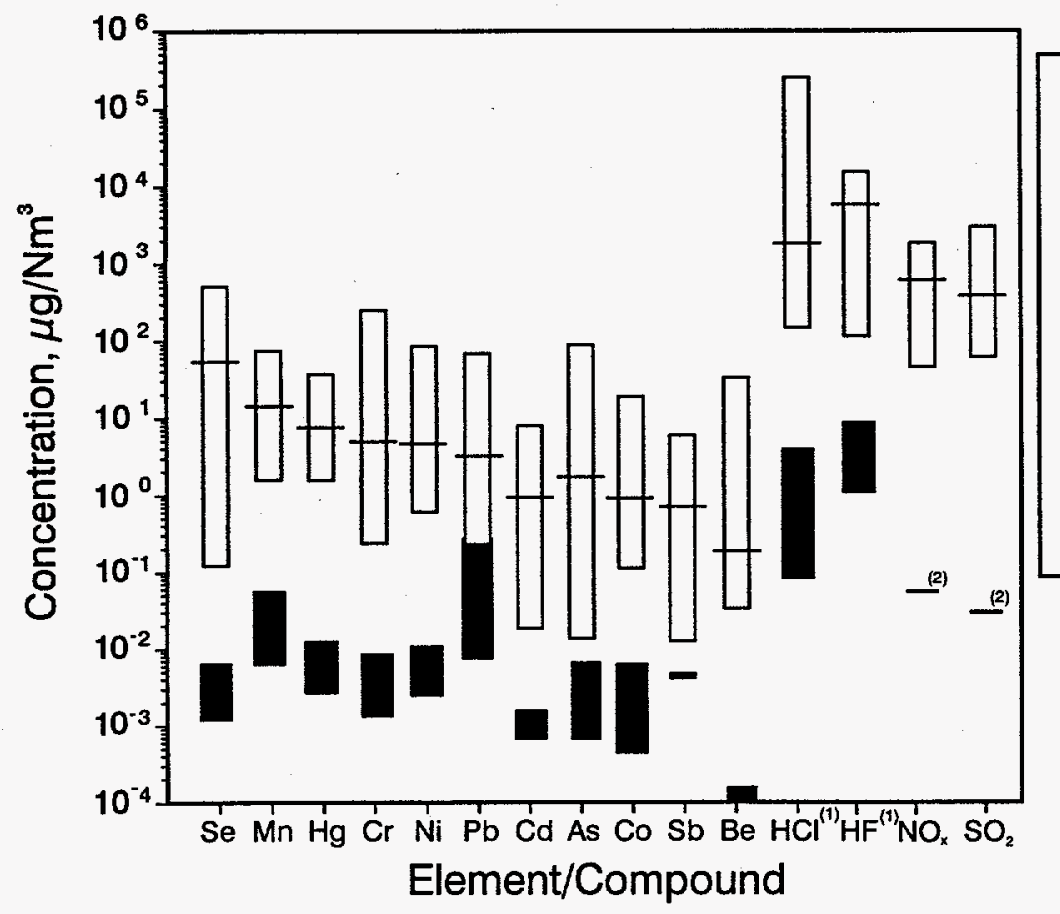

EERC SN12722A.CDR

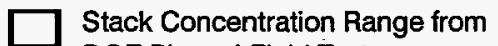
DOE Phase I Field Tests

Ambient Air Concentration Range from Rural and Urban Areas Since 1980

Median Concentration

Notes: Plant data median value calculation uses nondetect values equal to detection limits.

(1) Plant data are combined $\mathrm{HCl}+\mathrm{Cl}_{2}$ and $\mathrm{HF}+\mathrm{F}_{2}$. Ambient data are assumed to be combined also. Median not available for ambient air levels.

(2) Ambient air quality standard.

Figure ES-1. DOE Phase I Stack Concentration Data for 11 Trace Elements and Four Acid Gases Compared to EPA Ambient Air Data and Quality Standards.

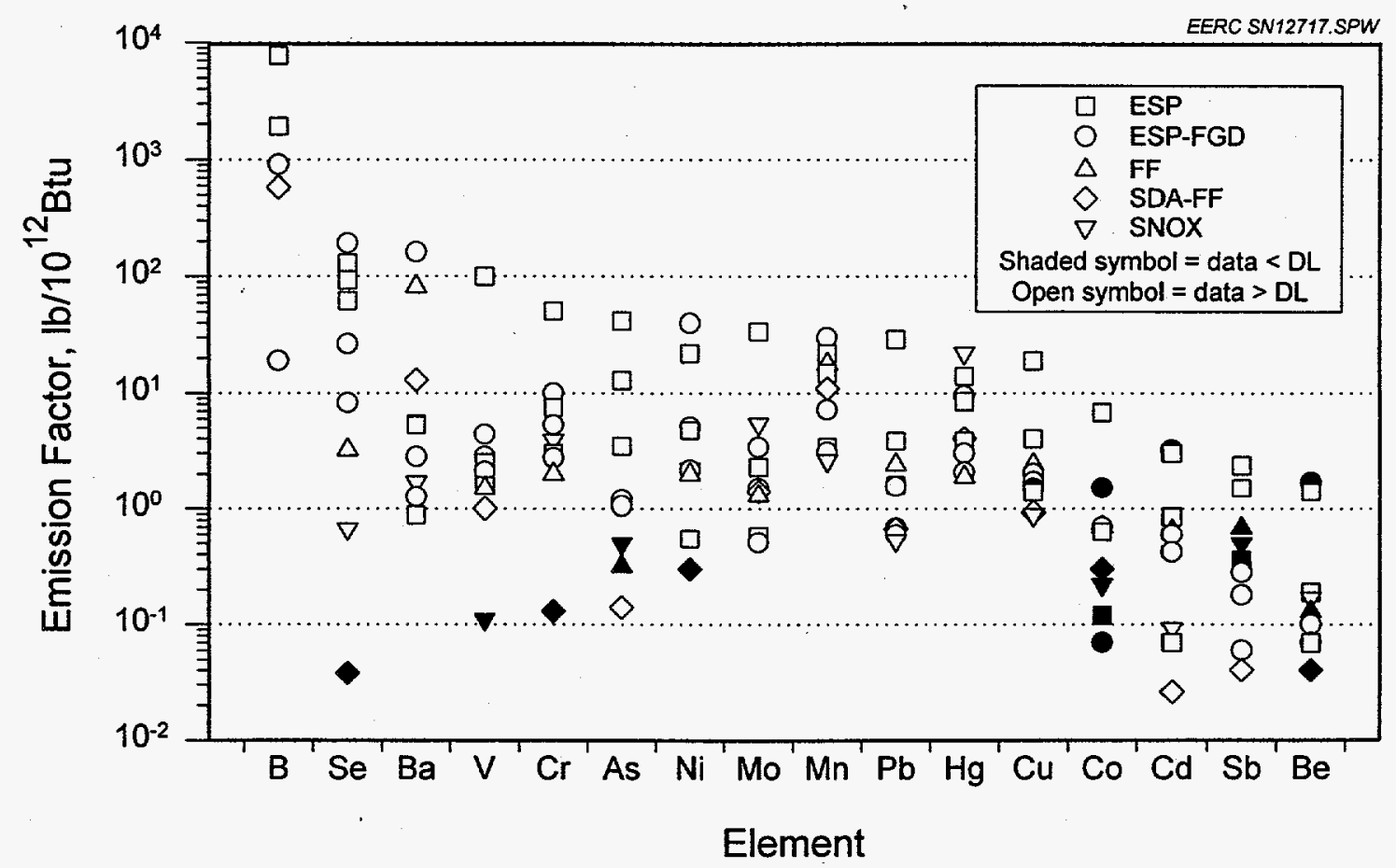

Figure ES-2. Emission Factors for 16 Trace Elements at Each of Nine DOE Phase I Field Process Configurations. 
magnitude for $\mathrm{Mn}\left(2.6\right.$ to $\left.30 \mathrm{lb} / 10^{12} \mathrm{Btu}\right)$ and $\mathrm{Hg}\left(1.9\right.$ to $\left.22 \mathrm{lb} / 10^{12} \mathrm{Btu}\right)$ to nearly 4 orders of magnitude for Se (ND [nondetect] $<0.038$ to $193 \mathrm{lb} / 10^{12} \mathrm{Btu}$ ). The trace element listed as a HAP having the highest median emission factor was Se $\left(26.5 \mathrm{lb} / 10^{12} \mathrm{Btu}\right)$, followed closely by $\mathrm{Mn}\left(11 \mathrm{lb} / 10^{12} \mathrm{Btu}\right)$. Sb and Be were observed to have the lowest median emission factors, ND $<0.36$ and ND $<0.13 \mathrm{lb} / 10^{12} \mathrm{Btu}$, respectively.

Based on the Phase I Se data, it is not possible to clearly delineate the potential to control Se emissions from coal-fired utility boilers using existing emission control technologies. Also, the relative value of the Se data for developing conclusions for a large population of coal-fired boilers is limited because of the small size of the data set, the large number of variables represented ffuel types, boiler types, emission control systems, etc.), and the variability of some of the data. In the DOE Phase I field sampling effort, $>90 \%$ Se control was observed only for sites employing either a fabric filter or a combination of particulate and acid gas control technology. For the SNOX process, $>99 \%$ of the Se was in the sulfuric acid (usable by-product).

Emission factors for total $\mathrm{Hg}$ ranged from 1.9 to $22 \mathrm{lb} / 10^{12} \mathrm{Btu}$, based on mean stack concentrations of 2.6 to $30 \mu \mathrm{g} / \mathrm{Nm}^{3}\left(\mathrm{Nm}^{3}\right.$ is based on $0^{\circ} \mathrm{C}$ and 1 atmosphere). Stack concentrations were reported on a dry basis normalized to $3 \%$ oxygen. Emission factor and stack concentration data correlated somewhat with the $\mathrm{Hg}$ concentration in the coal (mean values of 0.04 to $0.28 \mu \mathrm{g} / \mathrm{g}$ ). Typical mean $\mathrm{Hg}$ values of about $0.1 \mu \mathrm{g} / \mathrm{g}$ were reported for six of the eight fuels. Calculated percent penetration values for total $\mathrm{Hg}$ ranged from about $25 \%$ to nearly $120 \%$. From the DOE Phase I data, the potential to control $\mathrm{Hg}$ emissions from coal-fired utility boilers using existing emission control technologies is unclear. It is also important to remember that the control of volatile or vapor-phase HAPs is not likely to exceed control levels observed for vapor-phase priority pollutants such as $\mathrm{SO}_{2}$. Therefore, if $\mathrm{Hg}$ regulations are promulgated for coalfired utility systems, existing control technologies will require augmentation, and alternative control technology options will require development. Also, evaluating emission control technology performance based on total $\mathrm{Hg}$ concentration alone is not appropriate, since $\mathrm{Hg}$ speciation may affect the degree of control observed. This effect currently cannot be quantified adequately, since methods to speciate $\mathrm{Hg}$ are still 
unproven. Based on the DOE Phase I Hg data, future $\mathrm{Hg}$-sampling efforts must emphasize accurate $\mathrm{Hg}$ speciation in order to evaluate the performance of emission control technologies properly.

The data for the nonvolatile trace element $(\mathrm{Sb}, \mathrm{As}, \mathrm{Ba}, \mathrm{Be}, \mathrm{Cd}, \mathrm{Cr}, \mathrm{Co}, \mathrm{Cu}, \mathrm{Pb}$, $\mathrm{Mn}, \mathrm{Mo}, \mathrm{Ni}$, and $\mathrm{V}$ ) emission factors indicate that emission control for these trace elements is directly related to overall particulate control for the individual field sites. Emission control for the 13 nonvolatile trace elements was $>90 \%$ for all nine process configurations. Particulate control alone (ESP or FF) limited penetration to $<5 \%$ (>95\% control) for ten ( $\mathrm{Sb}, \mathrm{Ba}, \mathrm{Be}, \mathrm{Cr}, \mathrm{Co}, \mathrm{Cu}, \mathrm{Pb}, \mathrm{Mn}, \mathrm{Ni}$, and $\mathrm{V}$ ) of the 13 trace elements. A combination of particulate control and dry or wet FGD demonstrated $>99 \%$ control for eight ( $\mathrm{Sb}, \mathrm{As}, \mathrm{Ba}, \mathrm{Co}, \mathrm{Cu}, \mathrm{Pb}, \mathrm{Mn}$, and $\mathrm{V}$ ) of the 13 trace elements. The exceptions noted appear to be the result of high reported nondetect concentrations, failed blanks and/or spike recoveries, and significant data variability. Therefore, the DOE Phase I data indicate that the emission of the 13 nonvolatile trace elements was effectively controlled ( $>98 \%$ ) by highly efficient particulate control technology or technology combinations (ESP-FGD or SDA-FF) currently being used by the U.S. utility industry. However, the control of trace element emissions will never exceed the level of overall particulate control observed.

\section{Organic Emission Factors}

Four types of organic compound classes on the EPA list of 189 HAPs were sampled at nine utility field process configurations. The four organic compound classes are 1) aldehydes and ketones, 2) volatile organic compounds (VOCs), 3) semivolatile organic compounds (SVOCs) and polycyclic aromatic hydrocarbons (PAHs), and 4) chlorinated dioxins and furans.

Although organic emission factors from data obtained from the nine utility field process configurations are reported, the quality of organic results varied from contractor to contractor, and the overall results were quite variable. Major problems associated with the organic results included 1) low concentrations of the organic analytes found in the stacks of these sites, requiring a majority of the analyses to be performed at or very 
near their detection limits; 2) inadequate methods for aldehydes and ketones; 3) high blank values and poor spike and surrogate recoveries; and 4/ nonuniform administration of external spiking audits (e.g., poor recoveries of spiked audit samples as reported by a few contractors, without any apparent corrective actions taken).

The organic emission factors summarized in this report by the EERC take into consideration the results from blank samples, spiked, and audit samples and detection limits for individual organic compounds. Since there were significant problems in organic analyses, the organic emission factors provided in this summary report should be considered only as a representative upper limit range of potential organic emissions from coal-fired power plants and should not be used for any quantitative projection of emissions. While some $\mathrm{QA} / \mathrm{OC}$ problems were evident, the organic results indicated that the overall concentrations of organic pollutants released from coal-fired utility power plants are low (comparable to ambient concentrations for some species), as evidenced by the generally low organic emission factors presented in this summary report.

Results from only three of the nine field process configurations were included in the emission factor table for aldehydes and ketones. Data from these three sites indicated that stack emissions of aldehydes are quite low, ranging between $<2$ and $41 \mathrm{lb} / 10^{12}$ Btu for acrolein and between 67 and $390 \mathrm{lb} / 10^{12}$ Btu for acetaldehyde.

VOC species detected in the stack emissions were quite low in concentration. In general, quantitative VOC results were suspect, mainly because concentrations reported were often very near detection limits. Only benzene and chloromethane stack concentrations were found to exceed the EPA median ambient air concentrations. ${ }^{3}$ Emission factors for benzene and chloromethane ranged between 1 and $120 \mathrm{lb} / 10^{12}$ Btu and between 5 and $218 \mathrm{lb} / 10^{12}$ Btu, respectively.

The SVOC and PAH species were also found in quite low concentrations, often near the detection limit. The concentration of the SVOC and PAH compound classes EPA/600/3-88/010(a); U.S. Environmental Protection Agency Contract No. 68-02-4190, Feb. 1988.

\section{ES-12}


found ranged from 0.4 to $9 \mathrm{lb} / 10^{12} \mathrm{Btu}$ for phenol and from 0.0021 to 0.005 $\mathrm{lb} / 10^{12}$ Btu for benz[a]anthracene. Species included in EPA's National Ambient VOC database that were reported for some of the power stations included phenol, methylphenols, naphthalene, and methylnaphthalene. These species were present in the stacks at concentrations similar to ambient concentrations.

Chlorinated dioxins and furans were found at much lower concentrations than other compound classes (e.g., SVOC and PAH), typically 6 orders of magnitude lower. Emission factors were typically $<3 \times 10^{-6}$ to $<6 \times 10^{-6} \mathrm{lb} / 10^{12} \mathrm{Btu}$. The very low levels of chlorinated dioxins and furans found in the stack indicated that chlorinated dioxin and furan emissions are not significant from the coal-fired utility sites included in the DOE Phase I study.

\section{Radionuclide Emission Factors}

Radionuclides are listed generically as a CAAA HAP. All of the contractors provided radionuclide data, but the selection of radionuclides for which results were reported varied greatly among contractors. Results for Pb-210 (lead), Ra-226 (radium), and U-235 (uranium) were reported for all field sites. Results for Ra-228, Th-230 (thorium), and U-234 were reported for all sites except one. Results for the remaining radionuclides (Pb-211, Pb-212, Po-210 [polonium], Th-228, Th-229, Th-232, Th-234, and $U-238$ ) were reported for three to five sites each. Some contractors reported radionuclide results for feed coal only but most contractors listed results for additional solid samples. Radionuclide data for feed coal and stack gas samples as well as emission factors and control device efficiencies are presented in Section 4.4 of this summary report.

Most of the contractors reported a variety of radionuclides found in the plant feed coals; only Pb-211 and Th-229 were not detected in these samples. The range of detectable radionuclide values in the feed coal samples was 0.02 to $7.3 \mathrm{pCi} / \mathrm{g}$. Radionuclides were reported with much less frequency in the stack gas samples. $\mathrm{Pb}-211, \mathrm{~Pb}-212, \mathrm{Ra}-228$, Th-228, Th-229, Th-232, and Th-234 were not reported in 
stack gas samples at any of the sites. Pb-210, Po-210, Ra-226, Th-230, U-234, and U-235 were reported at one site each. U-238 was reported at two sites.

Average emission factors on a mass basis for the reported radionuclides ranged from $3.9 \times 10^{-10} \mathrm{lb} / 10^{12}$ Btu for Po-210 to $312 \mathrm{lb} / 10^{12} \mathrm{Btu}$ for U-238. Most of the other radionuclides had average emission factors on a mass basis in the range of $10^{-7}$ to $10^{-4} \mathrm{lb} / 10^{12}$ Btu. Average emission factors on an activity basis for the reported radionuclides ranged from $1.4 \times 10^{8} \mathrm{pCi} / 10^{12} \mathrm{Btu}$ for $\mathrm{Ra}-226$ to $7.2 \times 10^{10} \mathrm{pCi} / 10^{12} \mathrm{Btu}$ for $\mathrm{Pb}-210$. Most of the average emission factors on an activity basis were in the range of $10^{8}$ to $10^{9} \mathrm{pCi} / 10^{12} \mathrm{Btu}$.

\section{Acid Gas-Halogen Emission Factors}

Total chlorine $\left(\mathrm{HCl}+\mathrm{Cl}_{2}\right)$ emission factors were, in general, 1 to 2 orders of magnitude larger for ESP systems than for scrubber and FF systems. Total chlorine emissions ranged from ND $<176$ to $132,000 \mathrm{lb} / 10^{12}$ Btu. Estimated annual emission rates were 1.6 to 1645 tons/yr. Total fluorine $\left(\mathrm{HF}+\mathrm{F}_{2}\right.$ ) emissions ranged from ND $<92$ to $12,770 \mathrm{lb} / 10^{12} \mathrm{Btu}$. Emission factors for hydrogen cyanide (HCN) ranged from ND $<2.2$ to $180 \mathrm{lb} / 10^{12}$ Btu.

Total fluorine emission factors followed the same trends as total chlorine, except the Boswell Energy Center (FF) showed emission factors similar to ESP-only systems. Niles, Baldwin, and Cardinal Stations (all ESPs) had emission factors of 8921, 9900, and $12,770 \mathrm{lb} / 10^{12} \mathrm{Btu}$, respectively. Niles/SNOX Station (FF with acid condenser), Boswell Energy Center (FF), and Coal Creek Station (ESP-wet FGD with $40 \%$ bypass) had emission factors of 6630,3310 , and $3980 \mathrm{lb} / 10^{12}$ Btu, respectively. Emissions from scrubber systems were very low or in the nondetect range. Where QA/OC was performed for anion data, it was satisfactory; however, the averaged data show large standard deviations.

While $\mathrm{HCl}, \mathrm{Cl}_{2}$, and $\mathrm{HF}$ are on the list of $189 \mathrm{HAPs}, \mathrm{F}_{2}$ is not. For the three stations which speciated acid gases, $F_{2}$ represented $0 \%, 25 \%$, and $85 \%$ of total fluorine. Chlorine gas $\left(\mathrm{Cl}_{2}\right)$ represented $5.5 \%, 45 \%$, and $6.3 \%$ of total chlorine, 
respectively. Given the wide variability of this limited data set, it is not possible to predict the ratio of $\mathrm{F}_{2} / \mathrm{HF}$ or $\mathrm{Cl}_{2} / \mathrm{HCl}$ for a given system. Bromine gas $\left(\mathrm{Br}_{2}\right)$ and hydrogen bromide $(\mathrm{HBr})$ were not detected at the two sites where analyses were performed.

As expected, removal of $\mathrm{HCl}, \mathrm{HF}$, and $\mathrm{HCN}$ did not occur across ESPs. The FF at Boswell Energy Center did show acid gas-halogen removals, possibly because of reactions or adsorption in the filter dust cake, of $35 \%-65 \% \mathrm{HCl}$, (negative) $\mathrm{HF}$, $60 \%-97 \% \mathrm{Cl}_{2}, 53 \%-72 \% \mathrm{~F}_{2}$, and $35 \% \mathrm{HCN}$. However, no overall acid gas removal was shown for the FF at the Niles/SNOX Station.

Also, as expected, removal of $\mathrm{HCl}, \mathrm{HF}$, and $\mathrm{HCN}$ were significant across wet $\mathrm{FGD}$ systems, $>99 \% \mathrm{HCl},>96 \% \mathrm{HF}$, and $\mathrm{HCN}$ to a lesser extent. The jet bubbling reactor (JBR) at Plant Yates efficiently removed (>99\%) of high inlet anion concentrations. Total acid gas removal for the Coal Creek Station wet FGD system was diminished by half because of the $40 \%$ bypass, and sampling leaks at Springerville precluded any conclusions for the SDA.

Particulate-phase chloride $\left(\mathrm{Cl}^{-}\right)$, fluoride $\left(\mathrm{F}^{-}\right)$, and cyanide $\left(\mathrm{CN}^{-}\right)$were measured below detection limits for most of the sites and contributed $<5 \%$ of the total concentration for the remaining sites. Fabric filter and ESP removal for $\mathrm{Cl}^{-}$was $>90 \%$ to $99 \%$ for all sites, whereas $F^{-}$showed greater variability, with $55 \%-99 \%$ removal. Cyanide, where measured above the detection limit, showed removal efficiency around $35 \%$.

\section{Estimates of Total HAP Emissions}

Table ES- 1 summarizes DOE Phase I emission factor data on a lb/10 12 Btu basis for $\mathrm{HAP}$ trace elements, organic compounds, radionuclides, $\mathrm{Cl}_{2}, \mathrm{HCl}, \mathrm{HF}$, and $\mathrm{HCN}$. Trace element emission factors include a number of values indicated as nondetect values. These nondetect values were included in the emission factor totals assuming a worst-case scenario. As a result, total HAP trace element emission factors ranged from 17 to $284 \mathrm{lb} / 10^{12} \mathrm{Btu}$. Based on fuel feed rates, fuel analyses reported for the nine process configurations, and an overall average capacity factor of 0.7 , the total annual 
Table ES-1. A Summary of DOE Phase I Hazardous Air Pollutant Emission Factor Data

\begin{tabular}{|c|c|c|c|c|c|c|c|c|c|}
\hline Plant Name: & Bailly & Baldwin & Boswell & Cardinal & Coal Creek & Niles & SNOX & Springerville & Yates \\
\hline \multicolumn{10}{|c|}{ Trace Elements, $\mathrm{lb} / 10^{12} \mathrm{Btu}$} \\
\hline Sb & 0.28 & 1.5 & $0.68^{\mathrm{a}}$ & 2.4 & 0.18 & $0.36^{\mathrm{a}}$ & $0.5^{a}$ & 0.04 & 0.06 \\
\hline As & 1.1 & 13 & $0.32^{\mathrm{a}}$ & 3.5 & 1.2 & 42 & $0.5^{\mathrm{a}}$ & 0.14 & 1.2 \\
\hline $\mathrm{Be}$ & $0.07^{a}$ & 1.4 & $0.13^{\mathrm{a}}$ & 0.07 & $1.7^{\mathrm{a}}$ & 0.19 & 0.17 & $0.04^{\mathrm{a}}$ & 0.1 \\
\hline Cd & 0.42 & 3 & $0.65^{a}$ & 0.85 & $3.2^{\mathrm{a}}$ & 0.07 & $0.09^{a}$ & 0.03 & 0.6 \\
\hline $\mathrm{Cr}$ & 2.7 & 51 & 2 & 7.5 & 10 & 3 & 3.9 & $0.13^{a}$ & 5.3 \\
\hline Co & $0.07^{a}$ & 6.8 & 0.7 & 0.63 & $1.5^{\mathrm{a}}$ & $0.12^{\mathrm{a}}$ & $0.22^{a}$ & $0.3^{\mathrm{a}}$ & 0.7 \\
\hline $\mathrm{Pb}$ & 1.6 & 29 & 2.4 & 3.8 & 0.69 & 1.6 & 0.53 & 0.67 & 0.6 \\
\hline Mn & 3.1 & 22 & 18 & 20 & 30 & 3.4 & 2.6 & 11 & 7.2 \\
\hline $\mathrm{Hg}$ & 2.1 & 3.8 & 1.9 & 8.5 & 9.5 & 14 & 22 & 4 & 3 \\
\hline $\mathrm{Ni}$ & 2.2 & 22 & 2 & 4.8 & 5.1 & 0.55 & 2.2 & $0.3^{\mathrm{a}}$ & 40.1 \\
\hline $\mathrm{Se}$ & 193 & 130 & 3.3 & 93 & 8.3 & 62 & 0.67 & $0.02^{a}$ & 26.5 \\
\hline Total Trace Elements & 206.64 & 283.5 & 32.08 & 145.05 & 71.37 & 127.29 & 33.38 & 16.67 & 85.36 \\
\hline \multicolumn{10}{|l|}{ Organics, lb/10'2 Btu } \\
\hline Aldehydes & $N A_{b}^{b}$ & $N A^{b}$ & $N A^{b}$ & $N A^{b}$ & 79 & 159 & 468 & $N A^{b}$ & $N A^{b}$ \\
\hline VOCs & $N A_{b}^{b}$ & 147 & 250 & 117 & 219 & 44 & 262 & 2 & 5 \\
\hline SVOCs & $N A_{b}^{b}$ & 35 & 4.3 & $\mathrm{NA}^{\mathrm{b}}$ & 1.6 & 2.3 & 0.6 & $N A^{b}$ & 141 \\
\hline Total Organics & $N A^{b}$ & 182 & 254.3 & 117 & 299.6 & 205.3 & 730.6 & 2 & 146 \\
\hline $\begin{array}{c}\text { Radionuclides, } \\
\text { lb } / 10^{12} \mathrm{Btu}\end{array}$ & $\mathrm{NA}^{\mathrm{b}}$ & 4 & $1^{a}$ & $628^{\circ}$ & $14^{a}$ & $41^{a}$ & 47 & $N A^{b}$ & $\mathrm{NA}^{\mathrm{b}}$ \\
\hline $\begin{array}{l}\text { Chlorine, } \mathrm{Ib} / 10^{12} \mathrm{Btu} \\
\mathrm{HCl}, \mathrm{lb} / 1 \mathrm{O}^{12} \mathrm{Btu}\end{array}$ & $\begin{array}{l}\text { NA } \\
1020\end{array}$ & $\begin{array}{l}4500 \\
78000\end{array}$ & $\begin{array}{l}640 \\
790\end{array}$ & $\begin{array}{l}1550 \\
22900\end{array}$ & $\begin{array}{r}\mathrm{NA}^{\mathrm{e}} \\
1340\end{array}$ & $\begin{array}{l}N^{e} \\
132000\end{array}$ & $\begin{array}{l}N A^{\ominus} \\
82400\end{array}$ & $\mathrm{NA}^{\mathrm{e}}$ & $\begin{array}{l}\text { NA } \\
531\end{array}$ \\
\hline $\begin{array}{l}\mathrm{HF},{ }^{9} \mathrm{Ib} / 10^{12} \mathrm{Btu} \\
\text { Cyanide, } \mathrm{Ib} / 10^{12} \mathrm{Btc}\end{array}$ & $\begin{array}{l}420^{a} \\
17.5\end{array}$ & $\begin{array}{l}9900 \\
2.2^{\mathrm{a}}\end{array}$ & $\begin{array}{r}2500 \\
3.7\end{array}$ & $\begin{array}{r}1870 \\
0.6\end{array}$ & $\begin{array}{r}3980 \\
51\end{array}$ & $\begin{array}{r}8921 \\
180\end{array}$ & $\begin{array}{r}6630 \\
157\end{array}$ & $\begin{array}{l}92^{a} \\
11^{a}\end{array}$ & $\begin{array}{r}122 \\
28\end{array}$ \\
\hline $\begin{array}{l}\text { Total HAPs, lb/10 } 12 \text { Btu } \\
\text { Coal Feed Rate, ton } / \mathrm{yr} \\
\text { Heating Value, Btu/lb } \\
\text { Cap. Factor }\end{array}$ & $\begin{array}{c}1658.14 \\
2010516 \\
11100 \\
0.7\end{array}$ & $\begin{array}{c}92871.7 \\
2687373 \\
10600 \\
0.7\end{array}$ & $\begin{array}{l}4221.08 \\
322253 \\
8800 \\
\quad 0.7\end{array}$ & $\begin{array}{c}27210.6 \\
1865784 \\
12200 \\
0.7\end{array}$ & $\begin{array}{c}5755.97 \\
4922233 \\
6230 \\
0.7\end{array}$ & $\begin{array}{c}141474.6 \\
407997 \\
12175 \\
\quad 0.7\end{array}$ & $\begin{array}{l}89997.98 \\
407997 \\
12175 \\
\quad 0.7\end{array}$ & $\begin{array}{c}297.67 \\
1833986 \\
9450 \\
0.7\end{array}$ & $\begin{array}{c}912.36 \\
393470 \\
11200 \\
0.7\end{array}$ \\
\hline $\begin{array}{l}\text { Trace Element, ton/yr } \\
\text { Organics, ton/yr } \\
\mathrm{HCl}+\mathrm{Cl}_{2} \text { ton/yr } \\
\mathrm{HF} \text {, ton } / \mathrm{yr} \\
\text { Other, ton } / \mathrm{yr} \\
\mathrm{HAPs} \text {, ton } / \mathrm{yr}\end{array}$ & $\begin{array}{r}3.23 \\
0.00 \\
15.93 \\
6.56 \\
0.18 \\
25.90 \\
\end{array}$ & $\begin{array}{r}5.65 \\
3.63 \\
1645.08 \\
197.41 \\
0.12 \\
1851.89 \\
\end{array}$ & $\begin{array}{l}0.06 \\
0.50 \\
2.84 \\
4.96 \\
0.01 \\
8.38 \\
\end{array}$ & $\begin{array}{r}2.31 \\
1.86 \\
389.58 \\
29.80 \\
10.02 \\
433.57 \\
\end{array}$ & $\begin{array}{r}1.53 \\
6.43 \\
28.76 \\
85.43 \\
1.40 \\
123.56 \\
\end{array}$ & $\begin{array}{r}0.44 \\
0.71 \\
458.98 \\
31.02 \\
0.77 \\
491.93 \\
\end{array}$ & $\begin{array}{r}0.12 \\
2.54 \\
286.52 \\
23.05 \\
0.71 \\
312.94 \\
\end{array}$ & $\begin{array}{l}0.20 \\
0.02 \\
2.14 \\
1.12 \\
0.13 \\
3.61 \\
\end{array}$ & $\begin{array}{l}0.26 \\
0.45 \\
1.64 \\
0.38 \\
0.09 \\
2.81 \\
\end{array}$ \\
\hline $\begin{array}{l}\text { Based on data reporte } \\
\text { bata were highly ques } \\
\text { C Radionuclide data in tt } \\
\text { are presented on a me } \\
\text { Value appears to be a } \\
\text { Chlorine data not avai } \\
\mathrm{HCl} \text { measurement rep } \\
\mathrm{HF} \text { data represent con } \\
\text { and HF. }\end{array}$ & $\begin{array}{l}\text { ess than det } \\
\text { onable basec } \\
\text { table are pr } \\
\text { and activit } \\
\text { malous, but } \\
\text { le because } \\
\text { sents combir } \\
\text { ned } F_{2} / \mathrm{HF} \mathrm{e}\end{array}$ & $\begin{array}{l}\text { ection limits } \\
\text { on OA/QC } \\
\text { esented on a } \\
\text { basis in Se } \\
\text { no discussio } \\
\text { if combined } \\
\text { ed } \mathrm{Cl}_{2} / \mathrm{HCl}\end{array}$ & $\begin{array}{l}\text { criteria. } \\
\text { mass basis } \\
\text { ction } 4.4 \text { of } \\
\mathrm{Cl}_{2} / \mathrm{HCl} \text { mea }\end{array}$ & $\begin{array}{l}\text { order to } \\
\text { is report. } \\
\text { the cont } \\
\text { rement. } \\
\text { hlorine da }\end{array}$ & $\begin{array}{l}\text { tablish HAP } \\
\text { ctors' report }\end{array}$ & emissions & . & sis. Radionucli & lide data \\
\hline
\end{tabular}

emission rates at each plant for individual trace element $(\mathrm{Sb}, \mathrm{As}, \mathrm{Be}, \mathrm{Cd}, \mathrm{Cr}, \mathrm{Co}, \mathrm{Pb}$, $\mathrm{Mn}, \mathrm{Hg}, \mathrm{Ni}$, and $\mathrm{Se}$ ) HAPs were all estimated to be $<3$ tons/yr, and most were substantially, $<0.5$ tons/yr. The estimated combined trace element HAP annual emission rates for each of the nine process configurations ranged from 0.06 to 5.65 tons/yr.

Organic HAP data in Table ES-1 also indicate a very low emission rate for coalfired systems. For some plants, very little organic data are reported. This occurred as a result of the low concentrations encountered and questionable data quality. The 
approach for the reporting of organic data in this report was different than the approach taken for trace elements because, based on fuel analysis, the trace elements are known to be present. Fairly complete organic data are reported here for five of the nine Phase I process configurations. Total organic emission factors for these sites ranged from 182 to $731 \mathrm{lb} / 10^{12}$ Btu. The estimated organic HAP annual emission rates for these same five sites ranged from 0.5 to 6.4 tons/yr.

Radionuclide and cyanide emissions were also quite low, ranging from ND $<1$ to $47 \mathrm{lb} / 10^{12} \mathrm{Btu}$ and ND $<2.2$ to $180 \mathrm{lb} / 10^{12} \mathrm{Btu}$, respectively. The one high radionuclide value reported, $628 \mathrm{lb} / 10^{12} \mathrm{Btu}$, is believed to be anomalous. However, the contractor report does not specifically discuss this possibility. Estimated annual emission rates for this combination of HAPs were 0.01 to 1.4 tons/yr.

The data in Table ES-1 show that $\mathrm{Cl}_{2}, \mathrm{HCl}$, and $\mathrm{HF}$ were found in significant quantities at six of the nine DOE Phase I process configurations. Chlorine gas emissions were measured at only three sites, with emission factors ranging from 640 to $4500 \mathrm{lb} / 10^{12} \mathrm{Btu}$. For the other six sites, total chlorine emissions ranged from ND $<176$ to $132,000 \mathrm{lb} / 10^{12} \mathrm{Btu}$. These emission factors result in estimated annual emission rates of 1.6 to 1645 tons/yr. Hydrogen fluoride emission factors ranged from $\mathrm{ND}<92$ to $9900 \mathrm{lb} / 10^{12} \mathrm{Btu}$. These data show that the emissions of $\mathrm{Cl}_{2}, \mathrm{HCl}$, and $\mathrm{HF}$ depend on the $\mathrm{Cl}$ and $\mathrm{F}$ content of the fuel and that these emissions can be effectively controlled using conventional dry and wet scrubbing technologies.

Figure ES-3 summarizes estimated total annual HAP emissions for the nine DOE Phase I process configurations in a series of pie charts. This figure graphically depicts the small contribution made by the trace elements, organics, and other (radionuclide and cyanide) compounds to the total annual estimated emissions for each site. Therefore, it would appear that the emission of HAPs from coal-fired systems is not significant as long as the emission of $\mathrm{Cl}_{2}, \mathrm{HCl}$, and $\mathrm{HF}$ are effectively controlled.

The annual HAP emission values presented in Table ES-1 and Figure ES-3 are general estimates that can change significantly as a result of changing fuels, fuel characteristics, and fuel feed rates for an individual plant. Also, this discussion was based on an overall average capacity factor of 0.7 . The actual capacity factor for a 

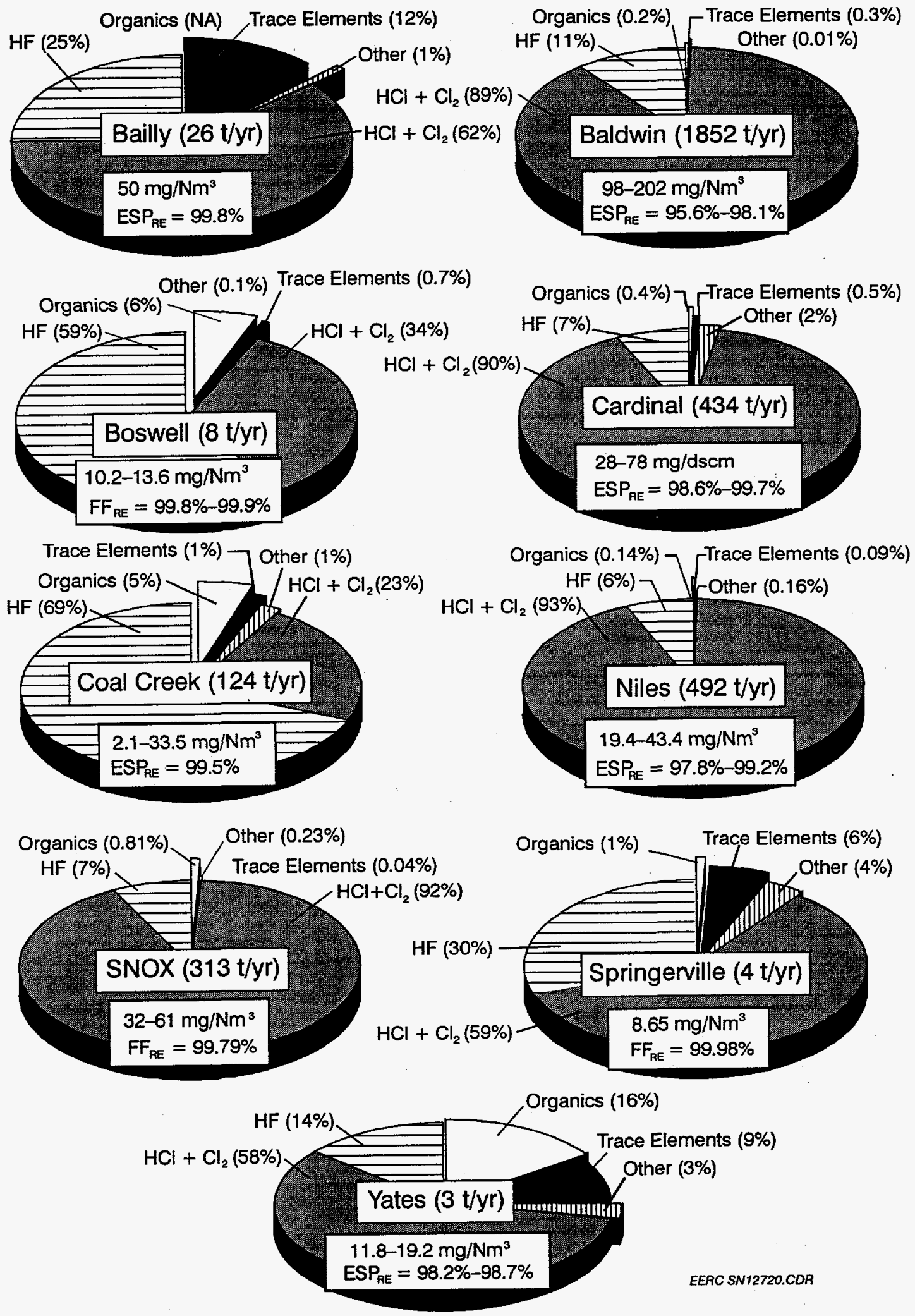

Figure ES-3. Summary of Estimated Annual HAP Emissions for the Nine DOE Phase I Process Configurations Including Stack Particulate Mass Loading $\left(\mathrm{mg} / \mathrm{Nm}^{3}\right.$ ) and Particulate Removal Efficiency (RE). 
given plant may be significantly different, depending on whether a plant is loadfollowing or typically base-loaded. Also, unplanned maintenance outages in any given year can greatly affect the capacity factor. Increasing the capacity factor from 0.7 to 0.85 would increase the estimated HAP emissions in Table ES-1 by about $20 \%$. Also, reducing the capacity factor from 0.7 to 0.5 would reduce the estimated HAP emissions by nearly $30 \%$.

The CAAA of 1990 will reduce and ultimately cap $\mathrm{SO}_{2}$ emissions from coal-fired electrical generating facilities by the year 2000. As a result, the installation of additional FGD capacity to meet Title IV, Acid Deposition Control, requirements will also significantly reduce $\mathrm{HAP}$ emissions of trace elements, $\mathrm{Cl}_{2}, \mathrm{HCl}, \mathrm{HF}$, and $\mathrm{HCN}$. 


\subsection{INTRODUCTION}

The University of North Dakota Energy \& Environmental Research Center (EERC), at the request of the U.S. Department of Energy Pittsburgh Energy Technology Center (DOE PETC), reviewed reports documenting the results from sampling activities completed at eight utility field sites. The purpose of the field sampling activities was to document the types and concentrations of potentially hazardous air pollutants (HAPs) from a select group of utility stations representing a cross section of U.S. coal-fired utility boilers. The overall review effort was conducted under DOE-EERC Cooperative Agreement No. DE-FC21-93MC30097 as Subtask 2.3 - Review and Assessment of Results from the Comprehensive Characterization of Toxic Emissions from Coal-Fired Power Plants.

This document represents the final report for Subtask 2.3.3 of the overall review effort. The objective was to provide a concise overview of the important findings from the Phase I air toxics assessment. Information is presented concerning the eight field sites at which sampling was completed assessing nine system configurations. Technical information is presented concerning the round-robin coal analyses and individual site data; the emission factors for inorganic, organic, radionuclide, and acid gas-halogen species; the effects of coal characteristics and process configurations on emission factors; and several special topics, including plume-simulating dilution sampling (PSDS), the distribution of HAPs as a function of particle size, chromium sampling and speciation, and the effect of sootblowing on trace element emissions. 


\subsection{BACKGROUND}

The 1990 Clean Air Act Amendments (CAAA) had two primary goals: pollution prevention and a market-based least-cost approach to emission control. To address air quality issues as well as permitting and enforcement, the 1990 CAAA contain 11 sections, or titles $(1,2)$. The individual amendments included the following:

Title 1 - National Ambient Air Quality Standards

Title II - Mobile Sources

Title III - Hazardous Air Pollutants

Title IV - Acid Deposition Control

Title V - Permits

Title VI - Stratospheric Ozone Protection Chemicals

Title VII - Enforcement

Title VIII - Miscellaneous Provisions

Title IX - Clean Air Research

Title X - Disadvantaged Business Concerns

Title XI - Clean Air Employment Transition Assistance

Titles I, III, IV, and V will change or have the potential to change how operators of coal-fired utility boilers control, monitor, and report emissions. Although the focus of Title $I$ is ambient air quality, it has the potential to impose new regulations on coal-fired utility boilers. Specifically, regions of the country designated as nonattainment for ozone $\left(\mathrm{O}_{3}\right)$, carbon monoxide $(\mathrm{CO})$, respirable particulate, lead $(\mathrm{Pb})$, nitrogen dioxide $\left(\mathrm{NO}_{2}\right)$, or sulfur dioxide $\left(\mathrm{SO}_{2}\right)$ will be under increasing pressure to develop and implement emission reduction plans to achieve ambient air quality standards. Failure to comply can result in sanctions ranging from the cutoff of federal highway funds to severe emission reduction offsets for new sources. As a result, coal-fired utility systems may be targeted for further reductions in 1) the emission of nitrogen species to reduce ambient $\mathrm{O}_{3}$ concentrations and 2 ) fine particulate emissions to reduce ambient respirable particulate concentrations. 
Title III, Hazardous Air Pollutants, requires the U.S. Environmental Protection Agency (EPA) to establish stationary source categories and to implement regulatory standards for 189 air toxics from source categories emitting 25 tons annually of any combination of pollutants or 10 tons annually of a single pollutant. In addition, EPA must issue maximum achievable control technology (MACT) standards. The original list of 189 HAPs may be expanded or reduced based on risk to public health, and once controls are in place, residual risk assessments must be performed to determine whether a need exists for further reductions. Although this amendment requires the regulation of commercial, industrial, and municipal sources, it does not specifically require the regulation of HAPs from utility boilers. Rather, Title III requires that EPA study HAP emissions from utility boilers to determine potential health effects prior to promulgating any new regulations. In addition, a study of mercury $(\mathrm{Hg})$ emissions from utility steam generators, municipal waste combustion units, and other sources was mandated.

Title IV, Acid Deposition Control, and Title V, Permits, have had and will continue to have the most significant effect on coal-fired utility boilers for the remainder of this decade. In the case of Title IV, $\mathrm{SO}_{2}$ emissions must be cut by 10 million tons annually by January 1, 2000, based on 1980 emission estimates, and cannot exceed 8.9 million tons annually in future years. The approach to achieving this requirement consists of two phases. In Phase I, 111 coal-fired plants identified as emitting the most $\mathrm{SO}_{2}$ in the United States were required to reduce their emissions to $2.5 \mathrm{lb}$ of $\mathrm{SO}_{2} / \mathrm{MMBtu}$ by January 1,1995 , for which EPA issued emission allowances. A flexible, market-based decision process involves the issuance of $\mathrm{SO}_{2}$ emission allowances by EPA. These allowances can be bought and sold between companies, transferred within a company, or banked for future use. In Phase II, coal-fired utility boilers will be required to reduce $\mathrm{SO}_{2}$ emissions to $1.2 \mathrm{lb}$ of $\mathrm{SO}_{2} / \mathrm{MMBtu}$ by January 1 , 2000. Again, EPA will issue emission allowances based on the $1.2 \mathrm{lb}$ of $\mathrm{SO}_{2} / \mathrm{MMBtu}$ limit and cap emission allowances at 8.9 million tons annually. To meet emission reporting and compliance requirements, $\mathrm{SO}_{2}$ emission sources are required to install continuous emission monitoring systems. The penalty for emitting $\mathrm{SO}_{2}$ in excess of the emission allowances held is a fee of $\$ 2000 /$ ton. In addition, future $\mathrm{SO}_{2}$ emissions must be offset by an amount equal to the excess emission. Title IV also requires a two- 
million-ton reduction in the emission of nitrogen species by January 1, 2000. Although the approach is expected to be similar to that applied to $\mathrm{SO}_{2}$, specific emission standards have not been established, and the exact implementation schedule is uncertain at this time.

Title V, Permits, will significantly increase the number of regulated sources requiring permits by definition and strengthen state environmental laws. This title defines a major source as one that emits or has the potential to emit 100 tons/year of any criteria pollutants (hydrocarbons, $\mathrm{CO}, \mathrm{Pb}, \mathrm{NO}_{2}, \mathrm{SO}_{2}$, and particulates), 10 tons/year of a HAP, or 25 tons/year of a combination of HAPs. The permit program will be implemented at the state level, with EPA having review authority and the option to intervene if the state program is determined to be inadequate to protect air quality.

In response to the 1990 CAAA requiring the study of HAP emissions from utility boilers, DOE is participating in a collaborative effort with the Utility Air Regulatory Group (UARG), EPA, and the Electric Power Research Institute (EPRI) to establish an appropriate database upon which future regulatory activities can be based. The field sampling efforts are being led primarily by DOE and EPRI, with a few utility companies generating data for their specific systems. EPRI and DOE have provided most of their collected data to EPA.

The EPRI Power Plant Integrated Systems: Chemical Emissions Study (PISCES) research program began in 1988 with the compilation and review of fuel trace element data and HAP emission data in the literature (3). The literature review effort concluded that the available information was inadequate because of insufficient data, the highly variable nature of the existing data, and the fact that the available data had been obtained using inconsistent sampling and analytical procedures. The next step in the PISCES program was to begin the collection of field data to determine the concentration of potential HAPs at various process locations. At this time, sampling activities have been completed at over 24 field sites representing coal- (bituminous, subbituminous, and lignitel, oil-; and gas-fired systems involving a variety of furnace types and pre- and postcombustion emission control strategies (4-6). General conclusions developed as a result of the field sampling activities include.1) that nonvolatile trace elements are 
effectively controlled by conventional particulate control devices such as electrostatic precipitators (ESP) and fabric filters (FF); 2) that nonvolatile trace element emissions can be estimated mathematically based on measured fuel concentrations and particulate control device performance data; 3 ) that conventional flue gas desulfurization (FGD) technology (spray dryers and wet scrubbers) will further reduce the emission of nonvolatile trace elements, effectively control hydrochloric acid $(\mathrm{HCl})$ and, in most cases, effectively control the emission of volatile trace metals such as selenium $(\mathrm{Se})$; 4) that $\mathrm{Hg}$ control has been observed to be highly variable from system to system, with conventional particulate control technology found to be generally ineffective and spray dryers and wet scrubbers observed to be effective occasionally; and 5 ) that flue gas emissions from coal-and oil-fired systems contain low-level concentrations of several organic compounds $\left(\mu \mathrm{g} / \mathrm{Nm}^{3}\right.$ concentrations of volatile organic compounds [VOCs] and aldehydes and $\mathrm{ng} / \mathrm{Nm}^{3}$ concentrations of polycyclic aromatic hydrocarbons [PAHs]). Individual field site reports resulting from the PISCES program have been forwarded to EPA for review as the reports have been completed. A report summarizing the results of the PISCES program has been published by EPRI (7).

The DOE approach to development of a HAP emission database for fossil fuel-fired utility systems has been twofold. The DOE Morgantown Energy Technology Center (METC) has funded HAP-sampling activities at two advanced power system demonstration sites $(8,9)$. These sites represent advanced combustion and gasification technologies and associated gas stream cleanup strategies. One of the sites was the pressurized fluid-bed combustor (PFBC) at the Ohio Power Company Tidd Plant, with results summarized in a report prepared by Radian Corporation (10). In addition to documenting the emissions from the PFBC, this site offered the opportunity to evaluate the ability of a hot-gas advanced particle filter to control HAPs and compare its performance to that of a conventional particulate control device, an ESP. Results showed that the hot-gas advanced particle filter controlled nonvolatile trace element emissions to a greater degree than the ESP, $>99.5 \%$ versus $>95 \%$. This result is directly related to the total average particulate collection efficiencies of the hot-gas advanced particle filter and the ESP, $99.99 \%$ versus $97.2 \%$. Although neither device effectively controlled the emission of most volatile species $\left(\mathrm{HCl}, \mathrm{Cl}_{2}, \mathrm{HF}, \mathrm{Hg}\right.$, and $\left.\mathrm{Se}\right)$, again the hot-gas advanced particle filter performed more efficiently, $<30 \%$ versus 
$<10 \%$. Inlet and outlet data for the hot-gas advanced particle filter also demonstrated variable levels of emission reduction for some specific vapor-phase species: $\mathrm{SO}_{2}$ $(40 \%)$, ammonia (25\%), formaldehyde (94\%), and cyanide $(69 \%)$.

The second DOE METC site is an integrated gasification combined-cycle (IGCC) system operated by Destec Energy Systems located in Plaquemine, Louisiana. This system represents an entrained-flow slagging gasifier processing 2200 tons/day of a western subbituminous coal. This system also permits an evaluation of new emission control concepts and their ability to control HAP emissions. On-site sampling activities and analysis activities have been completed. A report entitled "A Study of Toxic Emissions from a Coal-Fired Gasification Plant" is available from the National Technical Information Service (NTIS).

DOE PETC has focused its efforts on establishing a database for a wide variety of conventional coal-fired systems. Also represented are various conventional and advanced emission control technologies. DOE PETC issued a request for proposals (RFP No. DE-RP22-92PC91349) entitled "Comprehensive Assessment of Toxic Emissions from Coal-Fired Power Plants" on February 10, 1992. As a result of the proposals submitted and the subsequent review process, DOE PETC awarded Phase I contracts to five organizations for HAP sampling at eight utility sites representing nine process configurations. Sampling activities were initiated in 1993, and final project reports were prepared in 1994. A final project report was prepared for each of the nine process configurations by the respective contractors and are available through the NTIS (11-19). This document summarizes key results from the nine process configuration reports with an emphasis on stack-sampling data. 


\subsection{DESCRIPTION OF FIELD TEST PROGRAM}

A primary purpose of the DOE Fossil Energy Program is to foster the continued use of the abundant coal resources in the United States as an efficient and environmentally sound energy source. The DOE accomplishes this objective by supporting the development of technologies that maximize energy efficiency and effectively control the emission of pollutants that are generated as a result of coal utilization. The PETC Flue Gas Cleanup Program was established to develop emission control technologies to promote the continued widespread use of coal in an environmentally acceptable manner. As previously stated, one component of this program involves a collaborative effort between DOE, UARG, EPRI, and EPA to assess the potential for HAP emissions from utility boilers. The PETC role in this collaborative effort is focused on generating HAP data for coal-fired utility systems. The effort consists of two phases. Phase I of the HAP assessment program was to evaluate HAP emissions from eight coal-fired plants and was divided into five tasks: 1) power plant sampling, 2) sample and data analysis, 3) quality assurance/quality control (QA/OC), 4) program coordination, and 5) technology transfer. Phase II was intended to be an option, based on Phase I results, to be exercised by PETC in the event that additional or similar plant configurations would be selected for sampling. At this time, plans are in place to proceed with the Phase II sampling effort involving five additional plants. One plant is an IGCC system; three of the plants represent more conventional power plants utilizing wet scrubbers with one sampling effort being completed (report available $11 / 96)$. The fifth plant is yet to be identified. Completion of the Phase II sampling activities is planned for the second and third quarters of 1997 , with the site reports available in 1998.

\subsection{OBJECTIVES}

The objective of the PETC HAP assessment program, entitled "Comprehensive Assessment of Toxic Emissions from Coal-Fired Power Plants," is to quantify emissions of HAPs associated with coal-fired electric utility systems. Specifically, the assessment addresses the 189 HAPs identified in the 1990 CAAA. Data generated in the study were provided to EPA to assist in meeting the requirements of Title III, Hazardous 
Air Pollutants, to determine what, if any, HAP regulations will be promulgated relative to coal-fired electric utility systems.

Key objectives of the HAP assessment program cover a broad range of technical. issues. Some of these are as follows:

- Generating HAP data for a variety of coal types, furnace types, and emission control systems in order to calculate emission factors for the 189 HAPs identified.

- Determining the effectiveness of commonly used emission control devices (ESPs, FFs, spray dryers, and wet scrubbers) to reduce HAP emissions.

- Determining the effectiveness of advanced emission control devices ladvanced wet FGD for $\mathrm{SO}_{2}$ reduction and selective catalytic reduction [SCR] of nitrogen compounds).

- Calculating subsystem and overall plant material balances for selected HAPs.

- Determining mercury speciation and related emission factors.

- Determining solid-phase HAP distribution as a function of particle size.

- Determining particle-size distribution of stack emissions and ESP/FF hopper ash.

- Evaluating the performance of a stack-sampling method referred to as "plumesimulating dilution sampling (PSDS)."

- Evaluating chromium $(\mathrm{Cr})$ speciation and sampling methods.

- Evaluating the effect of sootblowing on trace element emissions.

- Determining the concentration of HAPs on particle surfaces.

- Determining the distribution of HAPs between solid, liquid, and vapor phases.

The role of the EERC in the overall activity to date has been to provide an independent review of Phase I contractor reports resulting from the assessment activity, make recommendations relative to Phase II activities, and prepare this report summarizing Phase $\mathrm{I}$ results and conclusions in a concise format. 


\subsection{Description Of Plants SAmpled}

Phase I sampling activities were completed on nine different system configurations at eight different power plant sites (11-19). Table 3-1 presents brief descriptive information for each of the nine system configurations, identifying the utility station, unit, station owner, and site contractor. The eight sites represent a range of fuel characteristics, including bituminous, subbituminous, and lignite fuels. Fuel characteristics included $8 \%-20 \%$ ash, $0.6 \%-3.2 \%$ sulfur, $6 \%-38.3 \%$ moisture, and heating values of $6230-12,260 \mathrm{Btu} / \mathrm{lb}$. The geographic locations of the eight plants include the states of Georgia, Ohio (two sites), Illinois, Indiana, Minnesota, North Dakota, and Arizona. Furnace types included tangentially $(t)$-fired, opposed wall-fired, front wall-fired, and cyclone-fired units ranging in size from 75 to $615 \mathrm{MWe}$ gross. Sampling activities were completed while the individual boilers were operated at $\pm 5 \%$ to $10 \%$ of full load. Therefore, the samples collected are representative of normal full-load boiler operating conditions.

Electrostatic precipitators were used to control particulate emissions on six of the nine system configurations. Specific collection area (SCA) for these units ranged from 180 to $750 \mathrm{ft}^{2} / 1000$ actual cubic feet per minute (acfm) resulting in particulate collection efficiencies of nominally $97 \%$ to $99.8 \%$, which represents marginal to highly efficient ESP control technology. Fabric filters were used to control particulate emissions on three of the nine system configurations: two reverse-gas units and one slipstream pulse-jet baghouse. Particulate collection efficiency ranged from nominally $99.8 \%$ to $99.98 \%$ for filter face velocities of nominally 4.5 and $2 \mathrm{ft} / \mathrm{min}$, respectively.

Sulfur dioxide control technologies were used on five of the nine process configurations. These included a lime-based spray dryer system, a conventional limestone wet FGD system, the Chiyoda Thoroughbred-121 FGD process, the Pure Air advanced FGD system, and a slipstream demonstration of the $\mathrm{SNOX} \mathrm{SO}_{2}$-to- $\mathrm{SO}_{3} /$ acid condensation process. Sulfur dioxide control ranged from $60 \%$ for the spray dryer system to $>90 \%$ for the wet-scrubbing concepts. The SNOX process demonstrated the highest level of $\mathrm{SO}_{2}$ control, $94 \%$ to $96 \%$.

No control systems for nitrogen species were represented on six of the nine process configurations. Two systems were t-fired units using overfire air to reduce the 
Table 3-1. Field Site Descriptions for DOE Phase I HAP Sampling ${ }^{a}$

\begin{tabular}{|c|c|c|c|c|c|}
\hline Contractor & $\begin{array}{l}\text { Radian Corporation } \\
8501 \text { N Mo-Pac Boulevard } \\
\text { Box } 201088 \\
\text { Austin, TX 78720-1088 } \\
\text { W.A. Williams } \\
\text { (512) 454-4797 }\end{array}$ & $\begin{array}{l}\text { Energy and Environmental Research } \\
\text { Corporation (EER) } \\
18 \text { Mason } \\
\text { Irvine, CA } 92718 \\
\text { Glenn C. England } \\
\text { (714) } 859-8851\end{array}$ & $\begin{array}{l}\text { Battelle Columbus Operations } \\
505 \text { King Avenue } \\
\text { Columbus, } \mathrm{OH} 43201 \\
\\
\text { George M. Sverdrup } \\
(614) 424-5014\end{array}$ & & \\
\hline Field Site & $\begin{array}{l}\text { Georgia Power Company } \\
\text { Plant Yates } \\
\text { Unit No. } 1 \\
\text { Newnan, GA } \\
\end{array}$ & $\begin{array}{l}\text { Ohio Power Company } \\
\text { Cardinal Station } \\
\text { Unit No. } 1 \\
\text { Brilliant, OH } \\
\end{array}$ & $\begin{array}{l}\text { Cooperative Power } \\
\text { Association } \\
\text { Coal Creek Station Unit No. } 1 \\
\text { Underwood, ND } \\
\end{array}$ & $\begin{array}{l}\text { Ohio Edison Company } \\
\text { Niles Station } \\
\text { Unit No. } 2 \\
\text { Niles, OH } \\
\end{array}$ & $\begin{array}{l}\text { Ohio Edison Company } \\
\text { Niles Station } \\
\text { Unit No. } 2 \\
\text { Niles, OH } \\
\end{array}$ \\
\hline $\begin{array}{l}\text { Furnace Type } \\
\text { Year }\end{array}$ & $\begin{array}{c}\text { Combustion Engineering } \\
\text { wet-bottom t-fired furnace } \\
1950\end{array}$ & $\begin{array}{c}\text { Babcock \& Wilcox dry-bottom opposed wall- } \\
\text { fired furnace, cell burners } \\
1967\end{array}$ & $\begin{array}{c}\text { Combustion Engineering dry- } \\
\text { bottom, t-fired, divided furnace } \\
1979\end{array}$ & $\begin{array}{l}\begin{array}{l}\text { Babcock \& } \\
\text { furnace }\end{array} \\
1954\end{array}$ & $\begin{array}{l}\begin{array}{l}\text { Babcock \& } \text { Wilcox cyclone } \\
\text { furnace }\end{array} \\
1954\end{array}$ \\
\hline $\begin{array}{l}\text { Boiler Size, } \\
\text { gross/net }\end{array}$ & $105 / 100 \mathrm{MWe}_{\mathrm{e}}$ & $615 / 590 \mathrm{MWe}_{\mathrm{e}}$ & $550 / 506 \mathrm{MWe}$ & $115 / 108 \mathrm{MWe}_{\mathrm{\theta}}$ & $115 / 108 \mathrm{MWe}_{\mathrm{e}}$ \\
\hline Fuel $^{b}$ & IL Nos. 5 and 6 bituminous blend & Pittsburgh No. 8 bituminous (Windsor mine) & ND lignite (Falkirk mine) & PA-OH bituminous blend & PA-OH bituminous blend \\
\hline Ash, \% & 12.2 & 11.8 & 10.4 & 11.0 & 10.9 \\
\hline Sulfur, $\%$ & 2.9 & 3.0 & 0.7 & 2.8 & 2.6 \\
\hline $\mathrm{H}_{2} \mathrm{O}, \%$ & 11.7 & 6.9 & 38.3 & 6.5 & 5.9 \\
\hline Btu/lb & 11,200 & 12,200 & 6230 & 12,090 & 12,260 \\
\hline \begin{tabular}{|l} 
Particulate \\
Control
\end{tabular} & Buell ESP, 4 fields, 12 chambers & $\begin{array}{l}\text { Two parallel Research-Cottrell ESPs (A and } \\
\text { B), } 10 \text { fields each }\end{array}$ & $\begin{array}{l}\text { Wheelabrator-Frye, Inc., ESP, } \\
2 \text { units in parallel, } 48 \text { fields }\end{array}$ & $\begin{array}{l}\text { Wheelabrator-Frye, Inc., ESP, } \\
5 \text { fields }\end{array}$ & $\begin{array}{l}35-M W \text { FG slipstream, SNOX } \\
\text { pulse-jet/baghouse, } \\
6 \text { compartments }\end{array}$ \\
\hline A/C Ratio & -- & -. & .. & -- & 4.51 net \\
\hline $\begin{array}{l}\text { SCA } \\
\text { Control, \% }\end{array}$ & $\begin{array}{c}210 \\
98.2-98.7 \\
\end{array}$ & $\begin{array}{c}424 \\
99-99.5 \\
\end{array}$ & $\begin{array}{r}599 \\
99.5 \\
\end{array}$ & $\begin{array}{c}750 \\
97.8-99.2 \\
\end{array}$ & 99.79 \\
\hline Ash Handling & $\begin{array}{l}\text { Bottom/ESP/economizer ash (wet } \\
\text { sluice, settling pond) }\end{array}$ & Bottom/ESP ash (wet sluice, settling ponds) & $\begin{array}{l}\text { Bottom ash/economizer } \\
\text { ash/FGD solids (wet sluice, } \\
\text { pond), ESP ash (dry) }\end{array}$ & Wet sluice, settling pond & Wet sluice, settling pond \\
\hline $\mathrm{SO}_{2}$ Control & $\begin{array}{l}\text { Chiyoda Thoroughbred-121 FGD, } \\
\text { single-jet bubbling reactor, gypsum by- } \\
\text { product, } \\
88.8 \%-93.0 \% \text { control }\end{array}$ & None & $\begin{array}{l}\text { Combustion Engineering lime- } \\
\text { based/wet FGD, } 4 \text { spray } \\
\text { towers } / 40 \% \text { bypass flue gas } \\
\text { reheat, } 90 \% \text { control on } 60 \% \\
\text { flue gas }\end{array}$ & None & $\begin{array}{l}\text { 35-MWe FG slipstream, SNOX, } \\
\mathrm{SO}_{2} \text {-to-SO } \mathrm{SO}_{3} \text { oxidation, acid } \\
\text { condenser, } 94 \%-96 \% \text { control }\end{array}$ \\
\hline $\mathrm{NO}_{x}$ Control & t-firing & Nane & $t$-firing/overfire air & None & $\begin{array}{l}\text { 35-MWa FG slipstream, SNOX } \\
\mathrm{NH}_{3} / \mathrm{SCR}, 85 \%-93 \% \text { control }\end{array}$ \\
\hline
\end{tabular}

Continued ... 
Table 3-1 (continued)

\begin{tabular}{|c|c|c|c|c|c|}
\hline Contact & $\begin{array}{l}\text { Roy F. Weston, Inc. } \\
\text { One Weston Way, Building } 5 \\
\text { West Chester, PA 19380-1499 } \\
\text { Barry L. Jackson } \\
(610) 701-7215 \\
\end{array}$ & & $\begin{array}{l}\text { Southern Research Institute } \\
2000 \text { 9th Avenue South } \\
\text { PO Box 55305 } \\
\text { Birmingham, AL 35255-5305 } \\
\text { Vann Bush } \\
\text { (205) 581-2269 }\end{array}$ & & . \\
\hline Field Site & $\begin{array}{l}\text { Minnesota Power Company } \\
\text { Boswell Energy Center } \\
\text { Unit No. } 2 \\
\text { Cohasset, MN }\end{array}$ & $\begin{array}{l}\text { Illinois Power Company } \\
\text { Baldwin Power Station } \\
\text { Unit No. } 2 \\
\text { Baldwin, IL }\end{array}$ & $\begin{array}{l}\text { Tucson Electric Power Company } \\
\text { Springerville Generating Station } \\
\text { Unit No. } 2 \\
\text { Springerville, AZ } \\
\end{array}$ & $\begin{array}{l}\text { Northern Indiana Public Ser } \\
\text { Bailly Station } \\
\text { Unit Nos. } 7 \text { and } 8 \\
\text { Chesterton, IN } \\
\end{array}$ & Company \\
\hline Furnace Type & $\begin{array}{l}\begin{array}{l}\text { Riley Stoker dry-bottom, front-fired } \\
\text { furnace }\end{array} \\
1957 \\
\end{array}$ & $\begin{array}{c}\text { Babcock \& Wilcox cyclone furnace } \\
1973 \\
\end{array}$ & $\begin{array}{l}\begin{array}{l}\text { Combustion Engineering dry-bottom, } \\
t \text {-fired furnace } \\
1990 \\
\end{array} \\
\end{array}$ & $\begin{array}{c}\text { Babcock \& Wilcox cyclone } \\
\frac{\text { Unit No. } 7}{1962}\end{array}$ & $\frac{\text { Unit No. } 8}{1968}$ \\
\hline $\begin{array}{l}\text { Boiler Size, } \\
\text { gross/net }\end{array}$ & $75 / 69 \mathrm{MWe}$ & $595 / 568 \mathrm{MW}_{\theta}$ & $422 / 383 \mathrm{MW}_{\mathrm{\theta}}$ & $\frac{\text { Unit } \mathrm{No} .7}{175 / 160 \mathrm{MW}}$ & $\frac{\text { Unit No. } 8}{345 / 320 \mathrm{MWe}}$ \\
\hline $\begin{array}{l}\text { Fuel }^{b} \\
\text { Ash, \% } \\
\text { Sulfur, \% } \\
\mathrm{H}_{2} \mathrm{O}, \% \\
\text { Btu/lb } \\
\end{array}$ & \begin{tabular}{|c|} 
PRB $^{\mathrm{C}}$ subbituminous (Big Sky mine) \\
8.4 \\
0.7 \\
24.8 \\
8,800 \\
\end{tabular} & $\begin{array}{r}\text { IL bituminous blend } \\
10.2 \\
2.9 \\
15.0 \\
10,600 \\
\end{array}$ & \begin{tabular}{|c} 
NM subbituminous (Lee Ranch mine) \\
19.6 \\
0.6 \\
12.0 \\
9450 \\
\end{tabular} & IL/IN Basin bituminous ICa & $\begin{array}{l}n \text { mine) } \\
0.7 \\
3.2 \\
0.2 \\
.100 \\
\end{array}$ \\
\hline $\begin{array}{l}\text { A/C Ratio } \\
\text { SCA } \\
\text { Control, \% }\end{array}$ & $\begin{array}{l}\text { Joy-Western Precipitation baghouse, } \\
8 \text { compartments, reverse-gas }\end{array}$ & \begin{tabular}{|c|} 
Joy-Western Precipitation ESP, 6 \\
chambers, 4 fields \\
\\
.. \\
180 \\
$95.6-98.1$
\end{tabular} & $\begin{array}{c}\text { Joy-Niro baghouses, } 2 \text { parallel units, } \\
28 \text { compartments, reverse-gas } \\
\\
2.09 \\
- \\
99.98\end{array}$ & $\begin{array}{l}\text { Unit } \mathrm{No} .7 \\
\text { Wheelabrator-Frye, Inc. } \\
\text { single ESP, } 12 \text { fields, } \mathrm{NH}_{3} \\
\text { injection } \\
-. \\
354 \\
\text { NA } \\
\end{array}$ & $\begin{array}{l}\text { Unit No. } 8 \\
\text { Wheelabrator-Frye, Inc. } \\
\text { two ESPs } 12 \text { fields/each, } \mathrm{NH}_{3} \\
\text { injection } \\
\begin{array}{c}-- \\
387 / \text { ea. } \\
99.8\end{array}\end{array}$ \\
\hline Ash Handling & $\begin{array}{l}\text { Bottom ash (wet sluice, settling } \\
\text { pond), economizer/baghouse ash (dry) }\end{array}$ & Wet sluice, settling pond & $\begin{array}{l}\text { Bottom ash/economizer ash/pyrite } \\
\text { rejects (wet sluice, dewatering tanks), } \\
\text { baghouse ash (dry) }\end{array}$ & $\begin{array}{l}\text { Bottom/economizer ash (w } \\
\text { (dry) }\end{array}$ & luice, settling pond), ESP ash \\
\hline $\mathrm{SO}_{2}$ Control & None & None & $\begin{array}{l}\text { Joy-Niro spray dryers, } 3 \text { absorbers, } 60 \% \\
\text { control, } 13 \% \text { flue gas bypass reheat } \\
\end{array}$ & $\begin{array}{l}\text { Pure Air advanced FGD, sir } \\
93 \% \text { control }\end{array}$ & bsorber, gypsum by-product, \\
\hline $\mathrm{NO}_{x}$ Control & None & None & t-firing/overfire air & & lone \\
\hline
\end{tabular}

Information included in this table was obtained from the site reports prepared by the contractors $(11-19)$

$\omega$ Fuel blends are indicated when the fuel source includes multiple mines. The fuels identified were the fuels used at the time of the field tests. Current fuel use may have changed in some cases. 
emission of nitrogen species. However, $\mathrm{NO}_{x}$ reduction data were not reported. The SNOX slipstream demonstration system reported achieving $85 \%-93 \% \mathrm{NO}_{x}$ control using ammonia injection with an SCR catalyst.

\subsection{Description Of SAmpling, Analytical, and QA/QC Methods}

Sample collection for each of the nine system configurations varied somewhat from site to site because of site-specific characteristics. However, a thorough sampling approach was applied at each site to ensure that sufficient solid-, liquid-, and gas-phase samples were collected to complete the HAP assessment. Table 3-2 presents a generic list of sample types and locations indicative of the effort completed at each field site. Considering that efforts were made to sample several locations simultaneously and collection of triplicate samples was desired, the variety of sample types identified in the table illustrates the extensive nature of the overall sampling effort. Solid samples included various coal, ash, and FGD by-product/waste samples from dry and slurry/sluice streams. Liquid samples included various water and slurry/sluice streams. Gas samples were generally limited to flue gas at the inlet and outlet of the particulate control device and in the stack.

Table 3-2. Sample Types and Locations Generic to the HAP Assessment Effort

\section{Solid Samples}

Raw Coal

Rejects from Crusher/Pulverizer

Economizer Ash

Hopper Ash from Each ESP Field

Fly Ash at ESP/FF Outlet

Raw Limestone/Sorbent

FGD By-Product/Waste Solids

\section{Liquid Samples}

Plant Makeup Water (possibly multiple sources)

Cooling Tower Blowdown

Economizer Sluice Water

Inlet/Outlet Condenser Water

Coal Prep. Inlet/Outlet Water

FGD Slurry Blowdown

FGD By-Product/Waste Slurry

\section{Gas Samples}

ESP/FF Flue Gas Inlet/Outlet

Spray Dryer Inlet/Outlet
Coal Feed to Boiler

Bottom Ash/Slag from Boiler

Fly Ash at ESP/FF Inlet

FF Hopper Ash

Ash to Settling Pond

FGD Limestone/Sorbent Feed Slurry

Fly Ash in Stack Flue Gas

Coal Pile Runoff

Boiler Sluice Water

ESP/FF Sluice Water

Settling Pond Recycle Water

FGD Makeup Water

FGD Slurry Inlet

FGD Recycle Water
Wet FGD Inlet/Outlet

Stack Flue Gas 
Sampling methods for solids varied somewhat from location to location because of the type of sample to be collected, access limitations, and quantity of sample required. However, all solid samples were collected in order to obtain time-averaged composites for specifically defined sampling periods. Actual solid-sampling methods included periodic grab samples from bulk solid streams such as coal and various ash and waste sources, as well as isokinetic flue gas sampling with multicyclones and filters for entrained fly ash. Grab samples of bulk liquids were also collected to form composites to provide time-averaged samples for specifically defined sampling periods. Gas samples for vapor species and entrained solids were typically collected using isokinetic sampling methods such as EPA Methods 5, 17, 26A, 29, 0010/23, and 0013. Nonisokinetic sampling methods included EPA Methods $3 \mathrm{~A}, 6 \mathrm{C}, 7 \mathrm{E}, 10,18$, $25 \mathrm{~A}$, and 26 and the volatile organic sampling train (VOST). Process stream volumetric and/or mass flow rates were determined for each sample location in order to permit the calculation of mass balances. The contractors discussed in detail the sampling, measurement, and analytical methodologies used in their respective site reports or referenced the appropriate EPA methods in their reports.

Table 3-3 lists the critical target analytes for which sampling and analysis were specifically requested in the PETC RFP. The original list includes trace elements, radionuclides, anions, inorganic compounds, and organic compounds considered critical to meeting program objectives. Several major elements were added to the list in order to permit a more thorough evaluation of mass balance results for trace elements. Table 3-4 identifies the variety of analytical methods used to quantify critical target analytes. In addition, the PETC RFP requested that, within reason, the sampling and analytical approach should be developed to permit the quantitative determination of any of the 189 HAPs listed in the 1990 CAAA that may be detected as a result of the assessment effort. Table 3-5 lists the sampling and analytical contractors and subcontractors. Table 3-6 presents the 189 HAPs listed in the CAAA, plus hydrogen sulfide, which was included in the PETC list.

Quality assurance/quality control measures were a critical aspect of the overall program in order to ensure that meaningful data were obtained. Site-specific QA/OC plans were developed by each contractor to address sample collection, sample handling, 
Table 3-3. Critical Target Analytes for Which Sampling and Analysis Were Specifically Requested

\begin{tabular}{|c|c|c|c|}
\hline \multicolumn{4}{|l|}{ Major Elements } \\
\hline Aluminum (Al) & Calcium (Ca) & Iron (Fe) & Magnesium (Mg) \\
\hline Potassium (K) & Sodium (Na) & Silicon (Si) & Strontium (Sr) \\
\hline \multicolumn{4}{|l|}{ Titanium (Ti) } \\
\hline \multicolumn{4}{|l|}{ Trace Elements } \\
\hline Antimony (Sb) & Arsenic (As) & Barium (Ba) & Beryllium (Be) \\
\hline Boron (B) & Cadmium (Cd) & Chromium (Cr) & Cobalt (Co) \\
\hline Copper (Cu) & Lead $(\mathrm{Pb})$ & Manganese $\{\mathrm{Mn}\rangle$ & Mercury $(\mathrm{Hg})$ \\
\hline Molybdenum (Mo) & Nickel (Ni) & Selenium (Se) & Vanadium (V) \\
\hline \multicolumn{4}{|l|}{ Anions } \\
\hline Phosphates & Hydrochloric Acid ( $\mathrm{HCl})$ & Hydrogen Fluoride $(\mathrm{HF})$ & Sulfates \\
\hline \multicolumn{4}{|l|}{ Reduced Species } \\
\hline Ammonia & Cyanide & & \\
\hline \multicolumn{4}{|l|}{ Organics } \\
\hline Benzene & Dioxins & Formaldehyde & Furans \\
\hline \multicolumn{2}{|c|}{ Polycyclic Organic Matter } & Toluene & \\
\hline Radionuclides & & & \\
\hline
\end{tabular}

sample analyses, data analyses, and specific corrective action to be taken when preestablished specifications were not met. In addition to the procedures established by the individual contractors for QA/QC audits, independent QA/QC audits funded by EPA were performed by Research Triangle Institute (RTI). Examples of the various QA/QC procedures employed included field blanks, trip blanks, spikes, documentation of detection limits, and round-robin analyses of the various coals encountered by the contractors during the Phase 1 study. Extensive performance and technical audits were performed at all of the sites by RTI. 
Table 3-4. Analytical Techniques Used to Detect and Quantify Critical Target Analytes

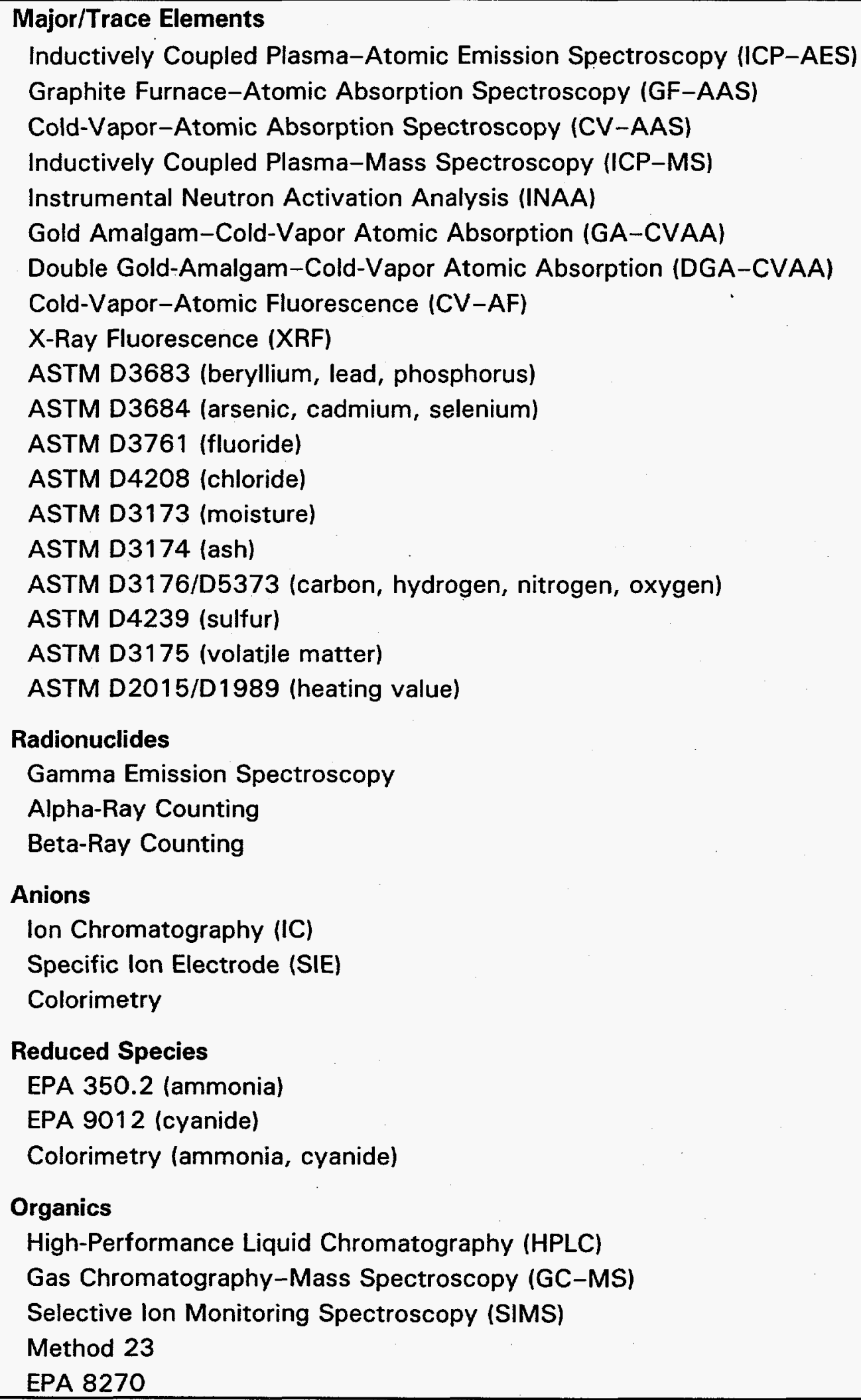

\section{Organics}

High-Performance Liquid Chromatography (HPLC)

Gas Chromatography-Mass Spectroscopy (GC-MS)

Selective Ion Monitoring Spectroscopy (SIMS)

Method 23

EPA 8270 
Table 3-5. Sampling and Analytical Contractors and Subcontractors

\section{Battelle - Columbus Operations \\ Chester Environmental \\ Zande Environmental Services \\ Commercial Testing and Engineering Company \\ International Technology Corporation \\ Element Analysis Corporation \\ RTI}

Southern Research Institute (SRI)

Guardian Systems, Inc.

Commercial Testing and Evaluation, Inc.

Core Laboratories, Inc.

Brooks Rand, Ltd.

RTI

Roy F. Weston, Inc.

CARNOT, Inc.

CONSOL INC

Triangle Laboratories, Inc.

TMA Eberline

Frontier Geosciences

RTI

Radian

ADA Technologies

Commercial Testing and Engineering Company

North Carolina State University

Harvard University

Triangle Laboratories, Inc.

University of Utah

Charles Evans and Associates

RTI

Energy and Environmental Research Corporation (EER)

DEECO

International Technology Corporation: Oak Ridge National Laboratories

North Carolina State University

Commercial Testing and Engineering Company

Twin Cities Testing

Air Toxics Limited

ETS International, Inc.

RTI 
Table 3-6. HAPs listed in the 1990 CAAA

\begin{tabular}{|c|c|c|}
\hline $\begin{array}{l}\text { Acetaldehyde } \\
\text { Acetamide }\end{array}$ & $\begin{array}{l}\text { Dimethyl formamide } \\
\text { 1,1-Dimethyl hydrazine }\end{array}$ & $\begin{array}{l}\text { Pentachloronitrobenzene } \\
\text { (Quintobenzene) }\end{array}$ \\
\hline Acetonitrile & Dimethyl phthalate & Pentachlorophenol \\
\hline Acetophenone & Dimethyl sulfate & Phenol \\
\hline 2-Acetylaminofluorene & 4,6-Dinitro-o-cresol and salts & p-Phenylenediamine \\
\hline Acrolein & 2,4-Dinitrophenol & Phosgene \\
\hline Acrylamide & 2,4-Dinitrotoluene & Phosphine \\
\hline Acrylic acid & 1,4-Dioxane (1,4-Diethyleneoxide) & Phosphorus \\
\hline Acrylonitrile & 1,2 -Diphenylhydrazine & Phthalic anhydride \\
\hline Allyl chloride & Epichlorohydrin & Polychlorinated biphenyls (Aroclors) \\
\hline 4-Aminobipheny! & (1-Chloro-2,3-epoxypropane) & 1,3-Propane sultone \\
\hline Aniline & 1,2-Epoxybutane & Beta-Propiolactone \\
\hline$o$-Anisidine & Ethyl acrylate & Propionaldehyde \\
\hline Asbestos & Ethyl benzene & Propoxur (Baygon) \\
\hline $\begin{array}{l}\text { Benzene } \\
\quad \text { (including benzene from gasoline) }\end{array}$ & $\begin{array}{l}\text { Ethyl carbamate (Urethane) } \\
\text { Ethyl chloride (Chloroethane) }\end{array}$ & $\begin{array}{l}\text { Propylene dichloride } \\
\text { (1,2-Dichloropropane) }\end{array}$ \\
\hline Benzidine & Ethylene dibromide (Dibromoethane) & Propylene oxide \\
\hline Benzotrichloride & Ethylene dichlorlde & 1,2-Propyleneimine (2-Methyl aziridine) \\
\hline Benzyl chloride & (1,2-Dichloroethane) & Quinoline \\
\hline Biphenyl & Ethylene glycol & Quinone \\
\hline Bis (2-ethylhexyl)phthalate (DEHP) & Ethylene imine (Aziridine) & Styrene \\
\hline Bis(chloromethyl)ether & Ethylene oxide & Styrene oxide \\
\hline Bromoform & Ethylene thiourea & $2,3,7,8$-Tetrachlorodibenzo-p-dioxin \\
\hline 1,3-Butadiene & Ethylidene dichloride & $1,1,2,2$-Tetrachloroethane \\
\hline Calcium cyanamide & (1,1-Dichloroethane $\}$ & Tetrachloroethylene \\
\hline Caprolactam & Formaldehyde & (Perchloroethylene) \\
\hline Captan & Heptachlor & Titanium tetrachloride \\
\hline Carbaryl & Hexachlorobenzene & Toluene \\
\hline Carbon disulfide & Hexachlorobutadiene & 2,4-Toluene diamine \\
\hline Carbon tetrachloride & Hexachlorocyclopentadiene & 2,4-Toluene diisocyanate \\
\hline Carbonyl sulfide & Hexachloroethane & $o$-Toluidine \\
\hline Catechol & Hexamethylene-1,6-diisocyanate & Toxaphene (chlorinated camphene) \\
\hline Chloramben & Hexamethylphosphoramide & 1,2,4-Trichlorobenzene \\
\hline Chlordane & Hexane & 1,1,2-Trichloroethane \\
\hline Chlorine & Hydrazine & Trichloroethylene \\
\hline Chloroacetic acid & Hydrochloric acid & 2,4,5-Trichlorophenol \\
\hline 2-Chloroacetophenone & Hydrogen fluoride (Hydrofluoric acid) & $2,4,6$-Trichlorophenol \\
\hline Chlorobenzene & Hydrogen sulfide & Triethylamine \\
\hline Chlorobenzilate & Hydroquinone & Triffuralin \\
\hline Chloroform & Isophorone & 2,2,4-Trimethylpentane \\
\hline Chloromethyl methyl ether & Lindane (all isomers) & Vinyl acetate \\
\hline Chloroprene & Maleic anhydride & Vinyl bromide \\
\hline Cresols/Cresylic acid & Methanol & Vinyl chloride \\
\hline (isomers and mixture) & Methoxychlor & Vinylidene chloride \\
\hline$o$-Cresol & Methyl bromide (Bromomethane) & (1,1-Dichloroethylene) \\
\hline$m$-Cresol & Methyl chloride (Chloromethane) & Xylenes (isomers and mixture) \\
\hline$\rho$-Cresol & Methyl chloroform & $o$-Xylenes \\
\hline Cumene & (1,1,1-Trichloroethane) & $m$-Xylenes \\
\hline 2,4-D, salts and esters & Methyl ethyl ketone (2-Butanone) & $\rho$-Xylenes \\
\hline DDE & Methyl hydrazine & Antimony compounds \\
\hline Diazomethane & Methyl iodide (lodomethane) & Arsenic compounds \\
\hline Dibenzofurans & Methyl isobutyl ketone (Hexone) & (inorganic including arsine) \\
\hline 1,2-Dibromo-3-chloropropane & Methyl isocyanate & Beryllium compounds \\
\hline Dibutylphthalate & Methyl methacrylate & Cadmium compounds \\
\hline 1,4-Dichlorobenzene(p) & Methyl tert butyl ether & Chromium compounds \\
\hline 3,3-Dichlorobenzidene & 4,4-Methylene bis(2-chloroaniline) & Cobalt compounds \\
\hline Dichloroethyl ether & Methylene chloride (Dichloromethane) & Coke oven emissions \\
\hline (Bis[2-chloroethyl]ether) & Methylene diphenyl diisocyanate (MDI) & Cyanide compounds \\
\hline 1,3-Dichloropropene & 4,4'-Methylenedianiline & Glycol ethers \\
\hline Dichlorvos & Naphthalene & Lead compounds \\
\hline Diethanolamine & Nitrobenzene & Manganese compounds \\
\hline $\mathrm{N}, \mathrm{N}$-Diethyl aniline & 4-Nitrobiphenyl & Mercury compounds \\
\hline (N,N-Dimethylaniline) & 4-Nitrophenol & Fine mineral fibers \\
\hline Diethyl sulfate & 2-Nitropropane & Nickel compounds \\
\hline 3,3-Dimethoxybenzidine & $\mathrm{N}$-Nitroso-N-methylurea & Polycylic organic matter \\
\hline Dimethyl aminoazobenzene & N-Nitrosodimethylamine & Radionuclides (including radon) \\
\hline 3,3'-Dimethyl benzidine & N-Nitrosomorpholine & Selenium compounds \\
\hline Dimethyl carbamoyl chloride & Parathion & \\
\hline
\end{tabular}




\subsection{RESULTS AND DISCUSSION OF PHASE I DATA}

A brief summary of a broad number of technical areas is presented in the following sections of this document. These technical areas include coal analyses, emission factors for inorganic, organic, radionuclide, and acid gas-halogen elements and species, and several special topics. Coal analysis data are discussed in terms of individual plant data and round-robin results. Inorganic, organic, radionuclide, and acid gas-halogen data are primarily discussed in terms of stack concentration and emission factors. Some limited discussion is also included concerning percent penetration data for inorganic trace elements and acid gases-halogens. Special topics include 1) PSDS, 2) HAP distribution as a function of particle size, 3) particle-size distribution of stack emissions and ESP/FF hopper ash, 4) chromium speciation and sampling, 5) dioxin and furan reduction across ESPs, 6) the effect of sootblowing on trace element emissions, and 7) the effect of HAPs on particle surfaces.

Since the basis of most of the discussion will focus on emission factors, it is important to explain what emission factors are and how they are calculated. Emission factors are estimates of emissions reported on a pound per trillion Btu ( $\left.\mathrm{lb} / 10^{12} \mathrm{Btu}\right)$ of heat input basis. Emission factor values are calculated by dividing the mass flow of an element or compound ( $\mathrm{lb} / \mathrm{hr})$ by the product of the coal feed rate and the coal higher heating value $(\mathrm{lb} / \mathrm{hr} \times \mathrm{Btu} / \mathrm{lb})$ with appropriate unit conversions. The mass flow rate of an element or compound in the stack is calculated from the measured concentration on a dry basis and normalized to $3 \% \mathrm{O}_{2}\left(\mu \mathrm{g} / \mathrm{Nm}^{3}\right.$, based on $0^{\circ} \mathrm{C}$ and 1 atmosphere) and the flue gas volumetric flow rate $\left(\mathrm{Nm}^{3} / \mathrm{hr}\right)$ with appropriate unit conversions. The purpose of calculating emission factors is to provide a simple method for estimating annual HAP emissions on a ton per year basis.

Percent penetration was calculated from the mean stack concentration (determined as a result of field measurements) divided by the theoretical stack concentration based on the assumption that $100 \%$ of the element or compound in the coal exits the stack. Penetration data were calculated for inorganic trace elements and acid gases-halogens for the purpose of evaluating the relative performance of emission control technologies. 


\subsection{Round-Robin Coal ANalyses}

Trace elements in coal are defined as those elements that occur in concentrations of no more than $100 \mu \mathrm{g} / \mathrm{g}$. Eleven trace elements are listed in the 1990 CAAA as HAPs. The interlaboratory reproducibility of trace element analyses were quite large (20) and must be considered when potential emissions from coal-fired power plants are evaluated. In order to better define the analytical variability that can be expected for trace elements, a coal analysis round-robin study was included in the DOE Phase I effort. CONSOL INC coordinated the study. Much of the material in this section was drawn from its report to DOE (21).

Seventeen trace elements were included in the round-robin study, including all those listed as HAPs in the 1990 CAAA. Other analyses included in the round-robin program were proximate-ultimate and ten major ash elements, as listed in Table 4-1.

Table 4-1. Parameters Tested in the Round-Robin Study

\begin{tabular}{ll}
\hline Analysis Type & Parameter \\
\hline Proximate-Ultimate & Moisture, ash, $\mathrm{C}, \mathrm{H}, \mathrm{N}, \mathrm{S}, \mathrm{Cl}$, heating value \\
Major Ash Elements & $\mathrm{Al}_{2} \mathrm{O}_{3}, \mathrm{CaO}, \mathrm{Fe}_{2} \mathrm{O}_{3}, \mathrm{MgO}, \mathrm{P}_{2} \mathrm{O}_{5}, \mathrm{~K}, \mathrm{O}, \mathrm{SiO}_{2}, \mathrm{Na}_{2} \mathrm{O}, \mathrm{SO}_{3}, \mathrm{TiO}_{2}$ \\
Trace Elements & $\mathrm{Sb}^{\mathrm{a}}, \mathrm{As}^{\mathrm{a}}, \mathrm{Ba}, \mathrm{Be}^{\mathrm{a}}, \mathrm{B}, \mathrm{Cd}^{\mathrm{a}}, \mathrm{Cr}^{\mathrm{a}}, \mathrm{Co}^{\mathrm{a}}, \mathrm{Cu}, \mathrm{Pb}^{\mathrm{a}}, \mathrm{Mn}^{\mathrm{a}}, \mathrm{Hg}^{\mathrm{a}}, \mathrm{Mo}^{\mathrm{a}} \mathrm{Ni}^{\mathrm{a}}, \mathrm{Se}^{\mathrm{a}}$, \\
\hline & $\mathrm{V}, \mathrm{F}$ \\
\hline L Listed as a HAP in the &
\end{tabular}

The coal samples used in the round-robin study were supplied to CONSOL INC by the primary contractors at each of the eight utility test sites. Every feed coal used in Phase I was thus represented in the round-robin study. Round-robin samples were obtained following the field test period, because the round-robin study was not included in the initial plans for Phase I. The round-robin coal samples thus may not actually represent the feed coal used during the Phase I testing. As shown in Table 4-2, the coals used included two lllinois Basin bituminous coals, three mid-sulfur bituminous coals, one PRB subbituminous coal, a New Mexico subbituminous coal, and one lignite. Samples were prepared following American Society for Testing and Materials (ASTM) 
Table 4-2. Fuels Included in the Round-Robin Study

\begin{tabular}{lccc}
\hline Site & Primary Contractor & Feed Coal & Coal Type \\
\hline Coal Creek & Battelle & Falkirk (North Dakota) & Lignite \\
Niles/SNOX & Battelle & Pennsylvania - Ohio blend & Bituminous \\
Cardinal & EER & Pittsburgh No. 8 & Bituminous \\
Yates & Radian & Illinois No. 5 and No. 6 blend & Bituminous \\
Bailly & SRI & Illinois-Indiana Basin & Bituminous \\
Springerville & SRI & Lee Ranch (New Mexico) & Subbituminous \\
Baldwin & Weston & Illinois blend & Bituminous \\
Boswell & Weston & Big Sky (PRB) & Subbituminous \\
\hline
\end{tabular}

Method D2013, Standard Method of Preparing Coal Samples for Analyses (22). A spinning riffle was used to divide each coal sample into several homogeneous splits.

\subsubsection{Scope of Round-Robin Program}

Five contractors participated in the round-robin study using the same laboratories that were contracted to perform the field test analyses, as shown in Table 3-5. Each participating laboratory received duplicate samples of each of the eight feed coals, along with a sample of a certified reference coal (SRM 1632b, a Pittsburgh seam coal) from the National Institute for Standards and Technology (NIST). All samples were identified by code letters only. The laboratories were requested to analyze each sample in duplicate with the same procedures used in analyzing samples from Phase I testing. The round-robin study was thus designed to allow the determination of intralaboratory repeatability, interlaboratory reproducibility, and accuracy based on a NIST standard.

All of the laboratories used ASTM standard methods for the proximate and ultimate analyses. None of the other parameters were measured using the same technique by all laboratories. Techniques used included GF-AAS, ICP-AES, ICP-MS, INAA, IC, CV-AF, and XRF. Techniques used to measure mercury included GA-CVAA, DGA-CVAA, and CV-AF. 


\subsubsection{Contractors' Results}

Results for each contractor are summarized in Tables 4-3 through 4-8. All of the contractors included the round-robin results in their reports. However, only EER (Cardinal Station) and Battelle (Coal Creek Station) used some of the data in their mass balance or emission factor calculations. In some cases, the differences between the round-robin results and the plant results were quite large, as shown in Tables 4-5 and 4-6. These differences may stem in part from the fact that the round-robin and plant samples were not necessarily equivalent splits representing the same time period. Some of the differences shown in Tables 4-5 and 4-6 may also reflect analytical uncertainty.

EER repeated the Cardinal Station crushed coal analyses using a different laboratory than used for the round-robin samples. Round-robin results from the original laboratory were very inconsistent with both EER's analytical results from field samples as well as the other contractor's round-robin results. The parameters with values that changed significantly as a result of the repeated work were fixed carbon, volatile matter, ultimate results, $\mathrm{Cl}, \mathrm{F}$, and all trace element determinations except Cd. EER used the second set of analytical results in its calculations, and these values are reported in Tables 4-3 and 4-4. The round-robin data in Tables 4-7 to 4-10 and Figures 4-1 to 4-3 represent work performed by the original laboratory.

Battelle substituted some round-robin results $(\mathrm{Sb}, \mathrm{As}, \mathrm{Ba}, \mathrm{B}, \mathrm{Cd}, \mathrm{Pb}, \mathrm{Hg}, \mathrm{Mo}, \mathrm{Ni}$, and $\mathrm{Se}$ ) for its plant data for Coal Creek Station in mass balance and emission factor calculations. The elements that were substituted either had nondetect values in the plant data or had relative differences of $>30 \%$ between the plant values and the roundrobin values. Some of the difficulties in analyzing Coal Creek Station's coal probably stemmed from its low rank (lignite), as discussed in Section 4.1.4.

For the report on Niles Station, Battelle used round-robin data for $\mathrm{Cd}, \mathrm{Mo}, \mathrm{Se}$, and $\mathrm{Sb}$ for mass balance and emission factor calculations. $\mathrm{Cd}$, Mo, and Se were reported as nondetects in the plant feed coal data. The round-robin value for $\mathrm{Sb}$ was used because Battelle's plant value differed by $43 \%$ from the round-robin result. 
Table 4-3. Plant Results for Each Coal: Dry Coal Proximate-Ultimate and Major Element Analyses (in weight percent [except Btu/lb] \pm percent relative standard deviation)

\begin{tabular}{|c|c|c|c|c|c|c|c|c|c|}
\hline \multicolumn{2}{|c|}{ Parameter Coal Creek } & Niles & SNOX & Cardinal & Yates & Bailly & Springerville & Baldwin & Boswell \\
\hline \multicolumn{10}{|c|}{ Proximate-Ultimate Analyses, wt $\%$} \\
\hline Ash & $17 \pm 8.6$ & $12 \pm 1.2$ & $12 \pm 0.83$ & $11 \pm 2.7$ & $11 \pm 4.0$ & $12 \pm 2.7$ & $24 \pm 1.5$ & $12 \pm 3.3$ & $11 \pm 3.7$ \\
\hline c & $59 \pm 2.7$ & $72 \pm 0.25$ & $72 \pm 0.47$ & $72 \pm 0.58$ & $72 \pm 0.23$ & $69 \pm 0.83$ & $59 \pm 0.72$ & $70 \pm 0.8$ & $68 \pm 0.3$ \\
\hline$H$ & $3.9 \pm 7.4$ & $4.8 \pm 0.83$ & $4.8 \pm 1.0$ & $5.0 \pm 0.65$ & $4.8 \pm 0.09$ & $5.0 \pm 2.2$ & $4.6 \pm 1.2$ & $4.9 \pm 0.9$ & $4.7 \pm 0.6$ \\
\hline N & $0.88 \pm 5.7$ & $1.5 \pm 2.0$ & $1.5 \pm 1.3$ & $1.5 \pm 4.1$ & $1.5 \pm 2.9$ & $1.2 \pm 0.94$ & $1.1 \pm 3.9$ & $1.3 \pm 3.1$ & $0.96 \pm 4.4$ \\
\hline s & $1.0 \pm 13$ & $2.8 \pm 2.1$ & $2.6 \pm 2.7$ & $3.0 \pm 2.0$ & $2.7 \pm 3.3$ & $3.5 \pm 2.5$ & $0.68 \pm 6.5$ & $3.4 \pm 2.3$ & $0.93 \pm 4.5$ \\
\hline $\mathrm{Cl}$ & $N R^{a}$ & NR & NR & $0.095 \pm 3.4$ & $0.14 \pm 2.0$ & $0.11 \pm 0.00$ & $0.04 \pm 7.8$ & $0.08 \pm 12$ & $0.0038 \pm 6.4$ \\
\hline Btu/lb & $9970 \pm 2.6$ & $12900 \pm 0.50$ & $13000 \pm 0.24$ & $13100 \pm 0.70$ & $12700 \pm 0.16$ & $12400 \pm 0.05$ & $10400 \pm 0.69$ & $12500 \pm 0.5$ & $11700 \pm 0.6$ \\
\hline \multicolumn{10}{|c|}{ Major Elements, wt\% } \\
\hline $\mathrm{Al}_{2} \mathrm{O}_{3}$ & $3.7 \pm 12$ & $5.7 \pm 1.5$ & $5.5 \pm 2.6$ & $22 \pm 0.95$ & $5.5 \pm 3.0$ & $4.5 \pm 5.7$ & $13 \pm 42$ & $19 \pm 2.5$ & $19 \pm 3.3$ \\
\hline $\mathrm{CaO}$ & NR & NR & NR & $1.2 \pm 5.4$ & $0.29 \pm 20$ & $0.50 \pm 21$ & $0.91 \pm 54$ & $5.7 \pm 6.8$ & $12 \pm 4.5$ \\
\hline $\mathrm{Fe}_{2} \mathrm{O}_{3}$ & NR & NR & NR & $26 \pm 1.2$ & $3.3 \pm 3.0$ & $4.3 \pm 9.0$ & $2.4 \pm 46$ & $17 \pm 10$ & $6.4 \pm 8.9$ \\
\hline $\mathrm{MgO}$ & NR & NR & NR & $0.62 \pm 6.5$ & $0.10 \pm 9.4$ & $0.13 \pm 9.4$ & $0.33 \pm 40$ & $1.1 \pm 5.6$ & $4.0 \pm 1.9$ \\
\hline $\mathrm{P}_{2} \mathrm{O}_{5}$ & NR & NR & NR & $0.11 \pm 2.7$ & $0.04 \pm 6.0$ & $0.06 \pm 23$ & $0.029 \pm 31$ & $0.26 \pm 17$ & $0.28 \pm 8.8$ \\
\hline $\mathrm{K}_{2} \mathrm{O}$ & $0.47 \pm 20$ & $0.53 \pm 2.8$ & $0.51 \pm 5.8$ & $1.9 \pm 0.31$ & $0.80 \pm 6.9$ & NR & NR & $2.2 \pm 3.5$ & $0.51 \pm 22$ \\
\hline $\mathrm{SiO}_{2}$ & $5.4 \pm 14$ & $5.6 \pm 1.3$ & $5.6 \pm 1.3$ & $46 \pm 0.52$ & NR & NR & NR & $50 \pm 2.4$ & $44 \pm 2.8$ \\
\hline $\mathrm{Na}_{2} \mathrm{O}$ & $0.36 \pm 29$ & $0.14 \pm 0.00$ & $0.14 \pm 33$ & $0.65 \pm 13$ & $0.26 \pm 4.1$ & NR & NR & $0.9 \pm 12$ & $0.27 \pm 13$ \\
\hline $\mathrm{SO}_{3}$ & NR & NR & NR & $1.4 \pm 5.4$ & NR & NR & NR & $3.1 \pm 11$ & $11 \pm 5.0$ \\
\hline $\mathrm{TiO}_{2}$ & $0.80 \pm 11$ & $0.14 \pm 0.00$ & $0.14 \pm 7.6$ & $1.0 \pm 0.50$ & $0.15 \pm 6.0$ & $0.11 \pm 4.1$ & $0.25 \pm 40$ & $0.88 \pm 2.4$ & $0.77 \pm 2.9$ \\
\hline
\end{tabular}

${ }^{a}$ Not reported.

Table 4-4. Plant Results for Each Coal: Dry Coal Trace Element Analyses $(\mu \mathrm{g} / \mathrm{g} \pm$ percent relative standard deviation)

\begin{tabular}{|c|c|c|c|c|c|c|c|c|c|}
\hline Parameter & Coal Creek & Niles & SNOX & Cardinal & Yates & Bailly & Springerville & Baldwin & Boswell \\
\hline Sb & $N D^{a}<45$ & $1.2 \pm 32$ & $0.96 \pm 22$ & $0.67 \pm 12$ & $0.61 \pm 8.2$ & $0.71 \pm 7.8$ & $1.6 \pm 130$ & $0.57 \pm 8.4$ & $0.50 \pm 7.6$ \\
\hline As & $12 \pm 8.5$ & $35 \pm 4.6$ & $36 \pm 38$ & $8.9 \pm 20$ & $2.3 \pm 19$ & $3.1 \pm 38$ & $3.1 \pm 7.2$ & $3.1 \pm 30$ & $1.8 \pm 15$ \\
\hline $\mathrm{Ba}$ & $400 \pm 13$ & $59 \pm 1.8$ & $67 \pm 37$ & $31 \pm 4.6$ & $80 \pm 20$ & $47 \pm 5.0$ & $340 \pm 15$ & $60 \pm 20$ & $420 \pm 11$ \\
\hline $\mathrm{Be}$ & $0.82 \pm 9.0$ & $2.0 \pm 17$ & $2.4 \pm 11$ & $1.1 \pm 2.6$ & $1.1 \pm 0.00$ & $1.9 \pm 17$ & $1.2 \pm 8.0$ & $1.2 \pm 11$ & $0.24 \pm 13$ \\
\hline B & $170 \pm 4.1$ & $77 \pm 6.3$ & $56 \pm 55$ & $82 \pm 16$ & $100 \pm 0.00$ & $220 \pm 7.7$ & $110 \pm 7.0$ & $185 \pm 4.0$ & $300 \pm 15$ \\
\hline Cd & ND $<1.1$ & ND $<0.32$ & ND $<0.32$ & $0.77 \pm 140$ & $0.30 \pm 0.00$ & $3.0 \pm 32$ & ND $<0.53$ & $0.63 \pm 21$ & $0.08 \pm 14$ \\
\hline $\mathrm{Cr}$ & $10 \pm 20$ & $17 \pm 6.3$ & $16 \pm 10$ & $15 \pm 5.0$ & $25 \pm 3.7$ & $47 \pm 30$ & $9.8 \pm 2.9$ & $29 \pm 14$ & $4.2 \pm 6.8$ \\
\hline Co & $2.7 \pm 21$ & $6.7 \pm 24$ & $5.7 \pm 8.5$ & $4.5 \pm 11$ & $3.5 \pm 17$ & $2.8 \pm 10$ & $3.7 \pm 11$ & $3.6 \pm 1.8$ & $1.4 \pm 5.8$ \\
\hline $\mathrm{Cu}$ & $8.5 \pm 12$ & $16 \pm 3.9$ & $15 \pm 11$ & $7.0 \pm 0.00$ & $36 \pm 54$ & $11 \pm 9.6$ & $13 \pm 4.8$ & $9.7 \pm 3.4$ & $7.0 \pm 0.7$ \\
\hline $\mathrm{Pb}$ & $8.9 \pm 4.4$ & $14 \pm 13$ & $14 \pm 13$ & $9.3 \pm 11$ & $8.0 \pm 9.8$ & $8.5 \pm 15$ & $N D<5.3$ & $11 \pm 32$ & $5.2 \pm 10$ \\
\hline $\mathrm{Mn}$ & $120 \pm 8.1$ & $27 \pm 6.0$ & $29 \pm 17$ & $18 \pm 9.7$ & $23 \pm 4.4$ & $32 \pm 1.1$ & $86 \pm 12$ & $47 \pm 4.7$ & $140 \pm 8.0$ \\
\hline $\mathrm{Hg}$ & $0.10 \pm 13$ & $0.22 \pm 25$ & $0.28 \pm 31$ & $0.10 \pm 13$ & $0.077 \pm 12$ & $0.11 \pm 20$ & $0.039 \pm 11$ & $0.07 \pm 16$ & $0.07 \pm 11$ \\
\hline Mo & $2.8 \pm 27$ & $N D<3.2$ & $N D<3.2$ & $3.0 \pm 0.00$ & $22 \pm 8.6$ & $8.1 \pm 49$ & $2.3 \pm 51$ & $7.3 \pm 11$ & $5.5 \pm 24$ \\
\hline $\mathrm{Ni}$ & $3.2 \pm 50$ & $19 \pm 18$ & $16 \pm 8.0$ & $13 \pm 4.4$ & $30 \pm 6.7$ & $26 \pm 43$ & $6.1 \pm 2.3$ & $18 \pm 13$ & $2.8 \pm 3.1$ \\
\hline Se & $N D<1.6$ & $N D<0.64$ & $0.96 \pm 73$ & $2.0 \pm 14$ & $2.3 \pm 19$ & $1.5 \pm 63$ & $0.91 \pm 72$ & $3.7 \pm 10$ & $0.88 \pm 9.6$ \\
\hline$v$ & $18 \pm 17$ & $30 \pm 6.1$ & $28 \pm 10$ & $26 \pm 5.5$ & $39 \pm 0.96$ & $53 \pm 17$ & $28 \pm 1.1$ & $34 \pm 6.2$ & $8.4 \pm 7.2$ \\
\hline $\mathrm{F}$ & $\mathrm{NR}^{\mathrm{b}}$ & NR & NR & $56 \pm 5.2$ & $100 \pm 0.00$ & $110 \pm 1.1$ & $78 \pm 19$ & $114 \pm 9.1$ & $38 \pm 22$ \\
\hline
\end{tabular}

Nondetects.

bot reported. 
Table 4-5. Percent Difference Between Plant and Round-Robin Results for Proximate-Ultimate and Major Element Analyses

\begin{tabular}{|c|c|c|c|c|c|c|c|c|c|}
\hline Parameter & Coal Creek & Niles & SNOX & Cardinal & Yates & Bailly & Springerville & Baldwin & Boswell \\
\hline \multicolumn{10}{|c|}{ Proximate-Ultimate Analyses } \\
\hline Ash & 0 & -7.7 & -7.7 & -8.3 & 0 & -7.7 & +14 & 0 & -8.3 \\
\hline c & 0 & +2.9 & +2.9 & 0 & +1.4 & -1.4 & -3.3 & 0 & 0 \\
\hline H & -13 & -2.0 & -2.0 & 0 & -5.9 & +4.2 & -4.2 & 0 & -2.1 \\
\hline$N$ & -1.1 & +7.1 & +7.1 & +7.1 & +7.1 & -7.7 & 0 & 0 & -4.0 \\
\hline $\mathbf{s}$ & -9.1 & -6.7 & -13 & -9.1 & -6.9 & 0 & +4.6 & 0 & -22.5 \\
\hline $\mathrm{Cl}$ & $N^{a}$ & NR & NR & +12 & +17 & +49 & +2.6 & +25 & -87 \\
\hline Btu/lb & +3.9 & +3.2 & +4.0 & +1.6 & +0.79 & +1.6 & -1.9 & +2.5 & +2.6 \\
\hline \multicolumn{10}{|c|}{ Major Elements } \\
\hline $\mathrm{Al}_{2} \mathrm{O}_{3}$ & -66 & -75 & -76 & +4.8 & -75 & -76 & -38 & +12 & +12 \\
\hline $\mathrm{CaO}$ & NR & NR & NR & +20 & -88 & -82 & -72 & +39 & +54 \\
\hline $\mathrm{Fe}_{2} \mathrm{O}_{3}$ & NR & NR & NR & +4.0 & -79 & -71 & -47 & +6.3 & +4.9 \\
\hline $\mathrm{MgO}$ & NR & NR & NR & +3.3 & -87 & -84 & -65 & +43 & +54 \\
\hline $\mathrm{P}_{2} \mathrm{O}_{5}$ & NR & NR & NR & -31 & -85 & -83 & -28 & -10 & -24 \\
\hline $\mathrm{K}_{2} \mathrm{O}$ & -66 & -76 & -77 & +5.6 & -68 & NR & NR & +10 & 0 \\
\hline $\mathrm{SiO}_{2}$ & -87 & -88 & -88 & +2.2 & NR & NR & NR & +11 & +4.8 \\
\hline $\mathrm{Na}_{2} \mathrm{O}$ & -57 & -53 & -53 & +51 & -69 & NR & NR & -1.1 & -6.8 \\
\hline $\mathrm{SO}_{3}$ & NR & NR & NR & 0 & NR & NR & NR & -33 & 0 \\
\hline $\mathrm{TiO}_{2}$ & +70 & -88 & -88 & +1.0 & -85 & -89 & -75 & -1.1 & -12 \\
\hline
\end{tabular}

alant value not reported.

Table 4-6. Percent Difference Between Plant and Round-Robin Results for Trace Element Analyses

\begin{tabular}{lccccccccc}
\hline Parameter & Coal Creek & Niles & SNOX & Cardinal & Yates & Bailly & Springerville & Baldwin & Boswell \\
\hline $\mathrm{Sb}$ & $<+5900$ & -43 & -54 & +4.7 & -1.6 & -10 & -5.9 & +16 & +6.4 \\
$\mathrm{As}$ & +58 & +35 & +39 & -5.3 & -34 & +15 & +82 & +29 & +50 \\
$\mathrm{Ba}$ & -30 & -22 & -12 & 0 & +63 & -4.1 & +9.7 & +28 & +13.5 \\
$\mathrm{Be}$ & +14 & -17 & 0 & -15 & -21 & +19 & -7.7 & -8.3 & -43 \\
$\mathrm{~B}$ & +31 & +8.5 & -21 & +14 & -41 & +4.8 & +43 & -20 & +261 \\
$\mathrm{Cd}$ & $<+1300$ & $<+280$ & $<+280$ & +600 & -41 & +200 & $<-5.4$ & +8.6 & +33 \\
$\mathrm{Cr}$ & +24 & -15 & -20 & -6.3 & +19 & +34 & +2.1 & +3.5 & -4.5 \\
$\mathrm{Co}$ & +29 & -4.3 & -19 & -18 & -21 & -22 & -9.8 & -7.8 & +63 \\
$\mathrm{Cu}$ & -8.6 & -24 & -29 & -18 & +180 & 0 & -13 & -12 & -26 \\
$\mathrm{~Pb}$ & +170 & 0 & 0 & +55 & -5.9 & -35 & $<-40$ & +21 & 0 \\
$\mathrm{Mn}$ & 0 & 0 & +7.4 & 0 & -21 & -5.9 & +12 & +15 & -6.7 \\
$\mathrm{Hg}$ & -33 & -15 & +7.7 & -23 & -9.4 & 0 & -51 & -30 & -17 \\
$\mathrm{Mo}$ & -30 & $<-29$ & $<-29$ & +58 & +280 & +2.5 & +9.5 & -12 & -30 \\
$\mathrm{Ni}$ & -56 & -32 & -43 & -7.1 & +67 & +37 & -10 & 0 & -45 \\
$\mathrm{Se}$ & $<+100$ & $<-75$ & -63 & +5.3 & +4.6 & -53 & -24 & +28 & +4.8 \\
$\mathrm{~V}$ & +5.9 & -12 & -18 & -16 & 0 & +15 & +7.7 & -8.1 & -11 \\
$\mathrm{~F}$ & $\mathrm{NR}$ & $\mathrm{NR}$ & $\mathrm{NR}$ & -3.5 & +25 & 0 & -2.5 & +18 & -14 \\
\hline
\end{tabular}

" $<$ " Plant data reported as ND.

- Plant value reported as fluoride. 
Table 4-7. Round-Robin Results for Each Coal: Dry Coal Proximate-Ultimate and Major Element Analyses (in weight percent [except Btu/lb] \pm percent relative standard deviation)

\begin{tabular}{|c|c|c|c|c|c|c|c|c|}
\hline Parameter & Coal Creek & Niles/SNOX & Cardinal & Yates & Bailly & Springerville & Baldwin & Boswell \\
\hline \multicolumn{9}{|c|}{ Proximate-Ultimate, wt $\%$} \\
\hline Ash & $17 \pm 2.7$ & $13 \pm 0.68$ & $12 \pm 0.56$ & $11 \pm 0.72$ & $13 \pm 0.66$ & $21 \pm 0.66$ & $12 \pm 1.5$ & $12 \pm 1.68$ \\
\hline C & $59 \pm 2.2$ & $70 \pm 2.1$ & $72 \pm 0.82$ & $71 \pm 2.8$ & $70 \pm 2.7$ & $61 \pm 1.9$ & $70 \pm 0.85$ & $68 \pm 2.0$ \\
\hline$H$ & $4.5 \pm 24$ & $4.9 \pm 4.5$ & $5.0 \pm 4.7$ & $5.1 \pm 11$ & $4.8 \pm 4.0$ & $4.8 \pm 7.7$ & $4.9 \pm 5.8$ & $4.8 \pm 14$ \\
\hline$N$ & $0.89 \pm 20$ & $1.4 \pm 3.2$ & $1.4 \pm 3.2$ & $1.4 \pm 4.1$ & $1.3 \pm 3.7$ & $1.1 \pm 4.9$ & $1.3 \pm 5.4$ & $1.0 \pm 8.6$ \\
\hline$s$ & $1.1 \pm 3.5$ & $3.0 \pm 2.4$ & $3.3 \pm 4.0$ & $2.9 \pm 4.8$ & $3.5 \pm 4.5$ & $0.65 \pm 16$ & $3.4 \pm 6.8$ & $1.2 \pm 53$ \\
\hline $\mathrm{Cl}$ & $0.040 \pm 25$ & $0.14 \pm 61$ & $0.085 \pm 25$ & $0.12 \pm 29$ & $0.074 \pm 31$ & $0.039 \pm 58$ & $0.064 \pm 23$ & $0.03 \pm 46$ \\
\hline Btu/lb & $9600 \pm 6.4$ & $12500 \pm 3.6$ & $12900 \pm 1.6$ & $12600 \pm 3.5$ & $12200 \pm 3.4$ & $10600 \pm 4.4$ & $12200 \pm 4.9$ & $11400 \pm 6.8$ \\
\hline \multicolumn{9}{|c|}{ Major Elements, wt\% } \\
\hline $\mathrm{Al}_{2} \mathrm{O}_{3}$ & $11 \pm 35$ & $23 \pm 4.7$ & $21 \pm 4.2$ & $22 \pm 7.2$ & $19 \pm 4.6$ & $21 \pm 11$ & $17 \pm 17$ & $17 \pm 26$ \\
\hline $\mathrm{CaO}$ & $11 \pm 64$ & $1.5 \pm 22$ & $1.0 \pm 27$ & $2.5 \pm 31$ & $2.7 \pm 39$ & $3.3 \pm 38$ & $4.1 \pm 51$ & $7.8 \pm 62$ \\
\hline $\mathrm{Fe}_{2} \mathrm{O}_{3}$ & $6.1 \pm 16$ & $21 \pm 3.4$ & $25 \pm 5.7$ & $16 \pm 11$ & $15 \pm 37$ & $4.5 \pm 7.4$ & $16 \pm 7.7$ & $6.1 \pm 15$ \\
\hline MgO & $3.0 \pm 66$ & $0.73 \pm 29$ & $0.60 \pm 31$ & $0.79 \pm 38$ & $0.82 \pm 43$ & $0.93 \pm 48$ & $0.77 \pm 44$ & $2.6 \pm 60$ \\
\hline $\mathrm{P}_{2} \mathrm{O}_{5}$ & $0.17 \pm 39$ & $0.58 \pm 34$ & $0.16 \pm 37$ & $0.26 \pm 31$ & $0.36 \pm 30$ & $0.04 \pm 38$ & $0.29 \pm 35$ & $0.37 \pm 39$ \\
\hline $\mathrm{K}_{2} \mathrm{O}$ & $1.4 \pm 23$ & $2.2 \pm 8.9$ & $1.8 \pm 7.8$ & $2.5 \pm 12$ & $2.2 \pm 7.4$ & $1.3 \pm 8.4$ & $2.0 \pm 13$ & $0.51 \pm 18$ \\
\hline $\mathrm{SiO}_{2}$ & $40 \pm 3.2$ & $46 \pm 3.1$ & $45 \pm 1.9$ & $52 \pm 11$ & $50 \pm 3.4$ & $59 \pm 3.3$ & $45 \pm 18$ & $42 \pm 3.3$ \\
\hline $\mathrm{Na}_{2} \mathrm{O}$ & $0.84 \pm 7.8$ & $0.30 \pm 26$ & $0.43 \pm 14$ & $0.84 \pm 9.6$ & $0.75 \pm 14$ & $0.23 \pm 26$ & $0.91 \pm 9.4$ & $0.29 \pm 12$ \\
\hline $\mathrm{SO}_{3}$ & $15 \pm 3.4$ & $1.7 \pm 8.0$ & $1.4 \pm 18$ & $2.6 \pm 13$ & $2.6 \pm 32$ & $3.7 \pm 4.2$ & $4.6 \pm 37$ & $11 \pm 29$ \\
\hline $\mathrm{TiO}_{2}$ & $0.47 \pm 5.7$ & $1.2 \pm 32$ & $0.99 \pm 5.6$ & $1.0 \pm 30$ & $0.99 \pm 26$ & $1.0 \pm 16$ & $0.89 \pm 8.8$ & $0.88 \pm 16$ \\
\hline
\end{tabular}

Table 4-8. Round-Robin Results for Each Coal: Dry Coal Trace Element Analyses ( $\mu \mathrm{g} / \mathrm{g} \pm$ percent relative standard deviation)

\begin{tabular}{lcccccccc}
\hline Parameter & Coal Creek & Niles/SNOX & Cardinal & Yates & Bailly & Springerville & Baldwin & Boswell \\
\hline $\mathrm{Sb}$ & $0.75 \pm 37$ & $2.1 \pm 12$ & $0.64 \pm 7.6$ & $0.62 \pm 25$ & $0.79 \pm 35$ & $1.7 \pm 44$ & $0.49 \pm 5.9$ & $0.47 \pm 5.0$ \\
$\mathrm{As}$ & $7.6 \pm 44$ & $26 \pm 39$ & $9.4 \pm 36$ & $3.5 \pm 29$ & $2.7 \pm 38$ & $1.7 \pm 39$ & $2.4 \pm 24$ & $1.2 \pm 40$ \\
$\mathrm{Ba}$ & $570 \pm 35$ & $76 \pm 27$ & $31 \pm 21$ & $49 \pm 14$ & $49 \pm 22$ & $310 \pm 45$ & $47 \pm 38$ & $370 \pm 53$ \\
$\mathrm{Be}$ & $0.72 \pm 17$ & $2.4 \pm 12$ & $1.3 \pm 16$ & $1.4 \pm 22$ & $1.6 \pm 16$ & $1.3 \pm 8.4$ & $1.3 \pm 11$ & $0.42 \pm 18$ \\
$\mathrm{~B}$ & $130 \pm 35$ & $71 \pm 18$ & $72 \pm 16$ & $170 \pm 22$ & $210 \pm 15$ & $77 \pm 17$ & $230 \pm 15$ & $83 \pm 34$ \\
$\mathrm{Cd}$ & $0.079 \pm 39$ & $0.085 \pm 39$ & $0.11 \pm 32$ & $0.51 \pm 58$ & $1.0 \pm 58$ & $0.56 \pm 140$ & $0.58 \pm 35$ & $0.06 \pm 63$ \\
$\mathrm{Cr}$ & $8.1 \pm 13$ & $20 \pm 21$ & $16 \pm 19$ & $21 \pm 14$ & $35 \pm 4.3$ & $9.6 \pm 14$ & $28 \pm 9.9$ & $4.4 \pm 19$ \\
$\mathrm{Co}$ & $2.1 \pm 46$ & $7.0 \pm 28$ & $5.5 \pm 31$ & $4.4 \pm 40$ & $3.6 \pm 21$ & $4.1 \pm 25$ & $3.9 \pm 29$ & $0.86 \pm 43$ \\
$\mathrm{Cu}$ & $9.3 \pm 30$ & $21 \pm 13$ & $8.5 \pm 20$ & $13 \pm 15$ & $11 \pm 18$ & $15 \pm 49$ & $11 \pm 18$ & $9.5 \pm 22$ \\
$\mathrm{~Pb}$ & $3.3 \pm 64$ & $14 \pm 34$ & $6.0 \pm 44$ & $8.5 \pm 39$ & $13 \pm 30$ & $8.9 \pm 23$ & $9.1 \pm 35$ & $5.2 \pm 27$ \\
$\mathrm{Mn}$ & $120 \pm 17$ & $27 \pm 11$ & $18 \pm 10$ & $29 \pm 13$ & $34 \pm 14$ & $77 \pm 20$ & $41 \pm 24$ & $150 \pm 19$ \\
$\mathrm{Hg}$ & $0.15 \pm 17$ & $0.26 \pm 20$ & $0.13 \pm 25$ & $0.085 \pm 26$ & $0.11 \pm 41$ & $0.080 \pm 9.1$ & $0.10 \pm 10$ & $0.084 \pm 17$ \\
$\mathrm{Mo}$ & $4.0 \pm 88$ & $4.5 \pm 46$ & $1.9 \pm 47$ & $5.8 \pm 48$ & $7.9 \pm 54$ & $2.1 \pm 97$ & $8.3 \pm 52$ & $7.9 \pm 55$ \\
$\mathrm{Ni}$ & $7.3 \pm 50$ & $28 \pm 38$ & $14 \pm 15$ & $18 \pm 25$ & $19 \pm 14$ & $6.8 \pm 18$ & $18 \pm 16$ & $5.1 \pm 90$ \\
$\mathrm{Se}$ & $0.80 \pm 34$ & $2.6 \pm 24$ & $1.9 \pm 26$ & $2.2 \pm 26$ & $3.2 \pm 38$ & $1.2 \pm 34$ & $2.9 \pm 21$ & $0.84 \pm 28$ \\
$\mathrm{~V}$ & $17 \pm 8.7$ & $34 \pm 14$ & $31 \pm 14$ & $39 \pm 15$ & $46 \pm 9.4$ & $26 \pm 6.1$ & $37 \pm 12$ & $9.4 \pm 9.3$ \\
$\mathrm{~F}$ & $57 \pm 7.7$ & $81 \pm 15$ & $58 \pm 7.8$ & $80 \pm 9.8$ & $110 \pm 15$ & $80 \pm 17$ & $97 \pm 16$ & $44 \pm 14$ \\
\hline
\end{tabular}




\subsubsection{Accuracy}

Analysis of NIST Standard SRM 1632b, a Pittsburgh seam coal, was used to evaluate the accuracy of each contractor's laboratory. For each laboratory, all detected results within $10 \%$ of the NIST value were considered accurate. Nondetect values were not used in calculating accuracy, although a significant number were reported, resulting largely from the different laboratory techniques chosen by individual contractors. NIST-certified or informational values for SRM $1632 \mathrm{~b}$ were available for all parameters in the round-robin study except $\mathrm{B}, \mathrm{Ba}, \mathrm{F}, \mathrm{P}$, and $\mathrm{Hg}$. Parameters without a NIST-certified or informational value were not included in the accuracy evaluation.

As shown in Table 4-9, the percentage of accurate trace element analyses ranged from $38 \%$ to $75 \%$. Nondetect results were reported for $\mathrm{Sb}, \mathrm{Cd}, \mathrm{Cu}, \mathrm{F}, \mathrm{Mo}, \mathrm{Ni}$, and $\mathrm{Se}$. The elements resulting in the most problematic trace element analyses were $\mathrm{Sb}$, As, $\mathrm{Cd}$, Mo, and Se. None of the laboratories utilized by the Phase I contractors were able to report trace element content accurately, i.e., within $10 \%$ of the NIST values more than $75 \%$ of the time. Overall, $57 \%$ of the reported trace element data for SRM $1632 \mathrm{~b}$ met this level of accuracy.

Proximate and ultimate results for SRM 1632b, with the exception of a single sulfur analysis, were within ASTM reproducibility limits of the NIST values for all laboratories except those originally utilized by EER where results exceeded ASTM limits for $\mathrm{H}, \mathrm{N}, \mathrm{S}$, and $\mathrm{Cl}$ and major elements $\mathrm{Si}, \mathrm{Fe}, \mathrm{Ca}, \mathrm{Mg}$, and $\mathrm{K}$ and heating value. Results from laboratories utilized by Weston and SRI were within ASTM reproducibility limits for all major elements. Laboratories utilized by Radian exceeded ASTM limits for

Table 4-9. Accuracy of Round-Robin Results for NIST SRM 1632b

\begin{tabular}{lccc}
\hline Contractor & $\begin{array}{c}\text { Results Above DL for } \\
\text { Trace Elements, \% }\end{array}$ & $\begin{array}{c}\text { Accurate Results for } \\
\text { Trace Elements, \% }\end{array}$ & $\begin{array}{c}\text { Accurate Results } \\
\text { for All Analyses, \% }\end{array}$ \\
\hline EER & 88 & 38 & 43 \\
Weston & 100 & 73 & 88 \\
Radian & 82 & 50 & 63 \\
Battelle & 80 & 75 & 80 \\
SRI & 100 & 48 & 78 \\
\hline
\end{tabular}


the major elements $\mathrm{Ca}, \mathrm{Mg}$, and $\mathrm{K}$. Laboratories utilized by Battelle reported results for $\mathrm{Al}$ and $\mathrm{K}$ beyond ASTM reproducibility limits and did not report any results for $\mathrm{Fe}, \mathrm{Ca}$, $\mathrm{Mg}, \mathrm{P}$, and $\mathrm{S}$.

\subsubsection{Reproducibility}

Interlaboratory reproducibility for all feed coals and all contractors was evaluated using percent relative standard deviation (PRSD). The average PRSD for all coals and all contractors was $\mathbf{2 7 . 9 \%}$. Average PRSD values for individual trace elements ranged from $11.0 \%$ for $V$ to $60.9 \%$ for Mo. The average PRSD, as well as the range of PRSDs for the entire suite of trace elements, is shown in Figure 4-1. For most of the coal samples, the PRSDs for $\mathrm{Cd}, \mathrm{Cu}$, and $\mathrm{Sb}$ are each based on results from only three laboratories because the results from laboratories utilized by Weston and Radian for these elements were either below detection limits or were not determined. The range of PRSDs was quite large; trace elements $\mathrm{Ba}, \mathrm{Cd}, \mathrm{Cu}, \mathrm{Hg}, \mathrm{Mo}, \mathrm{Ni}, \mathrm{Pb}$, and $\mathrm{Sb}$ all have PRSD ranges of over $30 \%$. For some samples, the range of reported values for $\mathrm{Mo}, \mathrm{Ni}$, and $\mathrm{Cd}$ was $52 \%, 76 \%$, and $110 \%$, respectively. The average PRSD for major elements was $21.7 \%$. P, Ca, and $\mathrm{Mg}$ each had PRSDs greater than $35 \%$. The only laboratories that did not exceed ASTM reproducibility limits for the major elements on SRM $1632 \mathrm{~b}$ were those contracted by Weston and SRI, for which the average PSRD for major elements was only $7 \%$.

Trace element analytical variability and as-determined coal heating values were examined in order to identify any effect of coal rank. Figure 4-2 shows average PRSD as a function of heating value for each feed coal in the round-robin study. Regression analysis of the data yields $r^{2}=0.95$, clearly indicating that as heating value (and thus coal rank) decreases, the analytical variability for trace elements increases. This trend is not unexpected, considering that many ASTM coal standards have rank-dependent precision statements. 


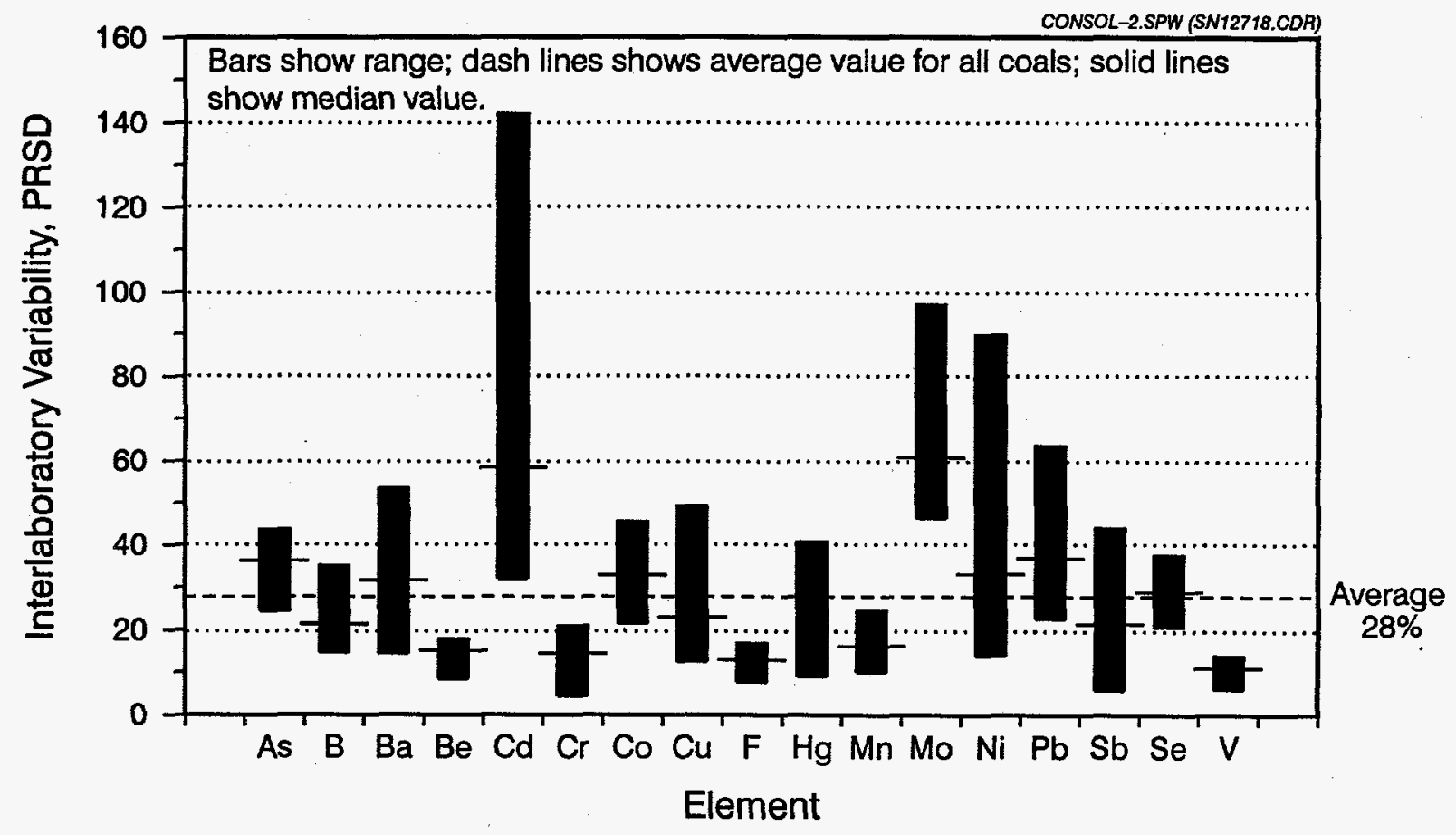

Figure 4-1. Interlaboratory Variability by Trace Element (from CONSOL INC roundrobin report).

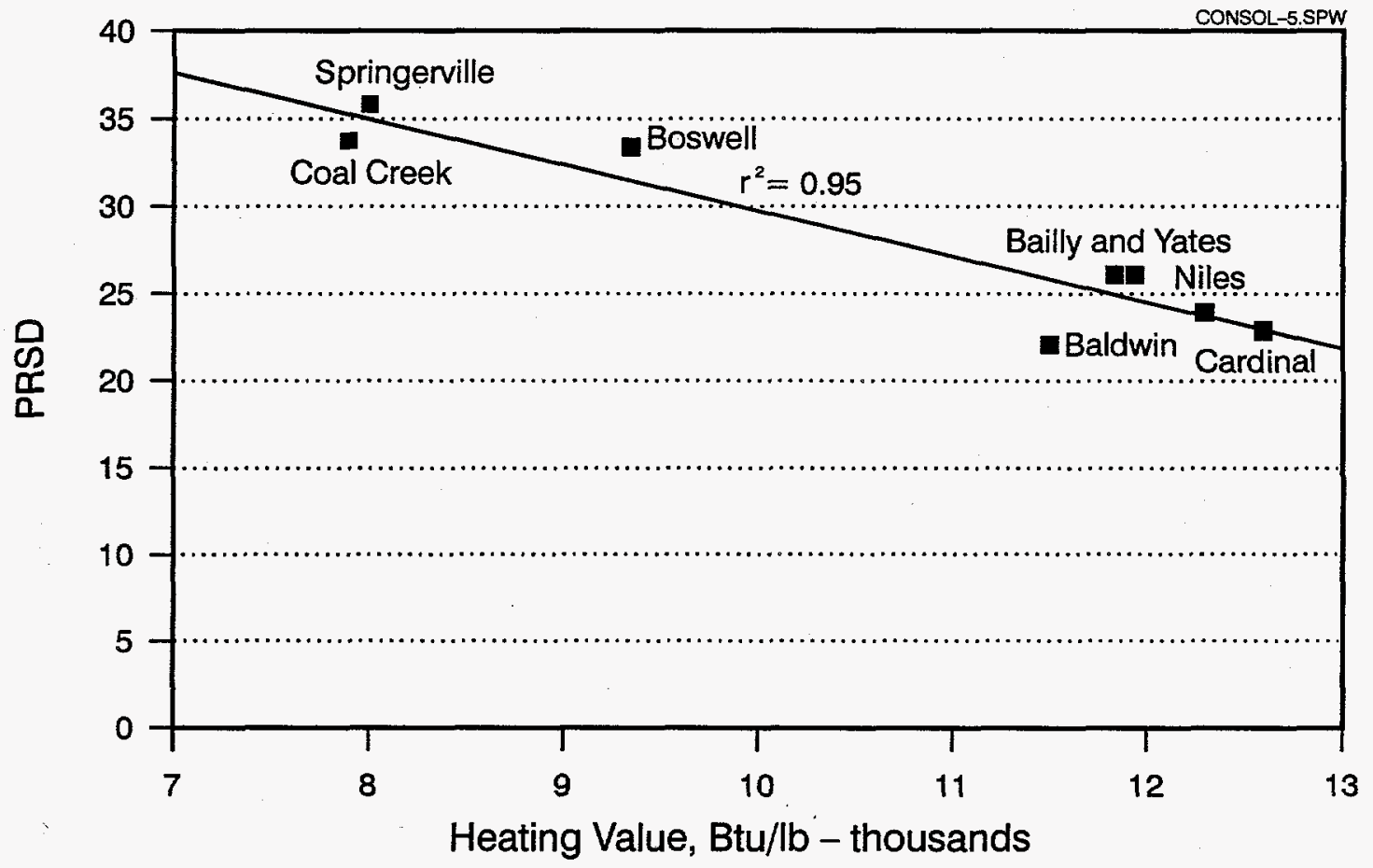

Figure 4-2. Correlation of Trace Element Analytical Variability with As-Determined Heating Value (from CONSOL INC round-robin report). 


\subsubsection{Repeatability}

Intralaboratory repeatability was calculated as the average percentage difference in a given laboratory's results on the eight duplicate samples. Each laboratory received 16 samples, consisting of duplicates of each of the eight feed coals, but the identity of the duplicates was unknown to the laboratories. Repeatability ranged from $7.8 \%$ for $\mathrm{Cr}$ to $32.5 \%$ for $\mathrm{Cd}$, as shown in Figure 4-3. The average repeatability for all trace elements was $14.6 \%$. Elements with lower interlaboratory reproducibility also tended to have lower intralaboratory repeatability. One exception was Mo, which had relatively low repeatability $(16.8 \%)$ but demonstrated the highest reproducibility $(60.7 \%)$, possibly indicating bias in the different methods used in its determination.

\subsubsection{Discussion: Comparison of Feed Coals}

The trace element content of the different feed coals used may be best compared on a per trillion Btu basis. Table 4-10 shows the trace elements present in each coal,

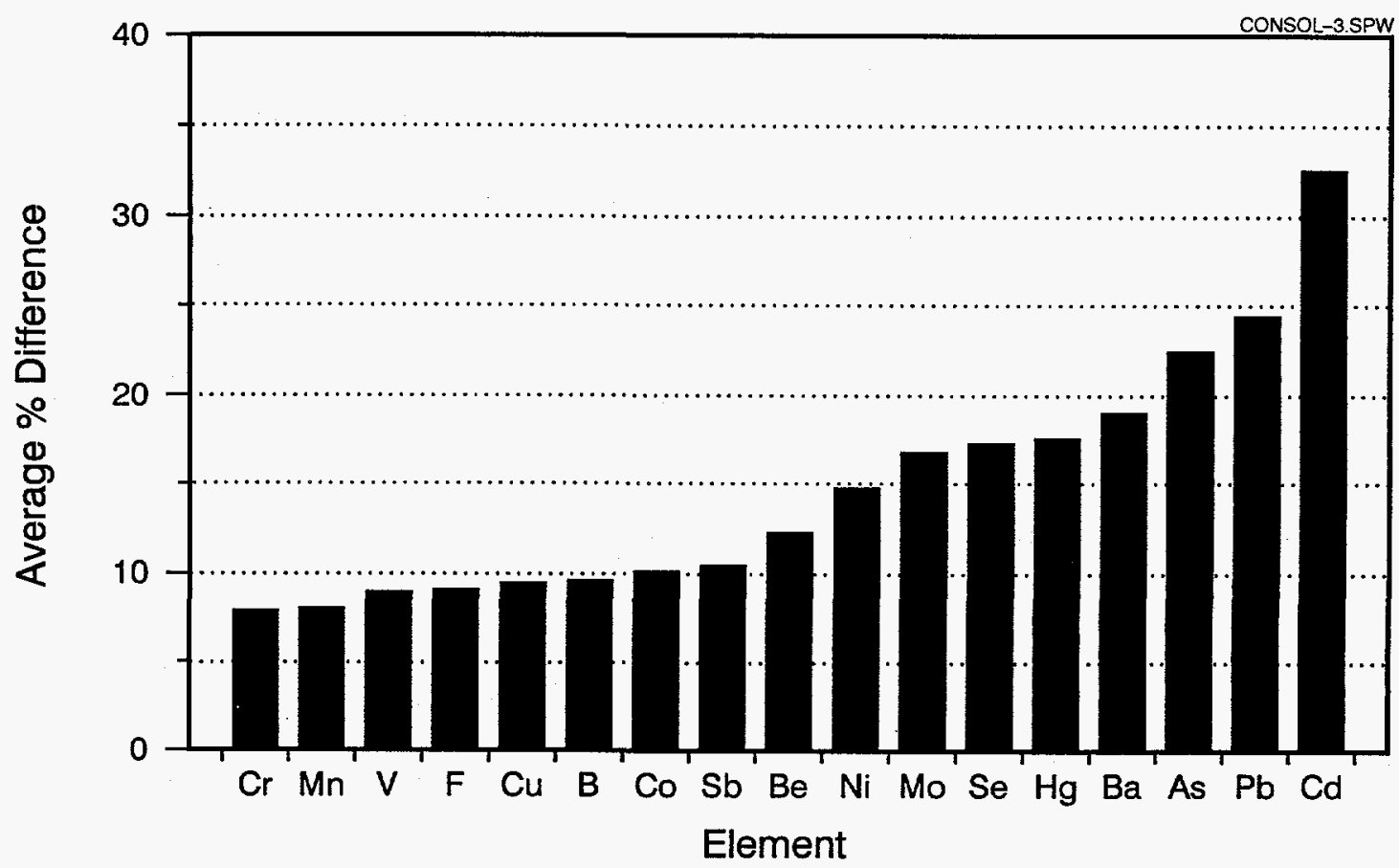

Figure 4-3. Average Intralaboratory Repeatability for Trace Elements (from CONSOL INC round-robin report). 
expressed as $\mathrm{lb} / 10^{12} \mathrm{Btu}$. Note that these are not emission factors, but rather represent the composition of the unburned coal. Coal trace element content was observed to vary within about 1 order of magnitude for each element.

For most of the trace elements shown in Table 4-10, stack emissions are likely to be low because these elements are contained in the fly ash, which can be effectively controlled using particulate control technology. The coal levels of the trace elements that exist in the vapor phase are the main concern. Of the eight feed coals tested, the Pennsylvania-Ohio bituminous blend used by Niles Station had the highest level of $\mathrm{Hg}$ $\left(21 \mathrm{lb} / 10^{12} \mathrm{Btu}\right)$, followed by the Falkirk lignite used by Coal Creek Station $\left(15 \mathrm{lb} / 10^{12} \mathrm{Btu}\right)$. The remaining feed coals had $\mathrm{Hg}$ concentrations in the range of 7 to $10 \mathrm{lb} / 10^{12}$ Btu.

Table 4-10. Coal Trace Element Content in $\mathrm{lb} / 10^{12}$ Btu (dry basis) Based on Average Round-Robin Results

\begin{tabular}{|c|c|c|c|c|c|c|c|c|}
\hline Parameter & Coal Creek & Niles/SNOX & Cardinal & Yates & Bailly & Springerville & Baldwin & Boswell \\
\hline Coal Type: & $\begin{array}{l}\text { Falkirk (ND) } \\
\text { Lignite }\end{array}$ & $\begin{array}{c}\text { PA-OH } \\
\text { Bituminous } \\
\text { Blend }\end{array}$ & $\begin{array}{c}\text { Pittsburgh } \\
\text { No. } 8 \\
\text { Bituminous }\end{array}$ & $\begin{array}{c}\text { IL Nos. } 5 / 6 \\
\text { Bituminous } \\
\text { Blend }\end{array}$ & $\begin{array}{l}\text { IL-IN Basin } \\
\text { Bituminous }\end{array}$ & $\begin{array}{c}\text { Lee Ranch } \\
\text { (NM) } \\
\text { Subbituminous }\end{array}$ & $\begin{array}{c}\text { IL } \\
\text { Bituminous } \\
\text { Blend }\end{array}$ & $\begin{array}{l}\text { Big Sky (PRB) } \\
\text { Subbituminous }\end{array}$ \\
\hline Btu/lb & 9600 & 12500 & 12900 & 12600 & 12200 & 10600 & 12200 & 11400 \\
\hline $\mathrm{Sb}$ & 78 & 170 & 50 & 48 & 65 & 160 & 40 & 41 \\
\hline As & 800 & 2100 & 730 & 270 & 230 & 160 & 200 & 110 \\
\hline $\mathrm{Ba}$ & 59000 & 6100 & 2400 & 3800 & 4000 & 29000 & 3900 & 33000 \\
\hline $\mathrm{Be}$ & 75 & 190 & 100 & 110 & 130 & 120 & 110 & 37 \\
\hline B & 13000 & 5700 & 5600 & 13000 & 17000 & 7200 & 19000 & 7400 \\
\hline $\mathrm{Cd}$ & 8.2 & 6.8 & 8.7 & 40 & 83 & 53 & 48 & 5.1 \\
\hline $\mathrm{Cr}$ & 840 & 1600 & 1300 & 1700 & 2900 & 900 & 2300 & 390 \\
\hline Co & 220 & 560 & 430 & 340 & 290 & 390 & 320 & 76 \\
\hline $\mathrm{Cu}$ & $\mathbf{9 7 0}$ & 1700 & 660 & 1000 & 930 & 1400 & 880 & 840 \\
\hline $\mathrm{Pb}$ & 350 & 1100 & 470 & 660 & 1100 & 830 & 750 & 460 \\
\hline Mn & 13000 & 2100 & 1400 & 2300 & 2800 & 7200 & 3400 & 13000 \\
\hline $\mathrm{Hg}$ & 15 & 21 & 10 & 6.6 & 8.9 & 7.5 & 8.3 & 7.4 \\
\hline Mo & 420 & 370 & 150 & 450 & 650 & 200 & 680 & 700 \\
\hline $\mathrm{Ni}$ & 760 & 2300 & 1100 & 1400 & 1500 & 640 & 1400 & 450 \\
\hline $\mathrm{Se}$ & 83 & 210 & 150 & 170 & 260 & 110 & 240 & 74 \\
\hline v & 1800 & 2700 & 2400 & 3000 & 3800 & 2500 & 3000 & 830 \\
\hline$F$ & 5900 & 6500 & 4500 & 6200 & 9200 & 7600 & 8000 & 3900 \\
\hline $\mathrm{Cl}$ & 42000 & 110000 & 66000 & 89000 & 61000 & 37000 & 52000 & 26000 \\
\hline
\end{tabular}


Similarly, As was highest in the Niles Station feed coal $\left(2100 \mathrm{lb} / 10^{12} \mathrm{Btu}\right)$ and in the Coal Creek Station feed coal $\left(800 \mathrm{lb} / 10^{12} \mathrm{Btu}\right)$. Overall, the Pennsylvania-Ohio bituminous blend used at Niles Station had the highest levels for the greatest number of trace elements (eight elements, including $\mathrm{Sb}, \mathrm{As}, \mathrm{Be}, \mathrm{Co}, \mathrm{Cu}, \mathrm{Pb}, \mathrm{Hg}$, and $\mathrm{Ni}$ ). The Illinois-Indiana Basin bituminous coal used at Bailly Station also had the highest levels for several trace elements (five, including $\mathrm{Cd}, \mathrm{Cr}, \mathrm{Se}, \mathrm{V}$, and $\mathrm{F}$ ). The PRB subbituminous coal used at Boswell Energy Center had the greatest number of low values (for nine trace elements, including $\mathrm{As}, \mathrm{Be}, \mathrm{Cd}, \mathrm{Cr}, \mathrm{Co}, \mathrm{Ni}, \mathrm{Se}, \mathrm{V}$, and $\mathrm{F}$ ). The Pittsburgh No. 8 bituminous coal used at Cardinal Station also had the lowest values for several trace elements (five, including $\mathrm{Ba}, \mathrm{B}, \mathrm{Cu}, \mathrm{Mn}$, and $\mathrm{Mo}$ ). Despite the differences in feed coal trace element content shown in Table 4-10, the emission control technology at each plant is likely to have a much greater influence on the actual levels of each element emitted from the stack.

\subsection{Trace Element Emission Factors}

Emission factor data were generated for 25 elements, including nine major elements and 16 minor or trace elements in the DOE Phase I study. The major elements included $\mathrm{Al}, \mathrm{Ca}, \mathrm{Fe}, \mathrm{Mn}, \mathrm{K}, \mathrm{Na}, \mathrm{Si}, \mathrm{Sr}$, and $\mathrm{Ti}$. The primary purpose for including a number of major elements in the study was to permit a better assessment of material balance results. Minor or trace elements included $\mathrm{Sb}, \mathrm{As}, \mathrm{Ba}, \mathrm{Be}, \mathrm{B}, \mathrm{Cd}, \mathrm{Cr}, \mathrm{Co}, \mathrm{Cu}$, $\mathrm{Pb}, \mathrm{Mn}, \mathrm{Hg}, \mathrm{Mo}, \mathrm{Ni}, \mathrm{Se}$, and $\mathrm{V}$. Eleven of the trace elements included in the DOE Phase I study are also found in the list of 189 HAPs identified in the CAAA of 1990. These are $\mathrm{Sb}, \mathrm{As}, \mathrm{Be}, \mathrm{Cd}, \mathrm{Cr}, \mathrm{Co}, \mathrm{Pb}, \mathrm{Mn}, \mathrm{Hg}, \mathrm{Ni}$, and $\mathrm{Se}$. Of the remaining four elements, $\mathrm{Ba}$ is regulated by the Resource Conservation and Recovery Act (RCRA), and $B$ and Mo are regulated by Irrigation Water Standards. Vanadium, although not specifically regulated as an element, is regulated based on its oxidation state. Vanadium pentoxide is a highly toxic regulated compound. The remainder of this section will focus on trace element data.

A discussion of trace element emissions from coal-fired combustion systems begins by classifying the various trace elements with respect to their degree of volatility. Trace element volatility plays a significant role in trace element partitioning in 
coal-fired systems and the degree of emissions control that can be expected from various technology options (ESP, FF, dry FGD, and wet FGD). Based on volatility within combustion systems, trace elements are usually associated with one of three classifications. Class I trace elements are the least volatile and are found to be equally distributed between bottom ash and fly ash. Class II trace elements can be somewhat volatile, resulting in bottom ash depletion and fly ash enrichment as a result of initial vaporization and subsequent condensation. Class III trace elements are the most volatile, found to exist entirely in the vapor phase and demonstrating no fly ash enrichment. Some degree of overlap exists between classifications for individual trace elements, depending on the fuel and combustion system design and operating conditions. Figure 4-4 illustrates volatility and/or partitioning classification for all major and minor or trace elements discussed in this report based on work by the EERC and others $(23-31)$.

Figure 4-5 is a graphical summary of the emission factors for 16 trace elements based on EPA Method 29 sampling data for each of the nine process configurations.
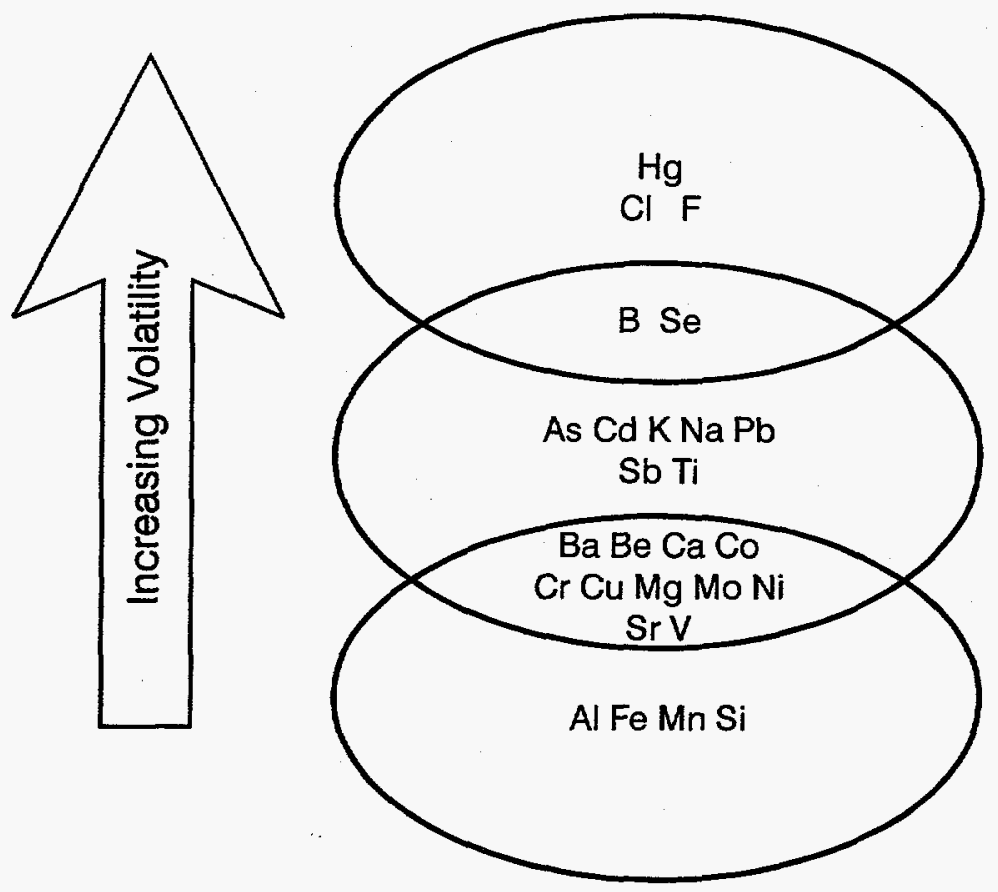

Class III Volatilized and Emitted Fully in the Vapor Phase Not Enriched in the Fly Ash

Class II

Enriched in the Fly Ash and Depleted in the Bottom Ash

Class 1 Equally Distributed Between the Bottom Ash and the Fly Ash

EERC GW11685.CDR

Figure 4-4. Classification of Selected Element Relative to Their Volatility and Partitioning in Power Plants $(26,30)$. 
Boron data were reported for only six sites. Data were reported for $\mathrm{Hg}$ for all nine process configurations. However, $\mathrm{Hg}$ data for two sites are based on sorbent trap data rather than EPA Method 29 data. The data are organized from left to right in descending order relative to the high end of the emission factor range for each trace element. Individual field sites were classified according to the type of emission control represented (ESP, FF, ESP-FGD, SDA-FF, and the SNOX process). Shaded symbols indicate data reported less than the detection limit for a given site and element. The data in Figure 4-5 show that the emission factor range for a given element was as small as 1 order of magnitude for $\mathrm{Mn}$ and $\mathrm{Hg}$ to 4 orders of magnitude for Se. Emission factors for the remaining 13 trace elements covered a range of 2 to 3 orders of magnitude. Based on median values, the data indicate that $B$ has the highest overall emission factor. Although $B$ is included in irrigation standards and can be toxic to vegetation, $B$ is not on the list of HAPs or RCRA elements. Therefore, B is not necessarily of significant interest to the original objective of the DOE Phase I study.

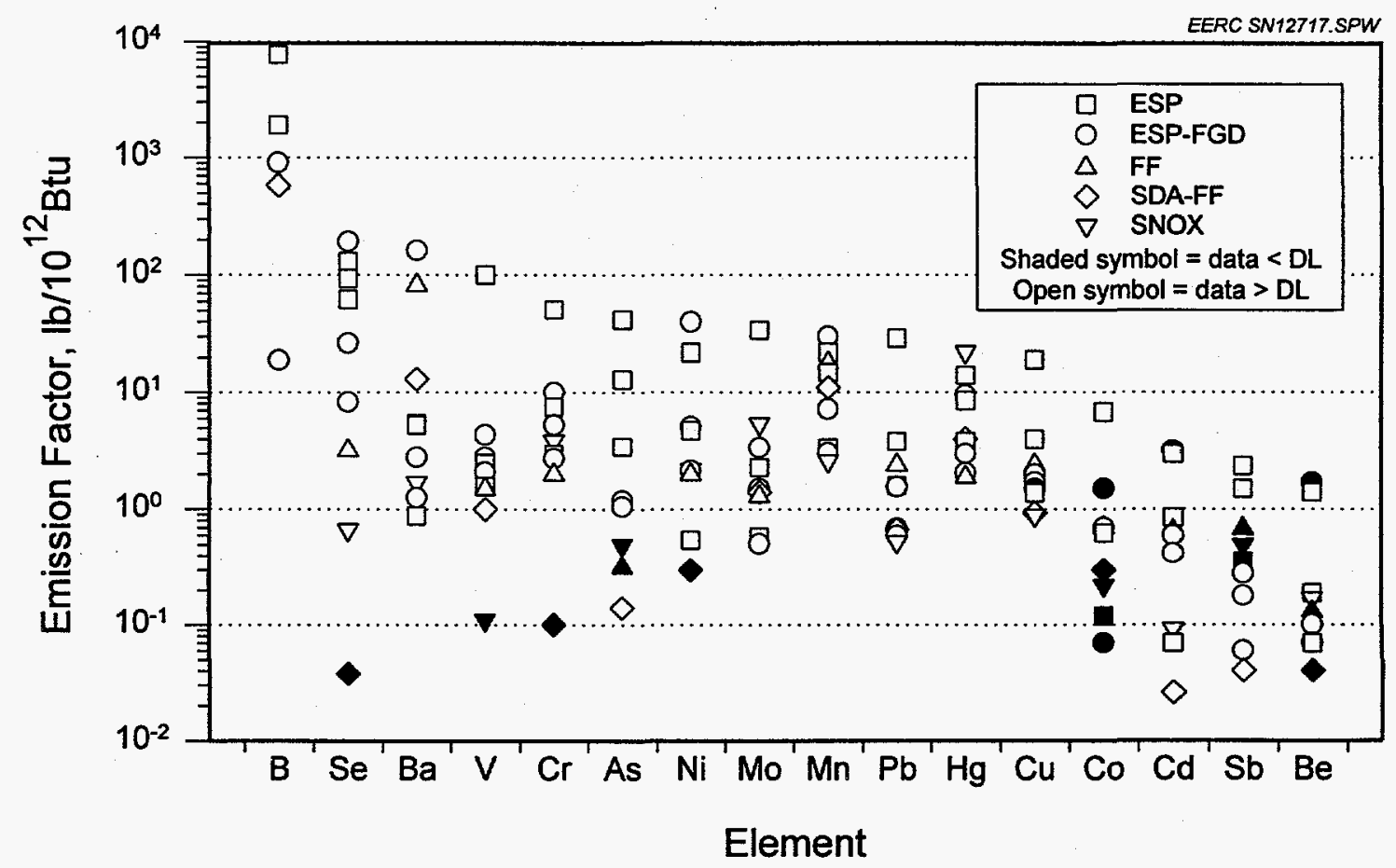

Figure 4-5. Emission Factors for 16 Trace Element at Each of Nine DOE Phase I Process Configurations. 
The trace element listed as a HAP and having the highest overall emissions was $\mathrm{Se}$, followed closely by $\mathrm{Mn}$. The lowest overall emissions were observed for $\mathrm{Sb}$ and $\mathrm{Be}$. Any interpretation of potential environmental impact should, however, be considered an oversimplification, as this data set does not contain information that is critical in determining health and environmental effects such as mobility, ambient concentration, and toxicity.

In this format, these data do not clearly indicate consistent differences in the relative performance of individual or combinations of emission control technology because of fuel variability, sampling/analytical precision, and other site differences (fuel type, boiler type, detailed process design, etc.). Because of the small size of the data set and the inherent differences between the individual field sites, it is not appropriate to develop hard conclusions concerning the performance of specific emission control technologies relative to individual trace elements. However, the data do imply that ESPs alone set an upper limit for nonvolatile trace element emissions. The SDA-FF system typically resulted in the lowest trace element emission level. With the exception of $\mathrm{Hg}$, the SNOX process also appeared to perform better than average for controlling trace element emissions.

Figure 4-6 presents a summary of DOE Phase I stack concentration data for 11 trace elements and nine process configurations and identifies the median concentrations. With the exception of $\mathrm{Hg}$, all of the stack concentration data are based on EPA Method 29 sampling. The trace element stack concentration data are compared to ambient air concentration data collected since 1980, obtained from an EPA report (31). The data set was limited to 11 trace elements to correspond with available ambient air concentration data. Figure 4-6 indicates that for nine (Sb, As, Be, Cd, Co, $\mathrm{Cr}, \mathrm{Hg}, \mathrm{Mn}$, and $\mathrm{Ni}$ ) of the 11 trace elements, the median stack concentrations are 2 to 3 orders of magnitude greater than the range of ambient air concentrations. For Se and $\mathrm{Pb}$, the differential was roughly 4 and 1 order of magnitude, respectively. Comparable stack and ambient air concentration data or ambient air quality standards are also presented for $\mathrm{HCl}, \mathrm{HF}, \mathrm{NO}_{x}$, and $\mathrm{SO}_{2}(32)$. In this case, a 3 to 4 order of magnitude differential exists between median stack concentration values and the respective ambient air concentration data or ambient air standard. These data imply, with the 


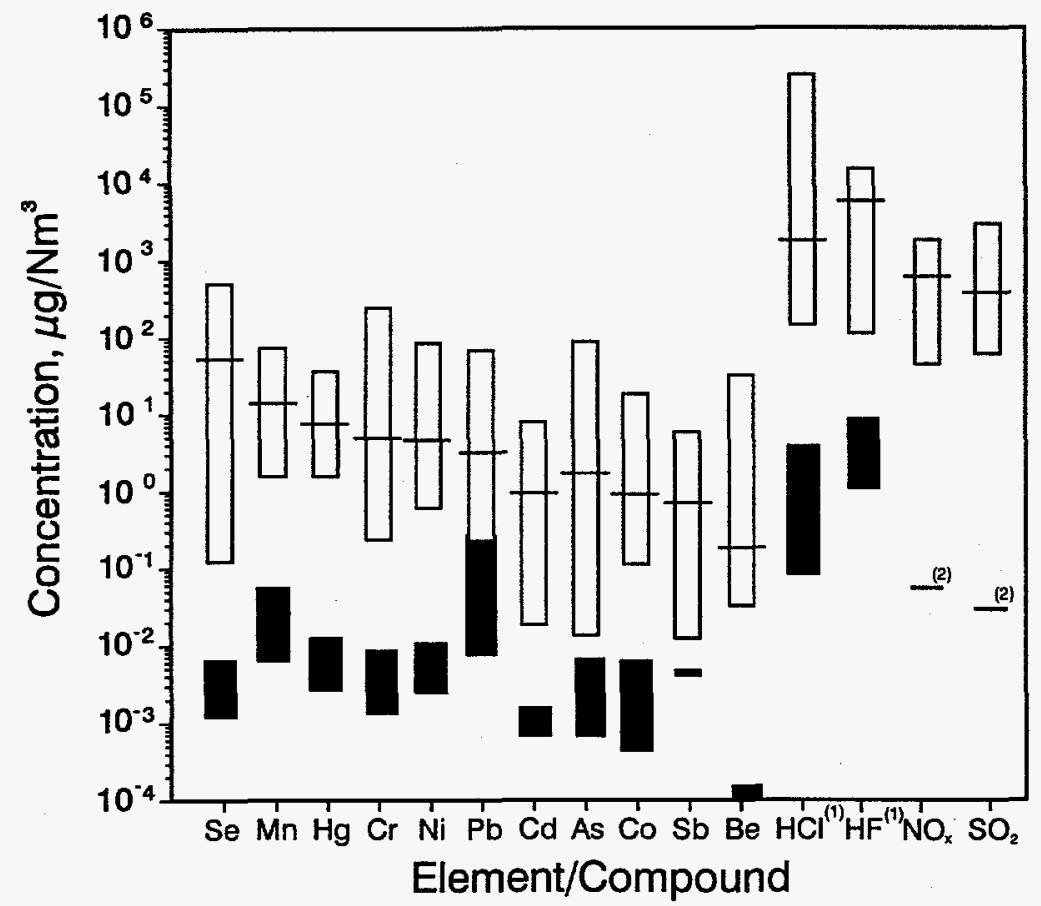

EERC SN12722A.CDR

Stack Concentration Range from DOE Phase I Field Tests

Ambient Air Concentration Range from Rural and Urban Areas Since 1980

- Median Concentration

Notes: Plant data median value calculation uses nondetect values equal to detection limits.

(1) Plant data are combined $\mathrm{HCl}+\mathrm{Cl}_{2}$ and $H F+F_{2}$. Ambient data are assumed to be combined also. Median not available for ambient air levels.

(2) Ambient air quality standard.

Figure 4-6. DOE Phase I Stack Concentration Data for 11 Trace Elements and Four Acid Gases Compared to EPA Ambient Air Data and Quality Standards.

exception of $\mathrm{Pb}$, that coal-fired power plants are possible contributors to ambient air trace element concentrations. The level or degree of contribution can only be determined as a function of extensive dilution and dispersion modeling, which is beyond the scope of this review effort.

Further discussion in this section of the report will focus on the criteria for assessing organic and inorganic data quality as well as emission factor and penetration data for $\mathrm{Hg}$, other volatile trace elements, and nonvolatile trace elements. Appendix A contains a series of tables that summarize the trace element data for the DOE Phase I field sampling activities, including data quality information relative to QA/OC criteria.

\subsubsection{Criteria for Assessing Organic and Inorganic Data Quality}

To determine the overall quality of a set of data from which a mean is calculated requires several concurrent steps. In order to perform an effective review of the DOE 
Phase I data, the EERC developed a tabular reporting format and data-flagging system as well as data evaluation criteria concerning blanks, spike recoveries, detection limits (DL), and lower limit of quantitation (LLQ as 10 times the DL). The intent of the reporting format, illustrated in Table 4-11, was to provide a format that could best serve all types of data users, including scientists, engineers, legislators, and regulators. This format has application to organic as well as inorganic data. Criteria for organic data are discussed in Section 4.3 of this report. The following is a short summary of the rationale behind the table.

In the second column, Total Stack Concentrations, all three or four data points should be reported. To assist the technical reader, the blank concentrations, spike, and audit recoveries should all be reported. The ambient air concentration, when available, should be reported to facilitate comparison with field values. Ambient air concentrations may be obtained from various sources $(33,34$, or on-site ambient air sampling). These concentrations are intended to provide information to make a cursory comparison between ambient air conditions and stack concentrations. They are not intended to be used as a blank or subtracted from the stack concentration. To assist the nontechnical reader, the data quality notation shows where quality controls passed or failed QA/OC criteria. Finally, the emission factor, the associated $95 \%$ confidence interva! (Cl), and emission control device performance are reported only for data that have 1) passed all QA/QC criteria and 2) been detected above the DL for at least three data points. To further avoid confusion and misuse of field test results, the data should be adequately flagged wherever they are cited outside of this report. Specific criteria for flagging data are suggested as follows:

1. Blank contained $>25 \%$ of given analyte.

2. Spike recovery was $<70 \%$ or $>125 \%$ for organics.

3. Spike recovery was $<70 \%$ or $>125 \%$ for inorganics.

4. Mean or calculated value was determined from three or four nondetects.

5. Mean or calculated value was determined from two nondetects and one or two detected values.

6. Mean or calculated value was determined from one nondetect and two or three detected values.

7. Less than three valid data points were available. 
Table 4-11. Proposed HAP Data Reporting Format

\section{Element/Compound}

\begin{tabular}{|c|c|c|c|c|c|c|c|c|c|c|c|c|c|c|c|c|}
\hline \multirow{2}{*}{$\begin{array}{l}\text { Station/ } \\
\text { Plant } \\
\text { Name }\end{array}$} & \multirow{2}{*}{$\begin{array}{c}\text { Total } \\
\text { Stack } \\
\text { Conc., } \\
\mu \mathrm{g} / \mathrm{Nm}^{3}\end{array}$} & \multirow{2}{*}{$\begin{array}{c}\text { Total } \\
\text { Mean } \\
\text { Conc., } \\
\mu \mathrm{g} / \mathrm{Nm}^{3}\end{array}$} & \multirow[b]{2}{*}{$\begin{array}{l}\text { Standard } \\
\text { Deviation }\end{array}$} & \multirow{2}{*}{$\begin{array}{c}\text { Blank } \\
\text { Conc. } \\
\text { Range, } \\
\mu \mathrm{g} / \mathrm{Nm}^{3}\end{array}$} & \multirow{2}{*}{$\begin{array}{c}\text { Ambient } \\
\text { Air } \\
\text { Standard } \\
\text { or } \\
\text { Sample, } \\
\mu \mathrm{g} / \mathrm{Nm}^{3}\end{array}$} & \multicolumn{3}{|c|}{$\begin{array}{l}\text { M29 Internal Spike } \\
\text { Recovery, \% }\end{array}$} & \multicolumn{3}{|c|}{$\begin{array}{l}\text { M29 Audit Spike } \\
\text { Recovery, } \%\end{array}$} & \multirow[b]{2}{*}{$\begin{array}{c}\text { Above } \\
\text { DL }\end{array}$} & \multirow[b]{2}{*}{$\begin{array}{l}\text { Above } \\
\text { LLO }\end{array}$} & \multirow{2}{*}{$\begin{array}{c}\text { Emission } \\
\text { Factor, } \\
\text { Ib/10 } 10^{12} \mathrm{Btu}\end{array}$} & \multirow{2}{*}{$\begin{array}{l}\text { Emission } \\
\text { Factor } \\
95 \% \mathrm{Cl}\end{array}$} & \multirow{2}{*}{$\begin{array}{c}\text { Control } \\
\text { Device } \\
\text { Removal, } \\
\%\end{array}$} \\
\hline & & & & & & Filter & $\begin{array}{l}\text { Front } \\
\text { Half }\end{array}$ & $\begin{array}{l}\text { Back } \\
\text { Half }\end{array}$ & Filter & $\begin{array}{l}\text { Front } \\
\text { Half }\end{array}$ & $\begin{array}{l}\text { Back } \\
\text { Half }\end{array}$ & & & & & \\
\hline $\begin{array}{l}\text { Station } \\
x\end{array}$ & $\begin{array}{l}256 \\
204 \\
192\end{array}$ & 217 & 34.0 & $\begin{array}{c}0.853 \\
N D<2.33 \\
P\end{array}$ & NA & $\begin{array}{c}93- \\
103 \\
P\end{array}$ & $\begin{array}{l}92.5 \\
P\end{array}$ & $\begin{array}{c}83 \\
P\end{array}$ & \multicolumn{3}{|c|}{ NA } & $P$ & $\mathrm{P}$ & 162 & 61 & ESP 99.3 \\
\hline
\end{tabular}

NOTE: The following criteria apply to the above table format (example entries are given for illustration).

- Blanks

- Blanks with less than $25 \%$ of the given analyte receive $P=$ pass.

- Blanks with greater than $25 \%$ of the given analyte receive $F=$ fail.

- Spike/audit

- Inorganic recoveries of $125 \%>x>70 \%$ receive $P=$ pass.

Inorganic recoveries of $x>125 \%$ or $x<70 \%$ receive $F=$ fail.

- Organic recoveries of $125 \%>x>70 \%$ receive $P=$ pass.

Organic recoveries of $x>125 \%$ or $x<70 \%$ receive $F=$ fail.

- Detection limit (DL)

- Values obtained above $D L$ receive $P=$ pass.

- Values obtained below $D L$ receive $F=$ fail.

- Lower limit of quantitation (LLQ)

- Values above the $L L Q$ defined as 10 times the $D L$ receive $P=$ pass.

- Values below the $L L O$ defined as 10 times the $D L$ receive $F=$ fail.

- A conversion from $\mathrm{lb} / 10^{12}$ Btu to $\mathrm{kg} / \mathrm{J}$ should be footnoted in the table. 
8. Value was discarded using Dixon's criterion for outliers.

9. Value for a stream concentration was calculated by difference rather than by measurement.

10. Mass balance closure for entire system was outside the range of $80 \%-120 \%$ for major elements (Al, $\mathrm{Ca}, \mathrm{Fe}, \mathrm{Mg}$, and $\mathrm{Ti}$ ) or $70 \%-130 \%$ for trace elements $(\mathrm{Sb}, \mathrm{As}, \mathrm{Ba}, \mathrm{Be}, \mathrm{B}, \mathrm{Cd}, \mathrm{Cl}, \mathrm{Cr}, \mathrm{Co}, \mathrm{Cu}, \mathrm{F}, \mathrm{Pb}, \mathrm{Mn}, \mathrm{Hg}, \mathrm{Mo}$, $\mathrm{Ni}, \mathrm{Se}$, and V).

Number of Valid Measurements Needed and Nondetect Values. At least three valid measurements are needed to evaluate data statistically. A minimum of four measurements is preferred to perform Dixon's criterion for outliers (35), where at least one erroneous data point can be eliminated, leaving at least three data points on which to base statistical calculations or scientific conclusions. For the Phase I sampling effort, however, only three measurements were taken at each sampling location. Data eliminated through the Dixon's criterion for outliers procedure should still be reported in the data tables and flagged accordingly. Also, reporting of nondetect values as one-half the $D L$ is not valid and could lead to future misuse or misunderstanding of the data. For the purpose of reporting concentrations and emission factors, where all values are nondetects, they should be reported as $\mathrm{ND}<\mathrm{DL}=\mathbf{x} \cdot \mathrm{xx}$.

Analysis of Blanks. Analysis of each blank must indicate $N D \leq D L=x \cdot x x$ or the presence of no more than $25 \%$ of the mean value of that analyte detected in the samples. For the purpose of gathering quantitative data for HAP emission characterization, high blanks invalidate sampling results. Therefore, a suggested value of $25 \%$ was chosen as an upper bound for blank data.

Spike Recovery. In Phase I, recoveries of spikes and surrogates were required to meet the standards as defined by the given method. The EERC recommends that, in addition to meeting the method-defined standard, spike recoveries be between $70 \%$ and $125 \%$. While spike recoveries outside the EERC-defined range may be generally accepted for a given method, it is the EERC's opinion that such data do not yield information useful for HAP emission quantitation and regulation. Depending upon the 
portion of the analytical method that fails a spike recovery le.g., the filter or front half catches for Method 29), the effect on the data can be an artificially high or low result. Interpretation of this effect requires more information than is available on the table, so a "pass/fail" designation was used on the table. Where the blank or spike recoveries have failed, the subsequent effect on the data should be explained in the text of the report. Specific explanations for failed $Q A / Q C$ data were not consistently provided in the Phase I site reports.

Detection Limit and Lower Limit of Quantitation. Having data above the DL of the analytical methods used is another criterion for data validation. In addition, the EERC contends that all data below the LLQ, defined as 10 times the DL, do not yield the highest-quality, most defensible data for the purpose of HAP emission quantitation and regulation. Therefore, both categories, "Above $\mathrm{DL}$ " and "Above LLQ" were flagged with either a $P=$ pass or $F=$ fail. Data failing the LLQ but passing the DL are valid but are presented in the context that they are of weaker quality than those that pass both the LLQ and the DL criteria.

\subsubsection{Mercury Speciation and Emission Factors}

The quality of some of the DOE Phase I Hg data is questionable because of its failure to pass one or more of the $\mathrm{QA} / \mathrm{OC}$ criteria or the absence of $\mathrm{QA} / \mathrm{OC}$ data to permit an evaluation of data quality. Blank concentration data were not reported for one of 11 EPA Method 29 data sets and for six of the $20 \mathrm{Hg}$ data sets overall. QA/OC failure because of high blank concentrations was observed for one of 11 EPA Method 29 data sets. Blank concentration data, where reported, for the alternative $\mathrm{Hg}$ sampling methods (hazardous element sampling train [HEST], mercury speciation absorption [MESA] method, or Brooks-Rand sorbent traps) passed QA/QC criteria. Spike recovery (internal or audit spike) failures were observed for eight of the 11 EPA Method 29 data sets. Spike recovery $Q A / Q C$ data were not required for the alternative Hg-sampling methods but, nevertheless, should be addressed in Phase II. Data quality was also reviewed with respect to DL and LLQ for EPA Method 29 data. Detection limits were not reported for two of the 11 data sets. However, the remaining nine data sets passed the DL criterion of which seven passed the LLQ. 
Caution must be exercised in the use of these $\mathrm{Hg}$ results with respect to a large population of coal-fired boilers because of the small size of the data set, the large number of variables represented (fuel types, boiler types, emission control systems, etc.), and the questionable quality of some of the data. The remainder of this section will discuss the $\mathrm{Hg}$ data reported and develop conclusions concerning $\mathrm{Hg}$ emissions from coal-fired boilers and the level of control that can be expected from conventional and advanced emission control technologies.

Emission factors and percent penetration data for total $\mathrm{Hg}$ are presented in Figure 4-7. The data are based on EPA Method 29 sampling for seven of the nine process configurations, with sorbent trap data reported for the Cardinal and Springerville Stations. The $\mathrm{Hg}$ emission factor data are presented on a $\mathrm{lb} / 10^{12} \mathrm{Btu}$ basis, with two-sided error bars denoting the $95 \% \mathrm{Cl}$. Emission factors for total $\mathrm{Hg}$ ranged from 1.9 to $22 \mathrm{lb} / 10^{12}$ Btu based on mean stack concentrations that ranged from roughly 2.6 to $30 \mu \mathrm{g} / \mathrm{Nm}^{3}$. Mean $\mathrm{Hg}$ concentration in coal ranged from 0.04 to $0.28 \mu \mathrm{g} / \mathrm{g}$, and typical mean values of about $0.1 \mu \mathrm{g} / \mathrm{g}$ were reported for six of the nine process configurations. The highest mean $\mathrm{Hg}$ emission factors were reported for the Niles Station (14 $\left.\mathrm{lb} / 10^{12} \mathrm{Btu}\right)$ and SNOX process $\left(22 \mathrm{lb} / 10^{12} \mathrm{Btu}\right.$, located at the Niles Station), which also reported the highest mean $\mathrm{Hg}$ concentrations in the coal, 0.22 and $0.28 \mu \mathrm{g} / \mathrm{g}$, respectively. The lowest mean coal $\mathrm{Hg}$ concentration $(0.04 \mu \mathrm{g} / \mathrm{g})$ was reported for the Springerville Station. However, the mean emission factor for the Springerville Station (4 $\left.\mathrm{lb} / 10^{12} \mathrm{Btu}\right)$ was on the high end of the range $(1.9$ to $\left.4 \mathrm{lb} / 10^{12} \mathrm{Btu}\right)$ for five other sites which had coal $\mathrm{Hg}$ concentrations of roughly $0.1 \mu \mathrm{g} / \mathrm{g}$. The mean $\mathrm{Hg}$ emission factors for the Cardinal $\left(8.5 \mathrm{lb} / 10^{12} \mathrm{Btu}\right)$ and Coal Creek Stations $\left(9.5 \mathrm{lb} / 10^{12} \mathrm{Btu}\right)$ were high relative to four other sites reporting $\mathrm{Hg}$ concentrations in the coal of $0.1 \mu \mathrm{g} / \mathrm{g}$. However, there is overlap in the $95 \%$ confidence intervals reported for these sites, with the Cardinal and Coal Creek Stations having the largest confidence intervals of the group. Based on fuel feed rates ( 37 to 560 tons/hr) and analyses (6230 to $12,200 \mathrm{Btu} / \mathrm{lb}$ ) reported for the nine process configurations and an overall average capacity factor of 0.7 , the estimated annual emission rates for total $\mathrm{Hg}$ ranged from 0.004 to 0.2 tons/yr, with an average emission rate for the nine process configurations of 0.07 tons/yr. 


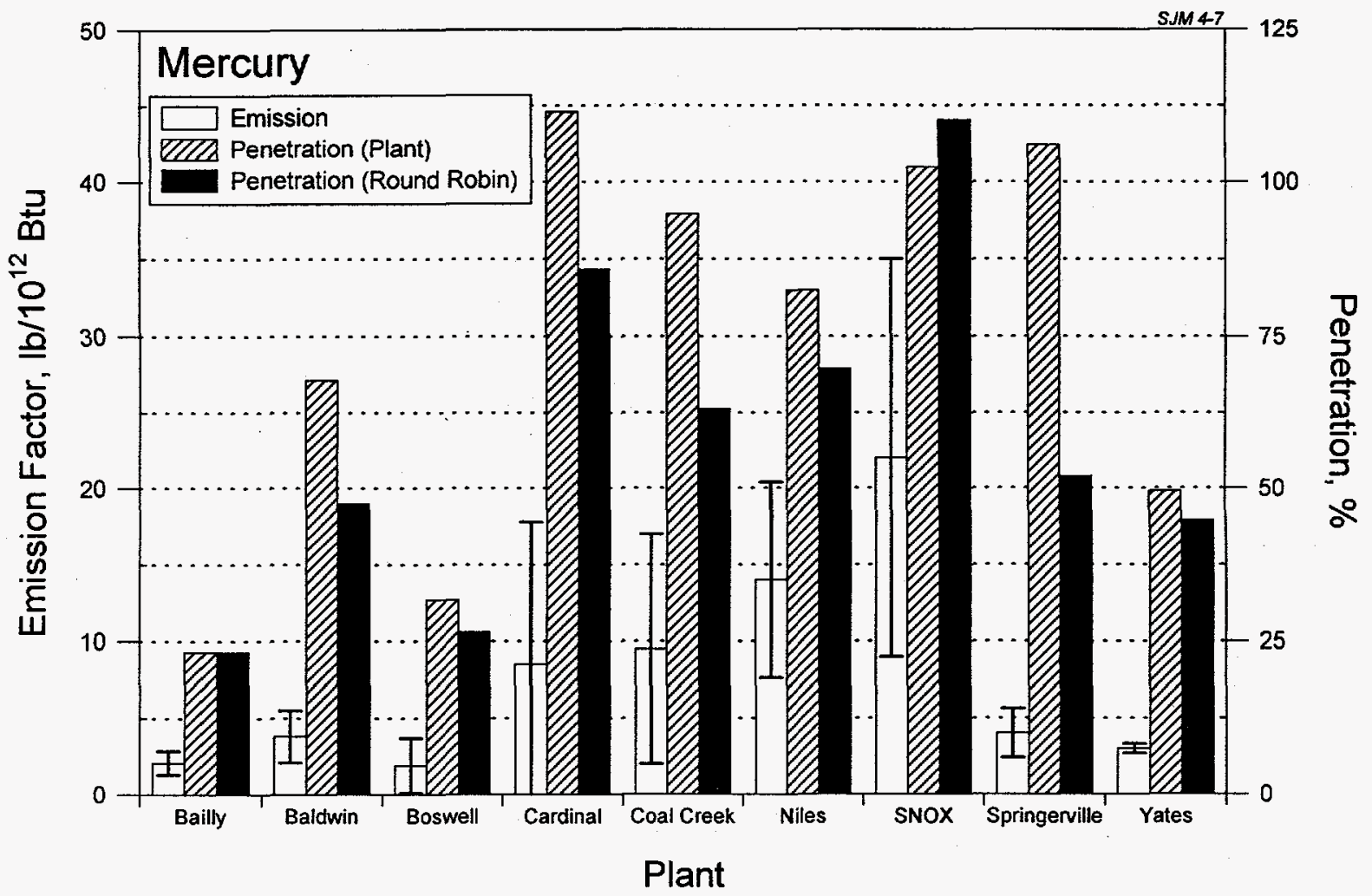

Figure 4-7. Emissión Factor and Penetration Data for Mercury.

Penetration data also demonstrate a significant degree of variability and range in control for total $\mathrm{Hg}$. Calculated percent penetration values for total $\mathrm{Hg}$ ranged from about $25 \%$ to $112 \%$. Two sets of penetration data are presented, one set based on plant coal data and the second on round-robin coal data. Three of the nine process configurations had percent penetration values $>100 \%$ based on plant coal data. Three sites had $\mathrm{Hg}$ penetration values of $65 \%$ to $95 \%$ (35\% to $5 \%$ control), with penetration values for the remaining three sites of $<50 \%$ ( $>50 \%$ control). Two of the sites with $\mathrm{Hg}$ penetration values of $>100 \%$ represented a combination of particulate and acid gas control technologies, with the third site employing only an ESP for emission control. The three sites with $\mathrm{Hg}$ penetration values of $<50 \%$ ( $>50 \%$ control) employed a FF as the only emission control technology in one case and ESP-FGD combinations in two cases. The three remaining sites $165 \%$ to $95 \%$ penetration) employed ESPs at two sites and an ESP-wet FGD with $40 \%$ bypass at the third. 
Penetration data based on round-robin coal data only resulted in one site having a penetration value exceeding $100 \%$ : the SNOX process. Since the emission factor for the SNOX process had the largest $\mathrm{Cl}$, data quality and variability appear to play a significant role in the result. Four sites had $\mathrm{Hg}$ penetration values of $50 \%$ to $85 \%$ (50\% to $15 \%$ control) with penetration values for the remaining four sites of $<50 \%$ (>50\% control). Two of the four sites having $\mathrm{Hg}$ penetration values of $50 \%$ to $70 \%$ (50\% to $30 \%$ control) employed an ESP as the only emission control technology with the other two sites representing an ESP-FGD and a spray dryer-FF combination, respectively. The four sites with $\mathrm{Hg}$ penetration values of $<50 \%$ ( $>50 \%$ control) employed ESP-FGD combinations in two cases, a FF in one and an ESP in another.

From the DOE Phase I data, it is not possible to clearly delineate the potential to control $\mathrm{Hg}$ emissions from coal-fired utility boilers using existing emission control technologies. Because of the overlap of the Cls, no statistically valid differences can be identified in the emission factor data beyond gross coal $\mathrm{Hg}$ concentration effects. Also, the $\mathrm{Hg}$ penetration data appear to be influenced as much by variations in the coal $\mathrm{Hg}$ concentrations as by the emission control technology employed. It is also important to remember that the control of volatile or vapor-phase HAPs such as $\mathrm{Hg}$ is not likely to exceed control levels for vapor-phase priority pollutants such as $\mathrm{SO}_{2}$.

Evaluating emission control technology performance based on total $\mathrm{Hg}$ concentration alone is not appropriate, since $\mathrm{Hg}$ speciation may affect the degree of control observed. This effect currently cannot be quantified adequately, since methods to speciate $\mathrm{Hg}$ are still unproven. Future $\mathrm{Hg}$-sampling efforts must emphasize accurate $\mathrm{Hg}$ speciation in order to evaluate the performance of emission control technologies properly.

\subsubsection{Other Volatile Trace Elements}

For the purposes of this report, other volatile trace elements include B and Se. Boron data were reported for only seven of the nine process configurations. The quality of the $B$ data is questionable for several sites because of the limited amount of QA/OC criteria data presented and blank and/or spike recovery failures were noted for 
two of nine data sets reported. Blank concentration data were not reported for two of nine EPA Method 29 data sets. For the Niles Station and the SNOX process, B data were not reported because of sample preparation interferences. Internal spike recovery failures were observed for two of the nine data sets, and no spike recovery QA/QC data were reported for three additional data sets. Data quality was also reviewed with respect to DL and LLQ. Detection limits were reported for only one $B$ data set, which passed the DL and LLQ criteria. However, the high B concentrations reported suggest that all of the data exceed DL as well as LLO criteria.

Emission factors and penetration data for total $B$ are presented in Figure 4-8 for six DOE Phase I field sites. The B emission factors data are presented on a $\mathrm{lb} / 10^{12}$ Btu basis with two-sided error bars denoting the $95 \% \mathrm{Cl}$. Emission factors for total $B$ ranged from 19 to $7700 \mathrm{lb} / 10^{12}$ Btu based on mean stack concentrations that ranged from roughly 26 to $10,454 \mu \mathrm{g} / \mathrm{Nm}^{3}$. Mean $\mathrm{B}$ concentration values in coal ranged from about 56 to $302 \mu \mathrm{g} / \mathrm{g}$ for the plant coal data and 70 to $230 \mu \mathrm{g} / \mathrm{g}$ for the round-robin coal data. The highest mean $B$ emission factor $\left(7700 \mathrm{lb} / 10^{12} \mathrm{Btu}\right)$ was reported for the Baldwin Station (ESP, 95.6\%-98.1\% particulate control), which had a high $(185 \mu \mathrm{g} / \mathrm{g})$ plant coal B concentration and the highest mean coal B concentration $(230 \mu \mathrm{g} / \mathrm{g})$ reported for the round-robin coal data. The lowest mean coal B concentration was reported for the SNOX process, roughly $56 \mu \mathrm{g} / \mathrm{g}$ based on plant coal data. The round-robin coal data indicated a mean $B$ concentration for the Niles Station and SNOX process of roughly $71 \mu \mathrm{g} / \mathrm{g}$, with three other sites having mean B concentrations of 72 to $83 \mu \mathrm{g} / \mathrm{g}$. However, emission factors for the Niles Station and SNOX process were not reported. The lowest mean B emission factor $\left(19 \mathrm{lb} / 10^{12} \mathrm{Btu}\right)$ was reported for the Coal Creek Station (ESP-wet FGD with $40 \%$ bypass, $99.8 \%$ particulate controll with mean coal B concentrations of 174 and $130 \mu \mathrm{g} / \mathrm{g}$ based on plant and round-robin coal data, respectively.

Penetration data also demonstrate a significant degree of variability and range in control for total $B$. Calculated percent penetration values for total $B$ ranged from $<1 \%$ to roughly $50 \%$ ( $>99 \%$ to $50 \%$ control) based on plant coal data. Two of the six sites had percent penetration values of $>30 \%(<70 \%$ control), with penetration values for the remaining four sites of $<10 \% \cdot(>90 \%$ control). The two sites with $B$ 


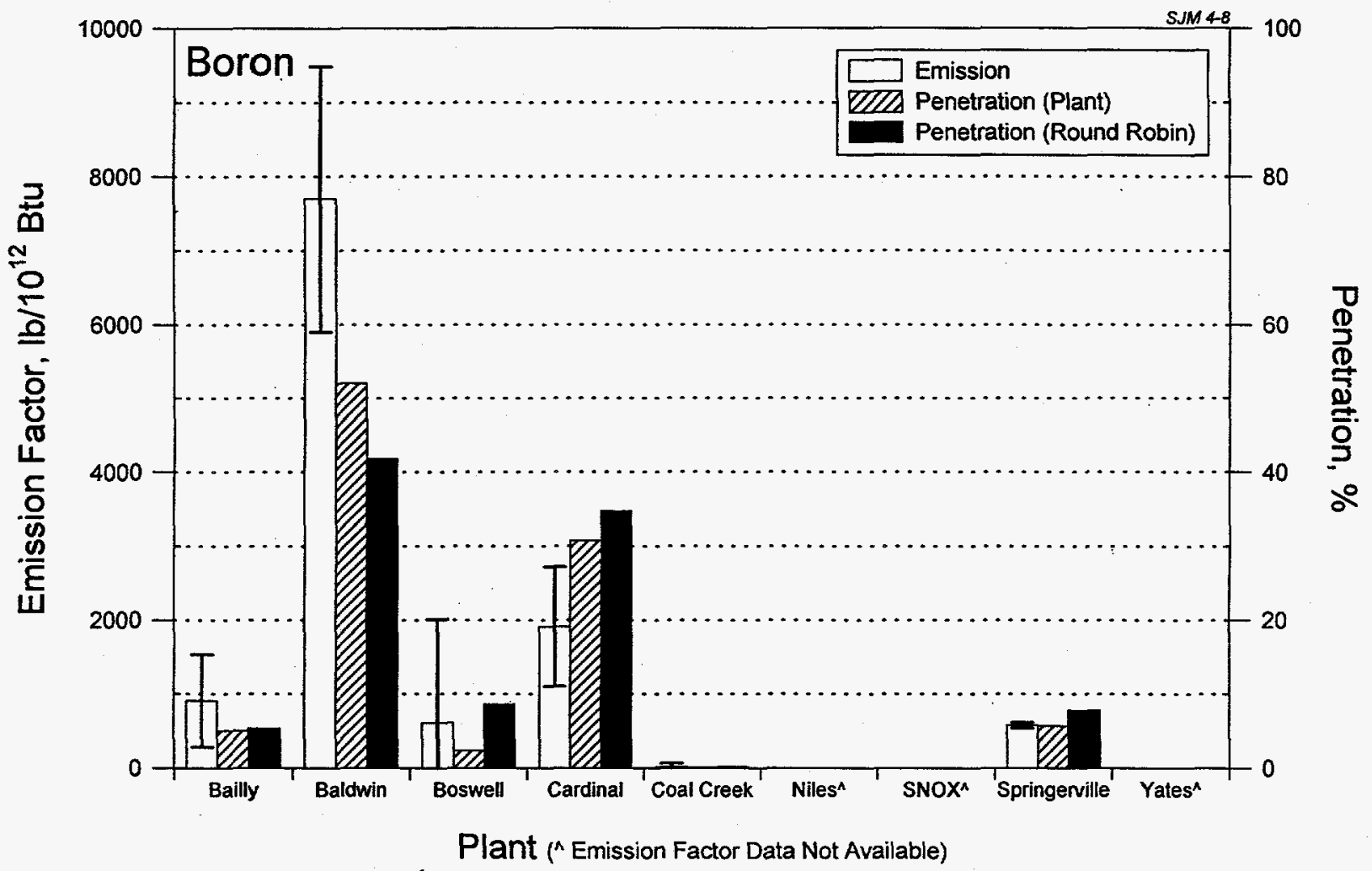

Figure 4-8. Emission Factor and Penetration Data for Boron.

penetration values of $>30 \%$ employed only an ESP for emission control technologies. The four sites with B penetration values of $<10 \%$ ( $>90 \%$ control) employed either a FF, SDA-FF, or an ESP-FGD combination. Calculating penetration data based on round-robin coal data had a relatively small effect on the results. For the Baldwin Station, percent penetration decreased from roughly $50 \%$ to $40 \%$. These data indicate that $B$ emissions were effectively controlled at sites where overall particulate control was $>99.5 \%$. In the DOE Phase I field sampling effort, effective B control was observed only for sites employing either a FF or a combination of particulate and acid gas control technology.

Fourteen sets of Se data were reported for the nine process configurations. These included 11 data sets based on EPA Method 29 sampling and three data sets based on the HEST method. This discussion will focus on the EPA Method 29 data. Blank and/or spike recovery failures were noted for ten of the 11 data sets reported. 
Blank concentration data were not reported for one data set. However, the reported stack concentrations were $\angle D L$. QA/OC failure because of high blank concentrations were reported for two of the 11 data sets. Internal spike recovery failures were observed for six of the 11 data sets, and only one site passed the audit spike recovery criteria. Data quality was also reviewed with respect to DL and LLQ for EPA Method 29 data. Detection limits were reported for seven Se data sets that passed the DL criterion of which six passed the LLQ. Figure 4-9 illustrates Se emission factors and penetration data.

Emission factors for total Se ranged from ND $<0.038$ to $193 \mathrm{lb} / 10^{12}$ Btu based on mean stack concentrations that ranged from ND $<0.032$ to $261 \mu \mathrm{g} / \mathrm{Nm}^{3}$. Mean Se concentration values in coal ranged from ND $<0.64$ to $3.7 \mu \mathrm{g} / \mathrm{g}$ and from 0.80 to $3.2 \mu \mathrm{g} / \mathrm{g}$ for plant and round-robin coal data, respectively. The highest mean Se emission factor $\left(193 \mathrm{lb} / 10^{12} \mathrm{Btu}\right)$ and the largest $\mathrm{Cl}$ were reported for the Bailly Station (ESP-wet FGD), which had a relatively high mean coal Se concentration $(1.5 \mu \mathrm{g} / \mathrm{g})$ based on plant coal data and the highest value $(3.2 \mu \mathrm{g} / \mathrm{g})$ based on roundrobin coal data. The lowest mean coal Se concentration, based on plant coal data, was reported for the Niles Station (ND $<0.64 \mu \mathrm{g} / \mathrm{g}$ ). However, the round-robin coal data indicated a relatively high mean Se concentration $(2.6 \mu \mathrm{g} / \mathrm{g})$ for the Niles Station. Also, the mean emission factor $\left(62 \mathrm{lb} / 10^{12} \mathrm{Btu}\right)$ for the Niles Station (ESP) was not the lowest emission factor reported. The lowest mean Se emission factor (ND $<0.038 \mathrm{lb} / 10^{12} \mathrm{Btu}$ ) was reported for the Springerville Station (SDA-FF) with a mean coal Se concentration of $0.9 \mu \mathrm{g} / \mathrm{g}$ based on plant coal data and $1.2 \mu \mathrm{g} / \mathrm{g}$ based on round-robin coal data. Based on fuel feed rates ( 37 to 560 tons/hr) and analyses $(6230$ to $12,200 \mathrm{Btu} / \mathrm{lb})$ reported for the nine process configurations and an overall average capacity factor of 0.7 , the estimated annual emission rates for Se ranged from $<0.001$ to 3.02 tons/yr, with an average emission rate for the nine process configurations of 0.84 tons/yr.

Penetration data also demonstrate a significant degree of variability and range of control for total Se. Calculated percent penetration values for total Se ranged from $<1 \%$ to $>160 \%$ based on plant coal data. Two of the nine process configurations had percent penetration values of roughly $125 \%$ and $165 \%$, with flue gas and coal 


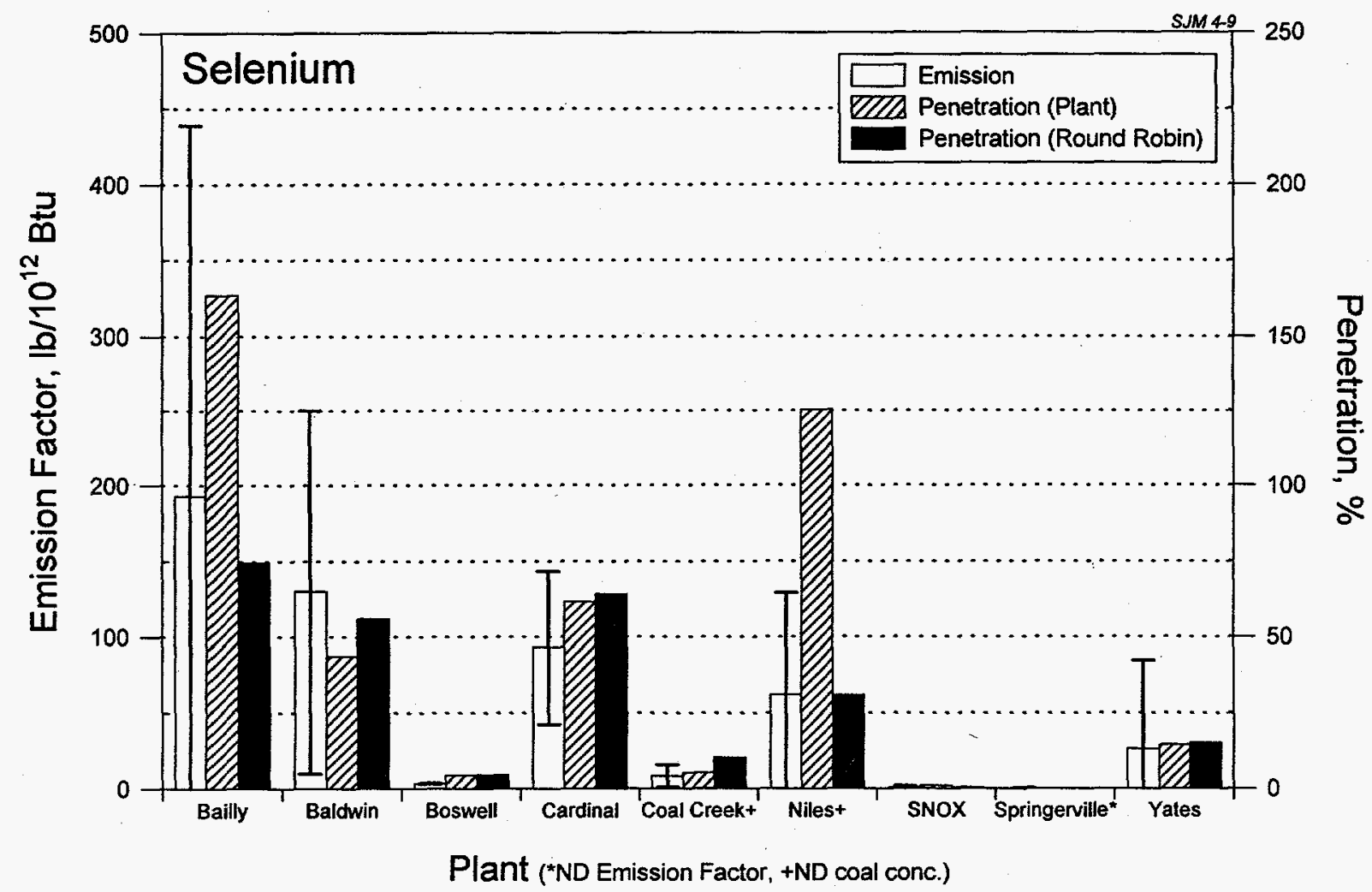

Figure 4-9. Emission Factor and Penetration Data for Selenium.

data variability and quality playing a significant factor in the results. The effect of coal data is apparent when penetration is calculated based on round-robin coal data. For the Bailly Station, percent penetration decreased from $>160 \%$ to $75 \%$. A similar effect was noted for the Niles Station when percent penetration decreased from roughly $125 \%$ to $30 \%$. For the remaining seven sites, percent penetration values are comparable for both the plant and round-robin coal data. One site had a Se penetration value of nearly $66 \%$ (34\% control), with a second site at nearly $50 \%$ (50\% control). Three of the four sites with high Se penetration values $(>25 \%)$ employed an ESP as the only emission control technology on-site. In the case of the fourth site (Bailly Station $75 \%$ to $>160 \%$ Se penetration), poor data quality, indicated by failed spike recoveries, and a variable Se concentration $(0.5$ to $4.4 \mu \mathrm{g} / \mathrm{g})$ in the fuel were contributing factors. Therefore, Bailly Station data cannot be used as an indication of typical ESP-FGD Se control performance. For the Niles Station (30\% to $125 \%$ Se penetration), a very low and variable Se concentration (ND $<0.64 \mu \mathrm{g} / \mathrm{g}$ ) 
in the fuel appears to be the contributing factor. The two sites with Se penetration values in the range of $40 \%$ to $65 \%$ employed only an ESP for emission control. Penetration values for the remaining five sites ranged from $<1 \%$ to $15 \%$ (>99\% to $85 \%$ control). These five sites with Se penetration values of $<15 \%$ ( $>85 \%$ control) employed either a FF, an SDA-FF, or an ESP-FGD combination. Selenium control $>90 \%$ was observed only for sites employing either a FF or a combination of particulate and acid gas control technology. For the SNOX process, $>99 \%$ of the Se was in the sulfuric acid (usable by-product).

Based on the inconsistency of the Se data, it is not possible to clearly delineate the potential to control Se emissions from coal-fired utility boilers using existing emission control technologies. Also, the relative value of the B and Se data for developing conclusions for a large population of coal-fired boilers is limited because of the small size of the data set, the large number of variables represented (fuel types, boiler types, emissions control systems), and the variability of some of the data.

\subsubsection{Nonvolatile Trace Elements}

Figures 4-10 through 4-22 illustrate trace element emission factors and calculated percent penetration for the remaining 13 trace elements $(\mathrm{Sb}, \mathrm{As}, \mathrm{Ba}, \mathrm{Be}, \mathrm{Cd}$, $\mathrm{Cr}, \mathrm{Co}, \mathrm{Cu}, \mathrm{Pb}, \mathrm{Mn}, \mathrm{Mo}, \mathrm{Ni}$, and $\mathrm{V}$ ) at each of the nine process configurations based on EPA Method 29 sampling. Although the emission factor data for the nonvolatile trace elements showed significant variability between individual elements at each site as well as for individual elements between sites, substantially less variability was apparent in the penetration data for the nonvolatile trace elements than observed for $\mathrm{Hg}, \mathrm{B}$, and Se. For individual trace elements, the emission factor range was as low as ND $<0.04$ to $1.4 \mathrm{lb} / 10^{12}$ Btu for Be to as high as 0.17 to $162 \mathrm{lb} / 10^{12}$ Btu for Ba. Trace elements having mean emission factors of $<5 \mathrm{lb} / 10^{12}$ Btu included $\mathrm{Sb}, \mathrm{Be}$, and $\mathrm{Cd}$. With the exception of one or two field sites, the mean emission factors for As, $\mathrm{Co}, \mathrm{Cu}, \mathrm{Pb}, \mathrm{Mo}$, and $\mathrm{V}$ were also $<5 \mathrm{lb} / 10^{12} \mathrm{Btu}$. The emission factors for Ba were $<6 \mathrm{lb} / 10^{12} \mathrm{Btu}$ for six of the nine process configurations. Two sites had Ba emission factors of 82 and $162 \mathrm{lb} / 10^{12} \mathrm{Btu}$, respectively, with the remaining site reporting a $\mathrm{Ba}$ emission factor of $13 \mathrm{lb} / 10^{12} \mathrm{Btu}$. Emission factors for $\mathrm{Cr}$ were $\leq 10 \mathrm{lb} / 10^{12} \mathrm{Btu}$ for 


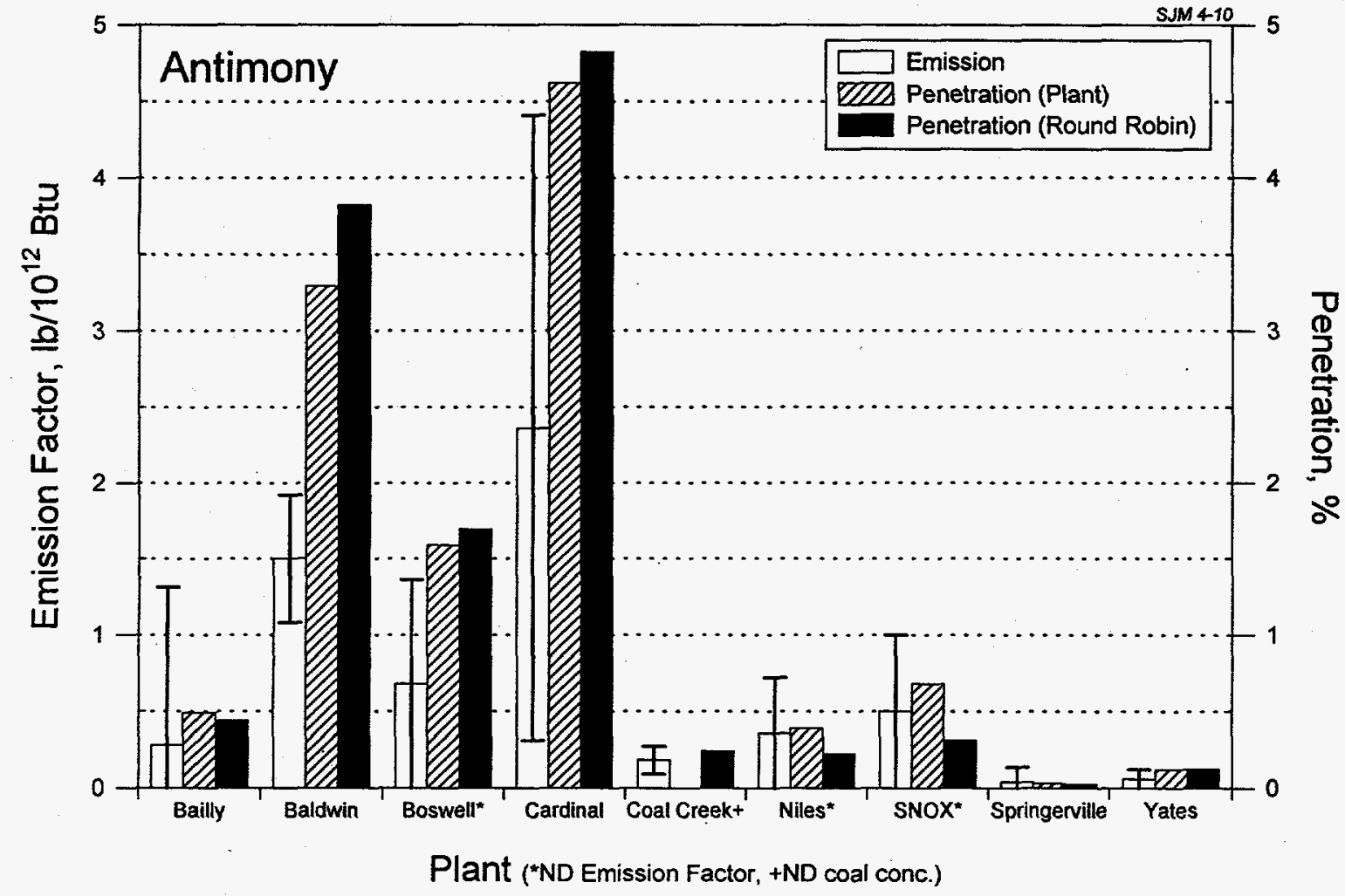

Figure 4-10. Emission Factor and Penetration Data for Antimony.

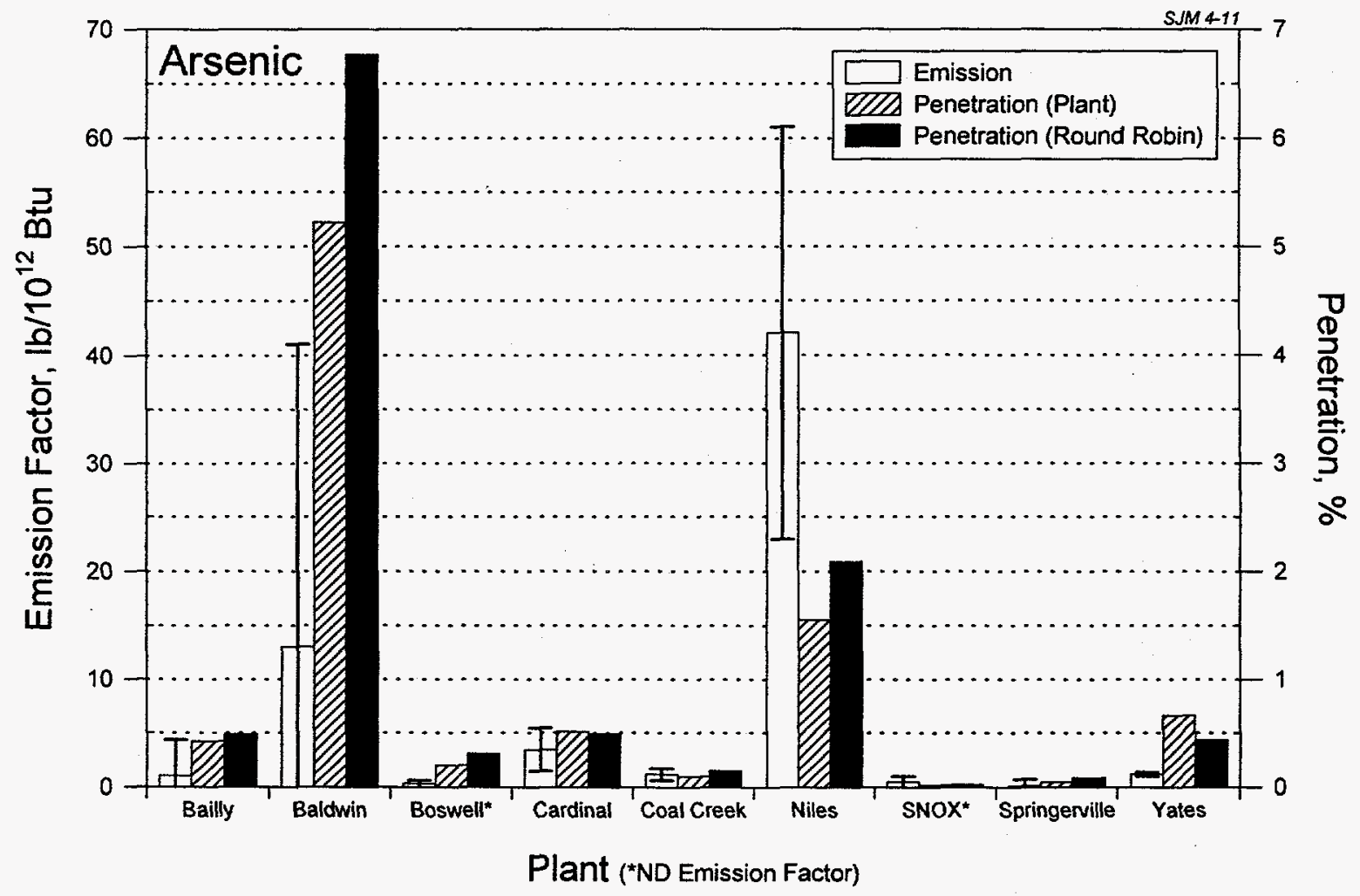

Figure 4-11. Emission Factor and Penetration Data for Arsenic. 


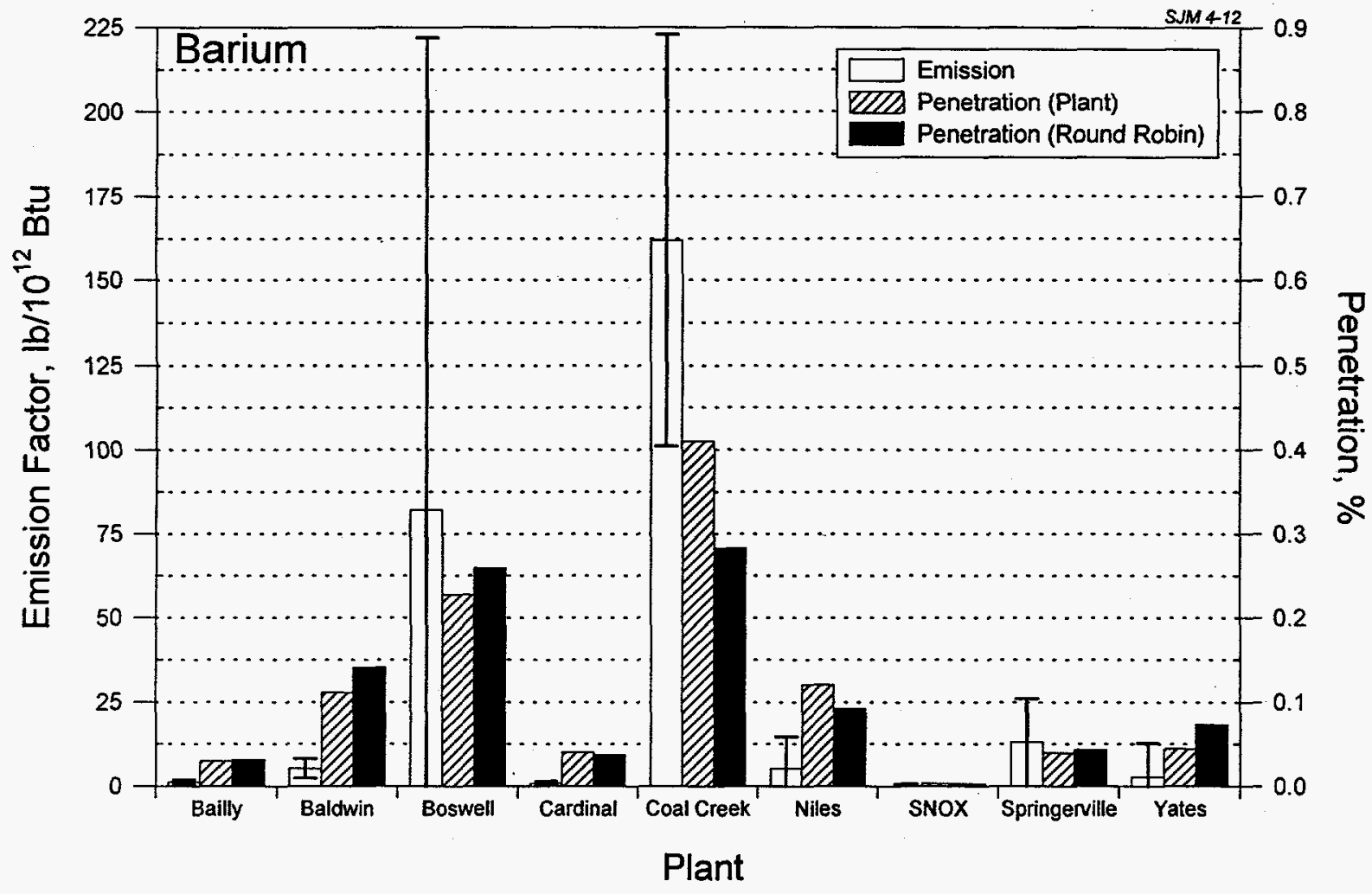

Figure 4-12. Emission Factor and Penetration Data for Barium.

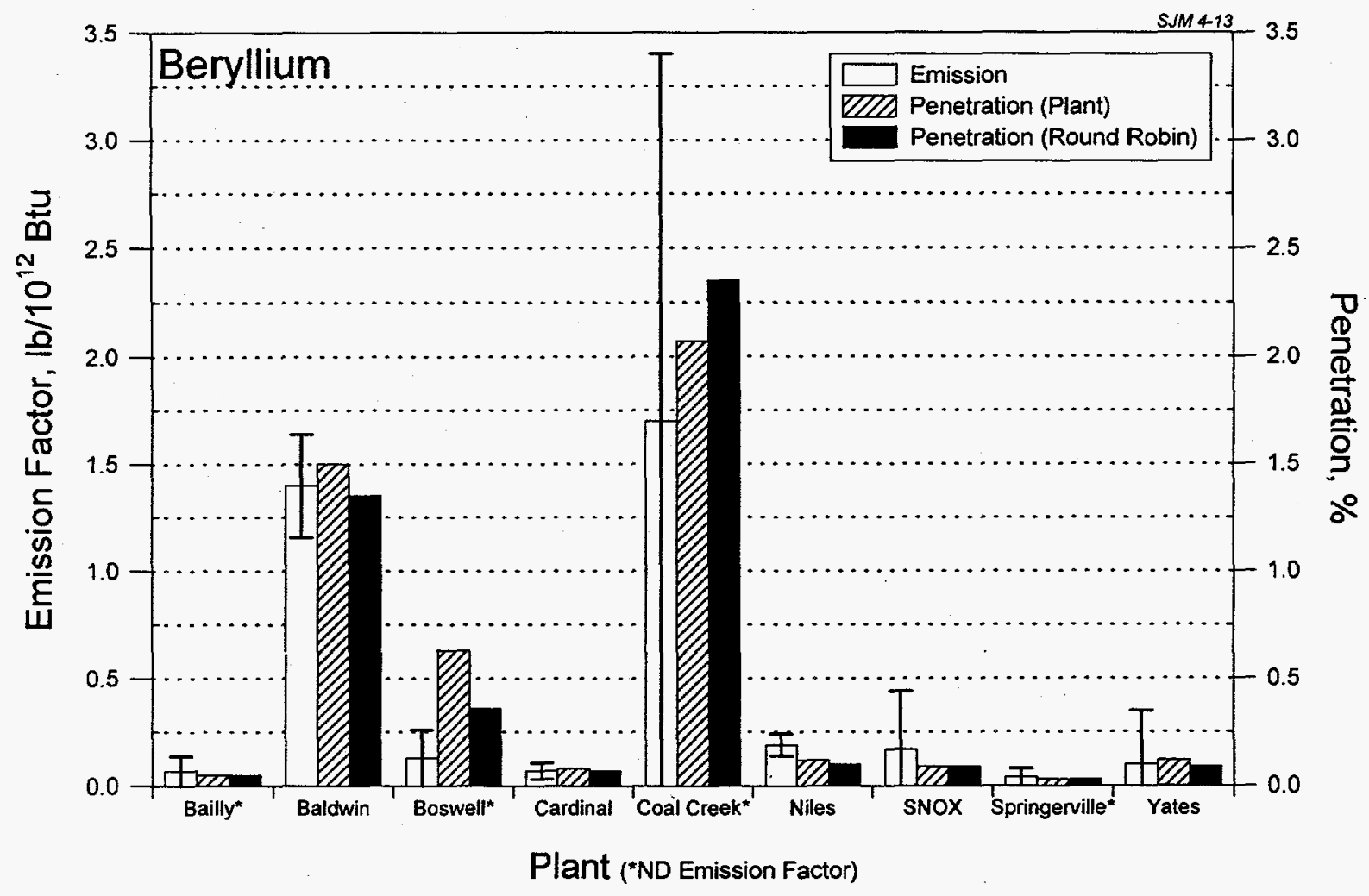

Figure 4-13. Emission Factor and Penetration Data for Beryllium. 


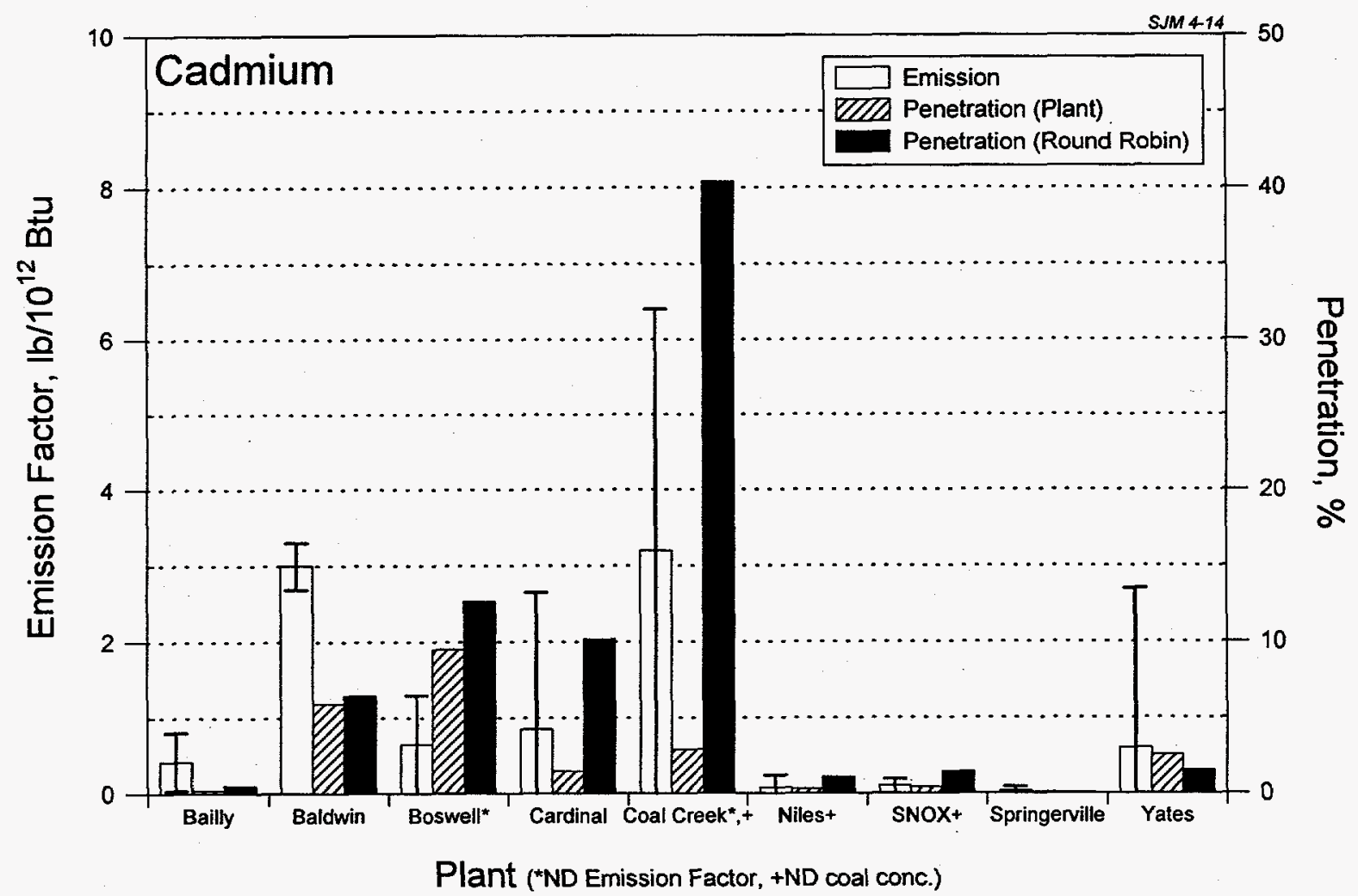

Figure 4-14. Emission Factor and Penetration Data for Cadmium.

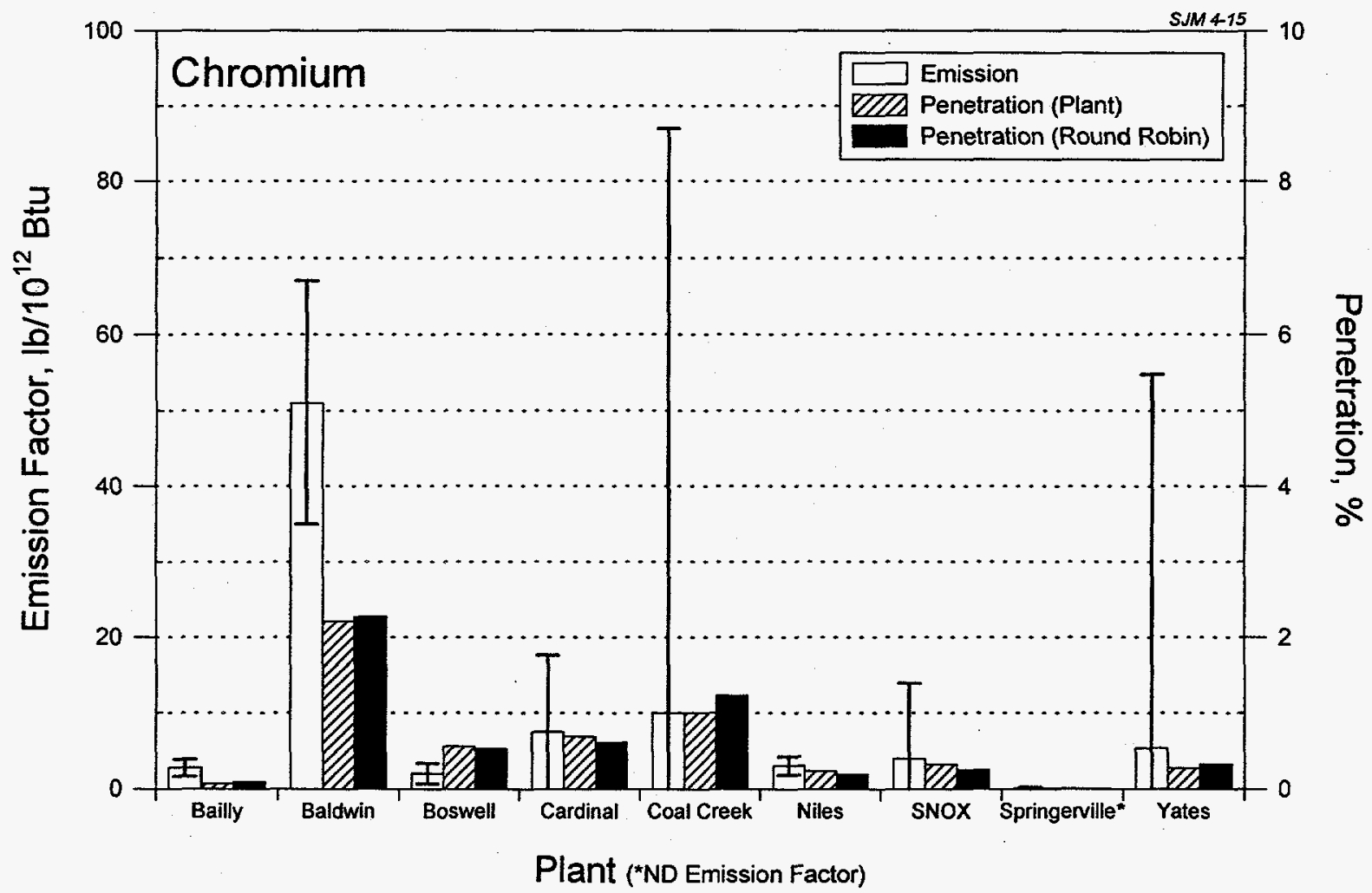

Figure 4-15. Emission Factor and Penetration Data for Chromium. 


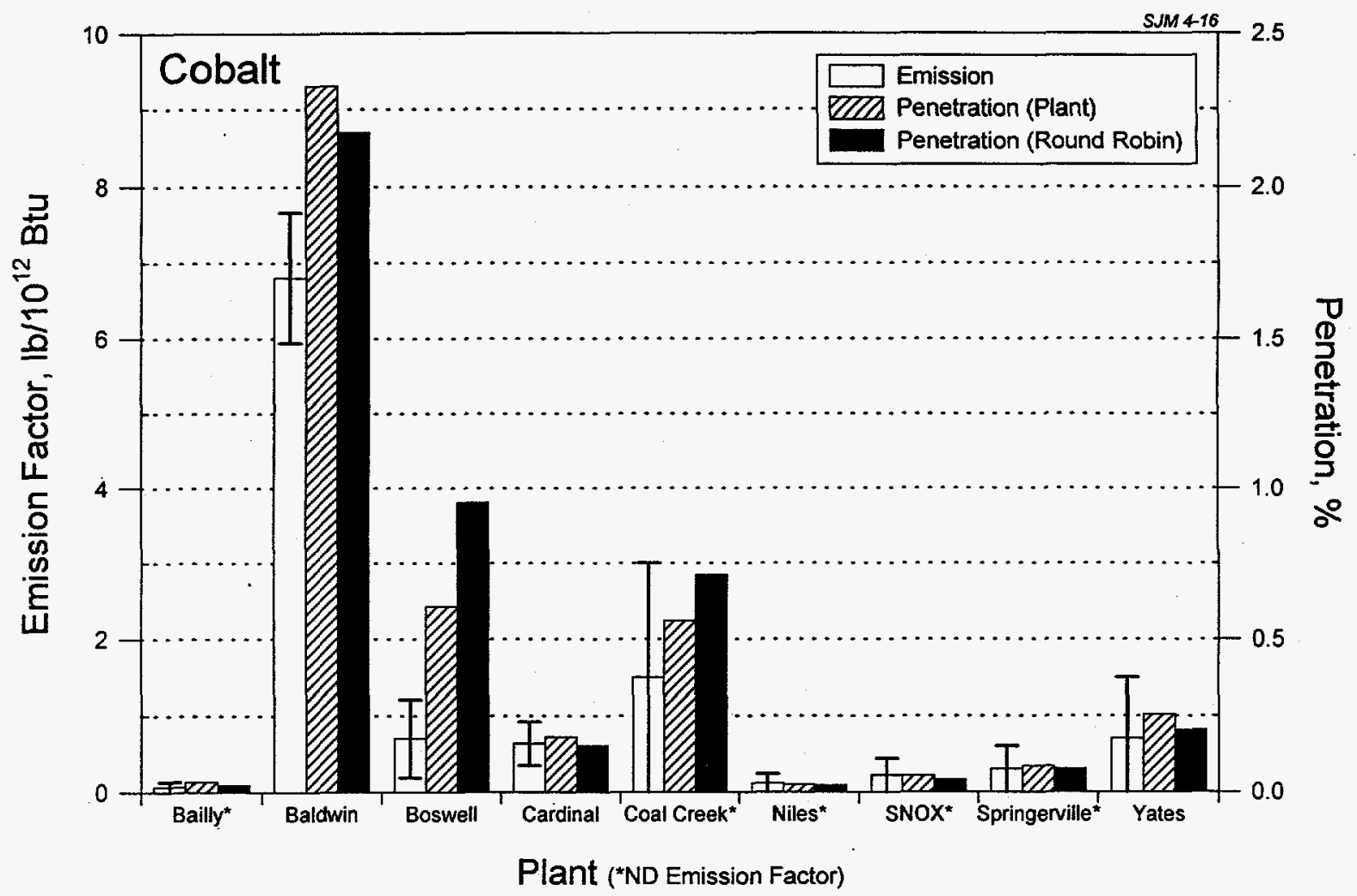

Figure 4-16. Emission Factor and Penetration Data for Cobalt.

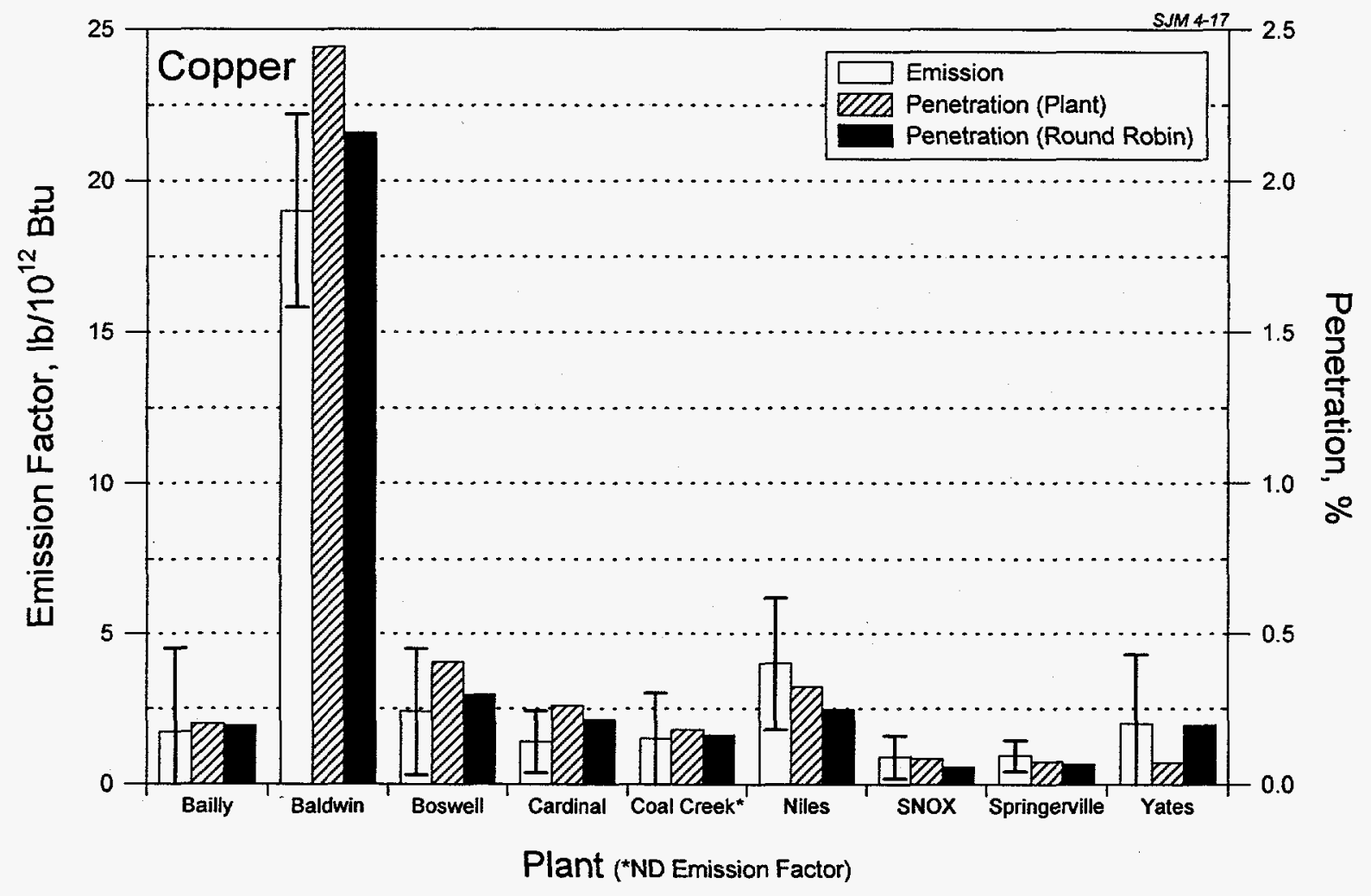

Figure 4-17. Emission Factor and Penetration Data for Copper. 


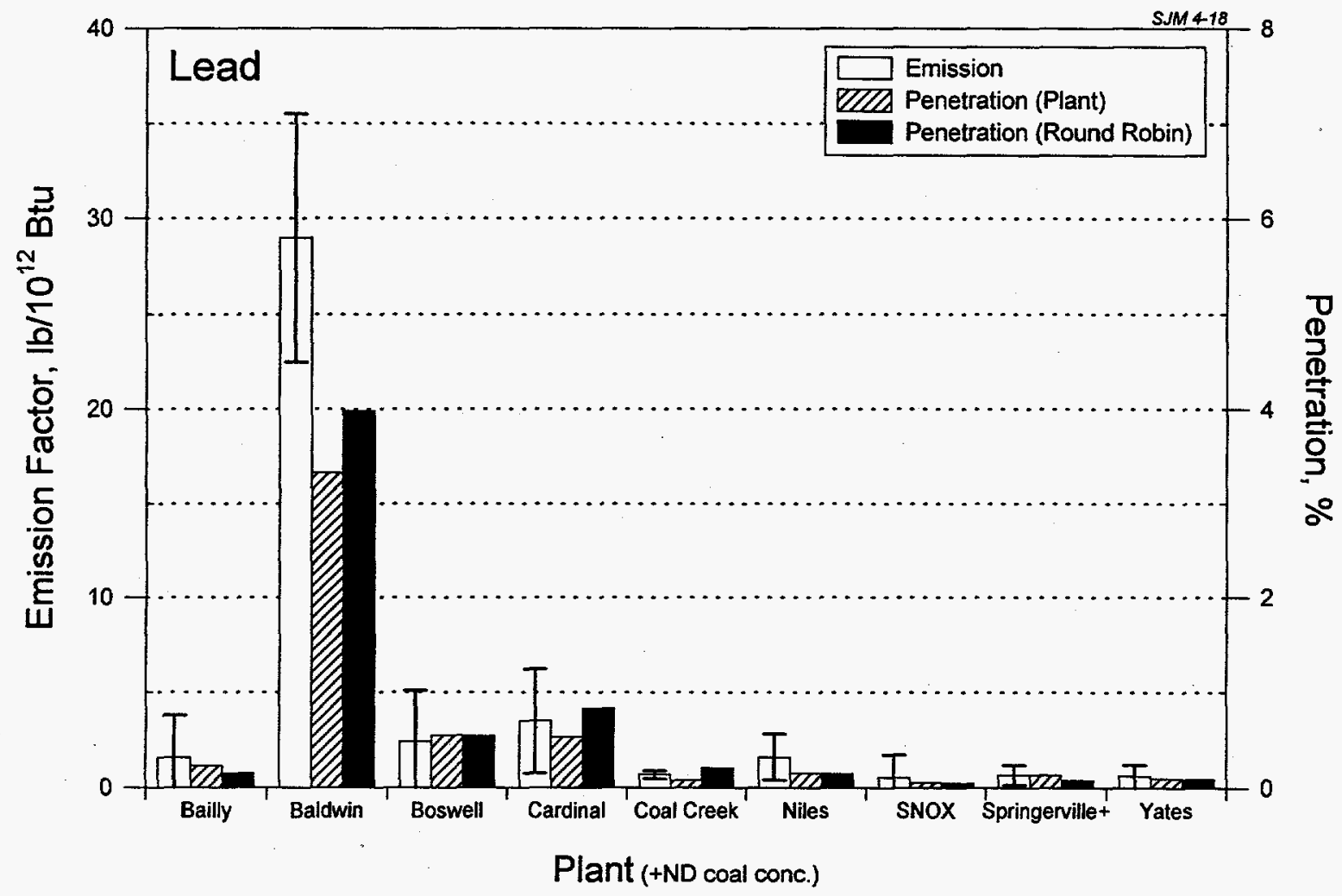

Figure 4-18. Emission Factor and Penetration Data for Lead.

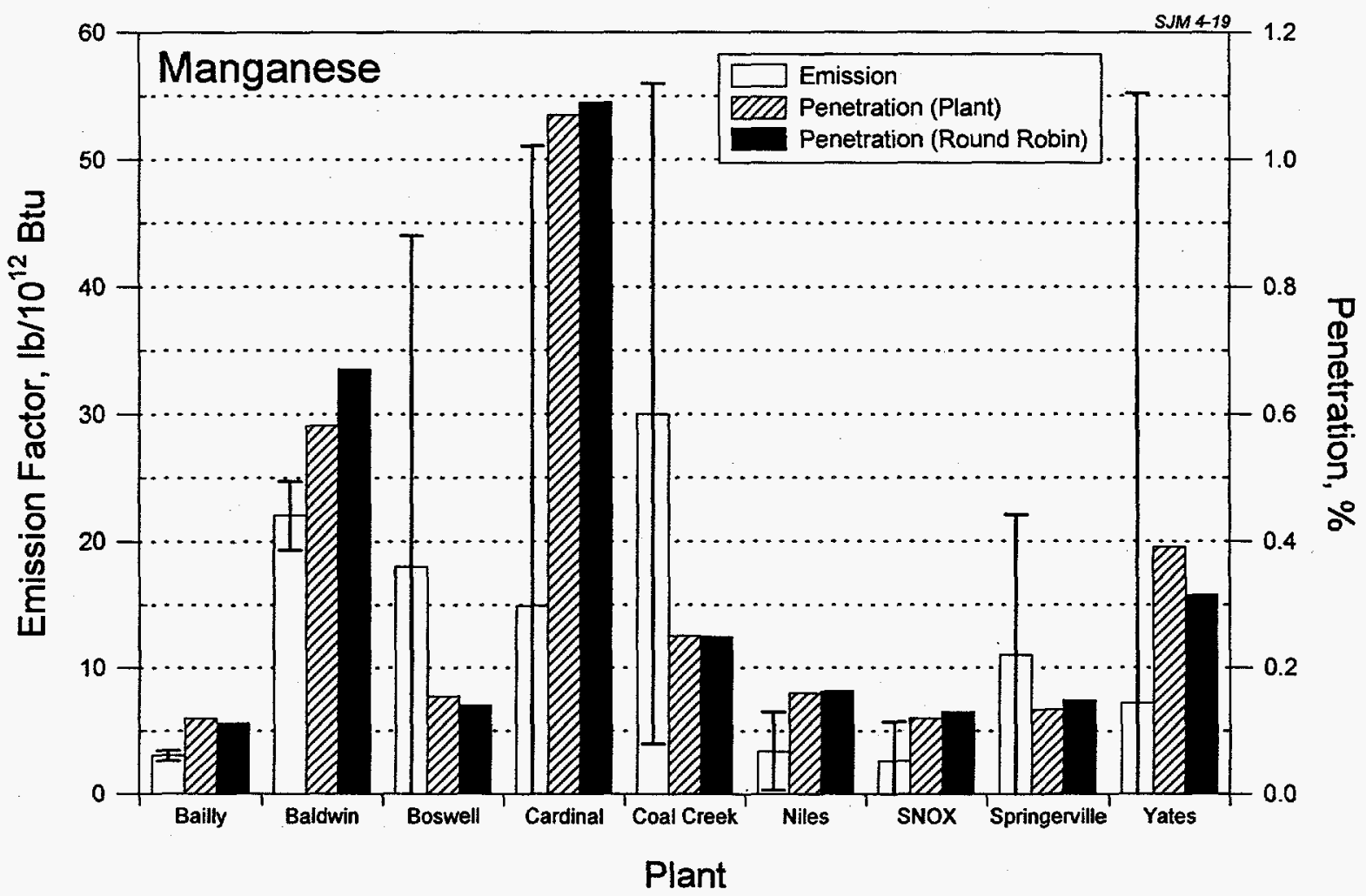

Figure 4-19. Emission Factor and Penetration Data for Manganese. 


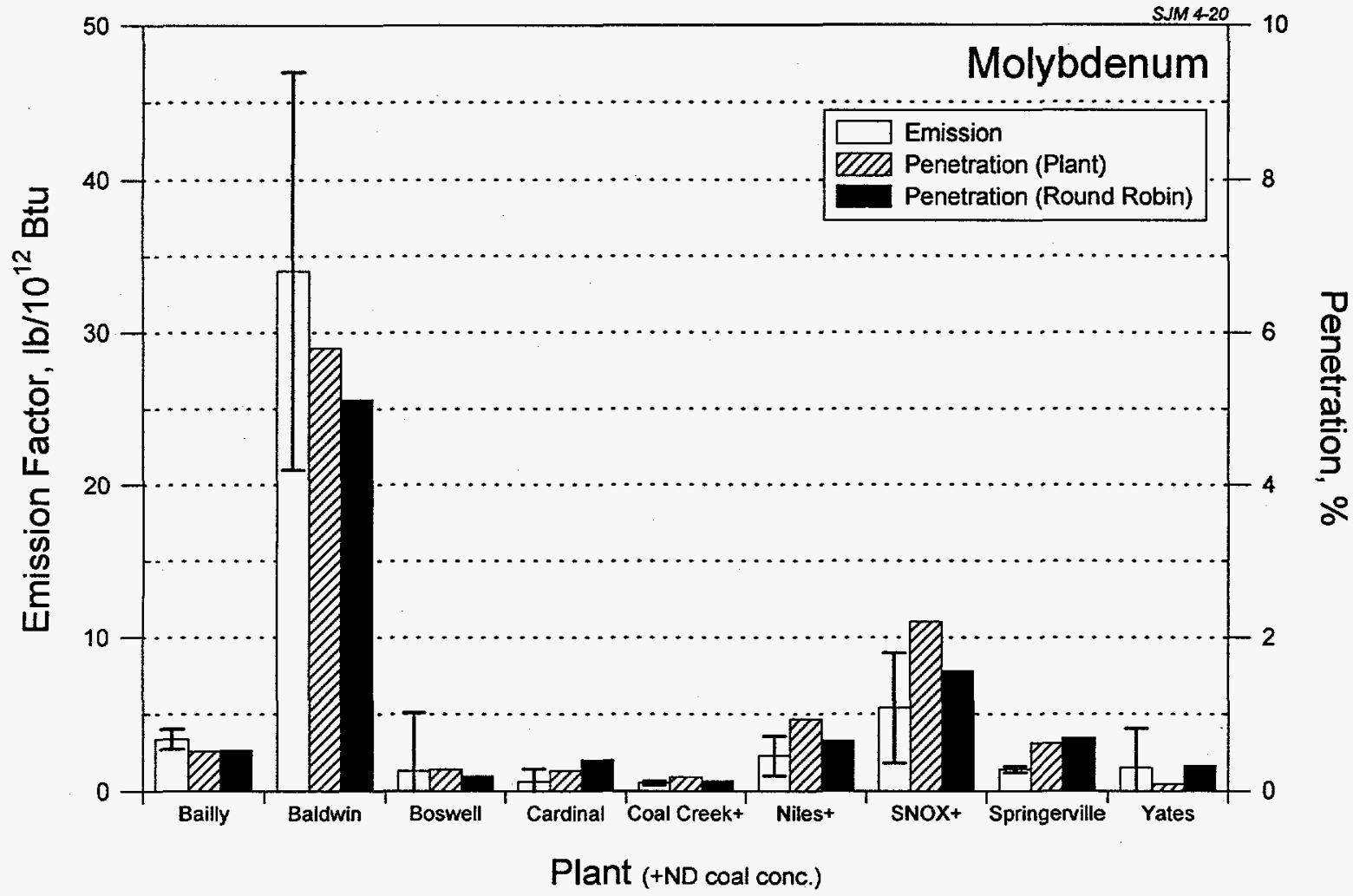

Figure 4-20. Emission Factor and Penetration Data for Molybdenum.

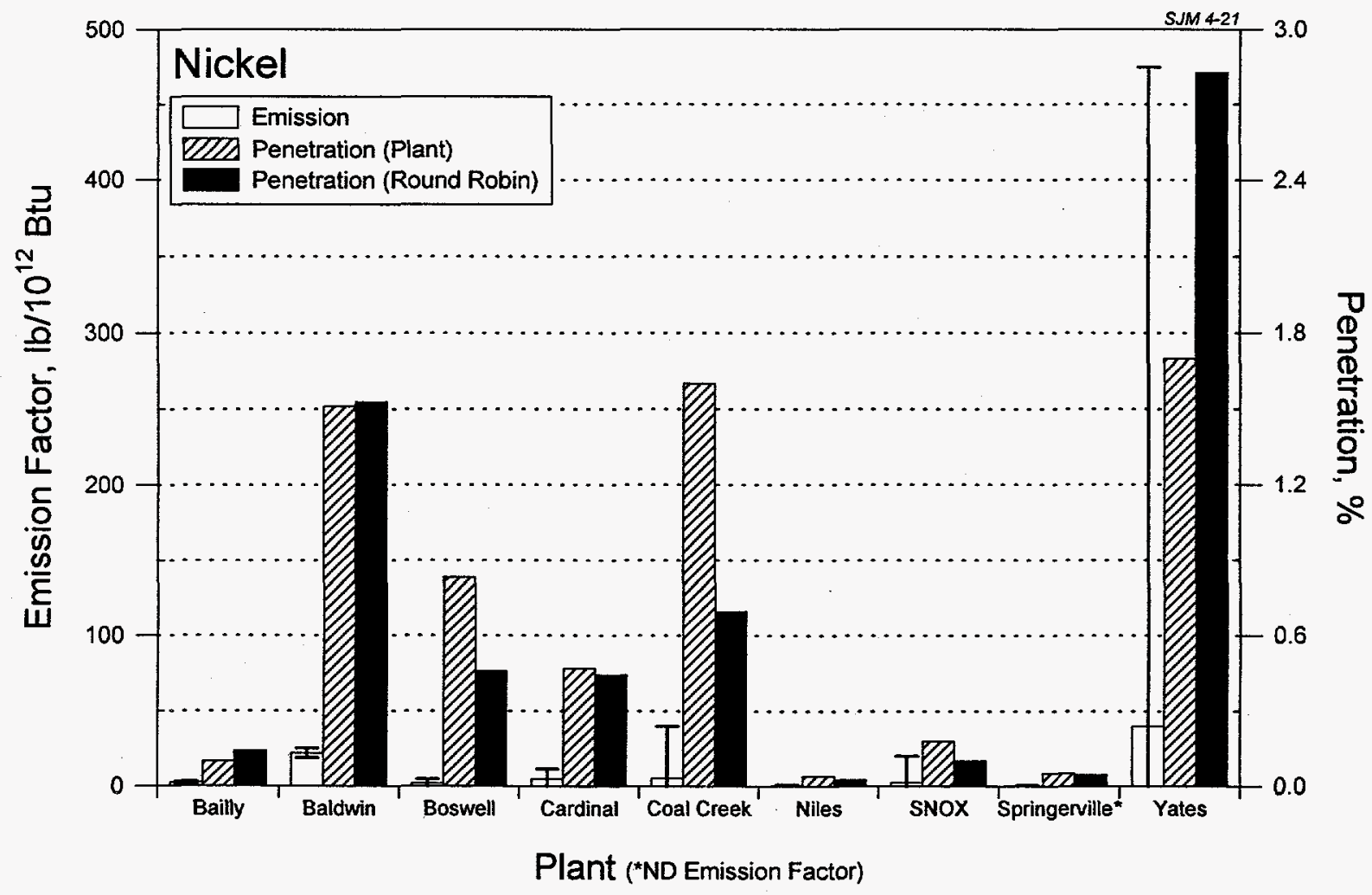

Figure 4-21. Emission Factor and Penetration Data for Nickel. 


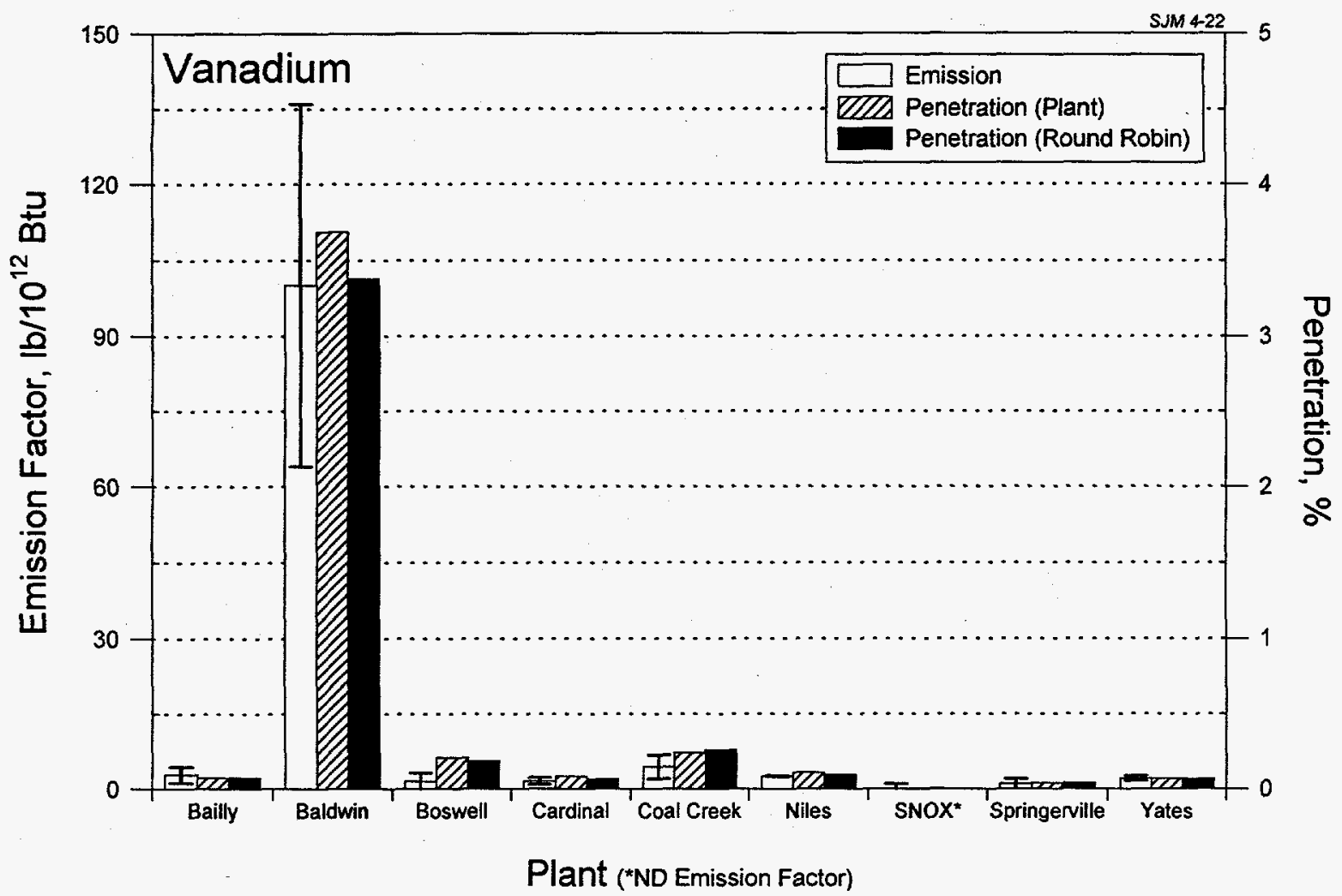

Figure 4-22. Emission Factor and Penetration Data for Vanadium.

eight of nine process configurations, with the ninth having an emission factor of $51 \mathrm{lb} / 10^{12} \mathrm{Btu}$. Emission factors for Mn ranged from 2.6 to $30 \mathrm{lb} / 10^{12} \mathrm{Btu}$, while values for Ni ranged from ND $<0.3$ to $40 \mathrm{lb} / 10^{12} \mathrm{Btu}$.

For eight ( $\mathrm{Sb}, \mathrm{As}, \mathrm{Cr}, \mathrm{Co}, \mathrm{Cu}, \mathrm{Pb}, \mathrm{Mo}$, and $\mathrm{V}$ ) of the 13 trace elements, the highest mean emission factors were reported for one or more sites that represented an ESP alone for emissions control: the Baldwin, Cardinal, or Niles Stations. The Baldwin Station, with a low SCA (180) ESP (95.6\%-98.1\% particulate control), reported the highest mean emission factors for six $(\mathrm{Cr}, \mathrm{Co}, \mathrm{Cu}, \mathrm{Pb}, \mathrm{Mo}$, and $\mathrm{V})$ of the 13 trace elements. The Coal Creek Station (ESP-wet FGD with $40 \%$ bypass) reported the highest mean emission factor for four $(\mathrm{Ba}, \mathrm{Be}, \mathrm{Cd}$, and $\mathrm{Mn})$ of the 13 trace elements. Plant Yates (ESP-JBR) reported the highest mean emission factor for Ni. However, the data variability was significant, and data quality (blank failure) was questionable in this case. The data indicate that emission control for these trace elements is directly related to the overall particulate control for the individual process configuration. 
Based on fuel feed rates ( 37 to 560 tons/hr) and analyses (6230 to $12,200 \mathrm{Btu} / \mathrm{lb}$ ) reported for the nine process configurations and an overall average capacity factor of 0.7 , the estimated annual combined emission rates for the nine nonvolatile trace element $\mathrm{HAPs}(\mathrm{Sb}, \mathrm{As}, \mathrm{Be}, \mathrm{Cd}, \mathrm{Cr}, \mathrm{Co}, \mathrm{Pb}, \mathrm{Mn}$ and $\mathrm{Ni}$ ) ranged from 0.04 to 2.98 tons/yr with an average emission rate for the nine process configurations of 0.62 tons/yr.

Adding $\mathrm{Hg}$ and Se to this group results in estimated trace element HAP emissions ranging from 0.06 to 5.65 tons/yr with an average emission rate for the nine process configurations of 1.5 tons/yr. Obviously, these values are only estimates, and any changes to the values used for coal feed rate, fuel heating value, or capacity factor can dramatically influence the estimated emission rates.

Penetration data also demonstrate a significant degree of variability and range of control for specific trace elements and individual emission control technologies. Emission control for these 13 trace elements was $>90 \%(<10 \%$ penetration) for all nine process configurations based on plant coal data, representing a wide range of firing configurations, fuel types, and emission control technologies. Particulate control alone (ESP or FF) limited trace element penetration to $<5 \%$ ( $>95 \%$ control) for ten ( $\mathrm{Sb}, \mathrm{Ba}, \mathrm{Be}, \mathrm{Cr}, \mathrm{Co}, \mathrm{Cu}, \mathrm{Pb}, \mathrm{Mn}, \mathrm{Ni}$, and $\mathrm{V}$ ) of the 13 trace elements. The three exceptions were $\mathrm{As}, \mathrm{Cd}$, and Mo. A combination of particulate control and SDA or wet FGD demonstrated $>99 \%$ trace element control for eight $(\mathrm{Sb}, \mathrm{As}, \mathrm{Ba}, \mathrm{Co}, \mathrm{Cu}$, $\mathrm{Pb}, \mathrm{Mn}$, and $\mathrm{V}$ ) of the 13 trace elements. Exceptions ( $\mathrm{Be}, \mathrm{Cd}, \mathrm{Cr}$, and $\mathrm{Ni}$ ) were noted for the Coal Creek Station (ESP-wet FGD with $40 \%$ bypass), Plant Yates (Cd and Ni, ESP-JBR), and the SNOX process (Mo) at the Niles Station. The exceptions noted for the Coal Creek Station appear to be the result of high reported nondetect concentrations for $\mathrm{Be}$ (ND $<2.27 \mu \mathrm{g} / \mathrm{Nm}^{3}$ ) and $\mathrm{Cd}$ (ND $<4.3 \mu \mathrm{g} / \mathrm{Nm}^{3}$ ), poor data quality for $\mathrm{Be}, \mathrm{Cd}, \mathrm{Cr}$, and $\mathrm{Ni}$ (failed blanks), and significant data variability for $\mathrm{Cr}$ and Ni. The exceptions noted for Plant Yates were a result of data variability and questionable data quality (failed spike recovery for $\mathrm{Cd}$ and failed blank for $\mathrm{Ni}$ ). The one exception noted for the SNOX process (Mo) appears to be the result of questionable data quality based on a failed blank. 
Emission control (percent penetration) based on round-robin coal data are comparable with a few exceptions. Twelve of the 13 elements resulted in $>90 \%$ control. Cadmium was the exception, with three sites having penetration values in the $10 \%$ to $40 \%$ range $(90 \%$ to $60 \%$ control). Nickel penetration for Coal Creek Station was $<1 \%$ ( $>99 \%$ control). The control of trace element emissions will never exceed the level of overall particulate control observed. Thus the DOE Phase I data generally demonstrate that the emission of these 13 trace elements is effectively controlled $(>98 \%)$ by highly efficient particulate control technology or combinations of technologies (ESP-wet FGD or SDA-FF) currently being used by the U.S. utility industry.

\subsection{Organic Emission Factors}

This discussion of organic emissions will focus on results obtained from samples at the stack. Four organic compound class samples were collected at each process configuration, including 1) aldehydes and ketones, 2) VOCs, 3) SVOCs and PAHs, and 4) chlorinated dioxins and furans. Each site contractor used standard EPA methods for collection and analysis (with some minor modifications in some cases) as described in the individual contractors' reports (11-19).

The organic results were quite variable for a variety of reasons, including low concentrations of target organics (requiring analyses to be performed very near detection limits), inadequate standard methods for flue gas sample collection and analyses of aldehydes and ketones, high blanks, and poor spike and surrogate recoveries. Because of the generally low concentrations found, the potential for errors in emission factors are very high for most species. Since there were sampling and analytical problems with the low concentrations encountered, the results summarized in this report should be viewed only as a representative range of potential emissions and should not be used for any projections requiring quantitative data.

The primary screening criteria used by the EERC to evaluate the organic emission data included results from blank samples, spike and audit samples, and DL for individual species. These $\mathrm{QA} / \mathrm{QC}$ criteria were the basis for qualifying organic 
emission data for inclusion in this summary report. Data that did not pass these criteria were determined to be indefensible for the purpose of quantifying and/or regulating organic emissions.

In order for a blank sample to pass the QA/OC criteria, the sample analysis must have indicated a nondetect or the presence of no more than $25 \%$ of the mean value of that organic species detected in the actual field sample. For the purpose of gathering reliable organic emission data, data recovered in an area where high blanks were noted were not considered conclusive and were interpreted as artificially high concentrations. Failure of blanks accounted for nearly all of the rejected data.

Spike and audit sample recoveries of $20 \%-150 \%$ were required for organic samples to pass the EERC QA/OC criteria. (It should be noted that the EERC considers a range of $70 \%$ to $125 \%$ to be necessary; however, the broader range was applied to conform with acceptance criteria used by most contractors based on EPA methods.) Data above the DL of the analytical method used is another criterion for data validation. The reporting of data below the $\mathrm{DL}$ or the reporting of nondetects as one-half the DL is not valid and could lead to future misuse or misunderstanding of the data. In cases where all values are nondetects, results should be reported as $N D<D L=x \cdot x x$.

Because of the problems encountered with blank and spiked samples during Phase I activities, all contractors should be required to pass round-robin evaluation for each organic analysis method, including successful generation of low blank values (including all relevant collection devices and analytical steps) and successful performance on blind audit samples prepared by a single organization prior to initiation of Phase II field activities. Each contractor should supply the sample collection devices for the audit samples.

Some of the special topic areas of the Phase I field sampling effort addressed organic emission issues. These included 1) PSDS; 2) determination of chlorinated dioxin-furan concentrations across ESPs; 3) vapor-particle distribution of SVOCs, PAHs, and chlorinated dioxins and furans; 4) comparison of canister and sorbent 
techniques for VOCs, and 5) organic concentrations in water samples. Key results for some of these issues are discussed in Section 4.6.

Tables summarizing the organic emission data, which passed QA/OC criteria, are contained in Appendix B. The remainder of this section discusses and presents emission data concerning specific organic compound classes, including aldehydes and ketones, VOCs, SVOCs and PAHs, and chlorinated dioxins and furans.

\subsubsection{Aldehydes and Ketones}

The majority of contractors had severe problems performing the aldehyde and ketone analyses, indicating significant shortcomings in the collection and analysis methods. In most cases, the concentrations found in laboratory and field blanks were similar to the concentrations reported for the stack samples. Several contractors also failed to achieve reasonable results for surrogate spike and audit sample analyses. Because of the overall poor quality of these results, the data for six of the nine process configurations were found to be highly unreliable and were not reported in this document based on any or all of the following failure criteria (listed in order of decreasing occurrence): 1) blank values $>25 \%$ of the sample value (typically the range was about $50 \%$ to $>150 \%$, 2) surrogate and/or audit sample recoveries greatly outside acceptance criteria, and 3) reported values very near detection limits (typically $<2 \times D L$ ).

The results from Coal Creek Station, Niles Station, and the SNOX process generally passed acceptance criteria; these are summarized in Tables 4-12 and B-1 (Appendix B). These results show that stack emissions of aldehydes are generally quite low, ranging from $<2$ to $41 \mathrm{lb} / 10^{12}$ Btu for acrolein to 67 to $390 \mathrm{lb} / 10^{12}$ Btu for acetaldehyde. Also note that the concentrations of aldehydes found in the stacks were typically within an order of magnitude of the ambient air concentrations (Table 4-12) reported by EPA (36). In fact, median ambient air concentrations generally fell within the reported stack concentration ranges measured, indicating that coal-fired power plants are not significant sources of these organic species. Based on these data and the inadequacy of the standard methods, aldehyde determination should be eliminated from Phase II field sampling activities. 
sampling devices used were clean or not. Surrogate and audit recoveries were acceptable, so the major reason for rejecting results was the occurrence of high (and often spurious) blanks. Quantitative VOC results were also suspect because concentrations reported were often very near detection limits. VOC results are summarized in Table 4-12, with more complete data for each site presented in Appendix B (Tables B-2 to B-9).

In general, the VOC species found in the stack emissions were present in quite low concentrations, usually in concentrations similar to those reported by the EPA to be found in ambient air, as summarized in Table 4-12. Of the 12 VoCs listed in Table 4-12, only two had median stack concentrations that exceeded the median ambient air concentration reported: benzene and chloromethane. The median stack concentrations for benzene and chloromethane were 1.67 and 50 times the median ambient air concentration (10 versus 6 and 75 versus $1.5 \mu \mathrm{g} / \mathrm{Nm}^{3}$, respectively). Several of the species (e.g., benzene and alkylbenzenes) are chemically quite reasonable emissions for coal-fired systems, while the presence of many of the reported species seems unlikely on a chemical basis le.g., common halogenated solvents). However, such species are reported in ambient air, making air inleakage a possible source. If Phase II or other future field sampling activities include VOCs, sampling should be limited to the stack and a site representing ambient air conditions around the plant. All samples should be collected and analyzed in quadruplicate. The sampling should be performed during representative operating conditions and does not need to encompass variations in plant operating conditions.

\subsubsection{Semivolatile Organic. Compounds and Polycyclic Aromatic Hydrocarbons}

The SVOC and PAH data appeared reliable, although some contractors had severe blank problems, which invalidated their results. In addition, surrogate and audit sample recoveries were sometimes out of the required range (e.g., $20 \%$ to $150 \%$ recovery), although this failure was not as frequent as the occurrence of high blanks. The results of the SVOC and PAH analyses are summarized in Table 4-12, with a more detailed summary given in Appendix B (Tables B-10 to B-15). 
Most of the SVOCs and PAHs were found at quite low concentrations, often near the detection limit. Several species seem very likely to be emitted from coalfired systems (e.g., phenols and all of the PAHs), while some reported species seem highly unlikely. Phthalate esters, which are present in ambient air, are more likely to be the result of a sample-handling problem than a true sample species. In any case, the concentrations of many SVOCs and PAHs are relatively low, as shown in Table 412. As with the VOCs, the SVOCs and PAHs reported were frequently found at concentrations near the $\mathrm{DL}$; therefore, the emission factors are to be considered only as estimations of the possible range. Species included in the EPA National Ambient VOC database that were reported for some of the power stations include phenol, methylphenols (cresols), naphthalene, and methylnaphthalene. As shown in Table 4-12, these species were present in the stacks at concentrations comparable to ambient concentrations. Although comparative ambient data for other SVOC and PAHs were not available, their concentrations are similar to (or lower than) the phenols and naphthalene. Therefore, it seems reasonable that the concentrations of the other species summarized in Table 4-12 are not highly significant compared to other sources (e.g., diesel exhaust). If Phase II or other future field sampling activities include SVOCs, sampling should be limited to the stack and a site representing ambient air conditions around the plant. Analyses should include phenol and alkylphenols (which were not included by several contractors in the Phase I study), as well as PAHs. All samples should be collected and analyzed in quadruplicate. The sampling should be performed during representative operating conditions and does not need to encompass variations in plant operating conditions.

\subsubsection{Chlorinated Dioxins and Furans}

Chlorinated dioxins and furans were analyzed at much lower concentrations $\left(\mathrm{pg} / \mathrm{Nm}^{3}\right)$ than the other species (e.g., SVOCs and PAHs). A few spurious detections were reported by some of the contractors; however, the reported concentrations were generally at DL values or invalidated by blank detections at similar concentrations. Since the majority of contractors reported reasonable results for spike recoveries, the data are best interpreted as indicating that no chlorinated dioxins or furans were present at levels above the detection limits. Therefore, concentrations of individual 
chlorinated dioxins and furans were typically less than 1 to $10 \mathrm{pg} / \mathrm{Nm}^{3}$ (depending on the detection limit for individual species by each contractor). Similarly, emission factors were typically $<3 \times 10^{-6}$ to $<6 \times 10^{-6} \mathrm{lb} / 10^{12}$ Btu (again, the lower limits depend on the analytical detection limits). These emission factors should be viewed as upper limits only and indicate that chlorinated dioxin and furan emissions are not significant from the coal-fired power plants included in this study. If Phase II or other future field sampling activities include chlorinated dioxins and furans, sampling should be limited to the stack and a site representing ambient air conditions around the plant in a manner similar to that discussed for VOCs, SVOCs, and PAHs.

\subsection{RADIONUCLIDE EMISSION FACTORS}

Radionuclides are listed generically as a CAAA HAP. All of the contractors provided radionuclide data, but the selection of radionuclides for which results were reported varied greatly among contractors. Results for $\mathrm{Pb}-210, \mathrm{Ra}-226$, and U-235 were reported for all nine process configurations. Results for Ra-228, Th-230, and U-234 were reported for all sites except Plant Yates. Results for Po-210, Pb-211, $\mathrm{Pb}-212$, Th-228, Th-229, Th-232, Th-234, and U-238 were reported for three to five sites each.

The results of radionuclide analyses for coal and stack gas samples, including emission factors and control device efficiency, are presented in Appendix C, Tables C-1 to C-14. Many contractors analyzed a variety of process stream samples for radionuclide content, but those data are beyond the scope of this report summarizing stack emissions. The coal, stack gas, and emission factor results are summarized in Table 4-13. Pb-210, Pb-212, Po-210, Ra-226, Ra-228, Th-228, Th-230, Th-232, Th-234, U-234, U-235, and U-238 were reported at detectable levels in at least some of the feed coal samples. The average levels at which these radionuclides were reported did not exceed $1: 5 \mathrm{pCi} / \mathrm{g}$. Overall, the range of detected radionuclide values in the feed coal samples was 0.02 to $7.3 \mathrm{pCi} / \mathrm{g}$. Th-230 had the highest average feed coal value detected $(2.1 \mathrm{pCi} / \mathrm{g})$, followed by Th-234 $(1.5 \mathrm{pCi} / \mathrm{g})$ and $\mathrm{Pb}-210$ $(1.3 \mathrm{pCi} / \mathrm{g})$. Many contractors reported numerous nondetect radionuclide values. 
Table 4-13. Summary of Radionuclide Results for Nine Coal-Fired Utility Process Configurations. (For the average values listed, corresponding ranges are given in parentheses if the average represents more than one value.)

\begin{tabular}{|c|c|c|c|c|c|c|c|c|}
\hline \multirow[b]{2}{*}{ Radionuclide } & \multirow[b]{2}{*}{$\begin{array}{l}\text { Number of } \\
\text { Plants } \\
\text { Reporting } \\
\text { Results }\end{array}$} & \multicolumn{2}{|c|}{ Coal Concentration } & \multicolumn{2}{|c|}{ Stack Concentration } & \multicolumn{3}{|c|}{ Emission Factor } \\
\hline & & \begin{tabular}{|l|} 
Number of \\
Plants \\
Reporting \\
Detectable \\
Values \\
\end{tabular} & $\begin{array}{l}\text { Average and } \\
\text { Range of Mean } \\
\text { Values, } \\
\mathrm{pCi} / \mathrm{g} \\
\end{array}$ & \begin{tabular}{|l} 
Number of \\
Plants \\
Reporting \\
Detectable \\
Values \\
\end{tabular} & $\begin{array}{l}\text { Average of } \\
\text { Mean } \\
\text { Values, } \\
\mathrm{pCi} / \mathrm{Nm}^{3} \\
\end{array}$ & $\begin{array}{l}\text { Average of } \\
\text { Mean Values, } \\
\mathrm{lb} / 10^{12} \mathrm{Btu}\end{array}$ & $\begin{array}{l}\text { Average of } \\
\text { Mean } \\
\text { Values, } \\
\mathrm{pCi} / 10^{12} \mathrm{Btu} \\
\end{array}$ & $\begin{array}{l}\text { Average of Control } \\
\text { Device Removal }(\%) \text { for } \\
\text { Plants with Detectable } \\
\text { Emission Factors }\end{array}$ \\
\hline $\mathrm{Pb}-210$ & 9 & 8 & $\begin{array}{c}1.3 \\
(0.52 \text { to } 2.0)\end{array}$ & 1 & - & $2.1 \times 10^{-6}$ & $7.2 \times 10^{10}$ & 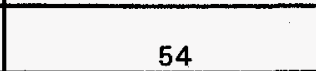 \\
\hline $\mathrm{Pb}-211$ & 3 & 0 & - & 0 & - & - & - & - \\
\hline $\mathrm{Pb}-212$ & 4 & 4 & $\begin{array}{c}0.26 \\
\langle 0.20 \text { to } 0.35\}\end{array}$ & 0 & - & - & - & $N A^{b}$ \\
\hline Po-210 & 4 & 4 & $\begin{array}{c}0.22 \\
(0.12 \text { to } 0.3)\end{array}$ & 1 & 2.7 & $3.9 \times 10^{-10}$ & $8.0 \times 10^{8}$ & 94 \\
\hline Ra-226 & 9 & 9 & $\begin{array}{c}0.54 \\
(0.2 \text { to } 1.2)\end{array}$ & 1 & 0.40 & $3.2 \times 10^{-7}$ & $1.4 \times 10^{8}$ & 98 \\
\hline $\mathrm{Ra}-228$ & 8 & 5 & $\begin{array}{c}0.79 \\
(0.40 \text { to } 1.9)\end{array}$ & 0 & - & encon & - & - \\
\hline Th-228 & 4 & 4 & $\begin{array}{c}0.34 \\
(0.17 \text { to } 0.5)\end{array}$ & 0 & - & - & - & - \\
\hline Th-229 & 3 & 0 & - & 0 & - & - & - & - \\
\hline Th-230 & 8 & 5 & $\begin{array}{c}2.1 \\
(0.25 \text { to } 7.3)\end{array}$ & 1 & 7.8 & $3.3 \times 10^{-4}$ & $3.1 \times 10^{9}$ & 73 \\
\hline Th-232 & 4 & 4 & $\begin{array}{c}0.26 \\
(0.17 \text { to } 0.37)\end{array}$ & 0 & - & 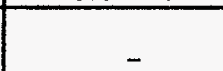 & 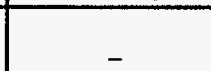 & - \\
\hline Th-234 & 4 & 3 & $\begin{array}{c}1.5 \\
(0.67 \text { to } 2.8)\end{array}$ & 0 & - & - & - & NA \\
\hline U-234 & 8 & 4 & $\begin{array}{c}0.63 \\
(0.07 \text { to } 1.1)\end{array}$ & 1 & 1.4 & $1.6 \times 10^{-4}$ & $4.5 \times 10^{8}$ & 97 \\
\hline U-235 & 9 & 5 & $\begin{array}{c}0.10 \\
(0.02 \text { to } 0.17)\end{array}$ & 1 & 24 & 8.2 & $8.1 \times 10^{9}$ & 51 \\
\hline U-238 & 5 & 5 & $\begin{array}{c}1.19 \\
(0.2 \text { to } 1.0)\end{array}$ & 2 & $1.8^{\mathrm{c}}$ & $\begin{array}{c}312 \\
(4.0 \text { to } 620)\end{array}$ & $\begin{array}{c}4.7 \times 10^{10} \\
\left(5.5 \times 10^{10} \text { to }\right. \\
\left.7.2 \times 10^{10}\right)\end{array}$ & $\begin{array}{c}74 \\
(52 \text { to } 96)\end{array}$ \\
\hline
\end{tabular}

Value of $210 \mathrm{pCi} / \mathrm{dscm} @ 3 \% \mathrm{O}_{2}$ reported for Cardinal Station.

"Not available.

c Cardinal Station value of $270 \mathrm{pCi} / \mathrm{dscm} @ 3 \% \mathrm{O}_{2}$ not included in average. 
Radionuclides were found in much lower concentrations in the stack gas particulate samples. U-238 was reported at detectable levels at two plants. $\mathrm{Pb}-210$, Po-210, Ra-226, Th-230, U-234, and U-235 were each reported as detected at one site. The highest average detected value for a stack gas sample was $24 \mathrm{pCi} / \mathrm{Nm}^{3}$ for $\mathrm{U}-235$, followed by $7.8 \mathrm{pCi} / \mathrm{Nm}^{3}$ for Th-230.

The calculated emission factor values for radionuclides were also low. Average emission factors, shown in Table 4-13, covered a broad range. In units of mass, average emission factors ranged from $3.9 \times 10^{-10} \mathrm{lb} / 10^{12} \mathrm{Btu}$ for Po-210 to $312 \mathrm{lb} / 10^{12} \mathrm{Btu}$ for $\mathrm{U}-238$. Most of the average emission factors were in the range of $10^{-7}$ to $10^{-4} \mathrm{lb} / 10^{12}$ Btu. In units of activity, average emission factors ranged from $1.4 \times 10^{8} \mathrm{pCi} / 10^{12} \mathrm{Btu}$ for Ra-226 to $7.2 \times 10^{10} \mathrm{pCi} / 10^{12} \mathrm{Btu}$ for $\mathrm{Pb}-210$. Most of the average emission factors were in the range of $10^{8}$ to $10^{9} \mathrm{pCi} / 10^{12}$ Btu.

Average control device removal ranged from $51 \%$ for $\mathrm{U}-235$ to $98 \%$ for Ra-226, but these values should be interpreted with caution because of the reporting of nondetect activity data and their use in emission factor calculations. In general, however, the radionuclide data set suggests that radionuclide emissions are very low for the Phase I coal-fired power plants.

\subsection{Acid Gases - Halogen Emission Factors}

Phase I contractors performed halogen and acid gas sampling for both particulate- and vapor-phase forms. Figures 4-23 and 4-24 show the range of emission factors and percent penetration. Total chlorine $\left(\mathrm{HCl}+\mathrm{Cl}_{2}\right)$ emissions ranged from ND $<176$ to $132,000 \mathrm{lb} / 10^{12} \mathrm{Btu}$, resulting in an estimated annual emission of 1.6 to 1645 tons/yr. Total fluorine $\left(H F+F_{2}\right.$ ) emissions ranged from ND $<92$ to $12,770 \mathrm{lb} / 10^{12} \mathrm{Btu}$. Emission factors for $\mathrm{HCN}$ ranged from ND $<2.2$ to $180 \mathrm{lb} / 10^{12} \mathrm{Btu}$. Where performed, QA/OC for halogen and acid gas sampling was satisfactory; however, the averaged data showed large standard deviations. 
Vapor-phase halogens were collected as $\mathrm{HCl}$ and $\mathrm{HF}$ at all but three sites: Boswell Energy Center (FF), Cardinal Station (ESP), and Baldwin Station (ESP), where they were speciated into $\mathrm{HCl}$ and chlorine gas $\left(\mathrm{Cl}_{2}\right)$ and $\mathrm{HF}$ and fluorine gas $\left(\mathrm{F}_{2}\right)$. Speciation at Cardinal Station showed $\mathrm{Cl}_{2}$ as approximately $6.3 \%$ of total chlorine and $\mathrm{F}_{2}$ as approximately $85 \%$ of total fluorine. Baldwin Station showed $\mathrm{Cl}_{2}$ as approximately $5.5 \%$ of total chlorine and no $F_{2}$. Boswell Energy Center showed the largest degree of speciation with $\mathrm{Cl}_{2}$ as $45 \%$ of total chlorine and $\mathrm{F}_{2}$ as $25 \%$ of total fluorine. These percentages were relatively consistent at the ESP or FF inlet through the stack. While $\mathrm{HCl}, \mathrm{Cl}_{2}$, and $\mathrm{HF}$ are on the list of $189 \mathrm{HAPs}, \mathrm{F}_{2}$ is not. $\mathrm{HBr}$ and $\mathrm{Br}_{2}$ were sampled and analyzed at Baldwin Station and Boswell Energy Center, but neither was detected at either site.

Since the existence of $\mathrm{Cl}_{2}$ as well as $\mathrm{F}_{2}$ is debatable in combustion flue gas, it is important to remember that in the EPA Method 26A sampling technique, the

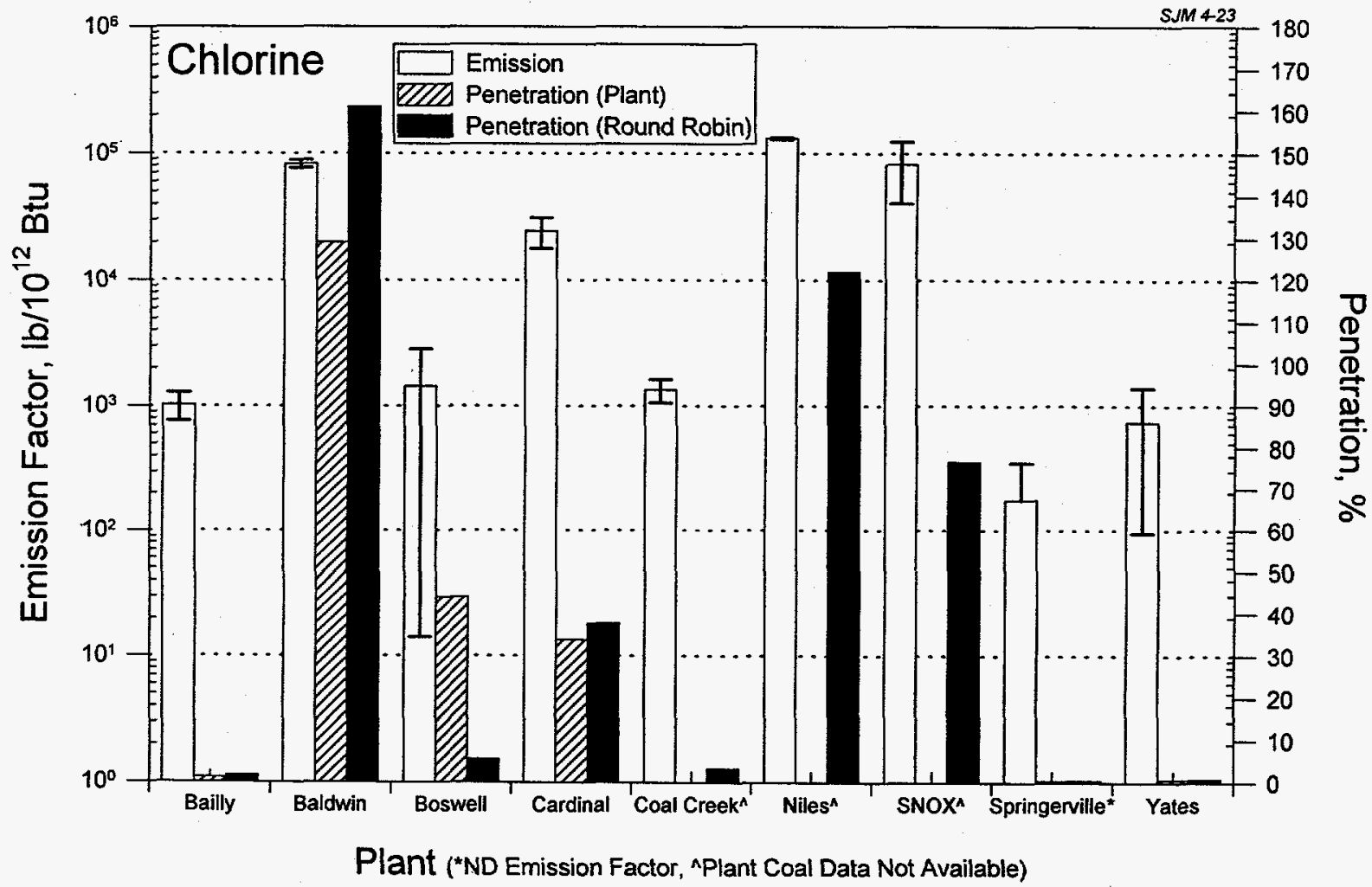

Figure 4-23. Emission Factor and Penetration Data for Total Chlorine $\left(\mathrm{HCl}+\mathrm{Cl}_{2}\right)$. 


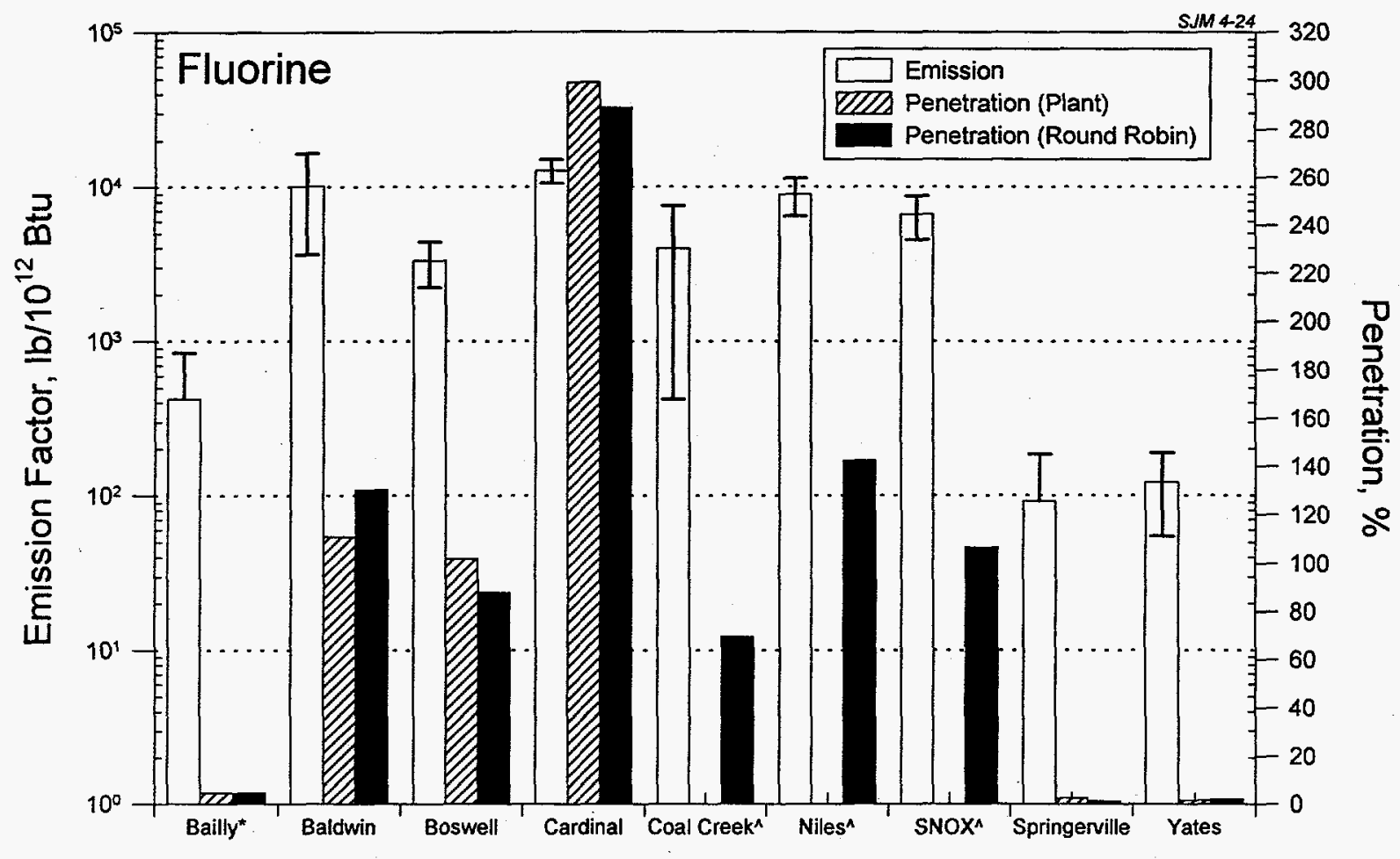

Plant ("ND Emission Factor, ^Plant Coal Data Not Available)

Figure 4-24. Emission Factor and Penetration Data for Total Fluorine $\left(H F+F_{2}\right)$.

chlorine captured in the dilute $\mathrm{H}_{2} \mathrm{SO}_{4}$ impingers existed as $\mathrm{HCl}$, while the chlorine captured in the dilute $\mathrm{NaOH}$ impingers existed as $\mathrm{Cl}_{2}$ and/or other volatile chlorine containing compounds. To further investigate the halogen speciation results found in Phase I, tests were conducted at CONSOL INC (16). Data from these tests showed approximately $3 \%$ of the total chlorine in the dilute $\mathrm{NaOH}$ impinger of which $<1 \%$ could be attributable to $\mathrm{HCl}$ carryover from the dilute $\mathrm{H}_{2} \mathrm{SO}_{4}$ impingers.

As expected, removal of $\mathrm{HCl}, \mathrm{HF}$, and $\mathrm{HCN}$ did not occur across ESPs except for Cardinal Station, which showed approximately $25 \%$ removal for $\mathrm{HCl}$. Rather, nearly all data indicated equal or higher concentrations of acid gases at the ESP outlets than at the ESP inlets. Overall negative removals were usually calculated from two negative and one positive removal test days. The FF at Niles Station (part of the SNOX process) showed an overall negative removal of acid gases. Worthy of note, the FF at Boswell Energy Center showed removals of $\mathrm{HCl}(35 \%-65 \%)$, 
$\mathrm{Cl}_{2}(60 \%-97 \%), \mathrm{F}_{2}(53 \%-72 \%), \mathrm{HCN}(35 \%)$, and $\mathrm{HF}$ (negative). Acid gas removal across a FF is possible if reactions or adsorption occur in the filter dust cake, but conclusions cannot be drawn from the data at one plant. Data from the FF at Springerville Station were invalidated during sampling.

Also as expected, removal of $\mathrm{HCl}, \mathrm{HF}$, and $\mathrm{HCN}$ were significant across $\mathrm{FGD}$ systems and highly effective across advanced FGD systems. The AFGD system at Bailly Station removed $99 \%$ and $96 \%$ of $\mathrm{HCl}$ and $\mathrm{HF}$, respectively, and $\mathrm{HCN}$ to a lesser extent. The JBR at Plant Yates (which had the highest coal chlorine concentration) removed $>99 \%$ of the acid gases. Sampling and analytical problems preclude conclusions for the SDA at Springerville Station. The Coal Creek Station wet FGD (with $40 \%$ FGD bypass) removed $51 \%$ of $\mathrm{HCl}, 44 \%$ of $\mathrm{HCN}$, but only $2.3 \%$ of HF. The SNOX process (an acid condensation method) removed $32 \%-52 \%$ of $\mathrm{HCl}$, approximately $52 \% \mathrm{HCN}$, and $1 \%-12 \%$ of $\mathrm{HF}$. Higher operating temperatures $\left(200^{\circ} \mathrm{F}\right)$ and the absence of alkali for reaction render the SNOX process less effective than other AFGD systems for acid gas-halogen removal.

Particulate phase $\mathrm{Cl}^{-}, \mathrm{F}^{-}$, and $\mathrm{CN}^{-}$were measured below $\mathrm{DL}$ for most of the sites and contributed to $<5 \%$ of the total halogen concentration for the remaining sites. Fabric filter and ESP removal for $\mathrm{Cl}^{-}$was $>90 \%$ to $99 \%$ for all sites, whereas $\mathrm{F}^{-}$ showed greater variability, 55\%-99\% removal. The ESP at Coal Creek Station appeared to increase $\mathrm{F}^{-}$concentration levels, but given the high degree of data variability, the data are largely inconclusive. Particulate cyanide, where measured above the $\mathrm{DL}$, showed a range of removal between negative and $35 \%$. The relative removal efficiencies for $\mathrm{Cl}^{-}, \mathrm{F}^{-}$, and $\mathrm{CN}^{-}$compounds are very dependent upon the ESP removal efficiency for the given compound particle size where $\mathrm{Cl}>\mathrm{F}>\mathrm{CN}$.

Using the round-robin coal data, the highest chlorine coal concentrations were at Plant Yates and Niles/SNOX Station, 1200 and $1400 \mu \mathrm{g} / \mathrm{g}$ respectively, and the lowest were at Bailly, Springerville, and Coal Creek Stations (300 to $400 \mu \mathrm{g} / \mathrm{g}$ ). The remaining sites ranged from 640 to $850 \mu \mathrm{g} / \mathrm{g}$. Coal fluorine round-robin data indicated that Boswell Energy Center and Cardinal and Coal Creek Stations had the lowest 
concentrations (44 to $58 \mu \mathrm{g} / \mathrm{g}$ ). The remaining sites ranged from 80 to $110 \mu \mathrm{g} / \mathrm{g} \mathrm{coal}$ fluorine concentrations, with Bailly Station at the high end.

Percent chlorine penetration was less than $2 \%$ for sites with advanced FGD systems (Bailly Station, Springerville Station, and Plant Yates). Both plant and roundrobin coal data are in excellent agreement. Cardinal Station plant and round-robin data were also in good agreement $(34 \%$ and $38 \%$ penetration). Large differences were observed between plant and round-robin percent chlorine penetration for Baldwin Station and Boswell Energy Center. Baldwin Station plant data showed over $130 \%$ penetration, although chlorine mass balances were reasonable $(105 \%$ to $133 \%)$, whereas the round-robin data showed $160 \%$ penetration. Boswell Energy Center plant data yielded $44 \%$ chlorine penetration, while the round-robin data yielded $5 \%$. Yet, the mass balances based on plant data for chlorine at Boswell Energy Center were low, between $20 \%$ and $50 \%$. Round-robin data for Niles Station showed chlorine penetration around $120 \%$, and the SNOX process showed $77 \%$ penetration. Plant data from Coal Creek and Niles/SNOX Stations were not available.

Based on control technology, the combined particulate and advanced FGD systems showed very little chlorine penetration including Coal Creek Station with $40 \%$ scrubber bypass. Two ESP sites (Baldwin and Niles Station) showed over $100 \%$ chlorine penetration, while Cardinal Station (ESP also) showed less than $50 \%$ chlorine penetration. Of two FF sites with acid gas-halogen data, one showed high penetration (77\%), while the averaged percent penetration (from the plant and roundrobin coal data) for the other FF was less than the best performing ESP. The SNOX process generally showed less penetration than the ESPs and more than the FFs.

Percent fluorine penetration across advanced FGD systems (Bailly Station, Springerville Station, and Plant Yates) was similar to chlorine. Both plant and roundrobin data showed less than $5 \%$ fluorine penetration. Though the remaining sites show good agreement between round-robin and plant data, fluorine penetration is greater than $100 \%$, ranging from $102 \%$ to $300 \%$, with the exception of Coal Creek Station. A likely source of error is the difficulty in obtaining accurate coal fluorine concentrations. Current analytical methods show a low bias for total fluorine. 
The trend for percent fluorine penetration based on control technologies is similar to chlorine. Sites with particulate control only and the SNOX process had very high fluorine penetration while combined particulate-wet FGD systems had $<5 \%$ fluorine penetration. Coal Creek Station (with $40 \%$ bypass) showed $70 \%$ fluorine penetration, although it showed $<5 \%$ chlorine penetration.

Total chlorine emissions alone represent a significantly large amount, $23 \%$ to $93 \%$, of total HAP emissions from the Phase $I$ sites. Since $F_{2}$ is not a HAP and speciation into $F_{2}$ and HF was not characterized at all sites, it is more difficult to measure the contribution of HF to total HAPs. Phase I data demonstrate that combined particulate-advanced FGD systems are very effective for controlling both particulate- and vapor-phase halogens and acid gases. Particulate control systems effectively remove particulate-phase halogens but are completely ineffective for vapor phase halogens.

\subsection{Discussion OF SPECIAL TOPICS}

\subsubsection{Plume-Simulating Dilution Sampling}

Sampling with dilution was conducted at the stack of four of the sites to simulate the cooling and dilution of the flue gas that occurs in the plume shortly after it exits the stack. Potential changes anticipated as a result of the cooling and dilution include condensation of vapor to the particulate phase for some species, adsorption of vapor-phase species onto particle surfaces, and changes in the chemical species of some elements. At two of the sites, the dilution was approximately 10 parts dry filtered air to 1 part flue gas. At the other two sites, the dilution was approximately 20 parts dilution gas (consisting of an $80 \%$ nitrogen and $20 \%$ oxygen mixture of pure gases) to 1 part flue gas. Comparative sampling was done with and without dilution at the stack-sampling location at all four sites. EPA Method 29 sampling was conducted to determine the effect of cooling and dilution on the particulate-vapor split of the trace elements. At two of the sites, impactor sampling was done to determine the effect of cooling and dilution on the particle-size distribution. Sampling 
for organic species was conducted to determine whether cooling and dilution would change the species detected and their relative concentrations.

Stack concentrations of most of the trace substances are very low, and achieving good precision among repeat measurements is already challenging. The difficulty is greatly amplified when the flue gas samples are diluted by a factor of 10 to 20 . For credible quantitative results, the PSDS method must be verified with rigorous $\mathrm{QA} / \mathrm{OC}$, including blank analyses to ensure that blank corrections are not a significant portion of the measured values. Measured concentrations of the trace elements in the stack flue gas for the diluted and undiluted samples must be in good agreement, and the variability of the repeat measurements must be smaller than any observed effect of the cooling and dilution. The measurements at all four sites, however, had poor precision and poor agreement between the diluted and undiluted samples. In some cases, the measured vapor-phase fraction of some elements was greater for the diluted sample compared to the undiluted sample, which is opposite from theoretical expectations and indicative of the poor data quality. Because of the data quality problems, no firm conclusions can be drawn from the plume dilution sampling results other than the demonstrated need for much more development of this sampling approach. Nevertheless, several of the main observations from the plume dilution sampling results should help focus future development efforts:

- For the Bailly Station, a significant shift from vapor to particulate phase as a result of dilution sampling was noted for $\mathrm{B}, \mathrm{Hg}$, and $\mathrm{Se}$. Whether homogeneous nucleation or heterogeneous nucleation occurred, whether the effect was adsorption on existing particulate matter, and whether the observed effect may also have been associated with a change in chemical species are not known.

- For the Coal Creek and Niles Stations, an increase in concentration for SVOC/PAH was noted as a result of dilution sampling. Even though the data are variable, the consistency of the trend from day to day for several species and the observation at both stations suggest that the effect is a result of the process rather than random variability. These data indicate that the compounds are being formed in the dilution and cooling process. 
- For the Coal Creek Station, a small shift in particle size from a mass median diameter (MMD) of $2.6 \mu \mathrm{m}$ with hot undiluted sampling to $2.0 \mu \mathrm{m}$ with dilution sampling was noted. The shift may have been caused by a combination of sampling losses for the larger particles and some generation of fine-particle mass because of a shift of some vapor-phase material to fine particles. For the Niles Station, the change was much more pronounced, the particle size shifting from a MMD of $2.9 \mu \mathrm{m}$ with hot, undiluted sampling to $0.1 \mu \mathrm{m}$ with dilution sampling. The shift was the result of a substantial weight gain on the impactor backup filter with dilution sampling. A significant shift like this would not be expected unless there was substantial condensation of a substance such as $\mathrm{SO}_{3}$ present in fairly high concentrations. However, the backup filters were apparently not analyzed, so the cause of this significant shift in MMD as a result of dilution sampling is not known. Battelle personnel (who conducted the sampling) believe the filter weight gain was real, but suspect that it was caused by some unknown sampling artifact.

\subsubsection{Trace Element Enrichment as a Function of Particle Size}

Sampling with cyclones and a backup filter was conducted at the inlet of the particulate control device at each plant to provide three particulate size fractions that were analyzed for trace element concentration. A comparison of the largest and smallest size fractions provides an indication of the potential for enrichment of trace element concentration with decreasing particle size. The cut point of the largest cyclone ranged from 8 to $10 \mu \mathrm{m}$, and the cut point of the smallest cyclone for seven of the nine process configurations ranged from 4 to $6 \mu \mathrm{m}$, but the cut point of the smallest cyclone was $1 \mu \mathrm{m}$ at two of the sites. Therefore, for seven of the sites, the comparison was between the fractions larger than about $10 \mu \mathrm{m}$ and smaller than about $5 \mu \mathrm{m}$, but for two of the sites the comparison was between the fractions larger than $10 \mu \mathrm{m}$ and smaller than $1 \mu \mathrm{m}$. These data are presented in Figure 4-25 as an enrichment factor for 16 elements. For most elements, all nine data points were available, but for some elements, such as $\mathrm{Hg}$, fewer data points were available. The greatest enrichment factor was observed for $\mathrm{Sb}, \mathrm{As}, \mathrm{Mo}$, and Se, which all had a median enrichment factor of approximately 5 . For three of these elements (Sb, Mo, 


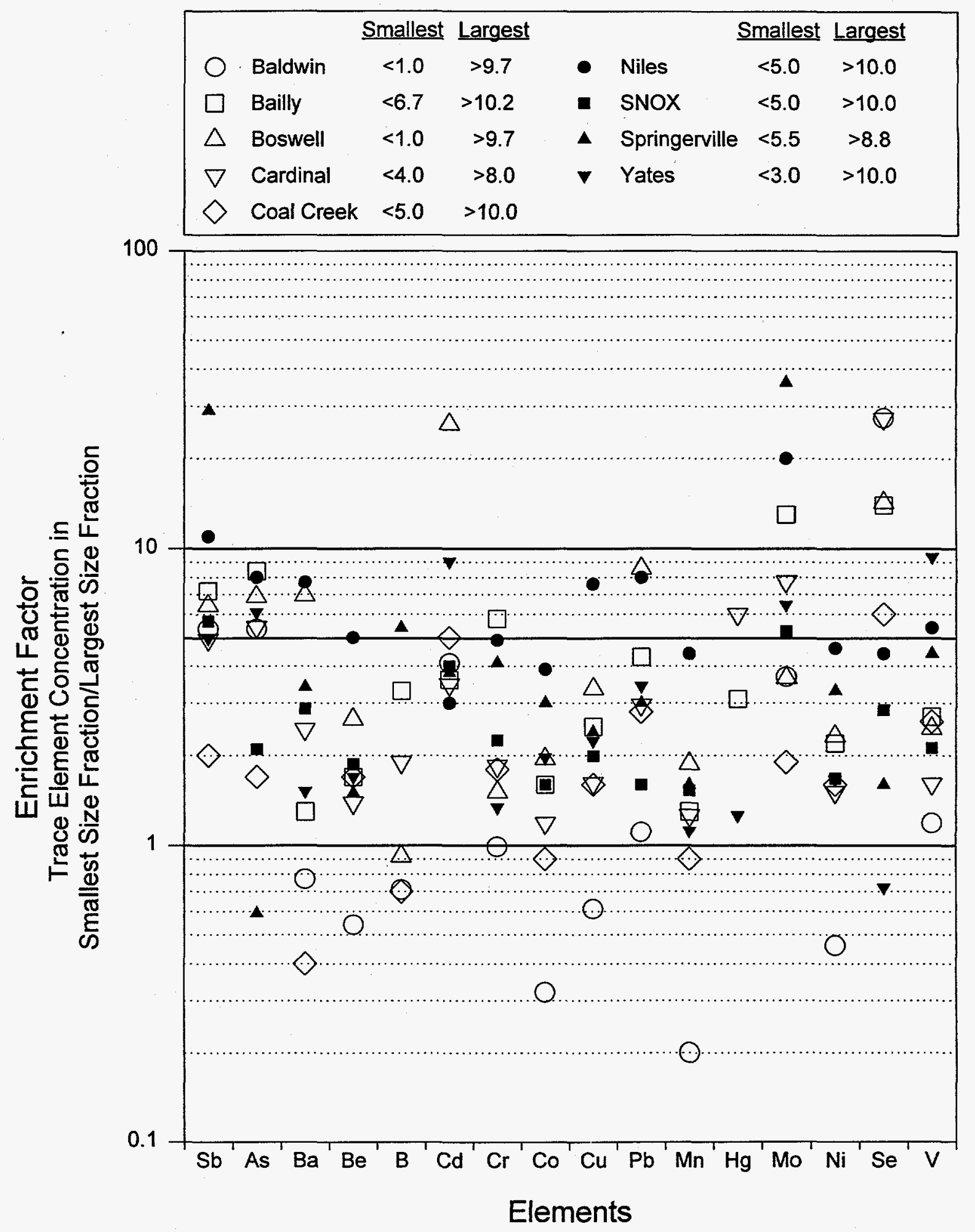

Figure 4-25. Trace Element Enrichment Factor, Comparison of Largest and Smallest Particle-Size Fractions. 
and Se), enrichment factor values greater than 10 were observed at two or more sites. Since these trace elements are among the most volatile that occur in the solid phase at the particulate control device inlet temperature, enrichment factors of greater than 10 are not surprising. If analyses of smaller size fractions were completed, the enrichment factors would be expected to be even higher. Because of data variability, firm conclusions on the enrichment potential for each element at each site cannot be drawn, but the data cumulatively suggest that significant enrichment with decreasing particle size is most likely to occur for $\mathrm{Sb}, \mathrm{As}, \mathrm{Mo}$, and Se. Because of this enrichment, trace element removal efficiency is likely to be lower than total particulate removal efficiency when a control device such as an ESP is used because collection efficiency deteriorates for smaller particles.

\subsubsection{Particle-Size Distributions of Stack Emissions and FF/ESP Hopper Ash}

Since certain health risks associated with trace element emissions depend on particle size, there is interest in determining the size distribution of particulate emissions. The particle-size distribution was measured at each of the sites at the inlet to the control device and at the stack. Under the high inlet dust-loading conditions, the most common measurement method was staged cyclones. For most of the inlet measurements, the dust was divided into only three fractions, so a well-defined distribution was not available. For some of the sites, reliable cyclone data were not available, but separate size analyses were conducted on ash samples from either the ESP or FF hoppers. At the stack, impactor data were available for most of the sites, with sufficient information to at least obtain the MMD, defined as the $50 \%$ point of the cumulative size distribution; in other words, $50 \%$ of the particulate mass is larger, and $50 \%$ of the particulate mass is smaller. Mass median diameter values at both the inlet and stack locations for each site are presented in Table 4-14. All particle diameter values are presented as aerodynamic diameter, defined as the equivalent diameter of a unit density sphere that has the same settling velocity as the actual particle at low Reynolds number. Since staged cyclones and impactors separate particles on the basis of their aerodynamic behavior for a variety of particle shapes and densities, it is the aerodynamic diameter that is actually determined. 
Table 4-14. Mass Median Aerodynamic Diameter at Control Device Inlet and Stack

\begin{tabular}{|c|c|c|c|c|c|c|c|c|c|}
\hline & $\begin{array}{l}\text { Coal } \\
\text { Creek }\end{array}$ & Bailly & Cardinal & Baldwin & Boswell & Niles & SNOX & Springerville & Yates \\
\hline $\begin{array}{l}\text { Inlet } \\
\text { Measurement } \\
\text { Method. }\end{array}$ & $\begin{array}{l}\text { Coulter } \\
\text { counter }\end{array}$ & Cyciones & & Malvern & $\begin{array}{l}\text { Cyclones/ } \\
\text { Malvern }\end{array}$ & & $\begin{array}{l}\text { Coulter } \\
\text { counter }\end{array}$ & Cyclones & $\begin{array}{l}\text { Cyclones/ } \\
\text { impactor }\end{array}$ \\
\hline Inlet MMD & 22 & 20 & $N A^{a}$ & $16^{b}$ & 14 & NA & 23 & 13 & $20^{b}$ \\
\hline $\begin{array}{l}\text { Stack } \\
\text { Measurement } \\
\text { Method }\end{array}$ & Impactor & Impactor & Impactor & Cyclones & & Impactor & Impactor & Impactor & Impactor \\
\hline Stack MMD & 2.5 & $0.55^{\circ}$ & 4.0 & 8.0 & NA & $2.9^{\circ}$ & 3.5 & 9.0 & 6.4 \\
\hline
\end{tabular}

a Results were not available or were of questionable data quality.

Sample taken from Field 1 and 2 ESP hopper ash.

${ }^{c}$ Likely outlet PSD influenced by acid condensation.

Aerodynamic diameter can be converted to geometric diameter assuming spherical particles and a known particle density.

The inlet MMDs ranged from 13 to $23 \mu \mathrm{m}$ which is typical of previous measurements of fly ash particle size for coal-fired boilers (37). Some size fractionation between the inlet and stack is expected because most control devices collect larger particles at a higher efficiency than smaller particles. However, the sizefractionating mechanisms for ESPs, FFs, and scrubbers are quite complex when cleaning-cycle effects are considered. Stack MMD values of 0.55 to $9.0 \mu \mathrm{m}$ represent a much broader range than the inlet MMDs, demonstrating complex and varying size-fractionating mechanisms. Two of the outlet MMD values may have been influenced by submicron acid condensation, which may significantly skew the stack particle-size distribution toward finer sizes. In all of these cases, the stack MMD was smaller than the inlet MMD, but the ratio of inlet to stack MMD ranged from 1.4 to 36 , which demonstrates that the particle size of the stack emission is likely to be highly system-specific. 


\subsubsection{Chromium Speciation and Sampling}

A key issue impacting the risk assessments for utility boilers is chromium speciation. Chromium may exist as the highly toxic hexavalent form and/or the less toxic trivalent form. In the recently promulgated EPA rules regulating the burning of hazardous waste in boilers and industrial furnaces, all chromium emissions to the atmosphere are considered to be hexavalent unless the facility in question performs stack tests specifically for hexavalent chromium. Studies to measure the amount of hexavalent chromium in stack gas emissions were carried out at two sites: Plant Yates (Radian) and Cardinal Station (EER).

The determination of hexavalent chromium in coal combustion flue gas is not a standardized technique. Both testing sites utilized EPA Method 0013 (Draft), the recirculating caustic solution method (38). It was noted in both sampling reports that although this test method is commonly used in incineration systems, it has not been tested for the high- $\mathrm{SO}_{2}$ content found in coal combustion gases. A discussion of the potential problems with the sampling technique is provided by Radian (14):

... $\mathrm{Cr}(\mathrm{VI})$ is stable in a strong alkaline solution $(\mathrm{pH}>9)$. But all combustion gas streams contain large amounts of $\mathrm{CO}_{2}(10 \%-20 \%)$, which is an acid gas, and serves to lower the $\mathrm{pH}$ of the impinger solution. As a result, the $\mathrm{pH}$ may dip lower than desirable during sampling, or the solution must be more alkaline than specified in the method or continually monitored. As a further complication, utility flue gas contains significant levels of $\mathrm{SO}_{2}(100 \mathrm{ppm}$ or more $) . \mathrm{SO}_{2}$ is also an acid gas but is a reductant as well. The impinger solution designed to absorb $\mathrm{Cr}(\mathrm{VI})$ also absorbs $\mathrm{CO}_{2}$ and $\mathrm{SO}_{2}$. The result of this is a lowered $\mathrm{pH}$ and a solution that contains an oxidant $[\mathrm{Cr}(\mathrm{VII})]$ and a reductant $\left(\mathrm{SO}_{2} / \mathrm{HSO}_{3}{ }^{-}\right)$. As the $\mathrm{pH}$ falls, the redox couple becomes more favorable, and any $\mathrm{Cr}(\mathrm{VI})$ present may be reduced by $\mathrm{SO}_{2} / \mathrm{HSO}_{3}{ }^{-}$and not detected as $\mathrm{Cr}(\mathrm{VI})$. 
The test method used by EER included a modification for the high $\mathrm{SO}_{2}$ content of the gas stream as recommended by Steinsberger et al. (39). In addition to the comments in the sampling reports, research at the EERC has demonstrated that care must be exercised in the alkaline preservation of chromium-containing solutions (40). The $\mathrm{pH}$ during preservation must remain well below 11.5 to prevent the possible formation of insoluble chromium-substituted calcium aluminate sulfate hydroxide hydrate (ettringite) phases. This can occur in the presence of calcium and aluminum and can be formed if fine particulates are present or in filtrates from ash and related materials.

The samples from EER were analyzed at RTI with ion chromatography in an ultratrace mode with a postcolumn reactor utilizing diphenylcarbazide. Samples were analyzed similarly by Radian without utilization of the ultratrace mode. The results from the sampling are shown in Table 4-15. It is noted that none of the values produced are usable in an emission factor calculation because of the large field blanks. Overall, the level of hexavalent chromium that could potentially be emitted from a system is small. The ratio of hexavalent chromium to total chromium (using blank corrected values) produces ratios of approximately $33 \%$ and $0.32 \%$ for Plant Yates and Cardinal Station, respectively. Thermochemical equilibrium predictions (41) have shown that only trace amounts of hexavalent chromium will form under stoichiometric conditions and that under very oxygen-rich conditions $(\mathrm{O} / \mathrm{C}>3)$, approximately $10 \%$ of the chromium can exist in the hexavalent form. Because of the consistency between the two different sampling sites where the field blanks are higher than the measured amounts, it is probable that interactions between the flue gas and solutions result in the reduction of any hexavalent chromium.

The data indicate that only minimal amounts of hexavalent chromium may be emitted from coal combustion systems. The EERC recommends that a thorough set of validation tests be performed on sampling and analytical protocols for chromium speciation. Once validated, sampling and analytical protocols can be applied to full-scale systems to document chromium speciation and to avoid the current EPA practice where all chromium emissions are considered to be hexavalent chromium. 
Table 4-15. Average Stack Concentrations, Field Blanks, and Emission Factors of Hexavalent Chromium

\begin{tabular}{|c|c|c|c|c|c|}
\hline $\begin{array}{l}\text { Sampling } \\
\text { Sites }\end{array}$ & $\begin{array}{c}\text { Field } \\
\text { Blank, } \\
\mu \mathrm{g} / \mathrm{Nm}^{3} \mathrm{a}\end{array}$ & $\begin{array}{c}\text { Stack Gas } \\
\text { Concentration } \\
\text { Measured, } \\
\mu \mathrm{g} / \mathrm{Nm}^{3}\end{array}$ & $\begin{array}{c}\text { Ratio: Field } \\
\text { Blank/Stack Gas } \\
\text { Concentration }\end{array}$ & $\begin{array}{c}\text { Corrected } \\
\text { Stack Gas } \\
\text { Concentration }\end{array}$ & $\begin{array}{l}\text { Emission } \\
\text { Factor, } \\
\mathrm{lb} / 10^{12} \mathrm{Btu}\end{array}$ \\
\hline $\begin{array}{l}\text { Yates } \\
\text { Cardinal }\end{array}$ & $\begin{array}{l}N^{c, d} \\
0.192\end{array}$ & $\begin{array}{c}\text { NR } \\
0.0988^{f}\end{array}$ & $\begin{array}{l}>1^{\mathrm{a}} \\
1.94\end{array}$ & $\begin{array}{c}\text { ND }<0.19 \\
0.0263\end{array}$ & $\begin{array}{l}N C^{e} \\
N C\end{array}$ \\
\hline $\begin{array}{ll}\text { a Field blank } \\
\text { b Values of } \\
\text { field blank } \\
\text { c In all cases } \\
\text { the correct } \\
\text { o Not report } \\
\text { \& Not calcula } \\
\text { Value calc }\end{array}$ & $\begin{array}{l}\text { concentration } \\
\text { field blank and } \\
\text { exceeded the } \\
\text {, the field bla } \\
\text { ted concentrat } \\
\text { ed. } \\
\text { ated; in all cas }\end{array}$ & $\begin{array}{l}\text { calculated assuming } \\
\text { I stack gas concentrat } \\
\text { value of the stack ga } \\
\text { nk exceeds the meast } \\
\text { ion. } \\
\text { es, field blanks excee } \\
\text { least one nondetect }\end{array}$ & $\begin{array}{l}\text { an average flow rate. } \\
\text { tion are not given, but it } \\
\text { is concentration. } \\
\text { ured stack concentration } \\
\text { d measured concentrati } \\
\text { value. }\end{array}$ & $\begin{array}{l}\text { as stated in the cor } \\
\text { nd thus the detecti }\end{array}$ & $\begin{array}{l}\text { or report that the } \\
\text { hit is listed for }\end{array}$ \\
\hline
\end{tabular}

\subsubsection{Dioxins-Furans Across ESPs}

Only one out of nine process configurations involved in an assessment of toxic emissions from coal-fired power plants reported relevant ESP inlet and outlet data for the determination of dioxin-furan concentrations across ESPs (19).

EER reported dioxin and furan data based on samples from the inlet of an ESP and at the stack (ESP outlet) for Cardinal Station Unit No. 1. Spurious detection of dioxin and furan congeners was reported based on these samples. According to the final report produced by EER, these results were considered questionable, since most compounds detected had high blank levels; all surrogate compound recoveries at the ESP inlet were outside the EER QA/OC limits; and the majority of the results reported had substantial portions of data attributable to nondetect data. Despite the data quality problems that affected most of the EER dioxin and furan emission data, ESP removal efficiency and penetration at the Cardinal Station were reported.

Essentially, the chlorinated dioxins and furans detected at the ESP inlet were at significantly lower concentrations than the other species (e.g., typically 6 orders of magnitude lower than SVOC and PAHs). All but two compounds (1-4,6-8-HpCDD [heptachloro dibenzo-p-dioxins] and OCDD [octachloro dibenzo- $p$-dioxins]) were above 
the detection limits at the ESP inlet, and their reported concentrations were $1.22 \times$ $10^{-6}$ and $1.41 \times 10^{-5} \mathrm{lb} / 10^{12} \mathrm{Btu}$, respectively. However, the data quality of these reported values may be suspect because the high blank values and surrogate sample recoveries were outside the QC limits (maximum blank ratios ranged from $99.3 \%$ of OCDD emissions to $360 \%$ of $1-4,6-8-\mathrm{HpCDD}$ emissions). Note that maximum blank ratios are defined as the ratio of the highest blank value to the uncorrected sample value. If the maximum blank ratio is $100 \%$, all of the run data are attributed to the blanks. If the maximum blank ratio is zero, no blank had detected values. Generally, data with maximum blank ratios of less than $20 \%$ are considered to be of higher quality. In addition, since determinations of external audit samples for polychlorinated dibenzo-p-dioxins-polychlorinated dibenzofurans (PCDD-PCDF) were not performed, further evaluation of the data quality based on the PCDD-PCDF recoveries was not possible. EER reported that confidence in emission levels of these detected compounds is fairly low because the maximum levels in the blanks are $100 \%$ or greater than the levels in the samples (19).

PCDD-PCDF data obtained at the stack indicate that PCDD-PCDF emissions were very low, ranging from $6.58 \times 10^{-7}$ to $1.07 \times 10^{-5} \mathrm{lb} / 10^{12} \mathrm{Btu}$, with most congeners not detected or near the background (blank) levels. PCDD-PCDF congeners with blank-corrected values above the detection limit reported by EER include 2378-TCDF (tetrachloro dibenzofurans), total PeCDF, total HpCDF, 1234678HpCDD, 1234678-HpCDF, OCDF, and OCDD. Fifty percent of 2378-TCDF average values in the report were attributed to undetected data, and other detected compounds had high blank levels. Therefore, despite surrogate spike recoveries that met EER QA objectives for all compounds, the PCDD-PCDF results at the stack are considered by EER to be questionable (19).

\subsubsection{Effect of Sootblowing on Trace Element Emissions}

The effect of sootblowing on trace element concentrations in stack particulate and corresponding emission factors was evaluated at two of the nine process configurations. During sootblowing, one station (cyclone furnace/ESP) showed a slight enrichment of trace elements, greater total particulate, and higher emission 
factors while the other station (dry-bottom, opposed wall-fired furnace/ESP) showed a slight depletion of trace elements, less total particulate, and lower emission factors. However, the differences between sootblowing and nonsootblowing trace elements concentrations for both sites were not statistically significant.

Four ash streams: bottom ash, economizer ash, and two fields from the ESP hopper ash were sampled at Baldwin Station. While some trace element concentration differences were noted between sootblowing and nonsootblowing periods, none were statistically significant at the $95 \% \mathrm{Cl}$. Although emission factors increased during sootblowing as a result of the increased particulate flow rates, all emission factors remained within the same order of magnitude as those during nonsootblowing periods. During sootblowing, bottom ash samples showed slightly lower concentrations in $\mathrm{Sb}$ and $\mathrm{Be}$, while economizer ash showed slightly higher concentrations in $\mathrm{Sb}, \mathrm{As}, \mathrm{B}, \mathrm{Cd}, \mathrm{Cr}, \mathrm{Cu}$, and $\mathrm{Pb}$. Total particulate increased during the sootblowing by $17 \%$ in the economizer ash and by $3 \%$ to $4 \%$ in the ESP hopper ash. Because of the increased particulate, emission factors for most trace elements were greater during sootblowing periods for all elements except $\mathrm{As}$ and $\mathrm{Ba}$.

During sootblowing at the Cardinal Station, ash samples showed slightly lower concentrations in $\mathrm{Sb}, \mathrm{As}, \mathrm{Be}, \mathrm{B}, \mathrm{Cd}, \mathrm{Cr}, \mathrm{Co}, \mathrm{Cu}, \mathrm{Pb}, \mathrm{Mo}, \mathrm{Ni}, \mathrm{Se}$, and $\mathrm{V}$. $\mathrm{Ba}, \mathrm{Mn}$, and Ag were slightly higher. Total particulate levels were greater during nonsootblowing than during sootblowing, which in turn were greater than levels measured during organic sampling. Data collected during sootblowing operation had less variability than data collected during nonsootblowing operation. All emission factors for nonsootblowing periods, except $\mathrm{Hg}$ and $\mathrm{Mn}$, were higher than those for sootblowing periods. This is consistent with the fact that higher particulate levels were measured during nonsootblowing operation than during sootblowing periods.

From the limited data collected at both sites, no statistically significant trends were observed with respect to the effect of sootblowing on particulate trace element concentrations and emission factors or total quantity of particulate. However, because the sootblowing tests at Baldwin Station utilized duct traverses during sampling rather than single-point measurements utilized at the Cardinal Station, the 
Baldwin Station data are believed to be more representative of actual sootblowing effects.

\subsubsection{HAPs on Particle Surfaces}

The CAAA of 1990 mandate that emissions of HAPs from coal-fired power plants be evaluated for potential health risks. The condensed metal species found predominantly on the surface of fly ash particles are readily accessible to biological and ecological systems and thus pose a potentially greater health risk than do those species trapped in the aluminosilicate fly ash matrix. Surface enrichment of HAPs is thought to occur when metal species in the gas phase condense onto the surfaces of the fine particles, which are more difficult to remove by control devices.

In order to address this public health issue, DOE funded a separate test program, conducted by Radian, to compare bulk and surface composition of particulate samples (14). Flue gas particulate samples were collected at Plant Yates, including gas from the outlet of the JBR and the ESP inlet and outlet.

Extractable metal concentrations were determined for bulk particulate samples. The elements studied include $\mathrm{Sb}, \mathrm{As}, \mathrm{Ba}, \mathrm{Be}, \mathrm{Cd}, \mathrm{Cr}, \mathrm{Cu}, \mathrm{Co}, \mathrm{Pb}, \mathrm{Mn}, \mathrm{Mo}, \mathrm{Ni}, \mathrm{Se}$, and $\mathrm{V}$. Hg was initially included in the test program, but the results were invalidated by poor matrix spike and blank spike recoveries. The premise of this study is that extractable concentrations of these elements indicate their leachability and thus their potential availability to biological and ecological systems. Acid leaching and digestions of the particulate samples were followed by ICP-MS analysis. Leachability is influenced by a number of factors, including solubility, particle surface area, surface concentration, and other matrix effects.

Three separate sample preparation techniques were used, as illustrated in Figure 4-26. A nitric acid digestion was used to represent the highest degree of surface availability for elements not bound in the aluminosilicate fly ash matrix. A hydrochloric acid digestion was used to simulate human ingestion. Finally, an acetic acid digestion was used to simulate groundwater leaching mechanisms. In addition, 
the total composition of each sample was obtained from the trace element analysis of the particulate at the ESP inlet and outlet and from analysis of the stack gas EPA Method 29 filter samples.

In general, increasing extractability was observed along the flue gas path, as shown in Figures 4-27 through 4-40. It is reasonable to expect higher extractable concentrations at the ESP outlet compared to the inlet, based solely on the reduction in mean particle diameter across the ESP. The increased surface area associated with an equivalent sample mass exposes more material to the leaching solutions.

Table 4-16 shows the metals arranged in order of leachability, based on results for the ESP inlet and outlet samples.

Elements exhibiting the highest degree of extractability, such as Mo, Cd, and $\mathrm{Sb}$, are likely to be surface-oriented, unbound in the particle matrix, or in a form readily dissolved by the leaching agent. The spike recovery results shown in Table 4-16 indicate that the data for As, Se, Mn; and V cannot be considered reliable.

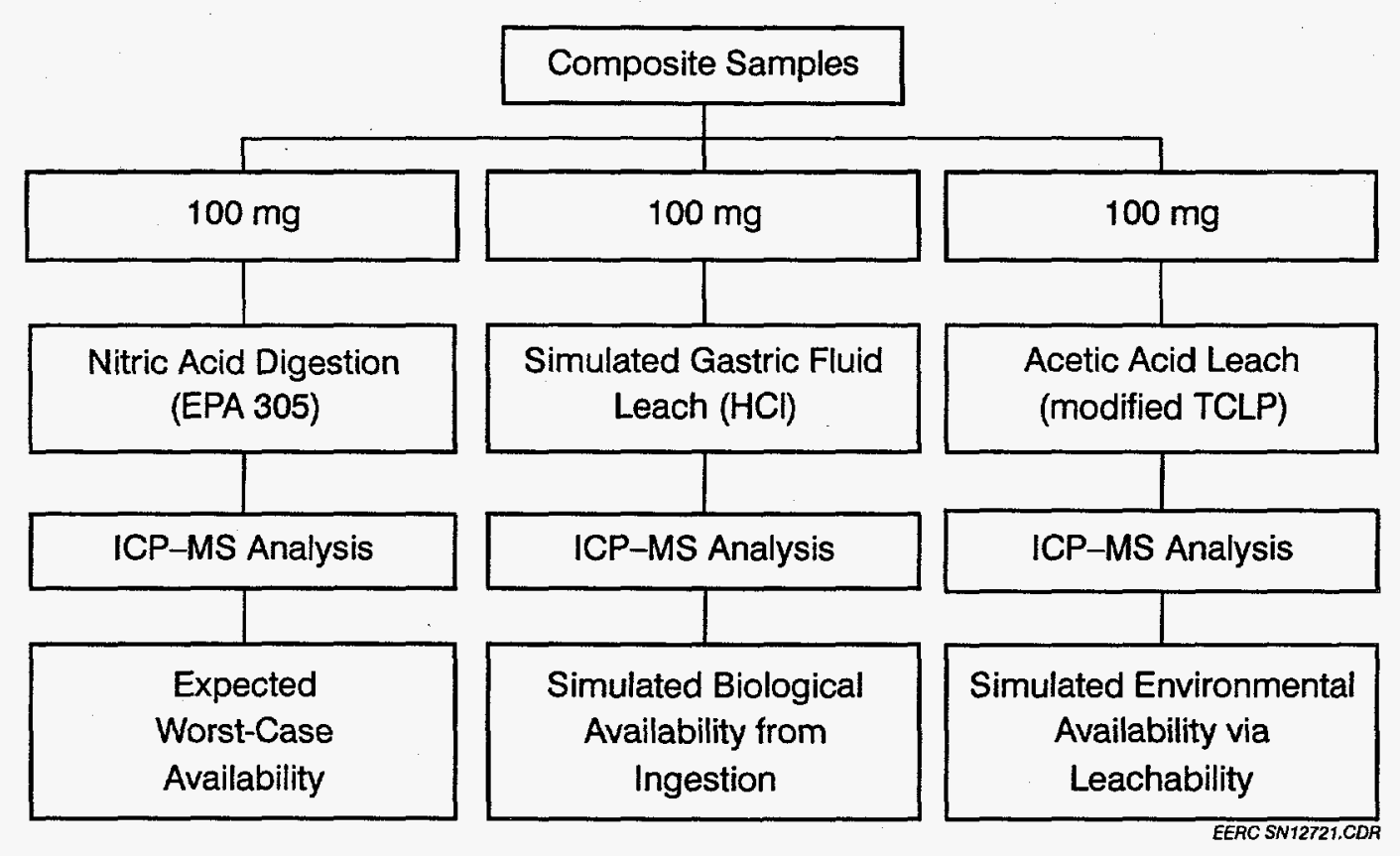

Figure 4-26. Flue Gas Particulate Sample Preparation and Analysis Plan for Extractable Metals (13). 


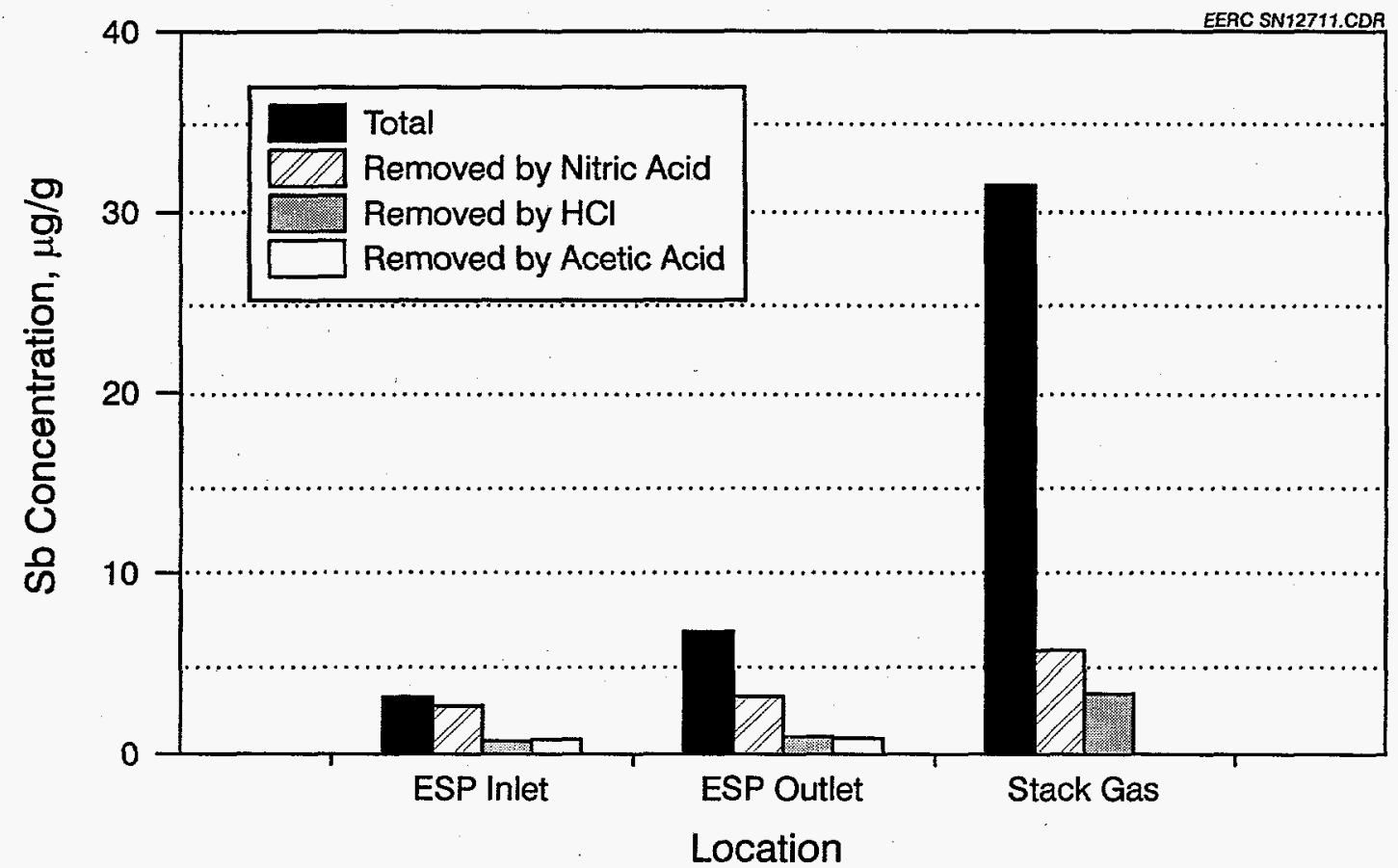

Figure 4-27. Sb Content of Flue Gas Particulate as Sampled at Plant Yates.

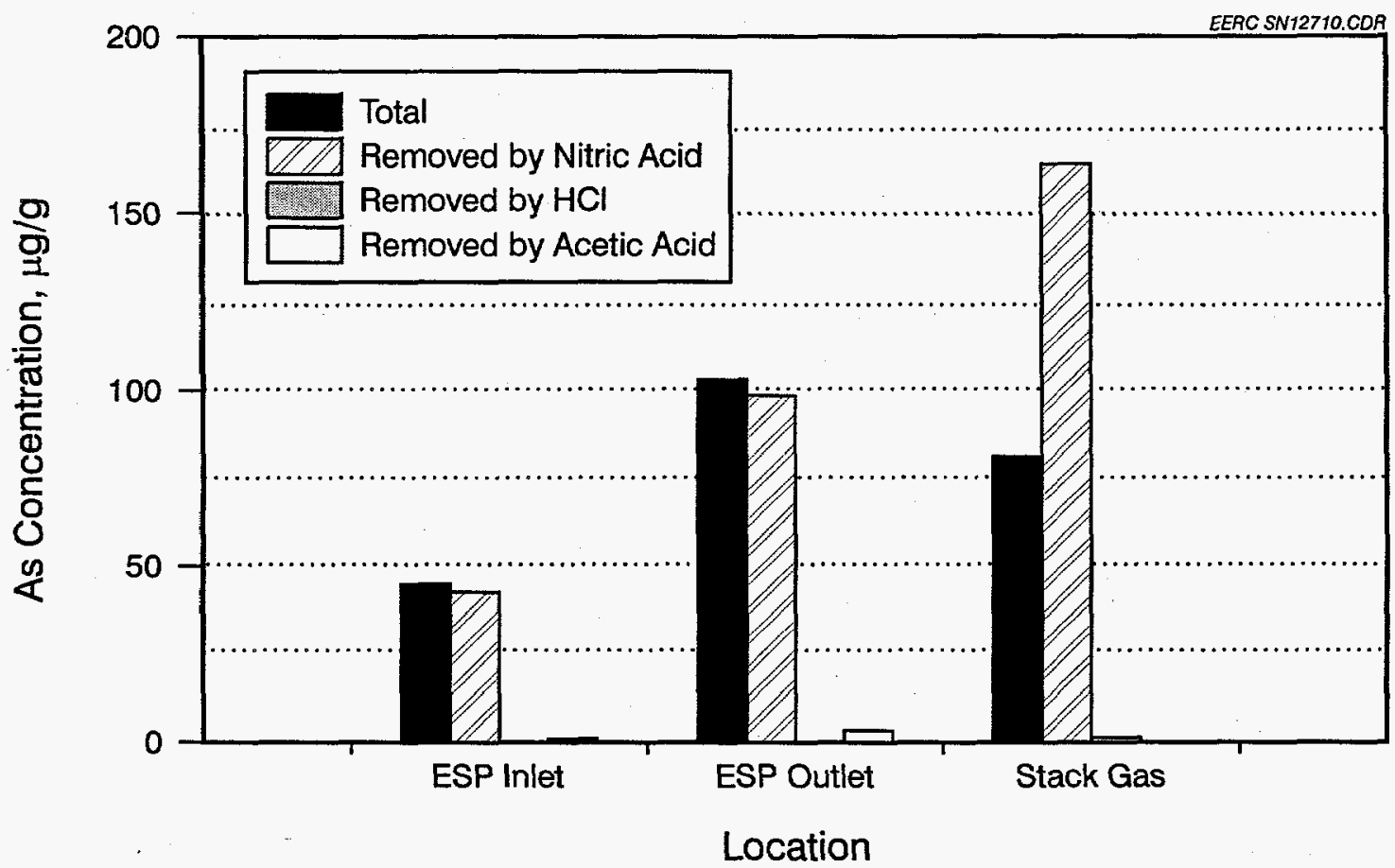

Figure 4-28. As Content of Flue Gas Particulate as Sampled at Plant Yates. 


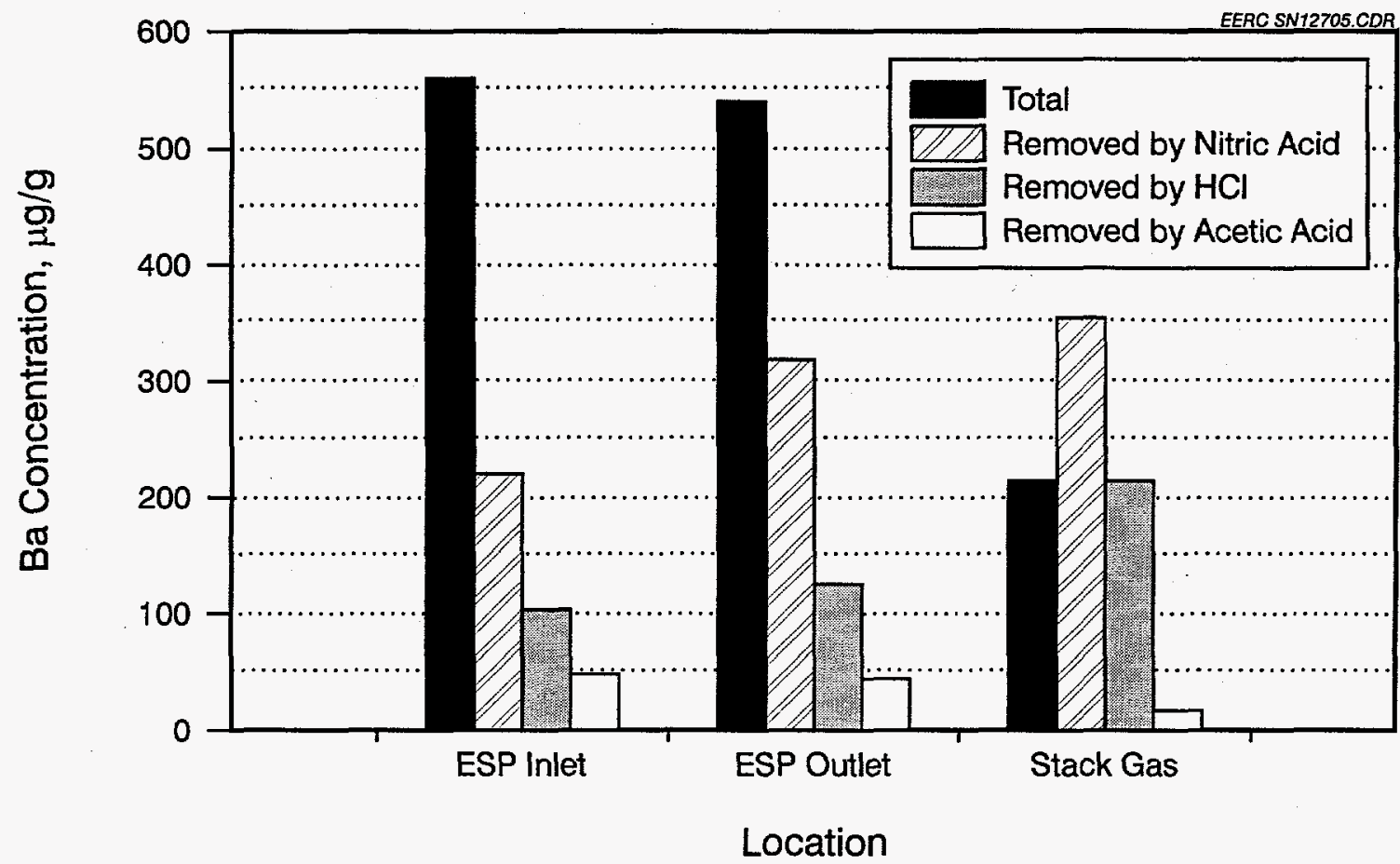

Figure 4-29. Ba Content of Flue Gas Particulate as Sampled at Plant Yates.

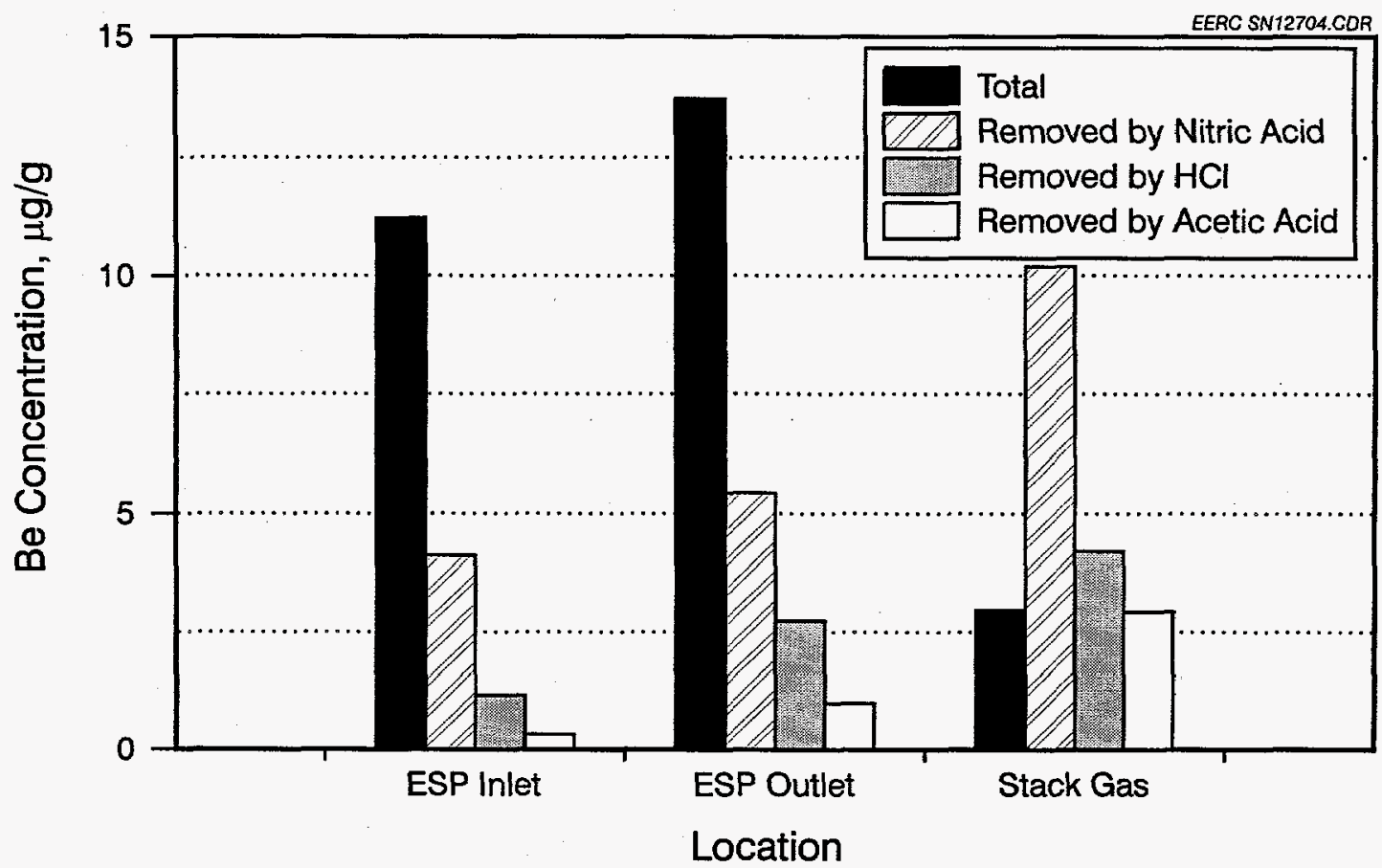

Figure 4-30. Be Content of Flue Gas Particulate as Sampled at Plant Yates. 


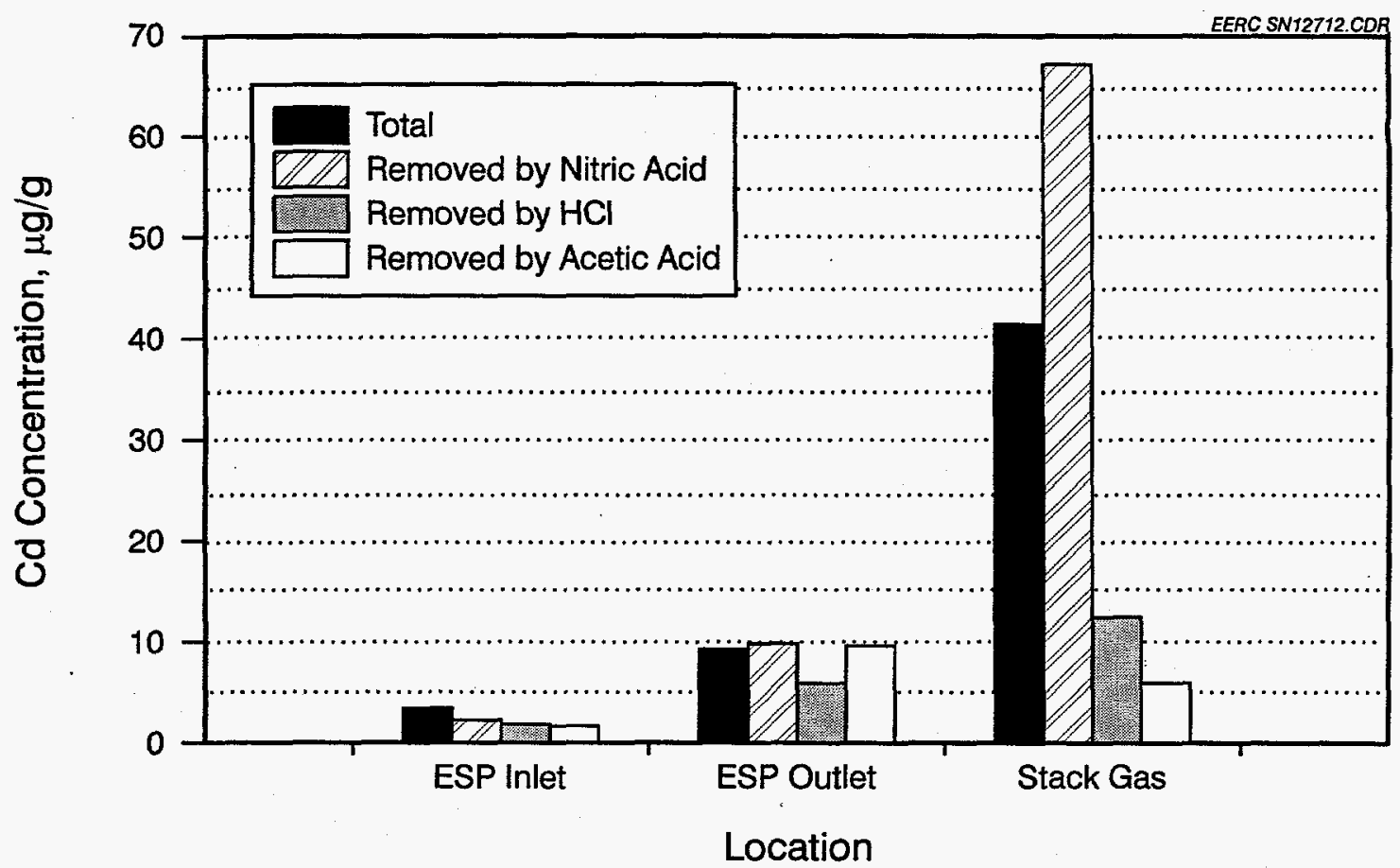

Figure 4-31. Cd Content of Flue Gas Particulate as Sampled at Plant Yates.

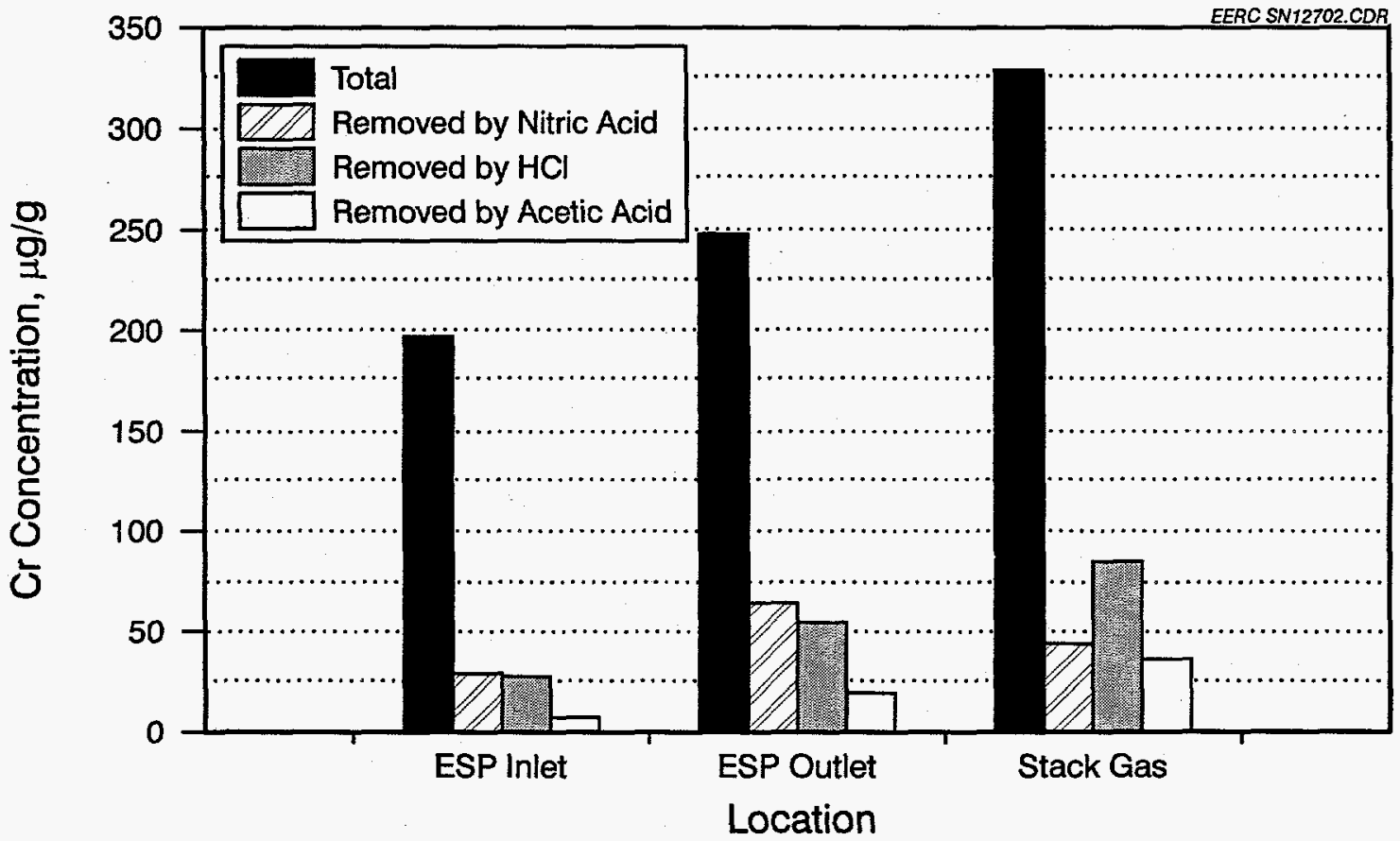

Figure 4-32. Cr Content of Flue Gas Particulate as Sampled at Plant Yates. 


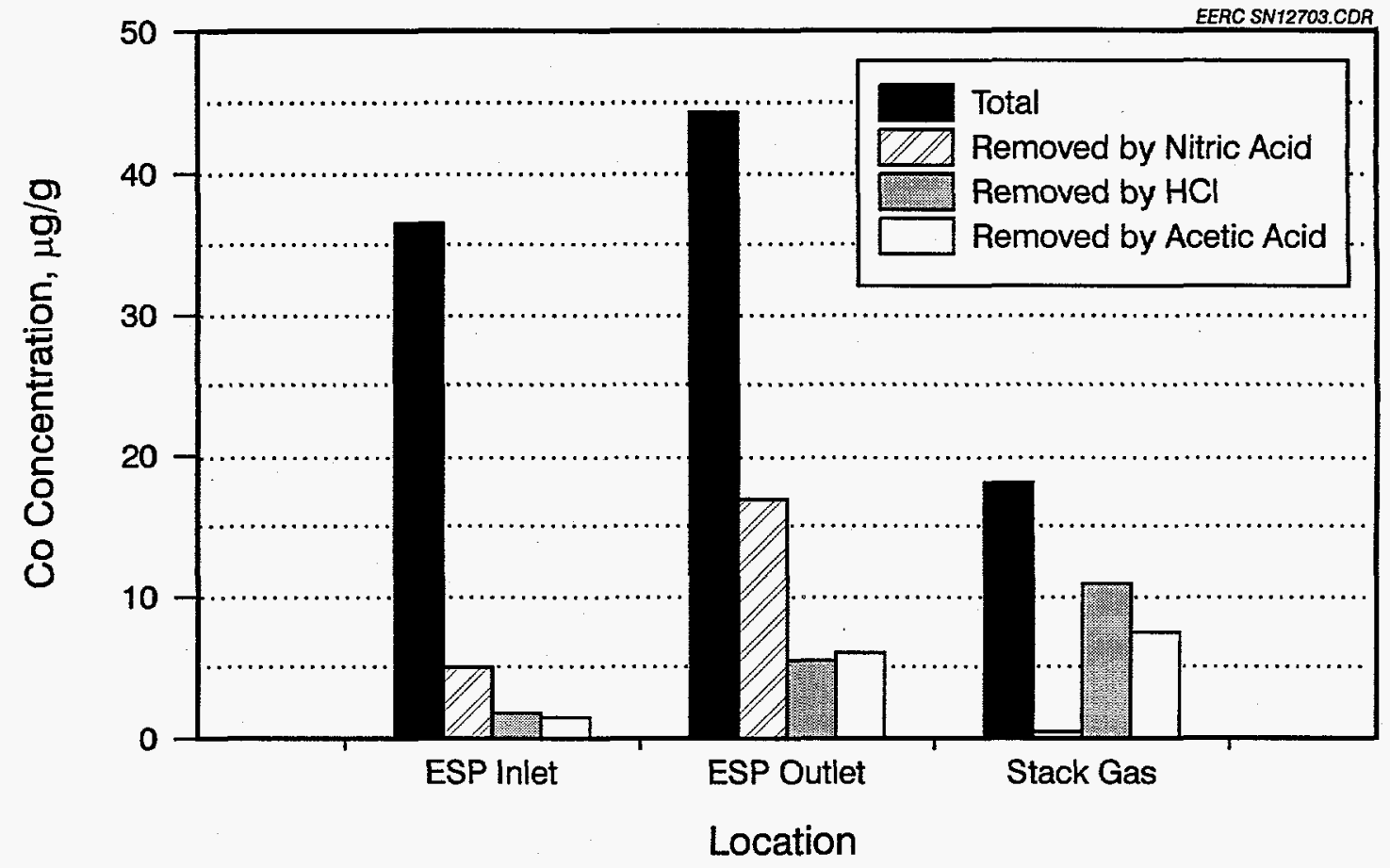

Figure 4-33. Co Content of Flue Gas Particulate as Sampled at Plant Yates.

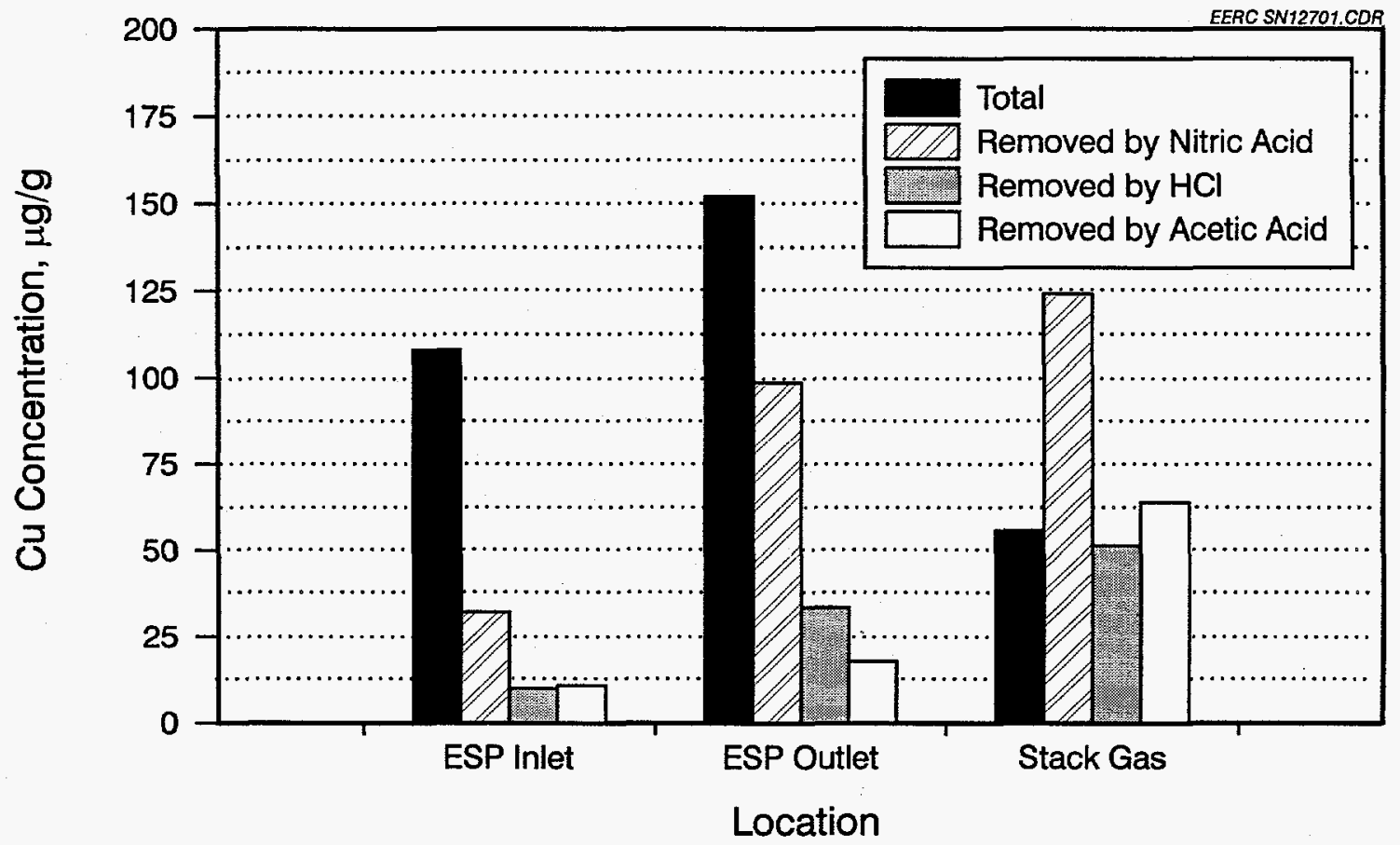

Figure 4-34. Cu Content of Flue Gas Particulate as Sampled at Plant Yates. 


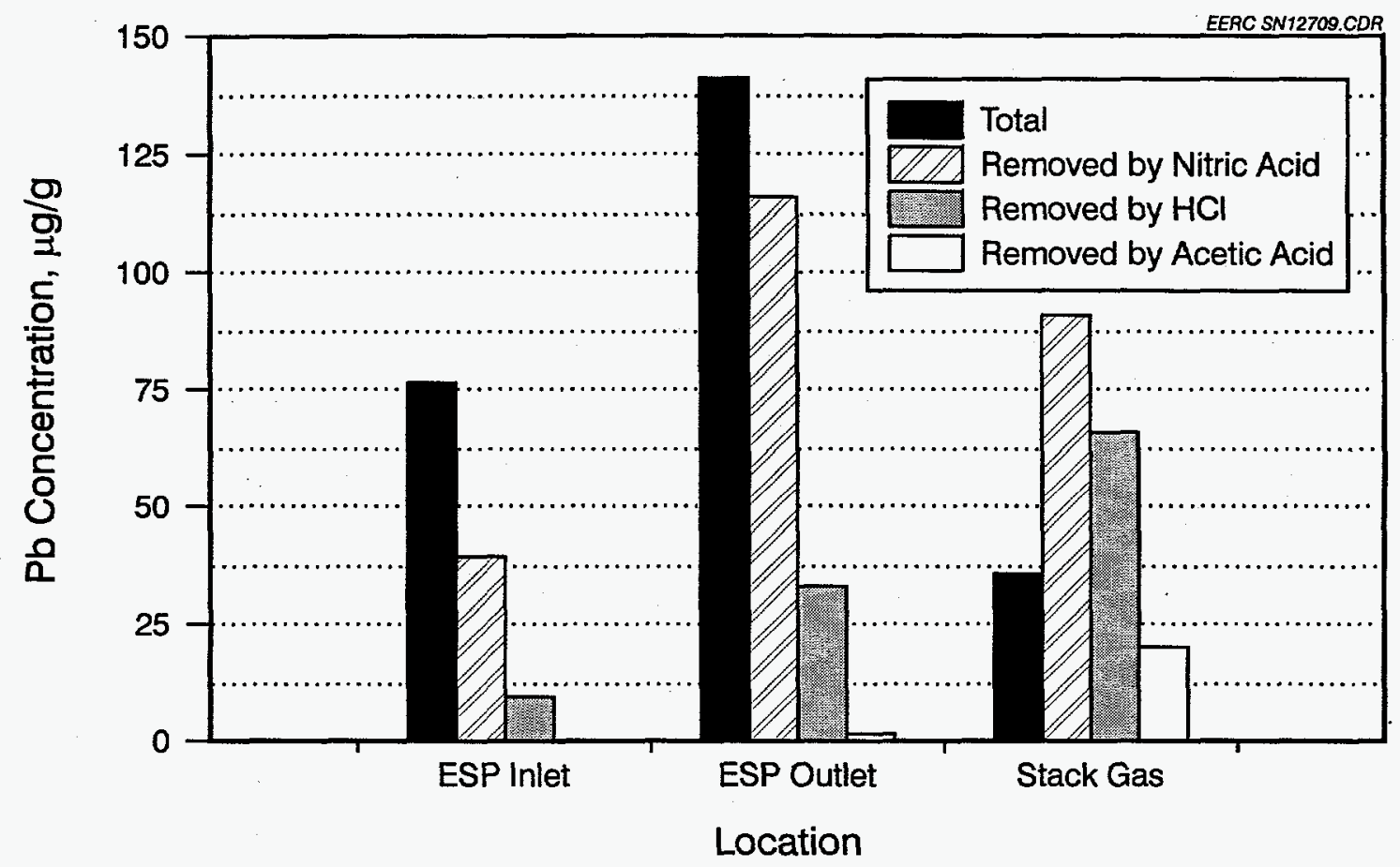

Figure 4-35. $\mathrm{Pb}$ Content of Flue Gas Particulate as Sampled at Plant Yates.

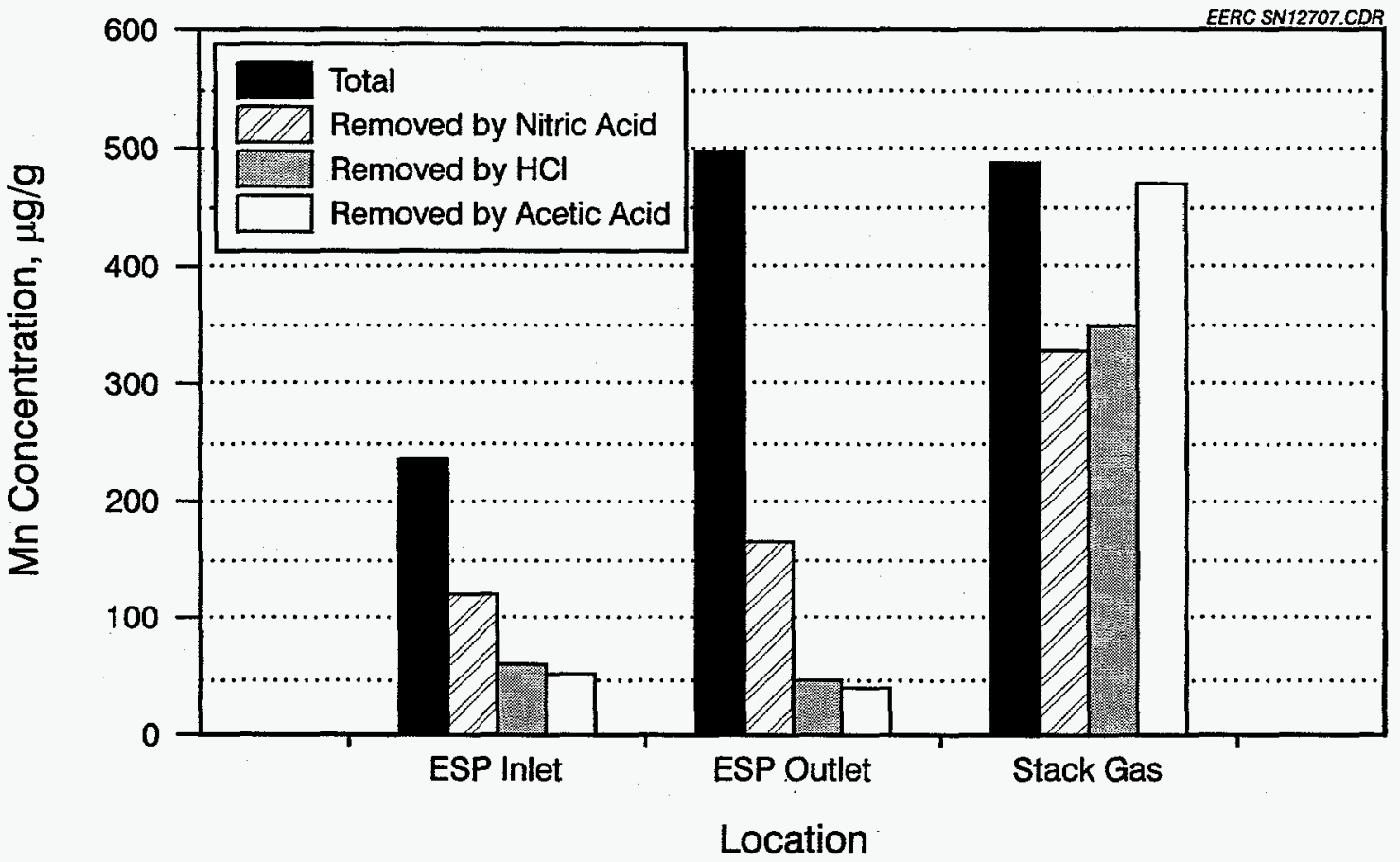

Figure 4-36. Mn Content of Flue Gas Particulate as Sampled at Plant Yates. 


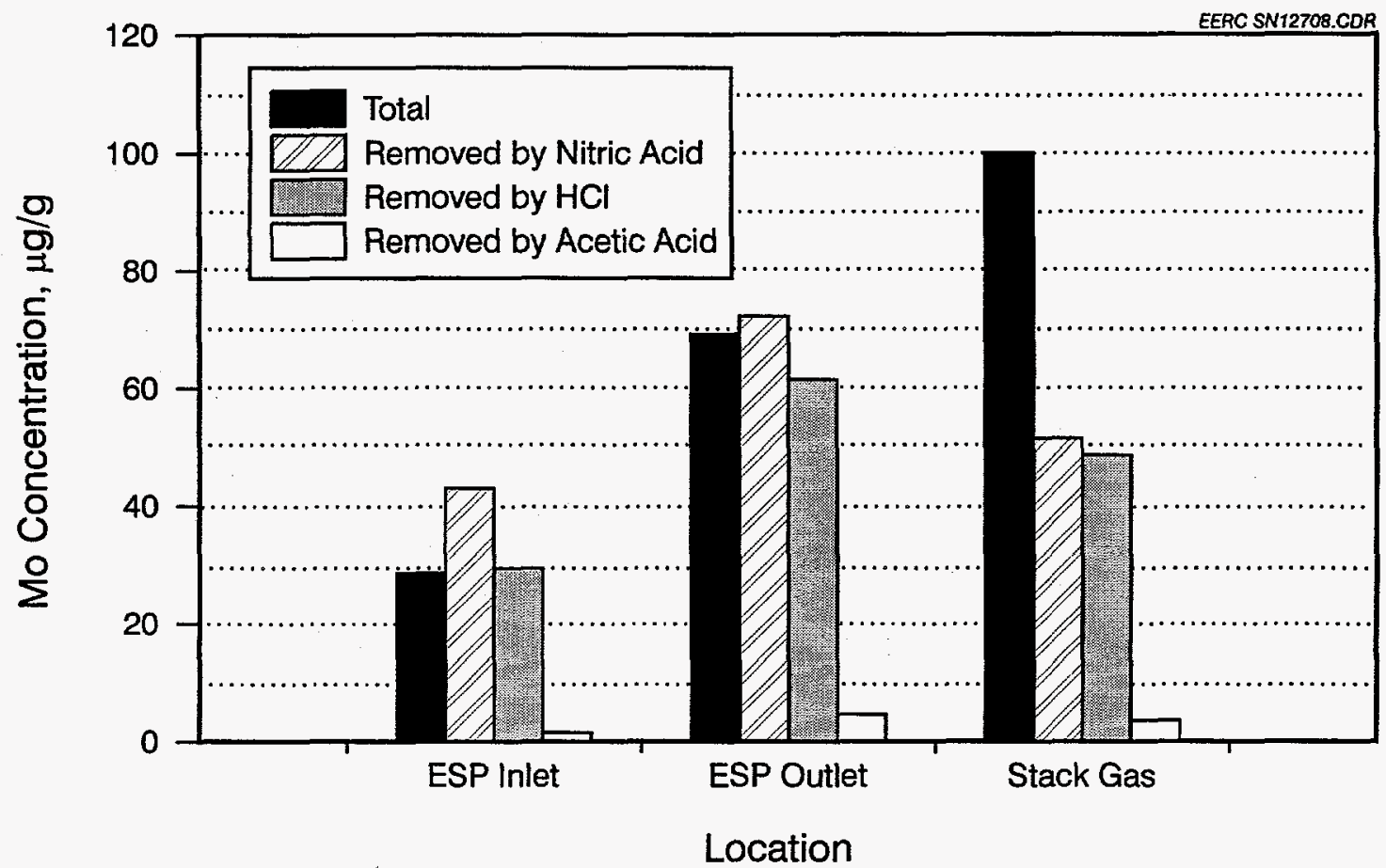

Figure 4-37. Mo Content of Flue Gas Particulate as Sampled at Plant Yates.

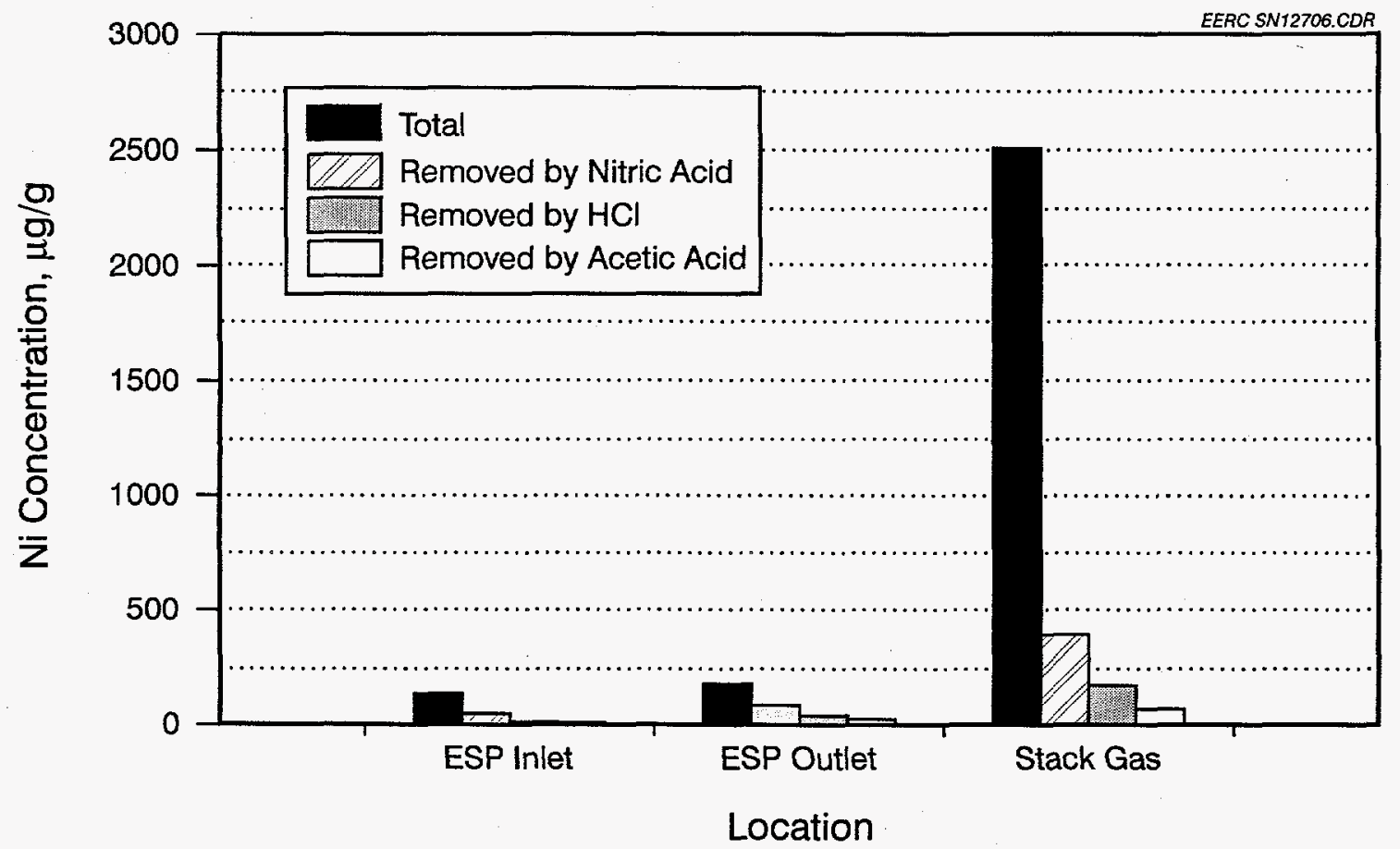

Figure 4-38. Ni Content of Flue Gas Particulate as Sampled at Plant Yates. 


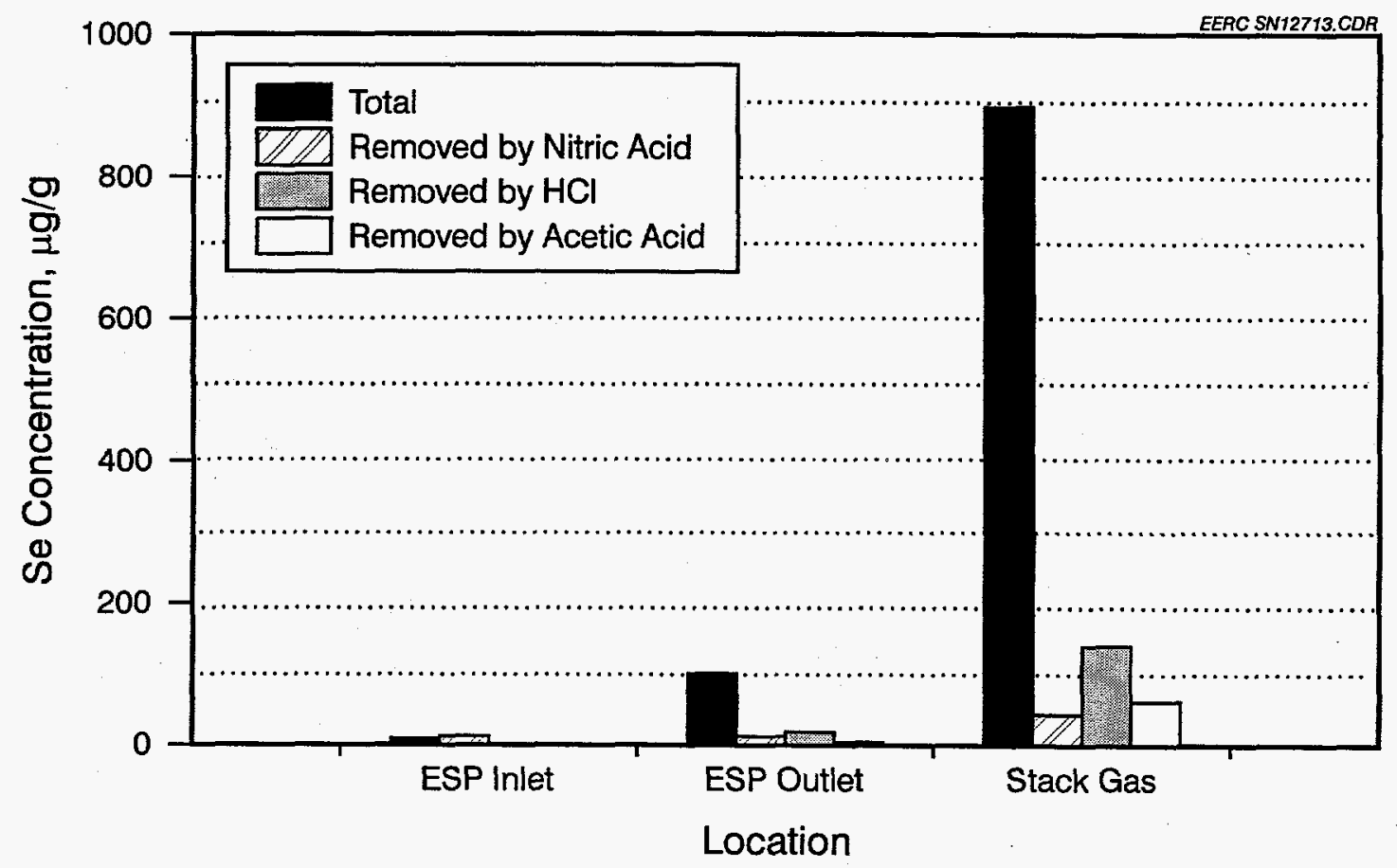

Figure 4-39. Se Content of Flue Gas Particulate as Sampled at Plant Yates.

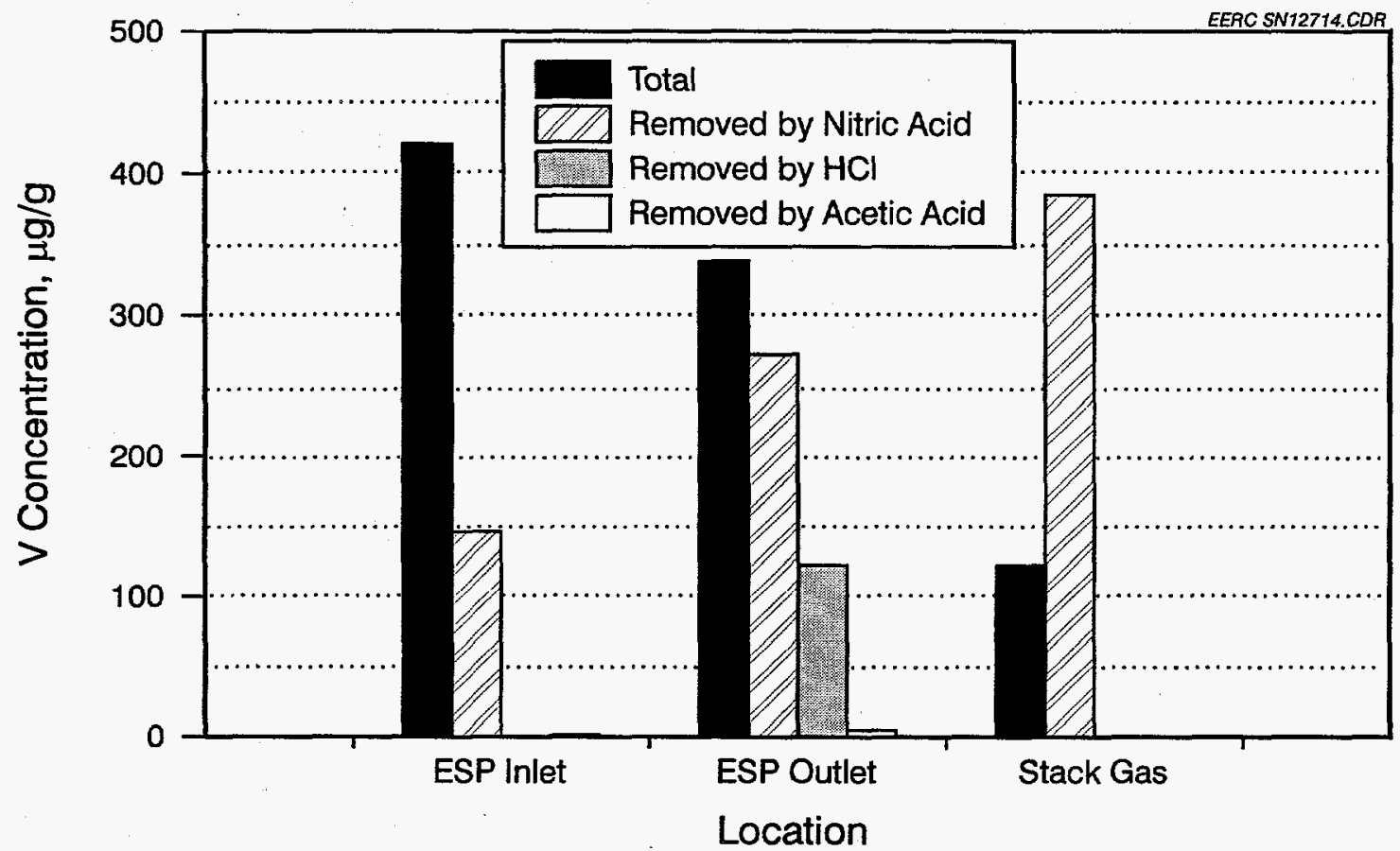

Figure 4-40. V Content of Flue Gas Particulate as Sampled at Plant Yates. 
Table 4-16. Average Extractability of Elements in Fly Ash

\begin{tabular}{|c|c|c|c|c|c|}
\hline \multirow[b]{2}{*}{ Element } & \multicolumn{3}{|c|}{ Average \% Extractable } & \multicolumn{2}{|c|}{ Spike Recovery, \% } \\
\hline & ESP Inlet & ESP Outlet & Stack Gas & Average & Range \\
\hline $\mathrm{Sb}$ & 44 & 25 & 9.7 & $N^{a}$ & NA \\
\hline As & 33 & 33 & $68^{b}$ & 81 & $0-123^{b}$ \\
\hline $\mathrm{Ba}$ & 22 & 30 & $91^{\mathrm{b}}$ & 90 & $85-94$ \\
\hline $\mathrm{Be}$ & 17 & 22 & $197^{b}$ & 93 & $79-108$ \\
\hline $\mathrm{Cd}$ & 55 & $91^{b}$ & $69^{b}$ & 96 & $88-107$ \\
\hline $\mathrm{Cr}$ & 11 & 19 & 17 & 98 & $92-100$ \\
\hline Co & 7.6 & 21 & 35 & 98 & $92-100$ \\
\hline $\mathrm{Cu}$ & 16 & 33 & $143^{b}$ & 99 & $92-105$ \\
\hline $\mathrm{Pb}$ & 21 & 36 & $165^{\mathrm{b}}$ & 88 & $83-97$ \\
\hline $\mathrm{Mn}$ & 33 & 17 & 78 & 89 & $71-108^{\circ}$ \\
\hline Mo & $86^{b}$ & $67^{b}$ & 35 & NA & NA \\
\hline $\mathrm{Ni}$ & 16 & 27 & 8.3 & 95 & $81-103$ \\
\hline $\mathrm{Se}$ & $48^{b}$ & 11 & 9.1 & 117 & $84-138^{\circ}$ \\
\hline $\mathrm{V}$ & 12 & 30 & $105^{b}$ & 71 & $0-109^{\circ}$ \\
\hline
\end{tabular}

In addition, the leaching results contain some data that exceed $100 \%$ of the bulk compositions for a certain element, particularly for the nitric acid extractions of the stack gas samples, indicating a possible sample preparation problem.

Several elements ( $\mathrm{As}, \mathrm{Ba}, \mathrm{Be}, \mathrm{Co}, \mathrm{Cu}, \mathrm{Pb}$, and $\mathrm{V})$ are found in the stack gas samples at concentrations lower than in the ESP outlet (FGD inlet) samples. This reduction in elemental concentrations across the JBR, despite the reduction in mean particle diameter, suggests that these elements may be leached from fly ash by the FGD slurry. Other results obtained during the Plant Yates study indicated that the JBR slurry is enriched in aqueous $\mathrm{Cd}, \mathrm{Pb}, \mathrm{Mn}, \mathrm{Cu}, \mathrm{Se}, \mathrm{Co}, \mathrm{As}, \mathrm{Ni}, \mathrm{V}, \mathrm{Be}$, and $\mathrm{Cr}$ relative to the concentration of soluble silica in the recycled slurry after six cycles. Molybdenum and As are also enriched in the JBR slurry's solid phase. This concentration mechanism is not well understood but appears to impact particle surface characterization for particulate matter downstream of wet-scrubbing systems. The extractability data presented for Plant Yates cannot be interpreted without a better understanding of the enrichment of trace elements in the slurry and carryover of fine particles to the stack. 


\subsection{CONCLUSIONS AND RECOMMENDATIONS}

Specific conclusions and recommendations are summarized below for individual topics relating to the round-robin coal analyses, emission factors for inorganic trace elements, organic compounds, radionuclides, acid gases and halogens, and several special topics.

\subsection{Round-Robin Coal Analyses}

Results were obtained for the 17 trace elements ( $\mathrm{Sb}, \mathrm{As}, \mathrm{Ba}, \mathrm{Be}, \mathrm{B}, \mathrm{Cd}, \mathrm{Cr}, \mathrm{Co}$, $\mathrm{Cu}, \mathrm{Pb}, \mathrm{Mn}, \mathrm{Hg}, \mathrm{Mo}, \mathrm{Ni}, \mathrm{Se}, \mathrm{V}$, and $\mathrm{F}$ ) included in the round-robin study. The percentage of accurate trace element analyses of a NIST standard la Pittsburgh seam coal) ranged from $38 \%$ to $75 \%$. Accurate values were considered to be those within $10 \%$ of the NIST-certified value. The elements yielding the most problematic trace element data were $\mathrm{Sb}, \mathrm{As}, \mathrm{Cd}, \mathrm{Mo}$, and $\mathrm{Se}$. No laboratory was able to report trace element content accurately more than $75 \%$ of the time.

Interlaboratory reproducibility was evaluated using PRSD. The average PRSD for all coals and all contractors was $28 \%$. Average PRSD values for individual trace elements ranged from $11 \%$ for $V$ to $61 \%$ for Mo. The range of PRSDs was large: $\mathrm{Ba}$, $\mathrm{Cd}, \mathrm{Cu}, \mathrm{Hg}, \mathrm{Mo}, \mathrm{Ni}, \mathrm{Pb}$, and $\mathrm{Sb}$ all have $\mathrm{PRSD}$ ranges of over $30 \%$. Average PRSD was found to be correlated to coal heating value, indicating that as coal rank decreases, the analytical variability for trace elements increases.

Intralaboratory repeatability was calculated as the average percent difference in a single laboratory's results on eight duplicate samples. The average percent difference for trace elements was $15 \%$, ranging from a low of $7.8 \%$ for $\mathrm{Cr}$ to $33 \%$ for $\mathrm{Cd}$. Elements with low interlaboratory reproducibility also tended to have low intralaboratory repeatability.

Comparison of the round-robin results with the plant data showed major differences. In many cases, the plant results differed from the round-robin results by $25 \%$ or more for major element and proximate-ultimate values as well as trace element 
results. At times, these differences exceeded $100 \%$. Coal trace element content was observed to vary within about 1 order of magnitude for each element. These results are problematic, suggesting that the feed coal data used in mass balance and penetration calculations are a major source of uncertainty.

The results of the round-robin study demonstrate that significant problems exist in trace element analyses of coals. Laboratories to be used for future analytical work should be screened using ASTM Standard Method D4182, Evaluation of Laboratories Using ASTM Procedures in the Sampling and Analysis of Coal and Coke, and ASTM Standard Method D4621, Accountability and Quality Control in the Coal Analysis Laboratory immediately prior to future field efforts. Only laboratories meeting these ASTM standards should be allowed to perform analyses in future studies. Laboratories could be evaluated by having them analyze certified standard reference materials within specified precision limits for all sample types to be characterized.

The presence of numerous nondect values in the data sets further contributes to the difficulty of predicting power plant emissions. The practice of using half the detection limit to represent a nondect value in emission factor calculations should be avoided. Future field testing and analysis programs should specify the minimum detection limits for each element.

\subsection{ORganic Emission Factors}

In general, the emission rates were low for four types of organic compound classes sampled (aldehydes and ketones, VOCs, SVOCs, and PAHs, and chlorinated dioxins and furans) from the DOE Phase I field sites.

Despite the QA/QC problems with some of the organic emission data, those obtained in the Phase I investigation have provided an overall representation that emissions of organic pollutants attributable to coal-fired power plants are very low. The low concentrations of most of the organic compounds found in the stacks of the nine process configurations were typically within an order of magnitude of the concentrations previously reported by the EPA for ambient air. 


\subsection{Trace Element Emission Factors}

The variability of the DOE Phase I data resulting from fuel variability and sampling/analytical precision demonstrate the difficulty involved in quantifying trace element emissions from coal-fired systems. The data show that the emission factor range for a given element was from 1 order of magnitude for $\mathrm{Mn} 12.6$ to $\left.30 \mathrm{lb} / 10^{12} \mathrm{Btu}\right)$ and $\mathrm{Hg}\left(1.9\right.$ to $\left.22 \mathrm{lb} / 10^{12} \mathrm{Btu}\right)$ to nearly 4 orders of magnitude for Se (ND $<0.038$ to $193 \mathrm{lb} / 10^{12} \mathrm{Btu}$ ). The trace element listed as a HAP having the highest median emission factor was Se $\left(26.5 \mathrm{lb} / 10^{12} \mathrm{Btu}\right)$, followed closely by $\mathrm{Mn}$ $\left(11 \mathrm{lb} / 10^{12} \mathrm{Btu}\right) . \mathrm{Sb}$ and $\mathrm{Be}$ were observed to have the lowest median emission factors, ND $<0.36$ and ND $<0.13 \mathrm{lb} / 10^{12} \mathrm{Btu}$, respectively.

Emission factors for total $\mathrm{Hg}$ ranged from 1.9 to $22 \mathrm{lb} / 10^{12}$ Btu based on mean stack concentrations of 2.6 to $30 \mu \mathrm{g} / \mathrm{Nm}^{3}$. Typical mean $\mathrm{Hg}$ values of about $0.1 \mu \mathrm{g} / \mathrm{g}$ were reported for six of the eight fuels. Calculated percent penetration values for total $\mathrm{Hg}$ ranged from about $25 \%$ to nearly $120 \%$. From the DOE Phase I data, the potential to control $\mathrm{Hg}$ emissions from coal-fired utility boilers using existing emission control technologies is unclear. It is also important to remember that the control of volatile or vapor-phase HAPs is not likely to exceed control levels observed for vapor-phase priority pollutants such as $\mathrm{SO}_{2}$. Therefore, if $\mathrm{Hg}$ regulations are promulgated for coalfired utility systems, existing control technologies will require augmentation, and alternative control technology options will require development.

Evaluating emission control technology performance based on total $\mathrm{Hg}$ concentration alone is not appropriate, since $\mathrm{Hg}$ speciation may affect the degree of control observed. This effect currently cannot be quantified adequately since methods to speciate $\mathrm{Hg}$ are still unproven. Future $\mathrm{Hg}$ sampling efforts must emphasize accurate $\mathrm{Hg}$ speciation in order to evaluate the performance of emission control technologies properly.

Emission control for the 13 nonvolatile trace elements was $>90 \%$ for all nine process configurations based on plant coal data, representing a wide range of firing configurations, fuel types, and emission control technologies. Particulate control alone 
(ESP or FF) limited trace element penetration to $5 \%$ or less (>95\% control) for ten (Sb, $\mathrm{Ba}, \mathrm{Be}, \mathrm{Cr}, \mathrm{Co}, \mathrm{Cu}, \mathrm{Pb}, \mathrm{Mn}, \mathrm{Ni}$, and $\mathrm{V}$ ) of the 13 trace elements. A combination of particulate control and SDA or wet FGD demonstrated $>99 \%$ trace element control for eight ( $\mathrm{Sb}, \mathrm{As}, \mathrm{Ba}, \mathrm{Co}, \mathrm{Cu}, \mathrm{Pb}, \mathrm{Mn}$, and $\mathrm{V}$ ) of the 13 trace elements. The exceptions noted appear to be the result of high reported nondetect concentrations, failed blanks and/or spike recoveries, and significant data variability. Therefore, the DOE Phase I data generally indicate that the emission of these 13 trace elements is effectively controlled (>98\%) by highly efficient particulate control technology or technology combinations (ESP-scrubber or SDA-FF) currently being used by the U.S. utility industry. However, the control of trace element emissions will never exceed the level of overall particulate control observed.

A comparison of DOE Phase I stack concentration data with ambient air concentration data collected since 1980 for 11 trace elements shows that for nine (Sb, As, $\mathrm{Be}, \mathrm{Cd}, \mathrm{Co}, \mathrm{Cr}, \mathrm{Hg}, \mathrm{Mn}$, and $\mathrm{Ni}$ ) of the 11 trace elements, the median stack concentrations are 2 to 3 orders of magnitude greater than the range of ambient air concentrations. For $\mathrm{Se}$ and $\mathrm{Pb}$, the differential was roughly 4 and 1 order of magnitude, respectively. These data imply, with the exception of $\mathrm{Pb}$, that coal-fired power plants are possible contributors to ambient air concentrations for these trace elements. However, the level or degree of contribution can be determined only as a function of extensive dilution and dispersion modeling.

The CAAA of 1990 will reduce and ultimately cap $\mathrm{SO}_{2}$ emissions from coal-fired electrical generating facilities by the year 2000. As a result, the installation of additional FGD capacity to achieve the $1.2 \mathrm{lb} / \mathrm{MMBtu} \mathrm{SO}_{2}$ limit and meet the emission cap will also significantly reduce the emissions of trace elements.

Based on fuel feed rates and analyses reported for the nine process configurations and an overall average capacity factor of 0.7 , the total annual emission rates at each plant for individual trace element ( $\mathrm{Sb}, \mathrm{As}, \mathrm{Be}, \mathrm{Cd}, \mathrm{Cr}, \mathrm{Co}, \mathrm{Pb}, \mathrm{Mn}, \mathrm{Hg}, \mathrm{Ni}$, and $\mathrm{Se}$ ) $\mathrm{HAPs}$ were all estimated to be $<3$ tons/yr, and most were substantially $<0.5$ tons/yr. The estimated combined trace element HAP annual emission rates for each of the nine process configurations ranged from 0.06 to 5.65 tons/yr. 


\subsection{RADIONUCLIDE EMISSION FACTORS}

Most measured activities were nondetects. Average emission factors for detected values on an activity basis were in the range of $1.4 \times 10^{8} \mathrm{pCi} / 10^{12} \mathrm{Btu}$ for Ra-226 to $7.2 \times 10^{10} \mathrm{pCi} / 10^{12} \mathrm{Btu}$ for $\mathrm{Pb}-210$. On a mass basis, emission factors ranged from $3.9 \times 10^{-10} \mathrm{lb} / 10^{12}$ Btu for Po-210 to $312 \mathrm{lb} / 10^{12}$ Btu for U-238. The radionuclide data set suggests that radionuclide emissions are very low for the Phase I coal-fired power plants.

\subsection{AcID Gases-Halogen Emission Factors}

Acid gas speciation was performed at two ESP sites and one FF site. Chlorine gas concentrations were significant for the FF site but not at the ESP sites. Fluorine gas concentrations were significant for one ESP site, but were not significant at the second ESP site or the FF site.

Scrubber systems were effective in removing acid gases from flue gas. ESPS were highly effective in removing particulate $\mathrm{Cl}^{-}, \mathrm{F}^{-}$and, to a lesser extent, $\mathrm{CN}^{-}$, where greatest removal followed greatest particulate size: $\mathrm{Cl}^{-}>\mathrm{F}^{-}>\mathrm{CN}^{-}$. The ESPs did not remove acid gases. One FF system sampled appears to have removed some acid gases, but the lack of a second similar FF site for comparison does not allow for firm conclusions.

\subsection{SPECIAL TOPICS}

\subsubsection{Simulated Plume Dilution Sampling}

Because of the data quality problems, no firm conclusions can be drawn from the plume dilution sampling results other than the demonstrated need for much more development of this sampling approach. For credible quantitative results, the PSDS method must be verified with rigorous $\mathrm{QA} / \mathrm{OC}$, including blank analysis to ensure that blank corrections are not a significant portion of the measured values. Measured concentrations of the trace elements in the stack flue gas for the diluted and undiluted 
samples must be in good agreement, and the variability of the repeat measurements must be smaller than any observed effect of the cooling and dilution.

\subsubsection{Trace Element Enrichment as a Function of Particle Size}

The data cumulatively suggest that significant enrichment with decreasing particle size is most likely to occur for Sb, As, Mo, and Se. Because of this enrichment, removal efficiency for these trace elements is likely to be lower than total particulate removal efficiency, for control devices such as an ESP where collection efficiency decreases with decreasing particle size.

\subsubsection{Particle-Size Distribution of Fly Ash and Stack Emissions}

The inlet MMDs ranged from 13 to $23 \mu \mathrm{m}$ which is typical of fly ash particle size for coal-fired boilers. Measured stack MMD values of 0.55 to $9.0 \mu \mathrm{m}$ represent a much broader range than the inlet MMDs, demonstrating complex and varying sizefractionating mechanisms. Two of the outlet MMD values may have been influenced by submicron acid condensation, which may significantly skew the stack particle-size distribution toward finer sizes. In all of these cases, the stack MMD was smaller than the inlet MMD, but the ratio of inlet-to-stack MMD ranged from 1.4 to 36 , which demonstrates that the particle size of the stack emission is likely to be highly systemspecific.

\subsubsection{Chromium Speciation and Sampling}

Hexavalent chromium field blank concentrations were higher than the stack flue gas concentrations for the two field sites at which measurements were made. Therefore, based on the DOE Phase I data, hexavalent chromium emissions from coalfired boilers do not appear to be significant. The EERC recommends that a thorough set of validation tests be performed on sampling and analytical protocols for chromium speciation. Once validated, sampling and analytical protocols can be applied to fullscale systems to document chromium speciation and to avoid the current EPA practice in which all chromium emissions are considered to be hexavalent. 


\subsubsection{Dioxins-Furans Across ESPs}

In general, PCDD-PCDF emission factors reported by the contractors were typically $<3 \times 10^{-6}$ to $<6 \times 10^{-6} \mathrm{lb} / 10^{12} \mathrm{Btu}$, which indicates that chlorinated dioxin and furan emissions are not significant from coal-fired power plants included in this investigation.

Based on the PCDD-PCDF data from the flue gas collected at the ESP inlet and outlet (stack), EER reported that the apparent removal efficiency of individual PCDD-PCDF congeners ranged from $0 \%$ to $99 \%$ and generally decreased with increasing chlorination level, which suggested some shifting of PCDD-PCDF congeners to higher chlorination levels in the ESP. This observation made by EER may indicate that PCDDs-PCDFs were formed from precursors in the ESP. Because of the poor data quality from PCDD-PCDF samples obtained at the Cardinal Station's ESP inlet and outlet, no conclusive interpretation of the data can be made, and so further investigation is needed to confirm this observation.

\subsubsection{Effect of Sootblowing on Trace Element Emissions}

Separate sampling during sootblowing and nonsootblowing periods was conducted at two sites: Baldwin Station (cyclone furnace/ESP) and Cardinal Station (dry-bottom, opposed wall-fired furnace/ESP). Differences between sootblowing and nonsootblowing trace metal concentrations for both sites were not statistically significant at the $95 \%$ confidence interval. Because the Baldwin Station data resulted from duct traverses conducted after the Cardinal Station tests, they are believed to be more representative of actual sootblowing effects. In general, Baldwin Station showed a slight enrichment of trace elements, greater total particulate, and higher emission factors, while the Cardinal Station showed a slight depletion of trace elements, less total particulate, and lower emission factors. 


\subsubsection{HAPs on Particle Surfaces}

A study of HAPs on particle surfaces at Plant Yates indicated that some trace elements, particularly Mo, Cd, and $\mathrm{Sb}$, are likely to be surface-enriched. These results are based on comparisons of flue gas particulate samples collected at the ESP inlet and outlet. Results for the stack gas particulate matter collected at Plant Yates were less conclusive because of apparent interaction of the fly ash with the JBR slurry. 


\subsection{REFERENCES}

1. Lee, B. "Highlights of the Clean Air Act Amendments of 1990," J. Air Waste Manage. Assoc. 1991, 41 (1), 16-19.

2. Bureau of National Affairs. "100 Guide to the Law," In Air Pollution Control: BNA Policy and Practice Series; Bureau of National Affairs: Washington DC, 1994; pp 100:101-600.

3. Chow, W.; Miller, M.J.; Torrens, I.M. "Pathways of Trace Elements in Power Plants: Interim Research Results and Implications," Fuel Processing Technology 1994, 39, 5-20.

4. Chu, P.; Nott, B.; Chow, W. "Results and Issues from the PISCES Field Tests," In Proceedings of the 2nd International Conference on Managing Hazardous Air Pollutants; Washington, DC, July 13-15, 1993; pp II-67-II-92.

5. Behrens, G.P.; Chu, P.; Chow, W.; Roberson, R.L. "A Method for Estimating Emissions of Trace Substances from Power Plants," Presented at the 87th Annual Meeting and Exhibition of the Air \& Waste Management Association, Cincinnati, $\mathrm{OH}$, June 19-24, 1994.

6. Chu, P.; McDannel, M.; Behrens, G. "PISCES Field Chemical Emission Measurement Program: Recent Emission Results form Oil-Fired Power Plants," Presented at the 87th Annual Meeting and Exhibition of the Air \& Waste Management Association, Cincinnati, OH, June 19-24, 1994.

7. Electric Power Research Institute. "Electric Utility Trace Substances Synthesis Report," Report No. TR-104614, Nov. 1994; Vols. 1-4.

8. Ohio Power Company. "HAP Sampling at Tidd PFBC," In Proceedings of the CoalFired Power Systems 94-Advances in IGCC and PFBC Review Meeting; McDaniel, H.M.; Staubly, R.K.; Venkataraman, V.K., Eds.; U.S. Department of Energy Office of Fossil Energy: Morgantown, WV, June 1994; Vol. 2.

9. Radian Corporation. "Hazardous Air Pollutant Sampling for Advanced Power Systems," In Proceedings of the Coal-Fired Power Systems 94-Advances in IGCC and PFBC Review Meeting; McDaniel, H.M.; Staubly, R.K.; Venkataraman, V.K., Eds.; U.S. Department of Energy Office of Fossil Energy: Morgantown, WV, June 1994; Vol. 2.

10. Radian Corporation. "A Study of Hazardous Air Pollutants at the Tidd PFBC Demonstration Plant," final report DCN 94-633-021-03 for American Electric Power Service Corporation; Oct. 27, 1994; Vol. 1.

11. Battelle. "A Study of Toxic Emissions from a Coal-Fired Power Plant - Niles Station Boiler No. 2," final report for U.S. DOE DE-AC22-93PC93251; June 1994, Vol. 1 of 2. 
12. Battelle. "A Study of Toxic Emissions from a Coal-Fired Power Plant Utilizing an ESP/Wet FGD System," final report for U.S. DOE DE-AC22-93PC93251; July 1994, Vol. 1 of 2.

13. Battelle. "A Study of Toxic Emissions from a Coal-Fired Power Plant Utilizing the SNOX Innovative Clean Coal Technology Demonstration," final report for U.S. DOE DE-AC22-93PC93251; July 1994, Vol. 1 of 2.

14. Radian Corporation. "A Study of Toxic Emissions from a Coal-Fired Power Plant Utilizing an ESP While Demonstrating the ICCT CT-121 FGD Project, " final report for U.S. DOE DE-AC22-93PC93253; June 16, 1994.

15. Roy F. Weston, Inc. "Toxics Assessment Report: Illinois Power Company Baldwin Power Station - Unit 2, Baldwin, Illinois," report for U.S. DOE DE-AC22-93PC93255; July 1994, Vol. 1, Sections 1-7.

16. Roy F. Weston, Inc. "Toxics Assessment Report: Minnesota Power Company Boswell Energy Center - Unit 2, Cohasset, Minnesota," report for U.S. DOE DE-AC22-93PC93255; July 1994, Vol. I, Sections 1-7.

17. Southern Research Institute. "Springerville Generating Station Unit No. 2," final report for U.S. DOE DE-AC22-93PC93254; SRI Report No. SRI-ENV-94-476-7960, June 1994.

18. Southern Research Institute. "Characterizing Toxic Emissions from a Coal-Fired Power Plant Demonstrating the AFGD ICCT Project and a Plant Utilizing a Dry Scrubber/Baghouse System: Bailly Station Units 7 and 8 and AFGD ICCT Project," final report for U.S. DOE DE-AC22-93PC93254; SRI Report No. SRI-ENV-94-6157960, Aug. 1994.

19. Energy and Environmental Research Corporation. "Assessment of Toxic Emissions from a Coal Fired Power Plant Utilizing an ESP," final report for U.S. DOE DEAC52-93PC93252; Dec. 1994.

20. Lengyel, J. Jr.; Obermiller, E.L. "Interlaboratory Variability and Accuracy in Trace Element Analyses of Coal," In Proceedings of the 4th Annual Pittsburgh Coal Conference; Pittsburgh, PA, 1987, pp 148-159.

21. Rosendale, L.W.; DeVito, M.S. "Interlaboratory Variability and Accuracy of Coal Analyses in the U.S. Department of Energy Utility Air Toxics Assessment Program," CONSOL INC: Library, PA, 1994; $41 \mathrm{p}$.

22. American Society for Testing and Materials. ASTM Volume 05.05: Gaseous Fuels, Coal, and Coke. ASTM: Philadelphia, PA, 1993.

23. Benson, S.A.; Hurley, J.P.; Zygarlicke, C.J. "Studies on Calcium-Based Deposition in Utility Boilers," In Proceedings of the Conference on Effects of Coal Quality on Power Plants; St. Louis, MO, Sept. 1990. 
24. Benson, S.A.; Zygarlicke, C.J.; Jones, M.L. "An Overview of Ash Deposition During the Combustion of Western U.S. Coals," In Proceedings of the Conference on Effects of Coal Quality on Power Plants; St. Louis, MO, Sept. 1990.

25. Clark, L.B.; Sloss, L.L. "Trace Element Emissions from Coal Combustion and Gasification," IEA Coal Research Report; 1992.

26. Ratafia-Brown, J.A. "Overview of Trace Element Partitioning in Flames and Furnaces of Utility Coal-Fired Boilers," In Proceedings of Trace Elements Workshop on Trace Element Transformations in Coal-Fired Power Systems; Scottsdale, AZ, April 19-22, 1993.

27. Benson, S.A.; Steadman, E.N.; Mehta, A.K.; Schmidt, C.E. "Trace Element Transformations in Coal-Fired Power Systems: Workshop Findings," In Proceedings of the 2nd International Conference on Managing Hazardous Air Pollutants; Chow, W., Levin, L. Eds.; Washington, DC, July 13-15, 1993; Electric Power Research Institute, EPRI TR-104295, Sept. 1994, pp III-119-135.

28. Meij, R. "The Fate of Trace Elements at Coal-Fired Power Plants," In Proceedings of the 2nd International Conference on Managing Hazardous Air Pollutants; Washington, DC; July 13-15, 1993 Chow, W., Levin, L., Eds.; Electric Power Research Institute, EPRI TR-104295, Sept. 1994; pp V-83-105.

29. Benson, S.A.; Hurley, J.P.; Zygarlicke, C.J.; Steadman, E.N.; Erickson, T.A. "Predicting Ash Behavior in Utility Boilers: Assessment of Current Status," Presented at the Conference on Effects of Coal Quality on Power Plants, Electric Power Research Institute, San Diego, CA, Aug. 1992.

30. Zygarlicke, C.J.; Benson, S.A.; Borio, R.W. "Pilot- and Bench-Scale Combustion Testing of a Wyoming Subbituminous/Oklahoma Bituminous Coal Blend," In CoalBlending and Switching of Low-Sulfur Western Fuels; Bryers, R.W.; Harding, N.S., Eds.; American Society of Mechanical Engineers: New York; 1994; pp 281-300.

31. Katrinak, K.A.; Zygarlicke, C.J. "Size-Related Variations in Coal Fly Ash Composition as Determined Using Automated Scanning Electron Microscopy," Prepr. Pap.-Am. Chem. Soc., Div. Fuel Chem. 1993, 38 (4), 1203-1209.

32. Bureau of National Affairs. "100 Guide to the Law," In Air Pollution Control: BNA Policy and Practice Series; Bureau of National Affairs: Washington DC, 1994; pp 100:101-600.

33. Kelly, T.J.; Ramamurthi, R.; Gordon, S.M.; Hays, M.J. "Ambient Measurement Methods and Properties of the 189 Title III Hazardous Air Pollutants," final report to U.S. Environmental Protection Agency; Batelle Press: Columbus, $\mathrm{OH}$, Oct. 1994, EPA-600/R-94-098.

34. Kelly, T.J.; Ramamurthi, M.; Pollack, A.J., Spicer, C.W. "Ambient Concentration Summaries for Clean Air Act Title III Hazardous Air Pollutants," final report for U.S. Environmental Protection Agency Contract No. 68-D80082; Battelle Report, July 1993. 
35. Natrella, M.G. "The Treatment of Outliers," In Experimental Statistics; National Bureau of Standards Handbook 91, Chapter 17; Superintendent of Documents, U.S. Government Printing Office: Washington, DC, 1963.

36. Shah, J.J.; Heyerdahl, E.K. "National Ambient Volatile Organic Compounds (VOCs) Database Update," Project Report EPA/600/3-88/010(a); U.S.

Environmental Protection Agency Contract No. 68-02-4190, Feb. 1988.

37. McDonald, J.R.; Dean, A.H. "A Manual for the Use of Electrostatic Precipitators to Collect Fly Ash Particles," Southern Research Institute report to the U.S. Environmental Protection Agency, EPA-600/8-80-025; May 1980.

38. 40 Code of Federal Regulations 266, Subpart H, Appendix IX. "Methods Manual for Compliance with the BIF Regulations,"; Section 3.0, "Sampling and Analytical Methods,"; Subsection 3.2, "Determination of Hexavalent Chromium Emissions from Stationary Sources" (Method Cr 6+1; July 1, 1991 edition.

39. Steinsberger S.C.; DeWees, W.G.; Bell, A.C.; Finken, R.A.; Knoll, J.E.; Midgett, M.R. "Development and Validation of the US EPA Hexavalent Chromium Source Emissions Measurement Method,". Fuel Processing Technology 1994, 39 (13).

40. Hassett, D.J.; Pflughoeft-Hassett, D.F.; McCarthy, G.J. "The Synthesis of Substituted Ettringites: Implications for Disposal of Hazardous Materials," Abstract presented at Joint Meeting of FACSS XVIII and Pacific Conference, Anaheim, CA, 1991.

41. Erickson, T.A.; Benson, S.A. "Trace Element Behavior in Gasification Systems," Presented at the 205th American Chemical Society National Meeting, Denver, CO, March 28 - April 2, 1993. 
APPENDIX A

INORGANIC DATA FOR STACK GAS SAMPLES 
Table A-1. Total Antimony

\begin{tabular}{|c|c|c|c|c|c|c|c|c|c|c|c|c|}
\hline \multirow{2}{*}{$\begin{array}{l}\text { Station/ } \\
\text { Plant } \\
\text { Name }\end{array}$} & \multirow{2}{*}{$\begin{array}{l}\text { Total } \\
\text { Stack } \\
\text { Conc... } \\
\mu \mathrm{g} / \mathrm{Nm}^{3}\end{array}$} & \multirow{2}{*}{$\begin{array}{l}\text { Total } \\
\text { Mean } \\
\text { Conc.. } \\
\mu \mathrm{g} / \mathrm{Nm}^{3}\end{array}$} & \multirow[b]{2}{*}{$\begin{array}{l}\text { Standard } \\
\text { Deviation }\end{array}$} & \multirow{2}{*}{$\begin{array}{l}\text { Blank } \\
\text { Conc. } \\
\text { Range, } \\
\mu g / \mathrm{Nm}^{3}\end{array}$} & \multicolumn{2}{|c|}{$\begin{array}{c}\text { M29 Internal Spike Recovery, } \\
\%\end{array}$} & \multirow{2}{*}{$\begin{array}{c}\text { M29 } \\
\text { Audit Spike } \\
\text { Recovery, } \\
\%\end{array}$} & \multirow[b]{2}{*}{$\begin{array}{c}\text { Above } \\
\text { DL }\end{array}$} & \multirow[b]{2}{*}{$\begin{array}{c}\text { Above } \\
\text { LLO }\end{array}$} & \multirow{2}{*}{$\begin{array}{l}\text { Emission } \\
\text { Factor, } \\
\text { Ib } / 10^{12} \text { Btu }\end{array}$} & \multirow{2}{*}{$\begin{array}{l}\text { Emission } \\
\text { Factor } \\
95 \% \mathrm{Cl}\end{array}$} & \multirow{2}{*}{$\begin{array}{c}\text { Control } \\
\text { Device } \\
\text { Removal, } \\
\%\end{array}$} \\
\hline & & & & & Fitter & Peroxide & & & & & & \\
\hline $\begin{array}{l}\text { Bailly } \\
\text { Station, } \\
\text { M29 }\end{array}$ & $\begin{array}{l}1.04 \\
0.09 \\
0.03\end{array}$ & 0.38 & 0.56 & $F$ & $\begin{array}{c}115 \\
126 \\
F\end{array}$ & $\begin{array}{c}86.7 \\
93 \\
P\end{array}$ & NA & NA & NA & 0.28 & 1.0 & $\begin{array}{l}\text { ESP\#7 } 98.83 \\
\text { ESP\#8 } 99.97 \\
\text { AFGD }-57.5\end{array}$ \\
\hline $\begin{array}{c}\text { Baldwin } \\
\text { Power Station, } \\
\text { M29 } \\
\text { Non-Sootblowing }\end{array}$ & $\begin{array}{l}2 \\
2 \\
2\end{array}$ & 2 & 0 & $\mathbf{P}$ & $\underset{P}{85-105}$ & $\stackrel{74-88}{P}$ & NA & $\mathbf{P}$ & $\mathrm{F}$ & 1.5 & 0.42 & ESP 90 \\
\hline $\begin{array}{c}\text { Baldwin } \\
\text { Power Station, } \\
\text { M29 } \\
\text { Sootblowing }\end{array}$ & $\begin{array}{l}\mathbf{3} \\
\mathbf{3} \\
\mathbf{3}\end{array}$ & 3 & 0 & $\mathbf{P}$ & $\begin{array}{c}85-105 \\
P\end{array}$ & $\stackrel{74-88}{P}$ & NA & $\mathbf{P}$ & $\mathrm{F}$ & 2.1 & 0.57 & ESP 95 \\
\hline $\begin{array}{c}\text { Boswell } \\
\text { Energy Center, } \\
\text { M29 }\end{array}$ & $\begin{array}{l}N D<0.42 \\
N D<0.43 \\
N D<1.86\end{array}$ & $N D<0.90$ & 0.83 & $\mathbf{P}$ & $\begin{array}{c}104 \\
P\end{array}$ & $\begin{array}{c}101 \\
P\end{array}$ & $\mathrm{NA}$ & $\mathbf{F}$ & $\mathbf{F}$ & $N D<0.68$ & 1.5 & $F F>97$ \\
\hline $\begin{array}{c}\text { Cardinal } \\
\text { Plant, } \\
\text { M29 } \\
\text { Non-Sootblowing }\end{array}$ & $\begin{array}{l}3.15 \\
5.13 \\
1.69 \\
2.26\end{array}$ & 3.06 & 1.51 & $\mathbf{F}$ & $\begin{array}{l}85 \\
P\end{array}$ & $\begin{array}{l}82 \\
P\end{array}$ & NA & NA & NA & 2.4 & 2.0 & \\
\hline $\begin{array}{l}\text { Cardinal Plant, } \\
\text { M29 } \\
\text { Sootblowing }\end{array}$ & $\begin{array}{c}3.21 \\
2.14 \\
0.225\end{array}$ & 1.86 & 1.51 & $\mathrm{~F}$ & $\begin{array}{l}85 \\
P\end{array}$ & $\begin{array}{l}82 \\
P\end{array}$ & NA & NA & NA & 1.4 & 3.2 & \\
\hline $\begin{array}{l}\text { Coal Creek } \\
\text { Station, } \\
\text { M29 }\end{array}$ & $\begin{array}{l}0.305 \\
0.206 \\
0.230\end{array}$ & 0.247 & 0.052 & $\begin{array}{c}\text { ND }<0.144 \\
\text { ND }<4.32 \\
F\end{array}$ & $\begin{array}{l}92 \\
103 \\
P\end{array}$ & $\underset{P}{92-98}$ & NA & $\mathbf{F}$ & $\mathrm{F}$ & 0.18 & 0.09 & $\begin{array}{l}\text { ESPF1 } 97.9 \\
\text { Wet FGD } 76.1 \\
\text { Combined } 99.6\end{array}$ \\
\hline $\begin{array}{l}\text { Niles } \\
\text { Station, } \\
\text { M29 }\end{array}$ & $\begin{array}{l}\text { ND }<0.59 \\
\text { ND }<0.60 \\
\text { ND }<0.61\end{array}$ & $N D<0.60$ & 0.01 & $\begin{array}{c}N D<0.689 \\
P\end{array}$ & \multicolumn{2}{|c|}{$\begin{array}{c}83-119 \\
P\end{array}$} & NA & $F$ & $F$ & $N D<0.36$ & 0.36 & ESP 99.8 \\
\hline $\begin{array}{l}\text { Niles/SNOX } \\
\text { Station, } \\
\text { M29 }\end{array}$ & $\begin{array}{l}N D<0.637 \\
N D<0.683 \\
N D<0.750\end{array}$ & ND $<0.69$ & 0.057 & $\underset{P}{N D}<0.575$ & \multicolumn{2}{|c|}{$\stackrel{83-119}{P}$} & NA & $F$ & $F$ & ND $<0.50$ & 0.50 & $\begin{array}{l}\text { FF } 99.74 \\
\text { SNOX } 99.61\end{array}$ \\
\hline $\begin{array}{l}\text { Plant } \\
\text { Yates, } \\
\text { M29 }\end{array}$ & $\begin{array}{l}0.13^{x} \\
0.06 \\
0.07\end{array}$ & 0.065 & 0.0070 & $\begin{array}{l}1.78 \\
F\end{array}$ & 84 & $\begin{array}{c}101 \\
P\end{array}$ & NA & $\mathbf{P}$ & $\mathbf{P}$ & 0.06 & 0.06 & $\begin{array}{l}\text { ESP } 98.8 \\
\text { JBR } 84.1\end{array}$ \\
\hline $\begin{array}{l}\text { Springerville } \\
\text { Generating } \\
\text { Station, } \\
\text { M29 }\end{array}$ & $\begin{array}{c}0.05 \\
0.12 \\
N D<0.01\end{array}$ & 0.058 & 0.058 & NA & $\begin{array}{c}68, \\
68 \\
F\end{array}$ & $\begin{array}{c}92, \\
91 \\
\mathrm{p}\end{array}$ & NA & NA & NA & 0.04 & 0.095 & $\begin{array}{c}\text { FF } 99.7 \\
\text { SDA-FF } 99.3\end{array}$ \\
\hline
\end{tabular}

NA - Not available. ND - Nondetect. NR - Not required. P - Pass. F - Fail. $x$ - Value invalid because of high background level.

$y$-Value considered an outlier. Note: A detailed discussion of $Q A / Q C$ criteria is presented in Section 4.2.1. 


\begin{tabular}{|c|c|c|c|c|c|c|c|c|c|c|c|c|c|}
\hline \multirow{2}{*}{$\begin{array}{l}\text { Station/ } \\
\text { Plant } \\
\text { Name }\end{array}$} & \multirow{2}{*}{$\begin{array}{c}\text { Total } \\
\text { Stack } \\
\text { Conc., } \\
\mu \mathrm{g} / \mathrm{Nm}^{3}\end{array}$} & \multirow{2}{*}{$\begin{array}{c}\text { Total } \\
\text { Mean } \\
\text { Conc.. } \\
\mu \mathrm{g} / \mathrm{Nm}^{3} \\
\end{array}$} & \multirow[b]{2}{*}{$\begin{array}{l}\text { Standard } \\
\text { Deviation }\end{array}$} & \multirow{2}{*}{$\begin{array}{l}\text { Blank } \\
\text { Conc. } \\
\text { Range, } \\
\mu g / \mathrm{Nm}^{3} \\
\end{array}$} & \multicolumn{2}{|c|}{$\begin{array}{c}\text { M29 internal Spike Recovery, } \\
\%\end{array}$} & \multicolumn{2}{|c|}{$\begin{array}{l}\text { M29 Audit Spike Recovery, } \\
\%\end{array}$} & \multirow[b]{2}{*}{$\begin{array}{l}\text { Above } \\
\text { DL }\end{array}$} & \multirow[b]{2}{*}{$\begin{array}{c}\text { Above } \\
\text { LLO }\end{array}$} & \multirow{2}{*}{$\begin{array}{l}\text { Emission } \\
\text { Factor, } \\
\mid \mathrm{b} / 10^{12} \mathrm{Btu}\end{array}$} & \multirow{2}{*}{$\begin{array}{l}\text { Emission } \\
\text { Factor } \\
95 \% \mathrm{Cl} \\
\end{array}$} & \multirow{2}{*}{$\begin{array}{c}\text { Control } \\
\text { Device } \\
\text { Removal, } \\
\% \\
\end{array}$} \\
\hline & & & & & Filter & Peroxide & Filter & Peroxide & & & & & \\
\hline $\begin{array}{c}\text { Bailly } \\
\text { Station, } \\
\text { M29 } \\
\end{array}$ & $\begin{array}{l}5.18 \\
0.57 \\
0.42 \\
\end{array}$ & 1.43 & 1.79 & $p$ & $\begin{array}{c}28 \\
38 \\
F \\
\end{array}$ & $\begin{array}{c}86, \\
86.8 \\
P \\
\end{array}$ & $\begin{array}{c}18 \\
27 \\
\mathrm{~F} \\
\end{array}$ & $\begin{array}{c}85 \\
50 \\
\mathrm{~F} \\
\end{array}$ & NA & NA & 1.1 & 3.3 & $\begin{array}{c}\text { ESP\#7 >95.05 } \\
\text { ESP\#8 } 98.41 \\
\text { AFGD } 58.26 \pm 41 \\
\end{array}$ \\
\hline $\begin{array}{c}\text { Baldwin } \\
\text { Power Station, } \\
\text { M29 } \\
\text { Non-Sootblowing } \\
\end{array}$ & $\begin{array}{r}9 \\
10 \\
36 \\
\end{array}$ & 18.3 & 15 & $p$ & $\begin{array}{c}76-101 \\
F\end{array}$ & $\begin{array}{c}9-36 \\
P \\
\end{array}$ & $\begin{array}{c}407 \\
102 \\
F \\
\end{array}$ & $\begin{array}{l}39 . \\
64 \\
F \\
\end{array}$ & $p$ & $P$ & 13 & 28 & ESP 99 \\
\hline $\begin{array}{c}\text { Baldwin } \\
\text { Power Station, } \\
\text { M29 } \\
\text { Sootblowing } \\
\end{array}$ & $\begin{array}{l}15 \\
15 \\
20 \\
\end{array}$ & 17 & 3 & $p$ & $\begin{array}{c}76-101 \\
F\end{array}$ & $\begin{array}{c}9-36 \\
P \\
\end{array}$ & $\begin{array}{c}407 \\
102 \\
\mathrm{~F} \\
\end{array}$ & $\begin{array}{c}39 \\
64 \\
F \\
\end{array}$ & $p$ & $P$ & 12 & 6.5 & ESP 99 \\
\hline $\begin{array}{c}\text { Boswell } \\
\text { Energy Center, } \\
\text { M29 } \\
\end{array}$ & $\begin{array}{l}N D<0.44 \\
N D<0.43 \\
N D<0.44 \\
\end{array}$ & NO $<0.29$ & 0.13 & $p$ & $\begin{array}{c}98.4 \\
P\end{array}$ & $\begin{array}{c}88.0 \\
p\end{array}$ & $\begin{array}{c}86 \\
99 \\
P \\
\end{array}$ & $\begin{array}{c}64 \\
107 \\
F \\
\end{array}$ & $F$ & $F$ & $\mathrm{ND}<0.32$ & 0.32 & $F F>99$ \\
\hline $\begin{array}{c}\text { Cardinal } \\
\text { Plant, } \\
\text { M29 } \\
\text { Non-Sootblowing } \\
\end{array}$ & $\begin{array}{r}4.7 \\
6.12 \\
3.62 \\
3.53 \\
\end{array}$ & 4.49 & 1.21 & $P$ & $\mathrm{NA}$ & NA & $\begin{array}{r}85 \\
72 \\
P \\
\end{array}$ & $\begin{array}{l}80 \\
P \\
\end{array}$ & $P$ & $P$ & 3.5 & 2.0 & \multirow{2}{*}{ ESP 99.67} \\
\hline \begin{tabular}{c|} 
Cardinal \\
Plant, \\
M29 \\
Sootblowing \\
\end{tabular} & $\begin{array}{l}1.73 \\
3.18 \\
1.73 \\
\end{array}$ & 2.21 & 0.84 & $\mathrm{P}$ & NA & $\mathrm{NA}$ & $\begin{array}{r}85 \\
72 \\
P \\
\end{array}$ & $\begin{array}{l}80 \\
P \\
\end{array}$ & $p$ & $p$ & 1.7 & 1.8 & \\
\hline $\begin{array}{l}\text { Coal Creek } \\
\text { Station, } \\
\text { M29 } \\
\end{array}$ & $\begin{array}{l}2.00 \\
1.61 \\
1.36 \\
\end{array}$ & 1.66 & 0.322 & $\begin{array}{c}0.222 \\
6.63 \\
F \\
\end{array}$ & $\begin{array}{c}92- \\
101 \\
P \\
\end{array}$ & $\begin{array}{c}95- \\
103 \\
P \\
\end{array}$ & $\begin{array}{l}73 \\
88 \\
P \\
\end{array}$ & NA & $\mathrm{P}$ & $P$ & 1.2 & 0.58 & $\begin{array}{c}\text { ESP\#1 } 99.9 \\
\text { Wet FGD } 29.1 \\
\text { Combined } 99.9 \\
\end{array}$ \\
\hline $\begin{array}{c}\text { Coal Creek } \\
\text { Station, } \\
\text { HEST } \\
\end{array}$ & $\begin{array}{l}\text { ND }<0.012 \\
\text { ND }<0.010 \\
\text { ND }<0.012 \\
\end{array}$ & $\mathrm{ND}<0.011$ & 0.001 & NA & $\mathrm{NR}$ & $\mathrm{NR}$ & NR & $\mathrm{NR}$ & $\mathrm{NR}$ & NA & $\mathrm{NA}$ & $\mathrm{NA}$ & NA \\
\hline $\begin{array}{c}\text { Niles } \\
\text { Station, } \\
\text { M29 }\end{array}$ & $\begin{array}{r}79.4 \\
59.6 \\
70.3 \\
\end{array}$ & 69.8 & 9.91 & $\begin{array}{c}2.69 \\
P\end{array}$ & \multicolumn{2}{|c|}{$\begin{array}{c}76-115 \\
P \\
\end{array}$} & $\begin{array}{c}89 . \\
94 \\
\mathrm{P} \\
\end{array}$ & $\begin{array}{c}77 \\
77 \\
P \\
\end{array}$ & $\mathrm{P}$ & $P$ & 42 & 19 & ESP 97.41 \\
\hline $\begin{array}{c}\text { Niles } \\
\text { Station, } \\
\text { HEST } \\
\end{array}$ & $\begin{array}{l}74.5 \\
82.2 \\
47.5 \\
\end{array}$ & 68.1 & 18.2 & NA & $\mathrm{NR}$ & NR & $\mathrm{NR}$ & $\mathrm{NR}$ & NA & NA & NA & NA & NA \\
\hline $\begin{array}{c}\text { Niles/SNOX } \\
\text { Station, } \\
\text { M29 } \\
\end{array}$ & $\begin{array}{l}N D<0.637 \\
N D<0.683 \\
N D<0.75 \\
\end{array}$ & NO $<0.69$ & $N D<0.057$ & $\begin{array}{c}\mathrm{ND}<0.575 \\
\mathrm{P}\end{array}$ & \multicolumn{2}{|c|}{$\begin{array}{c}76-115 \\
P\end{array}$} & $\begin{array}{r}89, \\
94 \\
\mathrm{P} \\
\end{array}$ & $\begin{array}{r}77 \\
77 \\
\mathrm{P} \\
\end{array}$ & $\mathrm{F}$ & $F$ & $N D<0.50$ & 0.50 & $\begin{array}{c}\text { FF } 99.61 \\
\text { SNOX } 99.99 \\
\end{array}$ \\
\hline $\begin{array}{l}\text { Niles/SNOX } \\
\text { Station, } \\
\text { HEST }\end{array}$ & $\begin{array}{l}\text { ND }<0.010 \\
\text { ND }<0.124\end{array}$ & ND $<0.11$ & 0.017 & NA & NR & NR & NR & NR & NA & NA & NA & NA & NA \\
\hline $\begin{array}{c}\text { Plant } \\
\text { Yates, } \\
\text { M29 } \\
\end{array}$ & $\begin{array}{l}1.13 \\
1.13 \\
1.30 \\
\end{array}$ & 1.19 & 0.10 & $\begin{array}{c}N D<0.179 \\
\quad P \\
\end{array}$ & $\mathrm{NA}$ & $\begin{array}{c}100 \\
P\end{array}$ & $\begin{array}{c}211, \\
112 \\
\mathrm{~F} \\
\end{array}$ & $\begin{array}{c}100.2 \\
104.2 \\
P \\
\end{array}$ & $P$ & $P$ & 1.2 & 0.2 & $\begin{array}{l}\text { ESP } 95.9 \\
\text { JBR } 92.7 \\
\end{array}$ \\
\hline $\begin{array}{c}\text { Springerville } \\
\text { Generating } \\
\text { Station, } \\
\text { M29 }\end{array}$ & $\begin{array}{c}0.62 \\
N D<0.01 \\
N D<0.01\end{array}$ & 0.21 & 0.36 & NA & NA & $\begin{array}{c}94 \\
\mathrm{P} \\
\end{array}$ & $\begin{array}{c}97.2 \\
137.5 \\
F\end{array}$ & $\begin{array}{c}80.9 \\
70.7 \\
p\end{array}$ & NA & NA & 0.14 & 0.60 & $\begin{array}{l}\text { FF } 99.97 \\
\text { SDA-FF } 99.99\end{array}$ \\
\hline
\end{tabular}

NA - Not available. ND - Nondetect. NR - Not required. P - Pass. F - Fail. $x$ - Value invalid because of high background level.

$y$-Value considered an outlier. Note: A detailed discussion of QA/QC criteria is presented in Section 4.2.1. 
Table A-3. Total Barium

\begin{tabular}{|c|c|c|c|c|c|c|c|c|c|c|c|c|}
\hline \multirow{2}{*}{$\begin{array}{l}\text { Station/ } \\
\text { Plant } \\
\text { Name }\end{array}$} & \multirow{2}{*}{$\begin{array}{l}\text { Total } \\
\text { Stack } \\
\text { Conc., } \\
\mu \mathrm{g} / \mathrm{Nm}^{3}\end{array}$} & \multirow{2}{*}{$\begin{array}{c}\text { Total } \\
\text { Mean } \\
\text { Conc., } \\
\mu \mathrm{g} / \mathrm{Nm}^{3}\end{array}$} & \multirow[b]{2}{*}{$\begin{array}{l}\text { Standard } \\
\text { Deviation }\end{array}$} & \multirow{2}{*}{$\begin{array}{l}\text { Blank } \\
\text { Conc. } \\
\text { Range, } \\
\mu \mathrm{g} / \mathrm{Nm}^{3}\end{array}$} & \multicolumn{2}{|c|}{ M29 Internal Spike Recovery, \% } & \multirow{2}{*}{$\begin{array}{c}\text { M29 } \\
\text { Audit Spike } \\
\text { Recovery. } \\
\%\end{array}$} & \multirow[b]{2}{*}{$\begin{array}{c}\text { Above } \\
\text { DL }\end{array}$} & \multirow[b]{2}{*}{$\begin{array}{l}\text { Above } \\
\text { LLa }\end{array}$} & \multirow{2}{*}{$\begin{array}{l}\text { Emission } \\
\text { Factor, } \\
\text { Ib/10 } 10^{12} \text { Btu }\end{array}$} & \multirow{2}{*}{$\begin{array}{l}\text { Emission } \\
\text { Factor } \\
95 \% \mathrm{Cl}\end{array}$} & \multirow{2}{*}{$\begin{array}{c}\text { Control } \\
\text { Device } \\
\text { Removal, } \\
\%\end{array}$} \\
\hline & & & & & Filter & Peroxide & & & & & & \\
\hline $\begin{array}{l}\text { Bailly } \\
\text { Station, } \\
\text { M29 }\end{array}$ & $\begin{array}{l}1.99 \\
1.36 \\
2.07\end{array}$ & 1.71 & 0.39 & $\mathbf{F}$ & $\begin{array}{c}36 \\
100 \\
F\end{array}$ & $\begin{array}{l}94, \\
99 \\
\mathrm{P}\end{array}$ & NA & NA & NA & 1.3 & 0.72 & $\begin{array}{c}\text { ESP \#7.98.75 } \\
\text { ESP \#899.7 } \\
\text { AFGO } 89.18\end{array}$ \\
\hline $\begin{array}{c}\text { Baldwin } \\
\text { Power Station, } \\
\text { M29 } \\
\text { Non-Sootblowing }\end{array}$ & $\begin{array}{l}9 \\
7 \\
6\end{array}$ & 7 & 2 & $\mathbf{P}$ & NA & NA & NA & $\mathbf{P}$ & $\mathbf{P}$ & 5.3 & 2.9 & $E S P>99$ \\
\hline $\begin{array}{l}\text { Baldwin } \\
\text { Power Station, } \\
\text { M29 } \\
\text { Soot Blow }\end{array}$ & $\begin{array}{l}6 \\
5 \\
3\end{array}$ & 5 & 2 & $\mathbf{P}$ & NA & NA & NA & $\mathbf{P}$ & $p$ & 3.4 & 2.4 & $E S P>99$ \\
\hline $\begin{array}{c}\text { Boswell } \\
\text { Energy Center. } \\
\text { M29 }\end{array}$ & $\begin{array}{l}92.57 \\
44.42 \\
188.81\end{array}$ & 108.60 & 4.69 & $\mathbf{P}$ & NA & NA & NA & $\mathrm{P}$ & $P$ & 82 & 140 & FF 98 \\
\hline $\begin{array}{c}\text { Cardinal } \\
\text { Plant, } \\
\text { M29 } \\
\text { Non-Sootblowing }\end{array}$ & $\begin{array}{l}1.10 \\
1.06 \\
1.07 \\
1.35\end{array}$ & 1.14 & 0.14 & $F$ & $\begin{array}{c}85 \\
P\end{array}$ & $\begin{array}{c}21 \\
F\end{array}$ & NA & NA & NA & 0.89 & 0.65 & \multirow{2}{*}{ ESP 99.84} \\
\hline $\begin{array}{c}\text { Cardinal Plant, } \\
\text { M29 } \\
\text { Sootblowing }\end{array}$ & $\begin{array}{l}0.514 \\
0.961 \\
0.778\end{array}$ & 0.751 & 0.225 & $\mathrm{~F}$ & $\begin{array}{c}85 \\
P\end{array}$ & $\begin{array}{c}21 \\
F\end{array}$ & NA & NA & NA & 0.59 & 0.64 & \\
\hline $\begin{array}{l}\text { Coal Creek } \\
\text { Station, } \\
\text { M29 }\end{array}$ & $\begin{array}{l}256 \\
204 \\
192\end{array}$ & 217 & 34.0 & $\begin{array}{c}N D<0.853 \\
N D<2.33 \\
P\end{array}$ & $\begin{array}{c}93- \\
103 \\
P\end{array}$ & $\begin{array}{c}92.5 \\
P\end{array}$ & NA & $P$ & $\mathbf{P}$ & 162 & 61 & $\begin{array}{c}\text { ESPH1 99.3 } \\
\text { Wet FGD 1.13.6) } \\
\text { Combined } 99.0\end{array}$ \\
\hline $\begin{array}{c}\text { Niles } \\
\text { Station, } \\
\text { M29 }\end{array}$ & $\begin{array}{l}15.4 \\
4.63 \\
6.45\end{array}$ & 8.83 & 5.76 & $\stackrel{12.8}{F}$ & \multicolumn{2}{|c|}{$\begin{array}{c}87-104 \\
P\end{array}$} & NA & $\mathbf{P}$ & $\mathbf{P}$ & 5.4 & 9.3 & ESP 99.34 \\
\hline $\begin{array}{l}\text { Niles/SNOX } \\
\text { Station, } \\
\text { M29 }\end{array}$ & $\begin{array}{c}0.609 \\
N D<0.094 \\
N D<0.105\end{array}$ & 0.236 & 0.322 & $\stackrel{15.9}{F}$ & \multicolumn{2}{|c|}{$\begin{array}{c}87-104 \\
P\end{array}$} & NA & $\mathrm{F}$ & $\mathrm{F}$ & 0.17 & 0.59 & $\begin{array}{c}\text { FF } 99.87 \\
\text { SNOX } 99.98\end{array}$ \\
\hline $\begin{array}{l}\text { Plant } \\
\text { Yates, } \\
\text { M29 }\end{array}$ & $\begin{array}{c}\text { NO }<1.49^{x} \\
3.72 \\
2.09\end{array}$ & 2.91 & 1.15 & $\begin{array}{c}0.734 \\
P\end{array}$ & $\begin{array}{c}75 \\
P\end{array}$ & $\stackrel{106}{p}$ & NA & $\mathbf{P}$ & $\mathbf{p}$ & 2.8 & 9.9 & $\begin{array}{l}\text { ESP } 98.3 \\
\text { JBR } 96.1\end{array}$ \\
\hline $\begin{array}{l}\text { Springerville } \\
\text { Generating } \\
\text { Station, } \\
\text { M29 }\end{array}$ & $\begin{array}{l}13.6 \\
19.9 \\
26.6\end{array}$ & 20.0 & 6.5 & NA & NA & $\begin{array}{l}96, \\
96 \\
P\end{array}$ & NA & NA & NA & 13 & 11 & $\begin{array}{c}\text { FF } 99.98 \\
\text { SDA-FF } 99.95\end{array}$ \\
\hline
\end{tabular}

NA - Not available. ND - Nondetect. NR - Not required. P - Pass. F - Fail. $X$ - Value invalid because of high background level.
$Y$ - Value considered an outlier. Note: A detailed discussion of QA/OC criteria is presented in Section 4.2.1. 
Table A-4. Total Beryllium

\begin{tabular}{|c|c|c|c|c|c|c|c|c|c|c|c|c|}
\hline \multirow{2}{*}{$\begin{array}{c}\text { Station/ } \\
\text { Plant } \\
\text { Name } \\
\end{array}$} & \multirow{2}{*}{$\begin{array}{c}\text { Total } \\
\text { Stack } \\
\text { Conc.., } \\
\mu \mathrm{g} / \mathrm{Nm}^{3} \\
\end{array}$} & \multirow{2}{*}{$\begin{array}{c}\text { Total } \\
\text { Mean } \\
\text { Conc.., } \\
\mu \mathrm{g} / \mathrm{Nm}^{3} \\
\end{array}$} & \multirow[b]{2}{*}{$\begin{array}{l}\text { Standard } \\
\text { Deviation }\end{array}$} & \multirow{2}{*}{$\begin{array}{c}\text { Blank } \\
\text { Conc. } \\
\text { Range, } \\
\mu \mathrm{g} / \mathrm{Nm}^{3} \\
\end{array}$} & \multicolumn{2}{|c|}{$\begin{array}{c}\text { M29 Internal Spike Recovery, } \\
\%\end{array}$} & \multirow{2}{*}{$\begin{array}{c}\text { M29 } \\
\text { Audit Spike } \\
\text { Recovery. } \\
\%\end{array}$} & \multirow[b]{2}{*}{$\begin{array}{c}\text { Above } \\
\text { OL }\end{array}$} & \multirow[b]{2}{*}{$\begin{array}{c}\text { Above } \\
\text { LLO }\end{array}$} & \multirow{2}{*}{$\begin{array}{c}\text { Emission } \\
\text { Factor, } \\
\text { |b/10/12 } \mathrm{Btu}\end{array}$} & \multirow{2}{*}{$\begin{array}{l}\text { Emission } \\
\text { Factor } \\
95 \% \mathrm{Cl}\end{array}$} & \multirow{2}{*}{$\begin{array}{c}\text { Control } \\
\text { Device } \\
\text { Removal, } \\
\% \\
\end{array}$} \\
\hline & & & & & Filter & Peroxide & & & & & & \\
\hline $\begin{array}{c}\text { Bailly } \\
\text { Station, } \\
\text { M29 }\end{array}$ & $\begin{array}{c}0.14 \\
0.07 \\
\text { ND }<0.10\end{array}$ & ND $<0.09$ & 0.05 & $\mathbf{P}$ & $\begin{array}{l}91, \\
98 \\
P\end{array}$ & $\begin{array}{c}97.2 \\
94.8 \\
P\end{array}$ & NA & NA & NA & ND $<0.07$ & 0.07 & $\begin{array}{l}\text { ESP\#7 98.58 } \\
\text { ESP\#8 99.92 } \\
\text { AFGD } 88.70\end{array}$ \\
\hline $\begin{array}{c}\text { Baldwin } \\
\text { Power Station, } \\
\text { M29 } \\
\text { Non-Sootblowing }\end{array}$ & $\begin{array}{l}2 \\
2 \\
2\end{array}$ & 2 & 0 & $\mathbf{P}$ & NA & NA & NA & $P$ & $\mathbf{P}$ & 1.4 & 0.24 & ESP 97 \\
\hline $\begin{array}{c}\text { Baldwin } \\
\text { Power Station, } \\
\text { M29 } \\
\text { Sootblowing }\end{array}$ & $\begin{array}{l}2 \\
3 \\
3\end{array}$ & 3 & 0.6 & $\mathbf{P}$ & NA & NA & NA & $\mathbf{P}$ & $\mathbf{P}$ & 1.7 & 0.86 & ESP 97 \\
\hline $\begin{array}{c}\text { Boswell } \\
\text { Energy Center, } \\
\text { M29 }\end{array}$ & $\begin{array}{l}N D<0.17 \\
\text { ND }<0.17 \\
\text { NO }<0.17\end{array}$ & ND $<0.17$ & 0 & $P$ & NA & NA & NA & $\boldsymbol{F}$ & $\boldsymbol{F}$ & $\mathrm{ND}<0.13$ & 0.13 & $F F>99$ \\
\hline $\begin{array}{c}\text { Cardinal } \\
\text { Plant, } \\
\text { M29 } \\
\text { Non-Sootblowing } \\
\end{array}$ & $\begin{array}{l}0.0763 \\
0.124 \\
0.072 \\
0.0895 \\
\end{array}$ & 0.090 & 0.0236 & $\mathbf{P}$ & $\begin{array}{c}74 \\
P\end{array}$ & $\begin{array}{c}98 \\
P\end{array}$ & NA & $\mathbf{P}$ & $F$ & 0.070 & 0.039 & ESP 9993 \\
\hline $\begin{array}{c}\text { Cardinal } \\
\text { Plant, } \\
\text { M29 } \\
\text { Sootblowing } \\
\end{array}$ & $\begin{array}{l}0.0284 \\
0.0665 \\
0.0509 \\
\end{array}$ & 0.0486 & 0.010 & $P$ & $\begin{array}{c}74 \\
P\end{array}$ & $\begin{array}{l}98 \\
\mathrm{P}\end{array}$ & NA & $P$ & $F$ & 0.038 & 0.042 & (6) \\
\hline $\begin{array}{c}\text { Niles } \\
\text { Station, } \\
\text { M29 }\end{array}$ & $\begin{array}{l}0.31 \\
0.33 \\
0.28\end{array}$ & 0.31 & 0.02 & $\begin{array}{c}N D<0.114 \\
P\end{array}$ & \multicolumn{2}{|c|}{$\begin{array}{c}72-108 \\
P\end{array}$} & NA & $\mathbf{P}$ & $\mathbf{F}$ & 0.19 & 0.05 & ESP 99.56 \\
\hline $\begin{array}{l}\text { Niles/SNOX } \\
\text { Station, } \\
\text { M29 }\end{array}$ & $\begin{array}{c}0.376 \\
0.247 \\
\mathrm{ND}<0.162 \\
\end{array}$ & 0.235 & 0.148 & $\begin{array}{c}N D<0.110 \\
P\end{array}$ & \multicolumn{2}{|c|}{$\begin{array}{c}72-108 \\
P\end{array}$} & NA & $\begin{array}{c}0.3 \\
F\end{array}$ & $\mathbf{F}$ & 0.17 & 0.27 & $\begin{array}{c}\text { FF } 99.93 \\
\text { SNOX } 99.69 \\
\end{array}$ \\
\hline $\begin{array}{l}\text { Plant } \\
\text { Yates, } \\
\text { M29 }\end{array}$ & $\begin{array}{c}\text { ND }<0.06^{*} \\
0.12 \\
0.08\end{array}$ & 0.10 & 0.03 & $\begin{array}{c}N D<0.15 \\
P\end{array}$ & $\begin{array}{c}89 \\
p\end{array}$ & $\begin{array}{c}108 \\
P\end{array}$ & NA & $\mathrm{F}$ & $F$ & 0.1 & 0.27 & $\begin{array}{l}\text { ESP } 98.1 \\
\text { JBR } 92.6\end{array}$ \\
\hline $\begin{array}{c}\text { Springerville } \\
\text { Generating } \\
\text { Station, } \\
\text { M29. } \\
\end{array}$ & $\begin{array}{l}N O<0.1 \\
N D<0.1 \\
N D<0.1 \\
\end{array}$ & $N D<0.1$ & NA & NA & $\begin{array}{c}101, \\
102 \\
\mathrm{P}\end{array}$ & $\begin{array}{c}89, \\
89 \\
\mathrm{P}\end{array}$ & NA & NA & $\mathrm{NA}$ & $N D<0.04$ & 0.04 & $\begin{array}{c}F F>99.98 \\
\text { SDA-FF } 99.96\end{array}$ \\
\hline
\end{tabular}

NA - Not available. ND - Nondetect. NR - Not required. P-Pass. F Fail. $x$ - Value invalid because of high background level.

$y$-Value considered an outlier. Note: A detailed discussion of $\mathrm{QA} / \mathrm{OC}$ criteria is presented in Section 4.2.1. 
Table A-5. Total Boron

\begin{tabular}{|c|c|c|c|c|c|c|c|c|c|c|c|c|}
\hline \multirow{2}{*}{$\begin{array}{l}\text { Station/ } \\
\text { Plant } \\
\text { Name }\end{array}$} & \multirow{2}{*}{$\begin{array}{c}\text { Total } \\
\text { Stack } \\
\text { Conc.., } \\
\mu \mathrm{g} / \mathrm{Nm}^{3}\end{array}$} & \multirow{2}{*}{$\begin{array}{c}\text { Total } \\
\text { Mean } \\
\text { Conc., } \\
\mu \mathrm{g} / \mathrm{Nm}^{3}\end{array}$} & \multirow[b]{2}{*}{$\begin{array}{l}\text { Standard } \\
\text { Deviation }\end{array}$} & \multirow{2}{*}{$\begin{array}{l}\text { Blank } \\
\text { Conc. } \\
\text { Range, } \\
\mu \mathrm{g} / \mathrm{Nm}^{3}\end{array}$} & \multicolumn{2}{|c|}{$\begin{array}{l}\text { M29 Internal Spike Recovery, } \\
\%\end{array}$} & \multirow{2}{*}{$\begin{array}{c}\text { M29 } \\
\text { Audit Spike } \\
\text { Recovery, } \\
\%\end{array}$} & \multirow[b]{2}{*}{$\begin{array}{c}\text { Above } \\
\text { DL }\end{array}$} & \multirow[b]{2}{*}{$\begin{array}{l}\text { Above } \\
\text { LLa }\end{array}$} & \multirow{2}{*}{$\begin{array}{l}\text { Emission } \\
\text { Factor, } \\
\text { Ib/10': } \\
\text { Btu }\end{array}$} & \multirow{2}{*}{$\begin{array}{l}\text { Emission } \\
\text { Factor } \\
95 \% \mathrm{Cl}\end{array}$} & \multirow{2}{*}{$\begin{array}{c}\text { Control } \\
\text { Device } \\
\text { Removal, } \\
\%\end{array}$} \\
\hline & & & & & Filter & Peroxide & & & & & & \\
\hline $\begin{array}{l}\text { Bailly } \\
\text { Station, } \\
\text { M29 }\end{array}$ & $\begin{array}{c}944 \\
1150 \\
1600\end{array}$ & 1230 & 340 & $\mathbf{P}$ & NA & $\begin{array}{c}422, \\
197 \\
F\end{array}$ & NA & NA & NA & 909 & 625 & $\begin{array}{c}\text { ESP\#7 } 21 \pm 26 \\
\text { ESP\#8 } 19 \pm 22 \\
\text { AFGD } 91.16\end{array}$ \\
\hline $\begin{array}{c}\text { Baldwin } \\
\text { Power Station, } \\
\text { M29 } \\
\text { Non-Sootblowing }\end{array}$ & $\begin{array}{c}11622 \\
10037 \\
9703\end{array}$ & 10454 & 1025 & $\begin{array}{c}\mathrm{F} 75900 \mu \mathrm{g} \\
\mathrm{FH}<45 \mu \mathrm{g} \\
\mathrm{BH}<270 \mu \mathrm{g}\end{array}$ & NA & NA & NA & NA & NA & 7700 & 1800 & ESP 80 \\
\hline $\begin{array}{c}\text { Baldwin } \\
\text { Power Station, } \\
\text { M29 } \\
\text { Sootblowing }\end{array}$ & $\begin{array}{l}12073 \\
12605 \\
9995\end{array}$ & 11558 & 1379 & $\begin{array}{l}\mathrm{F} 75900 \mu \mathrm{g} \\
\mathrm{FH}<45 \mu \mathrm{g} \\
\mathrm{BH}<270 \mu \mathrm{g}\end{array}$ & NA & NA & NA & NA & NA & 8600 & 1800 & ESP 85 \\
\hline $\begin{array}{c}\text { Boswell } \\
\text { Energy Center. } \\
\text { M29 }\end{array}$ & $\begin{array}{l}1581.5 \\
584.43 \\
230.21\end{array}$ & 798.71 & 700.67 & $\begin{array}{c}\mathrm{F} 54800 \mu \mathrm{g} \\
\mathrm{FH} 307 \mu \mathrm{g} \\
\mathrm{BH}<75 \mu \mathrm{g}\end{array}$ & NA & NA & NA & NA & NA & 610 & 1400 & FF 98 \\
\hline $\begin{array}{c}\text { Cardinal } \\
\text { Plant, } \\
\text { M29 } \\
\text { Non-Sootblowing }\end{array}$ & $\begin{array}{l}2110 \\
2710 \\
2710 \\
2333\end{array}$ & 2466 & 296.4 & $\begin{array}{l}16 \mu \mathrm{g}, \\
98 \mu \mathrm{g}\end{array}$ & $\begin{array}{l}87 \\
P\end{array}$ & NA & NA & NA & NA & 1910 & 812 & \multirow{2}{*}{ ESP 56.30} \\
\hline $\begin{array}{l}\text { Cardinal } \\
\text { Plant, } \\
\text { M29 } \\
\text { Sootblowing }\end{array}$ & $\begin{array}{l}1820 \\
2290 \\
2620\end{array}$ & 2243 & 402.0 & $\begin{array}{l}16 \mu \mathrm{g} \\
98 \mu \mathrm{g}\end{array}$ & $\stackrel{87}{P}$ & NA & NA & NA & NA & 1750 & 1191 & \\
\hline $\begin{array}{l}\text { Coal Creek } \\
\text { Station, } \\
\text { M29 }\end{array}$ & $\begin{array}{l}10.4 \\
55.8 \\
11.5\end{array}$ & 25.9 & 25.9 & $\begin{array}{c}N D<7.33 \\
N D<20 \\
P\end{array}$ & $\begin{array}{l}94 . \\
96 \\
P\end{array}$ & $\stackrel{92.0}{P}$ & NA & NA & NA & 19 & 49 & $\begin{array}{l}\text { ESP\#1 } 98.7 \\
\text { Wet FGO 71.0 } \\
\text { Combined } 99.8\end{array}$ \\
\hline $\begin{array}{l}\text { Niles } \\
\text { Station, } \\
\text { M29 }\end{array}$ & NA & NA & NA & NA & NA & NA & NA & NA & NA & NA & NA & NA \\
\hline $\begin{array}{l}\text { Niles/SNOX } \\
\text { Station, } \\
\text { M29 }\end{array}$ & NA & NA & NA & NA & NA & NA & NA & NA & NA & NA & NA & NA \\
\hline $\begin{array}{l}\text { Plant } \\
\text { Yates, } \\
\text { M29 }\end{array}$ & $\begin{array}{l}468 \\
412 \\
440\end{array}$ & 440 & 28 & NA & NA & $\begin{array}{c}104 \\
P\end{array}$ & NA & $\mathbf{P}$ & P & NA & NA & $\begin{array}{l}\text { ESP } 34.3 \\
\text { JBR } 93.5\end{array}$ \\
\hline $\begin{array}{l}\text { Springerville } \\
\text { Generating } \\
\text { Station, } \\
\text { M29 }\end{array}$ & $\begin{array}{l}846 \\
857 \\
891\end{array}$ & 865 & 24 & NA & $\begin{array}{c}106 \\
115 \\
P\end{array}$ & $\begin{array}{c}154 \\
122 \\
F\end{array}$ & NA & NA & NA & 576 & 37 & $\begin{array}{l}\text { FF } 98.0 \\
\text { SDA-FF } 90.5\end{array}$ \\
\hline
\end{tabular}


$\frac{1}{1}$

Table A-6. Total Cadmium

\begin{tabular}{|c|c|c|c|c|c|c|c|c|c|c|c|c|c|}
\hline \multirow{2}{*}{$\begin{array}{c}\text { Station/ } \\
\text { Plant } \\
\text { Name }\end{array}$} & \multirow{2}{*}{$\begin{array}{l}\text { Total } \\
\text { Stack } \\
\text { Conc.. } \\
\mu \mathrm{g} / \mathrm{Nm}^{3}\end{array}$} & \multirow{2}{*}{$\begin{array}{c}\text { Total } \\
\text { Mean } \\
\text { Conc.. } \\
\mu \mathrm{g} / \mathrm{Nm}^{3}\end{array}$} & \multirow[b]{2}{*}{$\begin{array}{l}\text { Standard } \\
\text { Deviation }\end{array}$} & \multirow{2}{*}{$\begin{array}{l}\text { Blank } \\
\text { Conc. } \\
\text { Range, } \\
\mu \mathrm{g} / \mathrm{Nm}^{3}\end{array}$} & \multicolumn{2}{|c|}{$\begin{array}{c}\text { M29 Internal Spike Recovery. } \\
\%\end{array}$} & \multicolumn{2}{|c|}{$\begin{array}{l}\text { M29 Audit Spike Recovery, } \\
\%\end{array}$} & \multirow[b]{2}{*}{$\begin{array}{c}\text { Above } \\
\text { DL }\end{array}$} & \multirow[b]{2}{*}{$\begin{array}{c}\text { Above } \\
\text { LLO }\end{array}$} & \multirow{2}{*}{$\begin{array}{l}\text { Emission } \\
\text { Factor, } \\
\mathrm{Ib} / 10^{12} \mathrm{Btu}\end{array}$} & \multirow{2}{*}{$\begin{array}{l}\text { Emission } \\
\text { Factor } \\
95 \% \mathrm{Cl}\end{array}$} & \multirow{2}{*}{$\begin{array}{c}\text { Control } \\
\text { Device } \\
\text { Removal, } \\
\%\end{array}$} \\
\hline & & & & & Filter & Peroxide & Filter & Peroxide & & & & & \\
\hline $\begin{array}{l}\text { Bailly } \\
\text { Station, } \\
\text { M29 }\end{array}$ & $\begin{array}{l}0.79 \\
0.38 \\
0.54\end{array}$ & 0.57 & 0.21 & $P$ & $\begin{array}{c}88.1 \\
88.1 \\
P\end{array}$ & $\begin{array}{c}83, \\
88.3 \\
P\end{array}$ & $\begin{array}{c}116 . \\
115 \\
P\end{array}$ & $\begin{array}{l}76, \\
77 \\
P\end{array}$ & NA & NA & 0.42 & 0.39 & $\begin{array}{l}\text { ESP\#7 } 93.78 \\
\text { ESP\#8 } 97.33 \\
\text { AFGD } 90.15\end{array}$ \\
\hline $\begin{array}{c}\text { Baldwin } \\
\text { Power Station, } \\
\text { M29 } \\
\text { Non-Sootblowing }\end{array}$ & $\begin{array}{l}4 \\
4 \\
4\end{array}$ & 4 & 0 & $P$ & NA & NA & $\begin{array}{c}90 \\
177 \\
P\end{array}$ & $\begin{array}{l}50, \\
95 \\
\mathrm{~F}\end{array}$ & $P$ & $P$ & 3.0 & 0.31 & ESP 95 \\
\hline $\begin{array}{c}\text { Baldwin } \\
\text { Power Station, } \\
\text { M29 } \\
\text { Sootblowing }\end{array}$ & $\begin{array}{l}5 \\
7 \\
6\end{array}$ & 6 & 1 & $P$ & NA & NA & $\begin{array}{c}90 . \\
177 \\
P\end{array}$ & $\begin{array}{c}50 \\
95 \\
F\end{array}$ & $P$ & $P$ & 4.5 & 1.9 & ESP 96 \\
\hline $\begin{array}{c}\text { Boswell } \\
\text { Energy Center. } \\
\text { M29 }\end{array}$ & $\begin{array}{l}N D<0.84 \\
N D<0.87 \\
N D<0.87\end{array}$ & $N D<0.86$ & 0.017 & $P$ & NA & NA & $\begin{array}{c}175 \\
263 \\
F\end{array}$ & $\begin{array}{c}7 t \\
111 \\
P\end{array}$ & $F$ & $F$ & $N D<0.65$ & 0.65 & $F F>94$ \\
\hline $\begin{array}{c}\text { Cardinal } \\
\text { Plant. } \\
\text { M29 } \\
\text { Non-Sootblowing }\end{array}$ & $\begin{array}{l}0.198 \\
3.19 \\
0.165 \\
0.859\end{array}$ & 1.103 & 1.427 & $F$ & $\begin{array}{c}74 \\
P\end{array}$ & $\begin{array}{c}71 \\
p\end{array}$ & $\begin{array}{c}80 \\
110 \\
P\end{array}$ & $\begin{array}{l}83 \\
P\end{array}$ & $P$ & $P$ & 0.85 & 1.8 & ESP 97,18 \\
\hline $\begin{array}{l}\text { Cardinal Plant, } \\
\text { M29 } \\
\text { Sootblowing }\end{array}$ & $\begin{array}{l}0.482 \\
0.460 \\
1.63\end{array}$ & 0.857 & 0.669 & $F$ & $\begin{array}{c}74 \\
P\end{array}$ & $\begin{array}{c}71 \\
P\end{array}$ & $\begin{array}{c}80 \\
110 \\
P\end{array}$ & $\begin{array}{c}83 \\
P\end{array}$ & $P$ & $\mathbf{P}$ & 0.66 & 1.3 & \\
\hline $\begin{array}{c}\text { Coal Creek } \\
\text { Station, } \\
\text { M29 } \\
\end{array}$ & $\begin{array}{l}N D<4.51 \\
N D<4.19 \\
N D<4.2 \\
\end{array}$ & ND $<4.31$ & 0.18 & $\begin{array}{c}N D<2.69 \\
N D<5.69 \\
P \\
\end{array}$ & $\begin{array}{c}90-98 \\
P\end{array}$ & $\begin{array}{c}90.6 \\
P\end{array}$ & $\begin{array}{l}76 . \\
70 \\
\mathrm{P} \\
\end{array}$ & $\begin{array}{c}0 \\
150 \\
F \\
\end{array}$ & $\mathrm{~F}$ & $\mathrm{~F}$ & $N D<3.2$ & 3.2 & $\begin{array}{c}\text { ESP\#1 } 87.2 \\
\text { Wet FGD } 46.2 \\
\text { Combined } \\
85.6\end{array}$ \\
\hline $\begin{array}{l}\text { Niles } \\
\text { Station, } \\
\text { M29 }\end{array}$ & $\begin{array}{c}\text { NO }<0.1 \\
\text { ND }<0.1 \\
0.24\end{array}$ & 0.11 & 0.11 & ND $<0.114$ & \multicolumn{2}{|c|}{$\begin{array}{c}76-147 \\
F\end{array}$} & $\begin{array}{c}55, \\
88 \\
F\end{array}$ & $\begin{array}{l}72, \\
77 \\
P\end{array}$ & $\mathrm{~F}$ & $\mathrm{~F}$ & 0.07 & 0.16 & ESP 97.11 \\
\hline $\begin{array}{l}\text { Niles/SNOX } \\
\text { Station, } \\
\text { M29. }\end{array}$ & $\begin{array}{c}0.230 \\
\text { ND }<0.138 \\
\text { ND }<0.162\end{array}$ & $\mathrm{ND}<0.162$ & 0.090 & $\begin{array}{c}\text { ND }<0.11 \\
P\end{array}$ & \multicolumn{2}{|c|}{$\underset{F}{76-147}$} & $\begin{array}{l}55, \\
88 \\
F\end{array}$ & $\begin{array}{l}72, \\
77 \\
\mathrm{~F}\end{array}$ & $F$ & $F$ & 0.092 & 0.16 & $\begin{array}{l}\text { FF } 99.04 \\
\text { SNOX } 97.90 \\
\end{array}$ \\
\hline $\begin{array}{l}\text { Plant } \\
\text { Yates, } \\
\text { M29 }\end{array}$ & $\begin{array}{c}\text { ND }<0.11^{x} \\
0.46 \\
0.79\end{array}$ & 0.62 & 0.23 & $\begin{array}{c}0.054 \\
P\end{array}$ & NA & $\begin{array}{c}114 \\
P\end{array}$ & $\begin{array}{c}95.9 \\
70.7 \\
P\end{array}$ & $\begin{array}{c}123.6 \\
113.8 \\
P\end{array}$ & $\mathbf{P}$ & $\mathrm{F}$ & 0.6 & 2.1 & $\begin{array}{l}\text { ESP } 95.1 \\
\text { JBR } 46.2 \\
\end{array}$ \\
\hline $\begin{array}{l}\text { Springerville } \\
\text { Generating } \\
\text { Station, } \\
\text { M29 }\end{array}$ & $\begin{array}{l}0.80^{*} \\
0.014 \\
0.06\end{array}$ & 0.037 & 0.032 & NA & $\begin{array}{c}72, \\
64 \\
F\end{array}$ & $\begin{array}{c}94 \\
96 \\
\mathrm{P}\end{array}$ & NA & NA & NA & NA & 0.026 & 0.053 & $\begin{array}{c}\text { FF } 99.99 \\
\text { SDA-FF } 99.99\end{array}$ \\
\hline
\end{tabular}

y - Value considered an outlier. Note: A detailed discussion of QA/OC criteria is presented in Section 4.2.1. 
Table A-7. Total Chlorine Species

\begin{tabular}{|c|c|c|c|c|c|c|c|c|c|c|c|}
\hline $\begin{array}{c}\text { Station/ } \\
\text { Plant } \\
\text { Name }\end{array}$ & $\begin{array}{c}\text { Total } \\
\text { Stack } \\
\text { Conc, } \\
\mu \mathrm{g} / \mathrm{Nm}^{3}\end{array}$ & $\begin{array}{l}\text { Total } \\
\text { Mean } \\
\text { Conc., } \\
\mu \mathrm{g} / \mathrm{Nm}^{3}\end{array}$ & $\begin{array}{l}\text { Standard } \\
\text { Deviation }\end{array}$ & $\begin{array}{c}\text { Blank } \\
\text { Conc. } \\
\text { Range. } \\
\mu \mathrm{g} / \mathrm{Nm}^{3}\end{array}$ & $\begin{array}{c}\text { Internal } \\
\text { Spike } \\
\text { Recovery. } \\
\%\end{array}$ & $\begin{array}{c}\text { Audit } \\
\text { Spike } \\
\text { Recovery, } \\
\%\end{array}$ & $\begin{array}{c}\text { Above } \\
\mathrm{DL}\end{array}$ & $\begin{array}{c}\text { Above } \\
\text { LLa }\end{array}$ & $\begin{array}{c}\text { Emission } \\
\text { Factor, } \\
\text { Ib } / 10^{12} \text { Btu }\end{array}$ & $\begin{array}{c}\text { Emission } \\
\text { Factor } \\
95 \% \mathrm{Cl}\end{array}$ & $\begin{array}{c}\text { Control } \\
\text { Device } \\
\text { Removal, } \\
\%\end{array}$ \\
\hline $\begin{array}{c}\text { Bailly } \\
\text { Station } \\
\text { HCl }\end{array}$ & $\begin{array}{l}1480 \\
1220 \\
1440\end{array}$ & 1380 & 140 & NA & $\begin{array}{l}99 \\
\mathrm{P}\end{array}$ & NA & NA & NA & 1020 & 260 & AFGD 99 \\
\hline $\begin{array}{c}\text { Baldwin } \\
\text { Power Station } \\
\text { Chloride }\end{array}$ & $\begin{array}{l}\text { ND }<5.89 \\
N D<11.3 \\
\text { ND }<12.5\end{array}$ & ND $<9.9$ & 3.5 & NA & NA & NA & NA & NA & ND $<7.3$ & 7.3 & $E S P>94$ \\
\hline $\begin{array}{c}\text { Baldwin } \\
\text { Power Station } \\
\text { Chlorine }\end{array}$ & $\begin{array}{l}4850 \\
9050 \\
4400\end{array}$ & 6100 & 2565 & NA & $p$ & NA & $p$ & $p$ & 4600 & 5000 & NA \\
\hline $\begin{array}{c}\text { Baldwin } \\
\text { Power Station } \\
\mathrm{HCl}\end{array}$ & $\begin{array}{l}108000 \\
103000 \\
105000\end{array}$ & 105000 & 2550 & NA & $P$ & NA & $P$ & $P$ & 78000 & 3600 & NA \\
\hline $\begin{array}{c}\text { Boswell } \\
\text { Energy Center } \\
\text { Chloride }\end{array}$ & $\begin{array}{l}N D<5.18 \\
N D<6.59 \\
N D<5.6\end{array}$ & $N D<5.79$ & 0.72 & NA & $P$ & $\mathbf{P}$ & NA & NA: & $N D<4.5$ & 4.5 & $F F>94$ \\
\hline $\begin{array}{c}\text { Boswell } \\
\text { Energy Center } \\
\text { Chlorine }\end{array}$ & $\begin{array}{c}112 \\
844 \\
1570\end{array}$ & 842 & 729 & NA & $p$ & NA & $P$ & $\mathrm{~F}$ & 640 & 1400 & $\mathrm{FF} 76$ \\
\hline $\begin{array}{c}\text { Boswell } \\
\text { Energy Center } \\
\mathrm{HCl}\end{array}$ & $\begin{array}{l}747 \\
1100 \\
1200\end{array}$ & 1020 & 312 & NA & $P$ & NA & $\mathrm{F}$ & $\mathrm{F}$ & 790 & 380 & FF 45 \\
\hline $\begin{array}{c}\text { Cardinal } \\
\text { Plant } \\
\text { Chlorine }\end{array}$ & $\begin{array}{l}1670 \\
2340\end{array}$ & 2005 & 473.8 & NA & $\mathrm{P}$ & NA & $p$ & $p$ & 1550 & 1200 & $\operatorname{ESP}(-)$ \\
\hline $\begin{array}{c}\text { Cardinal } \\
\text { Plant } \\
\mathrm{HCl}\end{array}$ & $\begin{array}{l}30600 \\
25900 \\
32500\end{array}$ & 29667 & 3398 & NA & $P$ & NA & $p$ & $p$ & 22900 & 12900 & ESP 24 \\
\hline $\begin{array}{l}\text { Coal Creek } \\
\text { Station } \\
\text { Chloride }\end{array}$ & $\begin{array}{c}8.83 \\
\mathrm{ND}<0.953 \\
4.72\end{array}$ & 4.7 & 4.2 & $\begin{array}{l}5.09 \\
4.32 \\
F\end{array}$ & $\begin{array}{c}109 \\
P\end{array}$ & NA & \multirow{2}{*}{$\mathbf{p}$} & \multirow{2}{*}{$\mathrm{P}$} & 3.5 & 7.6 & NA \\
\hline $\begin{array}{l}\text { Coal Creek } \\
\text { Station } \\
\text { HCI }\end{array}$ & $\begin{array}{l}1980 \\
1720 \\
1690\end{array}$ & 1800 & 160 & $\begin{array}{c}14.3 \\
460 \\
P\end{array}$ & NA & NA & & & 1340 & 285 & NA \\
\hline $\begin{array}{c}\text { Niles } \\
\text { Station } \\
\text { Chloride }\end{array}$ & $\begin{array}{l}14.1 \\
39.3 \\
40.2\end{array}$ & 31 & 15 & $\begin{array}{c}4.89 \\
P\end{array}$ & $\begin{array}{c}98, \\
125 \\
P\end{array}$ & NA & \multirow{2}{*}{$P$} & \multirow{2}{*}{$P$} & 19 & 21 & ESP 95 \\
\hline $\begin{array}{c}\text { Niles } \\
\text { Station } \\
\mathrm{HCl}\end{array}$ & $\begin{array}{l}221302 \\
218101 \\
218635 \\
\end{array}$ & 219346 & 1715 & $\begin{array}{c}26.9 \\
P\end{array}$ & NA & NA & & & 132000 & 2562 & NA \\
\hline $\begin{array}{c}\text { Niles/SNOXX } \\
\text { Station } \\
\text { Chloride }\end{array}$ & $\begin{array}{c}6.6 \\
\text { No }<16.7 \\
87.2\end{array}$ & 34 & 46 & $\begin{array}{c}16.1 \\
F\end{array}$ & $\begin{array}{c}98, \\
125 \\
P\end{array}$ & NA & \multirow{2}{*}{$P$} & \multirow{2}{*}{$\mathrm{F}$} & 25 & 82 & NA \\
\hline $\begin{array}{c}\text { Niles/SNOX } \\
\text { Station } \\
\text { HCI }\end{array}$ & $\begin{array}{c}109000 \\
137000 \\
93700\end{array}$ & 113000 & 22000 & $\begin{array}{c}10.4 \\
P\end{array}$ & NA & NA & & & .82400 & 41800 & NA \\
\hline $\begin{array}{c}\text { Plant } \\
\text { Yates } \\
\text { Chloride }\end{array}$ & $\begin{array}{c}345.2 \\
203.6 \\
93.4\end{array}$ & 214.1 & 126.2 & NA & $\begin{array}{c}95 \\
P\end{array}$ & NA & NA & NA & \multirow{2}{*}{742} & \multirow{2}{*}{647} & ESP 99.3 \\
\hline $\begin{array}{c}\text { Plant } \\
\text { Yates } \\
\text { HCI }\end{array}$ & $\begin{array}{l}294 \\
914 \\
411\end{array}$ & 540 & 329 & NA & $\begin{array}{c}100 \\
p\end{array}$ & NA & NA & NA & & & JBR 99.6 \\
\hline $\begin{array}{l}\text { Springerville } \\
\text { Generating } \\
\text { Station, } \\
\text { HCI }\end{array}$ & $\begin{array}{c}N D<240 \\
N D<250 \\
220\end{array}$ & $N D<155$ & 56.3 & NA & NA & NA & NA & NA & ND $<176$ & 176 & NA \\
\hline
\end{tabular}

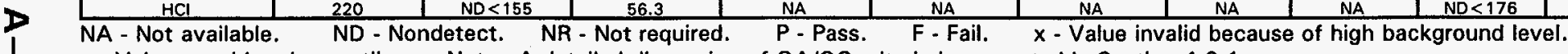

y $y$-Value considered an outlier. Note: A detailed discussion of QA/OC criteria is presented in Section 4.2.1. 


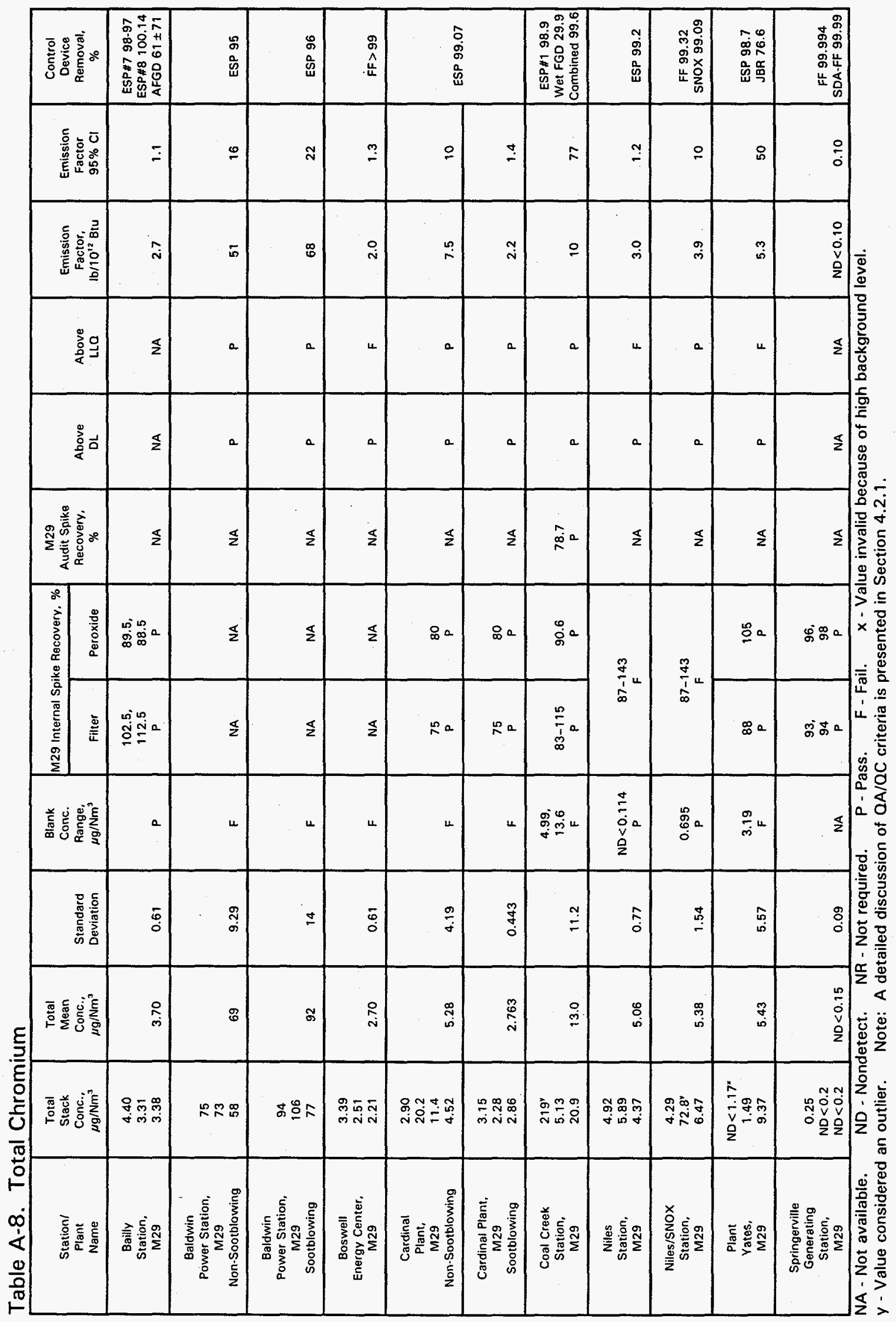


Table A-9. Total Cobalt

\begin{tabular}{|c|c|c|c|c|c|c|c|c|c|c|c|c|}
\hline \multirow{2}{*}{$\begin{array}{c}\text { Station/ } \\
\text { Plant } \\
\text { Name }\end{array}$} & \multirow{2}{*}{$\begin{array}{c}\text { Total } \\
\text { Stack } \\
\text { Conc.., } \\
\mu \mathrm{g} / \mathrm{Nm}^{3}\end{array}$} & \multirow{2}{*}{$\begin{array}{c}\text { Total } \\
\text { Mean } \\
\text { Conc., } \\
\mu \mathrm{g} / \mathrm{Nm}^{3}\end{array}$} & \multirow[b]{2}{*}{$\begin{array}{l}\text { Standard } \\
\text { Deviation }\end{array}$} & \multirow{2}{*}{$\begin{array}{c}\text { Blank } \\
\text { Conc. } \\
\text { Range. } \\
\mu \mathrm{g} / \mathrm{Nm}^{3}\end{array}$} & \multicolumn{2}{|c|}{ M29 Internal Spike Recovery, \% } & \multirow{2}{*}{$\begin{array}{c}\text { M29 } \\
\text { Audit Spike } \\
\text { Recovery. } \\
\%\end{array}$} & \multirow[b]{2}{*}{$\begin{array}{c}\text { Above } \\
\text { DL }\end{array}$} & \multirow[b]{2}{*}{$\begin{array}{c}\text { Above } \\
\text { LLo }\end{array}$} & \multirow{2}{*}{$\begin{array}{l}\text { Emission } \\
\text { Factor, } \\
\mathrm{Ib} / 10^{12} \text { Btu }\end{array}$} & \multirow{2}{*}{$\begin{array}{l}\text { Emission } \\
\text { Factor } \\
95 \% \mathrm{Cl}\end{array}$} & \multirow{2}{*}{$\begin{array}{c}\text { Control } \\
\text { Device } \\
\text { Removal, } \\
\%\end{array}$} \\
\hline & & & & & Filter & Peroxide & & & & & & \\
\hline $\begin{array}{l}\text { Bailly } \\
\text { Station, } \\
\text { M29 }\end{array}$ & $\begin{array}{l}0.16 \\
0.09 \\
0.14\end{array}$ & 0.13 & 0.04 & $\mathrm{~F}$ & $\begin{array}{c}104.5 \\
103 \\
P\end{array}$ & $\begin{array}{c}94.2 \\
90.2 \\
P\end{array}$ & NA & NA & NA & ND $<0.07$ & 0.07 & $\begin{array}{c}\text { ESP \#7 } 98.29 \\
\text { ESP \#8 } 99.62 \\
\text { AFGD } 79.3\end{array}$ \\
\hline $\begin{array}{c}\text { Baldwin } \\
\text { Power Station, } \\
\text { M29 } \\
\text { Non-Sootblowing }\end{array}$ & $\begin{array}{c}9 \\
10 \\
9\end{array}$ & 9 & 0.6 & $\mathbf{F}$ & NA & NA & NA & $\mathbf{P}$ & $\mathbf{P}$ & 6.8 & 0.86 & ESP 95 \\
\hline $\begin{array}{c}\text { Baldwin } \\
\text { Power Station, } \\
\text { M29 } \\
\text { Sootblowing }\end{array}$ & $\begin{array}{l}10 \\
18 \\
13\end{array}$ & 14 & 4 & $\mathrm{~F}$ & NA & NA & NA & $\mathbf{P}$ & $\mathbf{p}$ & 10 & 7.2 & ESP 96 \\
\hline $\begin{array}{c}\text { Boswell } \\
\text { Energy Center, } \\
\text { M29 }\end{array}$ & $\begin{array}{l}1.11 \\
0.64 \\
1.04\end{array}$ & 0.93 & 0.25 & $\mathrm{~F}$ & NA & NA & NA & $\mathbf{P}$ & $\mathrm{F}$ & 0.70 & 0.51 & $F F>99$ \\
\hline $\begin{array}{c}\text { Cardinal } \\
\text { Plant, } \\
\text { M29 } \\
\text { Non-Sootblowing }\end{array}$ & $\begin{array}{l}0.68 \\
0.925 \\
0.863 \\
0.789\end{array}$ & 0.814 & 0.105 & $\mathrm{~F}$ & $\begin{array}{c}72 \\
P\end{array}$ & $\begin{array}{c}76 \\
P\end{array}$ & NA & $\mathbf{P}$ & $\mathbf{F}$ & 0.63 & 0.28 & \\
\hline $\begin{array}{l}\text { Cardinal } \\
\text { Plant, } \\
\text { M29 } \\
\text { Sootblowing }\end{array}$ & $\begin{array}{l}0.375 \\
0.303 \\
0.692\end{array}$ & 0.457 & 0.207 & $\mathrm{~F}$ & $\begin{array}{c}72 \\
P\end{array}$ & $\begin{array}{c}76 \\
P\end{array}$ & NA & $\mathbf{P}$ & $F$ & 0.30 & 0.53 & cor 50.02 \\
\hline $\begin{array}{l}\text { Coal Creek } \\
\text { Station, } \\
\text { M29 }\end{array}$ & $\begin{array}{c}3.96 \\
N D<2.21 \\
N D<2.21\end{array}$ & ND $<2.21$ & 1.65 & $\begin{array}{c}N D<1.71 \\
N D<4.67 \\
F\end{array}$ & $\underset{P}{96-112}$ & $\begin{array}{l}104 \\
P\end{array}$ & NA & $\mathbf{F}$ & $\mathbf{F}$ & $N D<1.5$ & 1.5 & $\begin{array}{c}\text { ESP\#1 } 99.6 \\
\text { Wet FGD } 44.7 \\
\text { Combined } 99.2\end{array}$ \\
\hline $\begin{array}{l}\text { Niles } \\
\text { Station, } \\
\text { M29 }\end{array}$ & $\begin{array}{l}N D<0.20 \\
\text { ND }<0.19 \\
\text { ND }<0.20\end{array}$ & $\mathrm{ND}<0.20$ & 0.01 & $\begin{array}{c}N D<0.228 \\
P\end{array}$ & \multicolumn{2}{|c|}{$\begin{array}{c}81-130 \\
F\end{array}$} & NA & $\mathbf{F}$ & $\mathrm{F}$ & ND $<0.12$ & 0.12 & ESP 99.95 \\
\hline $\begin{array}{l}\text { Niles/SNOX } \\
\text { Station, } \\
\text { M29 }\end{array}$ & $\begin{array}{c}N D<0.275 \\
N D<0.324 \\
1.14^{\gamma}\end{array}$ & ND $<0.300$ & 0.035 & $\begin{array}{c}N D<0.219 \\
P\end{array}$ & \multicolumn{2}{|c|}{$\begin{array}{c}81-130 \\
F\end{array}$} & NA & $\mathrm{F}$ & $\mathrm{F}$ & ND $<0.22$ & 0.22 & $\begin{array}{c}\text { FF } 99.96 \\
\text { SNOX } 99.93\end{array}$ \\
\hline $\begin{array}{l}\text { Plant } \\
\text { Yates, } \\
\text { M29 }\end{array}$ & $\begin{array}{l}1.38^{*} \\
0.42 \\
1.05\end{array}$ & 0.74 & 0.44 & $\begin{array}{l}1.01 \\
F\end{array}$ & $\begin{array}{c}91 \\
\mathrm{p}\end{array}$ & $\begin{array}{c}102 \\
P\end{array}$ & NA & $\mathrm{F}$ & $\mathbf{F}$ & 0.7 & 0.8 & $\begin{array}{l}\text { ESP } 98.2 \\
\text { JBR } 85.3\end{array}$ \\
\hline $\begin{array}{l}\text { Springerville } \\
\text { Generating } \\
\text { Station, } \\
\text { M29 }\end{array}$ & $\begin{array}{l}N D<0.4 \\
N D<0.4 \\
N D<0.4\end{array}$ & $N D<0.4$ & NA & NA & $\begin{array}{l}91, \\
96 \\
P\end{array}$ & $\begin{array}{l}96 . \\
96 \\
P\end{array}$ & NA & NA & NA & ND $<0.3$ & 0.3 & $\begin{array}{c}F F>99.96 \\
\text { SDA-FF }>99.91\end{array}$ \\
\hline
\end{tabular}

NA - Not available. ND - Nondetect. NR - Not required P - Pass, F - Fail $x$ - Value invalid because of high background level.

$y$ - Value considered an outlier. Note: A detailed discussion of QA/OC criteria is presented in Section 4.2.1. 
NA - Not available ND - Nondetect. NR - Not required P - Pass. F - Fail. $x$ - Value invalid because of high background level.

$y$-Value considered an outlier. Note: A detailed discussion of QA/QC criteria is presented in Section 4.2.1. 
Table A-11. Total Cyanide

\begin{tabular}{|c|c|c|c|c|c|c|c|c|c|c|c|}
\hline $\begin{array}{c}\text { Station/ } \\
\text { Plant } \\
\text { Name }\end{array}$ & $\begin{array}{l}\text { Total } \\
\text { Stack } \\
\text { Conc., } \\
\mu \mathrm{g} / \mathrm{Nm}^{3}\end{array}$ & $\begin{array}{c}\text { Total } \\
\text { Mean } \\
\text { Conc.., } \\
\mu \mathrm{g} / \mathrm{Nm}^{3}\end{array}$ & $\begin{array}{l}\text { Standard } \\
\text { Deviation }\end{array}$ & $\begin{array}{l}\text { Blank } \\
\text { Conc. } \\
\text { Aange, } \\
\mu \mathrm{g} / \mathrm{Nm}^{3}\end{array}$ & $\begin{array}{c}\text { Internal } \\
\text { Spike } \\
\text { Recovery. } \\
\%\end{array}$ & $\begin{array}{c}\text { Audit } \\
\text { Spike } \\
\text { Recovery. } \\
\%\end{array}$ & $\begin{array}{c}\text { Above } \\
\text { DL }\end{array}$ & $\begin{array}{c}\text { Above } \\
\text { LLO }\end{array}$ & $\begin{array}{l}\text { Emission } \\
\text { Factor, } \\
\mathrm{Ib} / 10^{12} \mathrm{Btu}\end{array}$ & $\begin{array}{c}\text { Emission } \\
\text { Factor } \\
95 \% \mathrm{Cl}\end{array}$ & $\begin{array}{c}\text { Control } \\
\text { Device } \\
\text { Removal, } \\
\%\end{array}$ \\
\hline $\begin{array}{l}\text { Bailly } \\
\text { Station } \\
\text { HCN }\end{array}$ & 15.6 & NA & NA & NA & $\begin{array}{c}97.1 \\
P\end{array}$ & NA & NA & NA & NA & NA & NA \\
\hline $\begin{array}{c}\text { Baldwin } \\
\text { Power Station, } \\
\text { Particulate CN- }\end{array}$ & $\begin{array}{l}N D<1.47 \\
N D<1.41 \\
\text { ND }<1.56\end{array}$ & $N D<1.48$ & 0.76 & NA & NA & NA & $F$ & $\mathbf{F}$ & ND $<1.1$ & 1.1 & ESP $>41$ \\
\hline $\begin{array}{l}\text { Baldwin } \\
\text { Power Station, } \\
\text { HCN }\end{array}$ & $\begin{array}{l}\text { ND }<2.97 \\
\text { ND }<3.13 \\
\text { ND }<2.97\end{array}$ & $N D<3.02$ & 0.09 & NA & $P$ & NA & $F$ & $\mathrm{~F}$ & ND $<2.2$ & 2.2 & $E S P>30$ \\
\hline $\begin{array}{c}\text { Boswell } \\
\text { Energy Center, } \\
\text { Particulate CN- }\end{array}$ & $\begin{array}{l}N D<1.3 \\
N D<1.65 \\
N D<1.4\end{array}$ & $\mathrm{ND}<1.45$ & 0.18 & NA & $\mathbf{P}$ & NA & $F$ & $F$ & $N D<1.1$ & 1.1 & $F F>51$ \\
\hline $\begin{array}{c}\text { Boswell } \\
\text { Energy Center. } \\
\text { HCN }\end{array}$ & $\begin{array}{l}3.11 \\
7.23 \\
3.95\end{array}$ & 4.76 & 2.17 & NA & $P$ & NA & $P$ & $F$ & 3.7 & 3.8 & FF 35 \\
\hline $\begin{array}{l}\text { Cardinal } \\
\text { Plant, } \\
\text { HCN }\end{array}$ & $\begin{array}{l}0.817 \\
0.714\end{array}$ & 0.766 & 0.073 & NA & $P$ & NA & NA & NA & 0.59 & 0.050 & NA \\
\hline $\begin{array}{c}\text { Coal Creek } \\
\text { Station, } \\
\text { HCN }\end{array}$ & $\begin{array}{l}56.1 \\
48.4 \\
99.0\end{array}$ & 68 & 27 & $\begin{array}{c}3.30 \\
65.5 \\
F\end{array}$ & $\begin{array}{c}93, \\
94 \\
\mathrm{P}\end{array}$ & NA & $F$ & $\mathbf{F}$ & 51 & 51 & $N A$ \\
\hline $\begin{array}{l}\text { Niles } \\
\text { Station, } \\
\text { HCN }\end{array}$ & $\begin{array}{l}115 \\
280 \\
513 \\
\end{array}$ & 303 & 200 & $\begin{array}{c}3.87 \\
P\end{array}$ & $\begin{array}{c}85-105 \\
P\end{array}$ & NA & $P$ & $F$ & 180 & 288 & NA \\
\hline $\begin{array}{l}\text { Niles/SNOX, } \\
\text { Station } \\
\text { HCN }\end{array}$ & $\begin{array}{l}181 \\
198 \\
269 \\
\end{array}$ & 216 & 47 & $\begin{array}{c}2.49 \\
P\end{array}$ & $\begin{array}{c}85-105 \\
P\end{array}$ & NA & $P$ & $F$ & 157 & 82 & NA \\
\hline $\begin{array}{c}\text { Plant } \\
\text { Yates, } \\
\text { HCN }\end{array}$ & $\begin{array}{r}4.87 \\
8.65 \\
71.99 \\
\end{array}$ & 28.47 & 37.73 & NA & $\begin{array}{c}50 \\
F\end{array}$ & NA & NA & NA & NA & NA & NA \\
\hline $\begin{array}{l}\text { Springerville } \\
\text { Generating } \\
\text { Station. } \\
\text { HCN }\end{array}$ & $\begin{array}{l}40 \\
5.6 \\
6.0\end{array}$ & 17.2 & 19.7 & NA & NA & NA & NA & $\mathrm{NA}$ & 12 & 33 & NA \\
\hline
\end{tabular}

NA - Not available. ND - Nondetect. NR - Not required. P - Pass. F - Fail. $x$ - Value invalid because of high background level.

$y$-Value considered an outlier. Note: A detailed discussion of QA/OC criteria is presented in Section 4.2.1. 
Table A-12. Total Fluorine Species

\begin{tabular}{|c|c|c|c|c|c|c|c|c|c|c|c|}
\hline $\begin{array}{c}\text { Station/ } \\
\text { Plant } \\
\text { Name }\end{array}$ & $\begin{array}{l}\text { Total } \\
\text { Stack } \\
\text { Conc.., } \\
\mu \mathrm{g} / \mathrm{Nm}^{3}\end{array}$ & $\begin{array}{c}\text { Total } \\
\text { Mean } \\
\text { Conc.. } \\
\mu \mathrm{g} / \mathrm{Nm}^{3}\end{array}$ & $\begin{array}{l}\text { Standard } \\
\text { Deviation }\end{array}$ & $\begin{array}{l}\text { Blank } \\
\text { Conc. } \\
\text { Range, } \\
\mu \mathrm{g} / \mathrm{Nm}^{3}\end{array}$ & $\begin{array}{c}\text { Internal } \\
\text { Spike } \\
\text { Recovery. } \\
\%\end{array}$ & $\begin{array}{c}\text { Audit } \\
\text { Spike } \\
\text { Recovery. } \\
\%\end{array}$ & $\begin{array}{c}\text { Above } \\
\text { DL }\end{array}$ & $\begin{array}{c}\text { Above } \\
\text { LLa }\end{array}$ & $\begin{array}{c}\text { Emission } \\
\text { Factor, } \\
\text { Ib } / 10^{12} \text { Btu }\end{array}$ & $\begin{array}{c}\text { Emission } \\
\text { Factor } \\
95 \% \mathrm{Cl}\end{array}$ & $\begin{array}{c}\text { Control } \\
\text { Device } \\
\text { Removal, } \\
\%\end{array}$ \\
\hline $\begin{array}{c}\text { Bailly } \\
\text { Station } \\
\text { HF }\end{array}$ & $\begin{array}{l}N D<487 \\
N D<556 \\
N D<444\end{array}$ & $\mathrm{ND}<496$ & 56.5 & NA & NA & $\mathrm{NA}$ & NA & NA & $N D<420$ & 420 & AFGD 96 \\
\hline $\begin{array}{l}\text { Baldwin Power } \\
\text { Station, } \\
\text { Fluoride }\end{array}$ & $\begin{array}{c}N D<40 \\
N D<43.1 \\
N D<43.8\end{array}$ & NO $<42.3$ & 2.0 & NA & NA & NA & $F$ & $F$ & $N D<31$ & 31 & $E S P>55$ \\
\hline $\begin{array}{l}\text { Baldwin Power } \\
\text { Station } \\
\text { HF }\end{array}$ & $\begin{array}{c}15800 \\
14700 \\
9590 \\
\end{array}$ & 13400 & 3300 & NA & $\mathbf{p}$ & NA & $P$ & $P$ & 9900 & 6400 & NA \\
\hline $\begin{array}{l}\text { Baldwin Power } \\
\text { Station, } \\
\text { Fluorine } \\
\end{array}$ & $\begin{array}{l}\text { ND }<141 \\
N D<588 \\
N D<140 \\
\end{array}$ & $\mathrm{ND}<290$ & 258 & NA & $p$ & NA & $F$ & $F$ & $\mathrm{ND}<220$ & 220 & NA \\
\hline $\begin{array}{l}\text { Boswell Energy } \\
\text { Center, } \\
\text { Fluoride. }\end{array}$ & $\begin{array}{l}31.1 \\
13.2 \\
11.2 \\
\end{array}$ & 14.4 & 12.1 & NA & NA & NA & NA & NA & 12 & 24 & FF 88 \\
\hline $\begin{array}{l}\text { Boswell Energy } \\
\text { Center, } \\
\text { Fluorine }\end{array}$ & $\begin{array}{l}456 \\
1270 \\
1480\end{array}$ & 1070 & 541 & NA & $P$ & NA & $p$ & $p$ & 810 & 970 & FF 62 \\
\hline $\begin{array}{c}\text { Boswell Energy } \\
\text { Center, } \\
\text { HF }\end{array}$ & $\begin{array}{l}3260 \\
3270 \\
3030 \\
\end{array}$ & 3190 & 1360 & $\mathrm{NA}$ & NA & NA & $p$ & $F$ & 2500 & 500 & NA \\
\hline $\begin{array}{c}\text { Cardinal } \\
\text { Plant, } \\
\text { Fluorine } \\
\end{array}$ & $\begin{array}{l}15000 \\
13300 \\
\end{array}$ & 14100 & NA & NA & $\mathbf{P}$ & NA & NA & NA & 10900 & 8300 & NA \\
\hline $\begin{array}{l}\text { Cardinal } \\
\text { Plant, } \\
\text { HF }\end{array}$ & $\begin{array}{l}2540 \\
2210 \\
2500\end{array}$ & 2417 & 180 & NA & $p$ & NA & $P$ & $p$ & 1870 & 968.7 & $\operatorname{ESP}(-)$ \\
\hline $\begin{array}{l}\text { Coal Creek } \\
\text { Station, } \\
\text { Fluoride } \\
\end{array}$ & $\begin{array}{l}173 \\
233 \\
183 \\
\end{array}$ & 196 & 32 & $\begin{array}{c}\text { ND }<0.179 \\
0.963 \\
P\end{array}$ & $\begin{array}{c}94 \\
\mathrm{P} \\
\end{array}$ & NA & \multirow{2}{*}{$\mathrm{P}$} & \multirow{2}{*}{$\mathbf{P}$} & 146 & 64 & NA \\
\hline $\begin{array}{l}\text { Coal Creek } \\
\text { Station. } \\
\text { HF }\end{array}$ & $\begin{array}{l}7330 \\
5280 \\
3410\end{array}$ & 5340 & 1960 & $\begin{array}{c}0.507 \\
26.8 \\
P\end{array}$ & NA & NA & & & 3980 & 3560 & NA \\
\hline $\begin{array}{c}\text { Niles } \\
\text { Station, } \\
\text { Fluoride }\end{array}$ & $\begin{array}{l}8.27 \\
15.9 \\
32.1\end{array}$ & 18.8 & 12.2 & $\begin{array}{c}0.550 \\
P\end{array}$ & $P$ & $\mathbf{P}$ & \multirow{2}{*}{$P$} & \multirow{2}{*}{$F$} & 11 & 18 & ESP 95.1 \\
\hline $\begin{array}{l}\text { Niles } \\
\text { Station, } \\
\text { HF }\end{array}$ & $\begin{array}{l}12767 \\
15731 \\
16095\end{array}$ & 14864 & 1826 & $\stackrel{4.83}{P}$ & NA & NA & & & 8921 & 2455 & NA \\
\hline $\begin{array}{l}\text { Niles/SNOX } \\
\text { Station, } \\
\text { Fluoride }\end{array}$ & $\begin{array}{l}610 \\
5.80 \\
28.6\end{array}$ & 215 & 343 & $\stackrel{1.24}{P}$ & $p$ & NA & \multirow{2}{*}{$P$} & \multirow{2}{*}{$P$} & 157 & 621 & NA \\
\hline $\begin{array}{l}\text { Niles/SNOX } \\
\text { Station, } \\
\text { HF }\end{array}$ & $\begin{array}{c}9210 \\
10100 \\
8040 \\
\end{array}$ & 9120 & 1030 & $\begin{array}{c}13.5 \\
P \\
\end{array}$ & $\mathrm{NA}$ & $\mathrm{NA}$ & & & 6630 & 2110 & NA \\
\hline $\begin{array}{c}\text { Plant } \\
\text { Yates, } \\
\text { Fluoride }\end{array}$ & $\begin{array}{l}0.057 \\
0.063 \\
0.032\end{array}$ & 0.051 & 0.041 & NA & $\begin{array}{c}70 \\
\mathrm{P}\end{array}$ & NA & NA & NA & \multirow{2}{*}{122} & \multirow{2}{*}{67} & ESP 91 \\
\hline $\begin{array}{c}\text { Plant } \\
\text { Yates, } \\
\text { HF }\end{array}$ & $\begin{array}{c}126 \\
96 \\
150\end{array}$ & 124 & 27 & NA & $\begin{array}{c}107 \\
P\end{array}$ & NA & NA & NA & & & JBR 98.4 \\
\hline $\begin{array}{c}\text { Springerville } \\
\text { Generating } \\
\text { Station. } \\
\text { HF }\end{array}$ & $\begin{array}{l}N D<125 \\
N D<130 \\
N D<110\end{array}$ & NO $<121$ & 10.4 & NA & NA & NA & NA & NA & ND $<92$ & 92 & NA \\
\hline
\end{tabular}


Table A-13. Total Lead

\begin{tabular}{|c|c|c|c|c|c|c|c|c|c|c|c|c|c|}
\hline \multirow{2}{*}{$\begin{array}{c}\text { Station/ } \\
\text { Plant } \\
\text { Name }\end{array}$} & \multirow{2}{*}{$\begin{array}{c}\text { Total } \\
\text { Stack } \\
\text { Conc., } \\
\mu \mathrm{g} / \mathrm{Nm}^{3}\end{array}$} & \multirow{2}{*}{$\begin{array}{l}\text { Total } \\
\text { Mean } \\
\text { Conc., } \\
\mu \mathrm{g} / \mathrm{Nm}^{3}\end{array}$} & \multirow[b]{2}{*}{$\begin{array}{l}\text { Standard } \\
\text { Deviation }\end{array}$} & \multirow{2}{*}{$\begin{array}{c}\text { Blank } \\
\text { Conc. } \\
\text { Range, } \\
\mu \mathrm{g} / \mathrm{Nm}^{3}\end{array}$} & \multicolumn{2}{|c|}{$\begin{array}{l}\text { M29 Internal Spike } \\
\text { Recovery, \% }\end{array}$} & \multicolumn{2}{|c|}{$\begin{array}{l}\text { M29 Audit Spike } \\
\text { Recovery, \% }\end{array}$} & \multirow[b]{2}{*}{$\begin{array}{c}\text { Above } \\
\text { DL }\end{array}$} & \multirow[b]{2}{*}{$\begin{array}{l}\text { Above } \\
\text { LLo }\end{array}$} & \multirow{2}{*}{$\begin{array}{c}\text { Emission } \\
\text { Factor, } \\
\text { ib/10 }\end{array}$} & \multirow{2}{*}{$\begin{array}{l}\text { Emission } \\
\text { Factor } \\
95 \% \mathrm{Cl}\end{array}$} & \multirow{2}{*}{$\begin{array}{c}\text { Control } \\
\text { Device } \\
\text { Removal, } \\
\%\end{array}$} \\
\hline & & & & & Filter & Peroxide & Filter & Peroxide & & & & & \\
\hline $\begin{array}{l}\text { Bailly } \\
\text { Station, } \\
\text { M29 }\end{array}$ & $\begin{array}{l}3.52 \\
1.65 \\
1.34\end{array}$ & 2.13 & 1.21 & $\mathrm{~F}$ & $\begin{array}{l}93.6 \\
109.1 \\
P\end{array}$ & $\begin{array}{c}84 \\
91.1 \\
p\end{array}$ & $\begin{array}{c}120 \\
120 \\
P\end{array}$ & $\begin{array}{l}76, \\
90 \\
P\end{array}$ & NA & NA & 1.6 & 2.2 & $\begin{array}{l}\text { ESP\#7 } 98.34 \\
\text { ESP\#8 } 99.68 \\
\text { AFGD } 82.35\end{array}$ \\
\hline $\begin{array}{c}\text { Baldwin } \\
\text { Power Station, } \\
\text { M29 } \\
\text { Non-Sootblowing }\end{array}$ & $\begin{array}{l}41 \\
35 \\
41\end{array}$ & 39 & 3 & $\mathbf{F}$ & NA & NA & $\begin{array}{l}109, \\
<75 \mathrm{P}\end{array}$ & $\begin{array}{c}1290, \\
1212 \\
F\end{array}$ & $\mathbf{P}$ & $P$ & 29 & 6.5 & ESP 94 \\
\hline $\begin{array}{c}\text { Baldwin } \\
\text { Power Station, } \\
\text { M29 } \\
\text { Sootblowing }\end{array}$ & $\begin{array}{l}61 \\
66 \\
65\end{array}$ & 64 & 3 & $\mathrm{~F}$ & NA & NA & $\begin{array}{l}109, \\
<75 P\end{array}$ & $\begin{array}{c}1290 \\
1212 \\
F\end{array}$ & $\mathrm{P}$ & $\mathrm{P}$ & 48 & 8.4 & ESP 95 \\
\hline $\begin{array}{l}\text { Boswell } \\
\text { Energy Center, } \\
\text { M29 }\end{array}$ & $\begin{array}{c}3.55 \\
\text { ND }<2.17 \\
5.06\end{array}$ & 3.23 & 2.01 & $\mathbf{P}$ & $\mathrm{NA}$ & NA & $\begin{array}{c}219 \\
316 \\
F\end{array}$ & $\begin{array}{c}72 \\
185 \\
F\end{array}$ & $P$ & $F$ & 2.4 & 2.7 & FF 99 \\
\hline $\begin{array}{c}\text { Cardinal } \\
\text { Plant, } \\
\text { M29 } \\
\text { Non-Sootblowing }\end{array}$ & $\begin{array}{l}6.40 \\
5.05 \\
3.89 \\
4.49\end{array}$ & 4.96 & 1.07 & $\mathbf{F}$ & $\begin{array}{c}64 \\
F\end{array}$ & $\stackrel{21}{F}$ & $\begin{array}{c}73 \\
120 \\
P\end{array}$ & $\begin{array}{c}75 \\
P\end{array}$ & $\mathbf{P}$ & $\mathbf{P}$ & 3.8 & 2.7 & ESP 99,43 \\
\hline $\begin{array}{l}\text { Cardinal Plant, } \\
\text { M29 } \\
\text { Sootblowing }\end{array}$ & $\begin{array}{l}3.38 \\
3.04 \\
7.48\end{array}$ & 4.63 & 2.47 & $\mathbf{F}$ & $\begin{array}{c}64 \\
F\end{array}$ & $\begin{array}{l}21 \\
F\end{array}$ & $\begin{array}{c}73 \\
120 \\
P\end{array}$ & $\begin{array}{c}75 \\
P\end{array}$ & $P$ & $\mathbf{P}$ & 3.6 & 5.4 & \\
\hline $\begin{array}{l}\text { Coal Creek } \\
\text { Station, } \\
\text { M29 }\end{array}$ & $\begin{array}{l}1.06 \\
0.861 \\
0.849\end{array}$ & 0.923 & 0.119 & $\begin{array}{c}N D<3.16 \\
N D<94.7 \\
F\end{array}$ & $\begin{array}{c}100 \\
109 \\
P\end{array}$ & $\begin{array}{c}94 \\
103 \\
P\end{array}$ & $\begin{array}{l}3 \\
80 \\
F\end{array}$ & $\begin{array}{l}20, \\
88 \\
F\end{array}$ & $P$ & $\mathbf{P}$ & 0.69 & 0.21 & $\begin{array}{c}\text { ESPH1 } 99.8 \\
\text { Wet FGD 26.7 } \\
\text { Combined } 99.8\end{array}$ \\
\hline $\begin{array}{l}\text { Niles } \\
\text { Station, } \\
\text { M29 }\end{array}$ & $\begin{array}{l}2.62 \\
1.89 \\
3.47\end{array}$ & 2.66 & 0.79 & $\begin{array}{c}2.64 \\
F\end{array}$ & \multicolumn{2}{|c|}{$\begin{array}{c}85-109 \\
P\end{array}$} & $\begin{array}{l}87 \\
91 \\
P\end{array}$ & $\begin{array}{l}79 . \\
75 \\
P\end{array}$ & $\mathrm{P}$ & $P$ & 1.6 & 1.2 & ESP 99.72 \\
\hline $\begin{array}{l}\text { Niles/SNOX } \\
\text { Station, } \\
\text { M29 }\end{array}$ & $\begin{array}{c}N D<0.637 \\
N D<0.683 \\
1.53\end{array}$ & 0.730 & 0.693 & $\begin{array}{c}\mathrm{ND}<0.575 \\
\mathrm{P}\end{array}$ & \multicolumn{2}{|c|}{$\underset{p}{85-109}$} & $\begin{array}{c}87 . \\
91 \\
P\end{array}$ & $\begin{array}{c}79 . \\
75 \\
P\end{array}$ & $\mathrm{~F}$ & $\mathrm{~F}$ & 0.53 & 1.2 & $\begin{array}{l}\text { FF } 99.95 \\
\text { SNOX } 99.91\end{array}$ \\
\hline $\begin{array}{l}\text { Plant } \\
\text { Yates, } \\
\text { M29 }\end{array}$ & $\begin{array}{c}\text { ND }<0.20^{x} \\
0.67 \\
0.65\end{array}$ & 0.61 & 0.06 & $\begin{array}{l}1.08 \\
F\end{array}$ & NA & $\begin{array}{l}84 \\
P\end{array}$ & $\begin{array}{c}102 \\
101.2 \\
P\end{array}$ & $\begin{array}{l}98.9 \\
105.9 \\
P\end{array}$ & $\mathbf{P}$ & $\mathbf{F}$ & 0.6 & 0.6 & $\begin{array}{l}\text { ESP } 97.4 \\
\text { JBR } 96.7\end{array}$ \\
\hline $\begin{array}{l}\text { Springerville } \\
\text { Generating } \\
\text { Station, } \\
\text { M29 }\end{array}$ & $\begin{array}{c}1.0 \\
0.88 \\
1.2\end{array}$ & 1.0 & 0.3 & NA & $\begin{array}{c}92, \\
89 \\
P\end{array}$ & $\begin{array}{l}99, \\
99 \\
\mathrm{P}\end{array}$ & $\begin{array}{c}80.7 \\
79.5 \\
P\end{array}$ & $\begin{array}{c}86.7 \\
113.3 \\
P\end{array}$ & NA & NA & 0.67 & 0.51 & $\begin{array}{c}\text { FF } 99.7 \\
\text { SDA.FF } 99.4\end{array}$ \\
\hline
\end{tabular}

NA - Not available. ND - Nondetect. NR - Not required. P-Pass. F - Fail. $x$ - Value invalid because of high background level. 
Table A-14. Total Mercury

\begin{tabular}{|c|c|c|c|c|c|c|c|c|c|c|c|c|c|c|c|}
\hline \multirow{2}{*}{$\begin{array}{c}\text { Station/ } \\
\text { Plant } \\
\text { Name }\end{array}$} & \multirow{2}{*}{$\begin{array}{l}\text { Total Hg } \\
\text { Stack } \\
\text { Conc.. } \\
\mu g / \mathrm{Nm}^{3} \\
\end{array}$} & \multirow{2}{*}{$\begin{array}{l}\text { Total } \mathrm{Hg} \\
\text { Mean } \\
\text { Conc.. } \\
\mu \mathrm{g} / \mathrm{Nm}^{3} \\
\end{array}$} & \multirow{2}{*}{$\begin{array}{l}\text { Standard } \\
\text { Deviation }\end{array}$} & \multirow{2}{*}{$\begin{array}{l}\text { Blank Conc. } \\
\text { Range } \\
\mu \mathrm{g} / \mathrm{Nm}^{3}\end{array}$} & \multicolumn{3}{|c|}{ M29 Internal Spike Recovery, \% } & \multicolumn{3}{|c|}{ M29 Audit Spike Recovery, \% } & \multirow{2}{*}{$\begin{array}{c}\text { Above } \\
\text { DL }\end{array}$} & \multirow{2}{*}{$\begin{array}{l}\text { Above } \\
\text { LLO }\end{array}$} & \multirow{2}{*}{$\begin{array}{c}\text { Emission } \\
\text { Factor, } \\
1 \mathrm{~b} / 10^{12} \mathrm{Btu}\end{array}$} & \multirow{2}{*}{$\begin{array}{l}\text { Emission } \\
\text { Factor } \\
95 \% \mathrm{Cl} \\
\end{array}$} & \multirow{2}{*}{$\begin{array}{c}\text { Control } \\
\text { Device } \\
\text { Removal, } \\
\%\end{array}$} \\
\hline & & & & & Filter & Peroxide & Permanganate & Filter & Peroxide & Permanganate & & & & & \\
\hline $\begin{array}{l}\text { Bailly } \\
\text { Station, } \\
\text { M29 } \\
\end{array}$ & $\begin{array}{l}3.28 \\
2.54 \\
2.57 \\
\end{array}$ & 2.80 & 0.42 & $p$ & $\begin{array}{c}77-107 \\
P\end{array}$ & $\begin{array}{c}99 \\
106 \\
p\end{array}$ & $\begin{array}{c}87-106 \\
P\end{array}$ & NA & $\begin{array}{c}81 \\
142 \\
F\end{array}$ & NA & NA & NA & 2.1 & 0.78 & $\begin{array}{l}\text { ESP\#7, \#8 (-) } \\
\text { AFGD } 33.04\end{array}$ \\
\hline $\begin{array}{c}\text { Bailly } \\
\text { Station, } \\
\text { Brooks-Rand } \\
\text { Balds }\end{array}$ & $\begin{array}{l}3.48 \\
3.59 \\
3.50 \\
\end{array}$ & 3.52 & 0.06 & NA & NR & NR & $\mathrm{NR}$ & $\mathrm{NR}$ & NR & $\mathrm{NR}$ & NA & NA & 2.6 & 0.16 & $\begin{array}{c}\text { ESP } 1-1 \\
\text { AFGD. } 52.68 \\
\end{array}$ \\
\hline $\begin{array}{c}\text { Baldwin } \\
\text { Power Station, } \\
\text { M29 } \\
\text { Non-Sootblowing } \\
\end{array}$ & $\begin{array}{l}6.15 \\
5.27 \\
4.23 \\
\end{array}$ & 5.22 & 0.96 & $P$ & NA & NA & $\begin{array}{c}104 \\
P\end{array}$ & NA & $\begin{array}{l}84 \\
78 \\
\mathrm{P} \\
\end{array}$ & NA & $p$ & $P$ & 3.8 & 1.7 & ESP 26 \\
\hline $\begin{array}{c}\text { Baldwin } \\
\text { Power Station, } \\
\text { M29 } \\
\text { Sootblowing } \\
\end{array}$ & $\begin{array}{l}6 \\
7 \\
8 \\
\end{array}$ & 7 & 1 & $P$ & NA & NA & $\stackrel{104}{P}$ & NA & $\begin{array}{c}84, \\
78 \\
P\end{array}$ & NA & $p$ & $P$ & 5.4 & 2.4 & ESP 24.4 \\
\hline $\begin{array}{c}\text { Baldwin } \\
\text { Power Station, } \\
\text { MESA }\end{array}$ & $\begin{array}{l}6.95 \\
6.32 \\
8.30 \\
\end{array}$ & 7.19 & 1.01 & $P$ & NR & NR & NR & $\mathrm{NR}$ & NR & $\mathrm{NR}$ & NA & NA & 5.3 & 1.9 & ESP 15.7 \\
\hline $\begin{array}{c}\text { Boswell } \\
\text { Energy Center, } \\
\text { M29 }\end{array}$ & $\begin{array}{l}2.21 \\
1.82 \\
3.68\end{array}$ & 2.57 & 1.00 & $P$ & NA & $\stackrel{102}{P}$ & NA & NA & NA & $\underset{P}{98-102}$ & $\mathbf{P}$ & $p$ & 1.9 & 1.8 & FF 60 \\
\hline $\begin{array}{c}\text { Boswell } \\
\text { Energy Center, } \\
\text { MESA }\end{array}$ & $\begin{array}{l}2.9 \\
2.2 \\
4.3\end{array}$ & 3.1 & 1.1 & $p$ & NR & NR & NR & NR & $\mathrm{NR}$ & NR & NA & NA & 2,4 & 2.1 & 52 \\
\hline $\begin{array}{c}\text { Cardinal } \\
\text { Plant, } \\
\text { M29 } \\
\text { Non-Sootblowing }\end{array}$ & $\begin{array}{l}0.214 \\
0.927 \\
0.632 \\
0.481\end{array}$ & 0.563 & 0.298 & $P$ & NA & NA & NA & NA & NA & $\begin{array}{l}10 \\
F\end{array}$ & $P$ & $F$ & 0.44 & 0.40 & \\
\hline $\begin{array}{c}\text { Cardinal } \\
\text { Plant } \\
\text { M29 } \\
\text { Sootblowing } \\
\end{array}$ & $\begin{array}{l}7.02 \\
0.986 \\
0.991 \\
\end{array}$ & 1.0 & 0.02 & $p$ & NA & NA & $\mathrm{NA}$ & NA & NA & $\begin{array}{l}10 \\
F\end{array}$ & $P$ & $F$ & 0.78 & 0.37 & NA \\
\hline $\begin{array}{c}\text { Cardinal } \\
\text { Plant } \\
\text { Carbon Trap }\end{array}$ & $\begin{array}{c}6.17 \\
14.6 \\
12\end{array}$ & 10.9 & 4.3 & $P$ & NR & $\mathrm{NR}$ & NR & NR & NR & $\mathrm{NR}$ & $P$ & $P$ & 8.5 & 9.3 & ESP 18.6 \\
\hline $\begin{array}{c}\text { Coal Creek } \\
\text { Station, } \\
\text { M29 }\end{array}$ & $\begin{array}{l}11.2 \\
17.1 \\
9.74 \\
\end{array}$ & 12.7 & 3.9 & $\begin{array}{c}0.532 \\
15.5 \\
F\end{array}$ & & $\underset{p}{94-100}$ & & NA & NA & $\begin{aligned} 7,153 \\
F\end{aligned}$ & $p$ & $P$ & 9.5 & 7.5 & $\begin{array}{c}\text { ESPF1 } 14.4 \\
\text { Wet FGO }-8.9 \\
\text { Combined }-0.9\end{array}$ \\
\hline $\begin{array}{l}\text { Coal Creek } \\
\text { Station, } \\
\text { HEST } \\
\end{array}$ & $\begin{array}{l}8.64 \\
7.39 \\
8.08 \\
\end{array}$ & 8.04 & 0.63 & NA & $\mathrm{NR}$ & $\mathrm{NR}$ & $\mathrm{NR}$ & NR & NR & NR & NA & NA & NA & NA & NA \\
\hline $\begin{array}{c}\text { Niles } \\
\text { Station, } \\
\text { M29 } \\
\end{array}$ & $\begin{array}{l}27.4 \\
21.2 \\
23.2 \\
\end{array}$ & 23.9 & 3.2 & $\frac{0.028}{P}$ & & $\underset{p}{80-144}$ & & $\begin{array}{l}45 \\
47 \\
F\end{array}$ & $\begin{array}{l}84 \\
P\end{array}$ & $\begin{array}{c}124 \\
131 \\
F\end{array}$ & $P$ & $\mathrm{P}$ & 14 & 6.4 & ESP 29.92 \\
\hline $\begin{array}{c}\text { Niles } \\
\text { Station, } \\
\text { HEST }\end{array}$ & $\begin{array}{l}11.4 \\
36.8 \\
16.8 \\
\end{array}$ & 21.7 & 13.4 & NA & NR & $\mathrm{NR}$ & NR & NR & NR & NR & NA & NA & NA & NA & NA \\
\hline $\begin{array}{l}\text { Niles/SNOX } \\
\text { Station, } \\
\text { M29 }\end{array}$ & $\begin{array}{l}36.2 \\
21.9 \\
30.7 \\
\end{array}$ & 29.6 & 7.21 & $\underset{P}{0.251}$ & & $\underset{F}{80-144}$ & & $\begin{array}{l}45 \\
47 \\
F\end{array}$ & ${ }_{\mathrm{P}}^{84}$ & $\begin{array}{c}124 \\
131 \\
F\end{array}$ & $P$ & $p$ & 22 & 13 & $\begin{aligned} \text { FF }(-5) \\
\text { SNOX }(-13) \\
\end{aligned}$ \\
\hline $\begin{array}{l}\text { Niles/SNOX } \\
\text { Station, } \\
\text { HEST }\end{array}$ & $\begin{array}{l}30.2 \\
32.9\end{array}$ & 31.6 & 1.91 & NA & NR & NR & NR & NR & NR & NR & NA & NA & NA & NA & NA \\
\hline $\begin{array}{l}\text { Plant } \\
\text { Vates. } \\
\text { M29 }\end{array}$ & $\begin{array}{l}2.92 \\
3.14 \\
3.07\end{array}$ & 3.04 & 0.12 & $\mathrm{p}$ & $\stackrel{128}{F}$ & $\underset{p}{98}$ & $\begin{array}{l}33 \\
F\end{array}$ & $\begin{array}{l}73 \\
100 \\
P\end{array}$ & $\begin{array}{r}126.8, \\
113.4 \\
p\end{array}$ & $\begin{array}{c}20.9 \\
39.5 \\
F\end{array}$ & $P$ & $P$ & 3.0 & 0.3 & $\begin{array}{l}\text { ESP } 16.5 \\
\text { JBR } 45.9\end{array}$ \\
\hline $\begin{array}{c}\text { Plant } \\
\text { Yates, } \\
\text { MESA } \\
\end{array}$ & $\begin{array}{l}3.4 \\
3.6 \\
2.9 \\
\end{array}$ & 3.30 & 0.36 & $p$ & $\mathrm{NR}$ & $\mathrm{NR}$ & NR & NR & $\mathrm{NB}$ & $\mathrm{NR}$ & $p$ & $p$ & NA & $N A$ & $\begin{array}{l}E S P(-) \\
\operatorname{SBR} 54,6\end{array}$ \\
\hline $\begin{array}{c}\text { Springerville } \\
\text { Generating } \\
\text { Station, } \\
\text { M29, }\end{array}$ & $\begin{array}{l}11.4 \\
9.02 \\
8.51 \\
\end{array}$ & 9.64 & 7.54 & $\mathrm{NA}$ & $\begin{array}{c}110, \\
101 \\
P\end{array}$ & $\begin{array}{c}116, \\
121 \\
P\end{array}$ & $\begin{array}{c}138, \\
143 \\
F \\
\end{array}$ & NA & NA & $\begin{array}{c}95.8_{1} \\
111.3 \\
P .3\end{array}$ & $\mathrm{NA}$ & NA & NA & NA & $\begin{array}{l}\text { FF } 62 \pm 40 \\
\text { SDA-FF (-) } \\
\end{array}$ \\
\hline $\begin{array}{c}\text { Springerville } \\
\text { Generating } \\
\text { Station, } \\
\text { Brooks-Band }\end{array}$ & $\begin{array}{l}6.02 \\
6.88 \\
4.92\end{array}$ & 5.94 & 0.98 & $\mathrm{NA}$ & $\mathrm{NR}$ & $\mathrm{NR}$ & $\mathrm{NR}$ & $\mathrm{NR}$ & $N R$ & NR & NA & NA & 40 & 1.6 & $\begin{array}{l}\text { FF } 17.5 \\
\text { SOA-FF } 22.0\end{array}$ \\
\hline
\end{tabular}

NA - Not availab ND - Nondetect NR - Not required P - Pass F Fail $x$ - Value invalid because of high background level.

$y$-Value considered an outlier. Note: A detailed discussion of QA/OC criteria is presented in Section 4.2.1. 
Table A-15. Total Manganese

\begin{tabular}{|c|c|c|c|c|c|c|c|c|c|c|c|c|}
\hline \multirow{2}{*}{$\begin{array}{c}\text { Station/ } \\
\text { Plant } \\
\text { Name }\end{array}$} & \multirow{2}{*}{$\begin{array}{c}\text { Total } \\
\text { Stack } \\
\text { Conc., } \\
\mu \mathrm{g} / \mathrm{Nm}^{3}\end{array}$} & \multirow{2}{*}{$\begin{array}{l}\text { Total } \\
\text { Mean } \\
\text { Conc.., } \\
\mu \mathrm{g} / \mathrm{Nm}^{3}\end{array}$} & \multirow{2}{*}{$\begin{array}{l}\text { Standard } \\
\text { Deviation }\end{array}$} & \multirow{2}{*}{$\begin{array}{l}\text { Blank } \\
\text { Conc. } \\
\text { Range, } \\
\mu \mathrm{g} / \mathrm{Nm}^{3}\end{array}$} & \multicolumn{2}{|c|}{ M29 Internal Spike Recovery, \% } & \multirow{2}{*}{$\begin{array}{c}\text { M29 } \\
\text { Audit Spike } \\
\text { Recovery. } \\
\%\end{array}$} & \multirow[b]{2}{*}{$\begin{array}{c}\text { Above } \\
\text { DL }\end{array}$} & \multirow[b]{2}{*}{$\begin{array}{c}\text { Above } \\
\text { LLO }\end{array}$} & \multirow{2}{*}{$\begin{array}{l}\text { Emission } \\
\text { Factor, } \\
\mathrm{Ib} / 10^{12} \mathrm{Btu}\end{array}$} & \multirow{2}{*}{$\begin{array}{c}\text { Emission } \\
\text { Factor } \\
95 \% \mathrm{Cl}\end{array}$} & \multirow{2}{*}{$\begin{array}{c}\text { Control } \\
\text { Device } \\
\text { Removal, } \\
\%\end{array}$} \\
\hline & & & & & Filter & Peroxide & & & & & & \\
\hline $\begin{array}{c}\text { Bailly } \\
\text { Station, } \\
\text { M29 }\end{array}$ & $\begin{array}{l}3.17 \\
3.19 \\
2.88\end{array}$ & 3.08 & 0.17 & $\mathrm{~F}$ & $\begin{array}{c}97.5 \\
99.2 \\
P\end{array}$ & $\begin{array}{l}93.9, \\
95.1 \\
P\end{array}$ & NA & NA & NA & 3.1 & 0.42 & $\begin{array}{l}\text { ESP\#7 } 99.22 \\
\text { ESP\#8 } 99.85 \\
\text { AFGD } 62 \pm 17\end{array}$ \\
\hline $\begin{array}{c}\text { Baldwin } \\
\text { Power Station, } \\
\text { M29 } \\
\text { Non-Sootblowing }\end{array}$ & $\begin{array}{l}32 \\
30 \\
29\end{array}$ & 30 & 2 & $\mathbf{P}$ & NA & NA & NA & $\mathbf{P}$ & $\mathbf{P}$ & 22 & 2.7 & ESP 97 \\
\hline $\begin{array}{c}\text { Baldwin } \\
\text { Power Station, } \\
\text { M29 } \\
\text { Sootblowing }\end{array}$ & $\begin{array}{l}39 \\
53 \\
39 \\
\end{array}$ & 43 & 8 & $\mathbf{P}$ & NA & NA & NA & $p$ & $\mathbf{P}$ & 32 & 14 & ESP 97 \\
\hline $\begin{array}{c}\text { Boswell } \\
\text { Energy Center, } \\
\text { M29 }\end{array}$ & $\begin{array}{l}15.06 \\
17.72 \\
40.88 \\
\end{array}$ & 24.55 & 14.2 & $\mathrm{P}$ & NA & NA & NA & $\mathrm{P}$ & $\mathrm{F}$ & 18 & 26 & FF $>99$ \\
\hline $\begin{array}{c}\text { Cardinal } \\
\text { Plant, } \\
\text { M29 } \\
\text { Non-Sootblowing }\end{array}$ & $\begin{array}{l}1.46 \\
62.1 \\
2.81 \\
10.9\end{array}$ & 19.3 & 28.82 & $\mathrm{~F}$ & $\begin{array}{c}76 \\
P\end{array}$ & $\begin{array}{c}77 \\
P\end{array}$ & NA & $P$ & $\mathbf{P}$ & 15 & 36 & \\
\hline $\begin{array}{l}\text { Cardinal } \\
\text { Plant, } \\
\text { M29 } \\
\text { Sootblowing }\end{array}$ & $\begin{array}{l}7.31 \\
2.62 \\
69.4\end{array}$ & 26.4 & 37.27 & $\mathrm{~F}$ & $\begin{array}{c}76 \\
P\end{array}$ & $\begin{array}{c}77 \\
p\end{array}$ & NA & $\mathrm{P}$ & $\mathbf{P}$ & 20 & 72 & 201 00.00 \\
\hline $\begin{array}{c}\text { Coal Creek } \\
\text { Station, } \\
\text { M29 }\end{array}$ & $\begin{array}{l}55.8 \\
26.8 \\
37.7\end{array}$ & 40.1 & 14.6 & $\begin{array}{c}N D<2.23 \\
N D<6.09 \\
P\end{array}$ & $\frac{83-102}{P}$ & $\begin{array}{c}94.7 \\
P\end{array}$ & NA & $p$ & $\mathbf{P}$ & 30 & 26 & $\begin{array}{c}\text { ESP\#1 } 99.7 \\
\text { Wet FGD } 5.1 \\
\text { Combined } 99.6\end{array}$ \\
\hline $\begin{array}{l}\text { Niles } \\
\text { Station, } \\
\text { M29 }\end{array}$ & $\begin{array}{l}7.66 \\
4.09 \\
5.07\end{array}$ & 5.61 & 1.84 & $\stackrel{2.55}{F}$ & \multicolumn{2}{|c|}{$\begin{array}{c}89-136 \\
P\end{array}$} & NA & $p$ & $\mathrm{P}$ & 3.4 & 3.1 & ESP 98.98 \\
\hline $\begin{array}{l}\text { Niles/SNOX } \\
\text { Station, } \\
\text { M29 }\end{array}$ & $\begin{array}{l}2.45 \\
5.60 \\
2.85\end{array}$ & 3.63 & 1.71 & $\begin{array}{l}1.7 \\
\mathrm{~F}\end{array}$ & \multicolumn{2}{|c|}{$\begin{array}{c}89-136 \\
P\end{array}$} & NA & $p$ & $\mathrm{~F}$ & 2.6 & 3.1 & $\begin{array}{c}\text { FF } 99.45 \\
\text { SNOX } 99.34\end{array}$ \\
\hline $\begin{array}{l}\text { Plant } \\
\text { Yates, } \\
\text { M29 }\end{array}$ & $\begin{array}{c}2.94^{x} \\
3.46 \\
11.11\end{array}$ & 7.28 & 5.41 & $\stackrel{10.6}{F}$ & $\begin{array}{c}94 \\
P\end{array}$ & $\begin{array}{c}104 \\
P\end{array}$ & NA & $P$ & $\mathrm{~F}$ & 7.2 & 48 & $\begin{array}{l}\text { ESP } 98.4 \\
\text { JBR } 78.4\end{array}$ \\
\hline $\begin{array}{l}\text { Springerville } \\
\text { Generating } \\
\text { Station, } \\
\text { M29 }\end{array}$ & $\begin{array}{l}45.9^{y} \\
14.9 \\
17.4\end{array}$ & 16.0 & 1.9 & NA & $\begin{array}{c}84 \\
100 \\
P\end{array}$ & $\begin{array}{c}104 \\
99 \\
\mathrm{P}\end{array}$ & NA & NA & NA & 11 & 12 & $\begin{array}{c}\text { FF } 99.93 \\
\text { SDA.FF } 99.80\end{array}$ \\
\hline
\end{tabular}

NA - Not available. ND - Nondetect. NR - Not required. P - Pass. F - Fail. $x$ - Value invalid because of high background level.

$y$-Value considered an outlier. Note: A detailed discussion of QA/QC criteria is presented in Section 4.2.1. 
$\rightarrow$ Table A-16. Total Molybdenum

\begin{tabular}{|c|c|c|c|c|c|c|c|c|c|c|c|c|}
\hline \multirow{2}{*}{$\begin{array}{c}\text { Station/ } \\
\text { Plant } \\
\text { Name }\end{array}$} & \multirow{2}{*}{$\begin{array}{c}\text { Total } \\
\text { Stack } \\
\text { Conc., } \\
\mu \mathrm{g} / \mathrm{Nm}^{3}\end{array}$} & \multirow{2}{*}{$\begin{array}{c}\text { Total } \\
\text { Mean } \\
\text { Conc., } \\
\mu g / \mathrm{Nm}^{3}\end{array}$} & \multirow[b]{2}{*}{$\begin{array}{l}\text { Standard } \\
\text { Deviation }\end{array}$} & \multirow{2}{*}{$\begin{array}{l}\text { Blank } \\
\text { Conc. } \\
\text { Range, } \\
\mu \mathrm{g} / \mathrm{Nm}^{3}\end{array}$} & \multicolumn{2}{|c|}{ M29 Internal Spike Recovery, \% } & \multirow{2}{*}{$\begin{array}{c}\text { M29 } \\
\text { Audit Spike } \\
\text { Recovery, } \\
\%\end{array}$} & \multirow[b]{2}{*}{$\begin{array}{c}\text { Above } \\
\text { DL }\end{array}$} & \multirow[b]{2}{*}{$\begin{array}{l}\text { Above } \\
\text { LLO }\end{array}$} & \multirow{2}{*}{$\begin{array}{l}\text { Emission } \\
\text { Factor, } \\
\text { lb/10 }\end{array}$} & \multirow{2}{*}{$\begin{array}{c}\text { Emission } \\
\text { Factor } \\
\mathbf{9 5 \%} \mathrm{Cl}\end{array}$} & \multirow{2}{*}{$\begin{array}{c}\text { Control } \\
\text { Device } \\
\text { Removal, } \\
\%\end{array}$} \\
\hline & & & & & Filter & Peroxide & & & & & & \\
\hline $\begin{array}{l}\text { Bailly } \\
\text { Station, } \\
\text { M29 }\end{array}$ & $\begin{array}{l}4.90 \\
4.22 \\
4.73\end{array}$ & 4.61 & 0.35 & $\mathrm{~F}$ & $\begin{array}{l}90.8 \\
97.5 \\
P\end{array}$ & $\begin{array}{l}95.6 \\
95.8 \\
P\end{array}$ & NA & NA & NA & 3.4 & 0.65 & $\begin{array}{l}\text { ESP\#7 } 97.72 \\
\text { ESPH8 } 99.38 \\
\text { AFGD } 49.92\end{array}$ \\
\hline $\begin{array}{c}\text { Baldwin } \\
\text { Power Station, } \\
\text { M29 } \\
\text { Non-Sootblowing }\end{array}$ & $\begin{array}{l}50 \\
50 \\
38 \\
\end{array}$ & 46 & 7 & $\mathrm{P}$ & NA & NA & NA & NA & NA & 34 & 13 & ESP 91 \\
\hline $\begin{array}{c}\text { Baldwin } \\
\text { Power Station, } \\
\text { M29 } \\
\text { Sootblowing }\end{array}$ & $\begin{array}{l}71 \\
54 \\
43\end{array}$ & 56 & 14 & $\mathbf{P}$ & NA & NA & NA & NA & NA & 41 & 23 & ESP 93 \\
\hline $\begin{array}{c}\text { Boswell } \\
\text { Energy Center, } \\
\text { M29 }\end{array}$ & $\begin{array}{l}0.78 \\
0.30 \\
4.09\end{array}$ & 1.72 & 2.06 & $\mathbf{P}$ & NA & NA & NA & NA & NA & 1.3 & 3.8 & $\mathrm{FF}>99$ \\
\hline $\begin{array}{c}\text { Cardinal } \\
\text { Plant. } \\
\text { M29 } \\
\text { Non-Sootblowing }\end{array}$ & $\begin{array}{l}0.469 \\
1.13 \\
1.48 \\
0.375\end{array}$ & 0.863 & 0.531 & $\mathrm{~F}$ & $\begin{array}{c}61 \\
F\end{array}$ & $\begin{array}{c}61 \\
F\end{array}$ & NA & NA & NA & 0.59 & 0.84 & \\
\hline $\begin{array}{l}\text { Cardinal } \\
\text { Plant, } \\
\text { M29 } \\
\text { Sootblowing }\end{array}$ & $\begin{array}{l}0.555 \\
0.416 \\
0.563\end{array}$ & 0.511 & 0.0827 & $\mathrm{~F}$ & $\begin{array}{l}61 \\
F\end{array}$ & $\begin{array}{c}61 \\
F\end{array}$ & NA & NA & NA & 0.27 & 0.38 & \\
\hline $\begin{array}{l}\text { Coal Creek } \\
\text { Station, } \\
\text { M29 }\end{array}$ & $\begin{array}{l}9.68^{v} \\
0.67 \\
0.70\end{array}$ & 0.68 & 0.02 & $\begin{array}{c}\text { ND }<0.154 \\
\text { ND }<0.421 \\
P\end{array}$ & $\begin{array}{c}76-102 \\
P\end{array}$ & $\underset{P}{101.6}$ & NA & $\mathrm{F}$ & $\mathrm{F}$ & 0.51 & 0.14 & $\begin{array}{c}\text { ESP\#1 } 199.3 \\
\text { Wet FGD 54.1 } \\
\text { Combined } 99.7\end{array}$ \\
\hline $\begin{array}{l}\text { Niles } \\
\text { Station, } \\
\text { M29 }\end{array}$ & $\begin{array}{l}4.09 \\
4.27 \\
2.87\end{array}$ & 3.74 & 0.76 & $\stackrel{3.01}{F}$ & \multicolumn{2}{|c|}{$\begin{array}{c}97-144 \\
F\end{array}$} & NA & $\mathrm{P}$ & $\mathrm{F}$ & 2.3 & 1.3 & ESP 98.09 \\
\hline $\begin{array}{l}\text { Niles/SNOX } \\
\text { Station, } \\
\text { M29 }\end{array}$ & $\begin{array}{l}6.90 \\
9.64 \\
5.88\end{array}$ & 7.47 & 1.94 & ${ }_{F}^{3.71}$ & \multicolumn{2}{|c|}{$\begin{array}{c}97-144 \\
F\end{array}$} & NA & $P$ & $\mathbf{F}$ & 5.4 & 3.6 & $\begin{array}{c}\text { FF } 99.04 \\
\text { SNOX } 96.92\end{array}$ \\
\hline $\begin{array}{l}\text { Plant } \\
\text { Yates, } \\
\text { M29 }\end{array}$ & $\begin{array}{l}0.40^{*} \\
1.32 \\
1.70\end{array}$ & 1.51 & 0.27 & $\stackrel{0.073}{P}$ & $\begin{array}{c}94 \\
P\end{array}$ & $\begin{array}{c}100 \\
P\end{array}$ & NA & $\mathbf{P}$ & $\mathbf{F}$ & 1.5 & 2.6 & $\begin{array}{c}\text { ESP } 97.2 \\
\text { JBR } \\
82.5 \pm 27.2\end{array}$ \\
\hline $\begin{array}{l}\text { Springerville } \\
\text { Generating } \\
\text { Station, } \\
\text { M29 }\end{array}$ & $\begin{array}{l}1.93 \\
2.18 \\
2.0\end{array}$ & 2.04 & 0.13 & NA & $\begin{array}{c}101, \\
104 \\
P\end{array}$ & $\begin{array}{l}95, \\
96 \\
P\end{array}$ & NA & NA & NA & 1.4 & 0.2 & $\begin{array}{c}\text { FF } 99.54 \\
\text { SDA.FF } 98.1\end{array}$ \\
\hline
\end{tabular}

NA - Not available. ND - Nondetect. NR - Not required. P-Pass. F - Fail. $x$ - Value invalid because of high background level.

$y$-Value considered an outlier. Note: A detailed discussion of QA/QC criteria is presented in Section 4.2.1. 
Table A-17. Total Ammonia

\begin{tabular}{|c|c|c|c|c|c|c|c|c|c|c|c|}
\hline $\begin{array}{c}\text { Station/ } \\
\text { Plant } \\
\text { Name }\end{array}$ & $\begin{array}{c}\text { Total } \\
\text { Stack } \\
\text { Conc.. } \\
\mu \mathrm{g} / \mathrm{Nm}^{3}\end{array}$ & $\begin{array}{c}\text { Total } \\
\text { Mean } \\
\text { Conc.., } \\
\mu \mathrm{g} / \mathrm{Nm}^{3}\end{array}$ & $\begin{array}{l}\text { Standard } \\
\text { Deviation }\end{array}$ & $\begin{array}{l}\text { Blank } \\
\text { Conc. } \\
\text { Range, } \\
\mu g / \mathrm{Nm}^{3}\end{array}$ & $\begin{array}{c}\text { Internal } \\
\text { Spike } \\
\text { Recovery. } \\
\%\end{array}$ & $\begin{array}{c}\text { Audit } \\
\text { Spike } \\
\text { Recovery, } \\
\%\end{array}$ & $\begin{array}{c}\text { Above } \\
\text { DL }\end{array}$ & $\begin{array}{l}\text { Above } \\
\text { LLO }\end{array}$ & $\begin{array}{l}\text { Emission } \\
\text { Factor, } \\
\mid \mathrm{b} / 10^{12} \text { Btu }\end{array}$ & $\begin{array}{l}\text { Emission } \\
\text { Factor } \\
95 \% \mathrm{Cl}\end{array}$ & $\begin{array}{c}\text { Control } \\
\text { Device } \\
\text { Removal, } \\
\%\end{array}$ \\
\hline $\begin{array}{c}\text { Bailly } \\
\text { Station }\end{array}$ & 20.2 & NA & NA & NA & $\begin{array}{l}97 \\
P\end{array}$ & NA & NA & NA & NA & NA & NA \\
\hline $\begin{array}{l}\text { Baldwin } \\
\text { Power } \\
\text { Station }\end{array}$ & $\begin{array}{c}25.9 \\
\text { ND }<22.6 \\
\text { ND }<20.5\end{array}$ & ND $<22.6$ & 8.8 & NA & $P$ & NA & $\mathbf{F}$ & $F$ & ND $<17$ & 17 & $E S P>63$ \\
\hline $\begin{array}{l}\text { Boswell } \\
\text { Energy } \\
\text { Center }\end{array}$ & $\begin{array}{l}N D<20.7 \\
N D<24.8 \\
N D<21.0\end{array}$ & $N D<22.1$ & 2.28 & NA & $P$ & NA & $\mathbf{F}$ & $\mathbf{F}$ & $N D<17$ & 17 & $E S P>56$ \\
\hline $\begin{array}{l}\text { Cardinal } \\
\text { Plant }\end{array}$ & $\begin{array}{l}64.3 \\
28.6 \\
64.9\end{array}$ & 52.6 & 3.96 & NA & $P$ & NA & NA & NA & 40.7 & 7.6 & ESP NA \\
\hline $\begin{array}{l}\text { Coal Creek } \\
\text { Station }\end{array}$ & $\begin{array}{l}N D<5.06 \\
N D<4.85 \\
\text { ND }<5.13\end{array}$ & ND $<5.0$ & 0.15 & $\begin{array}{c}114 \\
1340 \\
F\end{array}$ & $\begin{array}{c}99 \\
125 \\
P\end{array}$ & NA & $\mathrm{F}$ & $\mathrm{F}$ & ND $<3.7$ & 3.7 & NA \\
\hline $\begin{array}{c}\text { Niles } \\
\text { Station }\end{array}$ & $\begin{array}{c}N D<1.15 \\
352 \\
N D<1.21\end{array}$ & 118 & 203 & $\underset{\mathrm{ND}}{\mathrm{ND}<1.30}$ & $\underset{P}{100-104}$ & NA & $\mathrm{F}$ & $\mathrm{F}$ & 70 & 298 & NA \\
\hline $\begin{array}{l}\text { Niles/SNOX } \\
\text { Station }\end{array}$ & $\begin{array}{l}590^{\prime} \\
67.1 \\
86.4\end{array}$ & 76.8 & 13.6 & $\stackrel{20.5}{F}$ & $\underset{P}{100-104}$ & NA & $\mathbf{F}$ & $\mathrm{F}$ & 56 & 89 & NA \\
\hline $\begin{array}{l}\text { Plant } \\
\text { Yates }\end{array}$ & $\begin{array}{l}18.72 \\
5.91 \\
8.98\end{array}$ & 11.20 & 6.69 & NA & $\begin{array}{c}63 \\
F\end{array}$ & NA & NA & NA & NA & NA & NA \\
\hline $\begin{array}{l}\text { Springerville } \\
\text { Generating } \\
\text { Station }\end{array}$ & $\begin{array}{l}680 \\
52 \\
52\end{array}$ & 261 & 363 & NA & NA & NA & NA & NA & 174 & 601 & NA \\
\hline
\end{tabular}

NA - Not available, ND - Nondetect. NR - Not required, P - Pass. F - Fail. $x$ - Value invalid because of high background level.

$y$-Value considered an outlier. Note: A detailed discussion of $\mathrm{QA} / \mathrm{OC}$ criteria is presented in Section 4.2.1. 
Table A-18. Total Nickel

\begin{tabular}{|c|c|c|c|c|c|c|c|c|c|c|c|c|}
\hline \multirow{2}{*}{$\begin{array}{l}\text { Station/ } \\
\text { Plant, } \\
\text { Name }\end{array}$} & \multirow{2}{*}{$\begin{array}{c}\text { Total } \\
\text { Stack } \\
\text { Concs., } \\
\mu \mathrm{g} / \mathrm{Nm}^{3}\end{array}$} & \multirow{2}{*}{$\begin{array}{c}\text { Total } \\
\text { Mean } \\
\text { Conc.. } \\
\mu \mathrm{g} / \mathrm{Nm}^{3}\end{array}$} & \multirow[b]{2}{*}{$\begin{array}{l}\text { Standard } \\
\text { Deviation }\end{array}$} & \multirow{2}{*}{$\begin{array}{l}\text { Blank } \\
\text { Conc. } \\
\text { Range, } \\
\mu g / \mathrm{Nm}^{3}\end{array}$} & \multicolumn{2}{|c|}{$\begin{array}{c}\text { M29 Internal Spike Recovery, } \\
\%\end{array}$} & \multirow{2}{*}{$\begin{array}{l}\text { No M29 } \\
\text { Audit Spike } \\
\text { Performed }\end{array}$} & \multirow[b]{2}{*}{$\begin{array}{c}\text { Above } \\
\text { OL }\end{array}$} & \multirow[b]{2}{*}{$\begin{array}{l}\text { Above } \\
\text { LLQ }\end{array}$} & \multirow{2}{*}{$\begin{array}{l}\text { Emission } \\
\text { Factor, } \\
\text { Ib/110' Btu }\end{array}$} & \multirow{2}{*}{$\begin{array}{l}\text { Emission } \\
\text { Factor } \\
95 \% \mathrm{Cl}\end{array}$} & \multirow{2}{*}{$\begin{array}{c}\text { Control } \\
\text { Device } \\
\text { Removal, } \\
\%\end{array}$} \\
\hline & & & & & Filter & Peroxide & & & & & & \\
\hline $\begin{array}{l}\text { Bailly } \\
\text { Station, } \\
\text { M29 }\end{array}$ & $\begin{array}{l}2.07 \\
2.88 \\
3.24\end{array}$ & 2.92 & 0.61 & $F$ & $\begin{array}{c}103.6 \\
102.1 \\
P\end{array}$ & $\begin{array}{c}92.5 \\
90.4 \\
P\end{array}$ & NA & NA & NA & 2.2 & 1.1 & $\begin{array}{r}\text { ESP\#7 } 98.63 \\
\text { ESP \#8 99.45 } \\
\text { AFGD } 70 \pm 12\end{array}$ \\
\hline $\begin{array}{c}\text { Baldwin } \\
\text { Power Station, } \\
\text { M29 } \\
\text { Non-Sootblowing }\end{array}$ & $\begin{array}{l}31 \\
32 \\
28\end{array}$ & 30 & 2 & $\mathbf{P}$ & NA & NA & NA & P & $P$ & 22 & 3.2 & ESP 97 \\
\hline $\begin{array}{c}\text { Baldwin } \\
\text { Power Station. } \\
\text { M29 } \\
\text { Sootblowing }\end{array}$ & $\begin{array}{l}35 \\
51 \\
40\end{array}$ & 42 & 8 & $\mathrm{P}$ & NA & NA & NA & $\mathrm{P}$ & $P$ & 31 & 15 & ESP 97 \\
\hline $\begin{array}{c}\text { Boswell } \\
\text { Energy Center. } \\
\text { M29 }\end{array}$ & $\begin{array}{l}4.15 \\
1.43 \\
2.20\end{array}$ & 2.59 & 1.40 & $\mathrm{P}$ & NA & NA & NA & $\mathrm{P}$ & $\mathrm{F}$ & 2.0 & 2.8 & $F F>99$ \\
\hline $\begin{array}{c}\text { Cardinal } \\
\text { Plant, } \\
\text { M29 } \\
\text { Non-Sootblowing }\end{array}$ & $\begin{array}{l}1.71 \\
13.4 \\
6.69 \\
2.78\end{array}$ & 6.14 & 5.28 & $\mathrm{~F}$ & $\begin{array}{c}76 \\
P\end{array}$ & $\begin{array}{c}70 \\
P\end{array}$ & NA & $P$ & $P$ & 4.8 & 6.8 & \\
\hline $\begin{array}{c}\text { Cardinal } \\
\text { Plant. } \\
\text { M29 } \\
\text { Sootblowing }\end{array}$ & $\begin{array}{l}2.07 \\
2.52 \\
2.16\end{array}$ & 2.25 & 0.238 & $\mathrm{~F}$ & $\begin{array}{c}76 \\
P\end{array}$ & $\begin{array}{c}70 \\
p\end{array}$ & NA & $\mathrm{P}$ & $\mathrm{P}$ & 1.8 & 1.0 & - \\
\hline $\begin{array}{c}\text { Coal Creek } \\
\text { Station. } \\
\text { M29 }\end{array}$ & $\begin{array}{l}205^{\prime} \\
3.16 \\
10.6\end{array}$ & 6.88 & 5.26 & $\begin{array}{c}N D<1.93 \\
N D<5.26 \\
F\end{array}$ & $\begin{array}{c}87-117 \\
P\end{array}$ & $\begin{array}{c}106.4 \\
P\end{array}$ & NA & $P$ & $\mathrm{~F}$ & 5.1 & 35 & $\begin{array}{c}\text { ESP\#1 } 99.1 \\
\text { Wet FGD } 2.7 \\
\text { Combined } 99.6\end{array}$ \\
\hline $\begin{array}{l}\text { Niles } \\
\text { Station, } \\
\text { M29 }\end{array}$ & $\begin{array}{l}1.32 \\
0.93 \\
0.47\end{array}$ & 0.91 & 0.43 & $\begin{array}{l}3.07 \\
F\end{array}$ & \multicolumn{2}{|c|}{$\begin{array}{c}61-122 \\
F\end{array}$} & NA & $F$ & $\mathbf{F}$ & 0.55 & 0.69 & ESP 99.88 \\
\hline $\begin{array}{l}\text { Niles/SNOX } \\
\text { Station, } \\
\text { M29 }\end{array}$ & $\begin{array}{l}1.04 \\
33.1^{4} \\
5.01\end{array}$ & 3.02 & 2.81 & $\begin{array}{c}N D<0.219 \\
P\end{array}$ & \multicolumn{2}{|c|}{$\begin{array}{c}61-122 \\
F\end{array}$} & NA & $\mathrm{P}$ & $\mathbf{F}$ & 2.2 & 18 & $\begin{array}{c}\text { FF } 99.95 \\
\text { SNOX } 99.56\end{array}$ \\
\hline $\begin{array}{c}\text { Plant } \\
\text { Yates, } \\
\text { M29 }\end{array}$ & $\begin{array}{c}9.53^{x} \\
7.44 \\
75.52\end{array}$ & 41.48 & 48.14 & $\begin{array}{c}3.59 \\
F\end{array}$ & $\begin{array}{l}89 \\
P\end{array}$ & $\stackrel{102}{P}$ & NA & $\mathrm{P}$ & $\mathrm{F}$ & 40.1 & 435 & $\begin{array}{l}\text { ESP } 98.8 \\
\text { JBR }-75.7\end{array}$ \\
\hline $\begin{array}{c}\text { Springerville } \\
\text { Generating } \\
\text { Station, } \\
\text { M29 }\end{array}$ & $\begin{array}{l}N D<0.4 \\
N D<0.4 \\
N D<0.4\end{array}$ & $N D<0.4$ & NA & NA & $\begin{array}{c}88, \\
92 \\
\mathrm{P}\end{array}$ & $\begin{array}{l}96 . \\
96 \\
P\end{array}$ & NA & NA & NA & $\mathrm{ND}<0.3$ & 0.3 & $\begin{array}{c}\mathrm{FF}>99.97 \\
\text { SDA- } \\
\mathrm{FF}>99.94\end{array}$ \\
\hline
\end{tabular}

NA - Not available. ND - Nondetect. NR - Not required. $P$ - Pass. F - Fail. $x$ - Value invalid because of high background level.

$y$ - Value considered an outlier. Note: A detailed discussion of QA/QC criteria is presented in Section 4.2.1. 
Table A-19. Total Selenium

\begin{tabular}{|c|c|c|c|c|c|c|c|c|c|c|c|c|c|}
\hline \multirow{2}{*}{$\begin{array}{c}\text { Station/ } \\
\text { Ptant } \\
\text { Name }\end{array}$} & \multirow{2}{*}{$\begin{array}{l}\text { Total } \\
\text { Stack } \\
\text { Conc.. } \\
\mu \mathrm{g} / \mathrm{Nm}^{3}\end{array}$} & \multirow{2}{*}{$\begin{array}{l}\text { Total } \\
\text { Mean } \\
\text { Conc., } \\
\mu g / \mathrm{Nm}^{3}\end{array}$} & \multirow[b]{2}{*}{$\begin{array}{l}\text { Standard } \\
\text { Deviation }\end{array}$} & \multirow{2}{*}{$\begin{array}{l}\text { Blank } \\
\text { Conc. } \\
\text { Range, } \\
\mu g / \mathrm{Nm}^{3}\end{array}$} & \multicolumn{2}{|c|}{$\begin{array}{c}29 \text { Internal Spike } \\
\text { Recovery, \% }\end{array}$} & \multicolumn{2}{|c|}{$\begin{array}{l}\text { M29 Audit Spike } \\
\text { Recovery, \% }\end{array}$} & \multirow[b]{2}{*}{$\begin{array}{c}\text { Above } \\
\text { DL }\end{array}$} & \multirow[b]{2}{*}{$\begin{array}{l}\text { Above } \\
\text { LLa }\end{array}$} & \multirow{2}{*}{$\begin{array}{l}\text { Emission } \\
\text { Factor, } \\
\mathrm{lb} / 10^{12} \mathrm{Btu}\end{array}$} & \multirow{2}{*}{$\begin{array}{l}\text { Emission } \\
\text { Factor } \\
95 \% \mathrm{Cl}\end{array}$} & \multirow{2}{*}{$\begin{array}{c}\text { Control } \\
\text { Device } \\
\text { Removal, } \\
\%\end{array}$} \\
\hline & & & & & Filter & Peroxide & Filter & Pẹroxide & & & & & \\
\hline $\begin{array}{c}\text { Bailly } \\
\text { Station, } \\
\text { M29 }\end{array}$ & $\begin{array}{l}174 \\
193 \\
415 \\
\end{array}$ & 261 & 134 & $\mathbf{P}$ & $\begin{array}{c}162 . \\
138 \\
F\end{array}$ & $\begin{array}{c}59.1 \\
61.9 \\
F\end{array}$ & $\begin{array}{l}76 \\
78 \\
P \\
\end{array}$ & $\begin{array}{c}69 \\
85 \\
F \\
\end{array}$ & NA & NA & 193 & 246 & $\begin{array}{c}\text { ESP\#7 } 11.7 \\
\text { ESP \#8 } 57.7 \\
\text { AFGD }-16 \pm 45 \\
\end{array}$ \\
\hline $\begin{array}{c}\text { Baldwin } \\
\text { Power Station, } \\
\text { M29 } \\
\text { Non-Sootblowing }\end{array}$ & $\begin{array}{l}251 \\
114 \\
166\end{array}$ & 177 & 69 & $\mathrm{p}$ & NA & $\begin{array}{c}0 \\
40 \\
F\end{array}$ & $\begin{array}{c}101, \\
98 \\
p\end{array}$ & $\begin{array}{l}13, \\
23 \\
F\end{array}$ & $P$ & $P$ & 130 & 120 & NA \\
\hline $\begin{array}{c}\text { Baldwin } \\
\text { Power Station, } \\
\text { M29 } \\
\text { Sootblowing }\end{array}$ & $\begin{array}{c}296 \\
169 \\
95\end{array}$ & 187 & 102 & $\mathbf{P}$ & NA & $\begin{array}{c}0 \\
40 \\
F\end{array}$ & $\begin{array}{c}101 . \\
98 \\
p\end{array}$ & $\begin{array}{l}13, \\
23 \\
F\end{array}$ & $\mathbf{P}$ & $\mathbf{P}$ & 140 & 180 & NA \\
\hline $\begin{array}{c}\text { Boswell } \\
\text { Energy Center, } \\
\text { M29 }\end{array}$ & $\begin{array}{l}4.33 \\
4.11 \\
4.44\end{array}$ & .4 .29 & 0.17 & $P$ & $\begin{array}{c}77.3 \\
P\end{array}$ & $\begin{array}{c}90.2 \\
P\end{array}$ & $\begin{array}{l}37 \\
67 \\
F\end{array}$ & $\begin{array}{c}48 \\
129 \\
\mathrm{P}\end{array}$ & $P$ & $p$ & 3.3 & 0.38 & FF 66 \\
\hline $\begin{array}{c}\text { Cardinal } \\
\text { Plant, } \\
\text { M29 } \\
\text { Non-Sootblowing }\end{array}$ & $\begin{array}{l}75.4 \\
148 \\
127 \\
130 \\
\end{array}$ & 120 & 31 & $P$ & NA & $\begin{array}{c}133 \\
F\end{array}$ & $\begin{array}{c}80 \\
120 \\
P\end{array}$ & $\begin{array}{c}49 \\
F\end{array}$ & $P$ & $P$ & 93 & 50 & \multirow{2}{*}{ ESP 19.03} \\
\hline $\begin{array}{c}\text { Cardinal } \\
\text { Plant, } \\
\text { M29 } \\
\text { Sootblowing } \\
\end{array}$ & $\begin{array}{l}49.4 \\
9.52 \\
106\end{array}$ & 54.97 & 48.48 & $P$ & $\mathrm{NA}$ & $\stackrel{133}{F}$ & $\begin{array}{c}80 \\
120 \\
P\end{array}$ & $\begin{array}{l}49 \\
F\end{array}$ & $P$ & $P$ & 65 & 66 & \\
\hline $\begin{array}{l}\text { Coal Creek } \\
\text { Station, } \\
\text { M29 }\end{array}$ & $\begin{array}{l}15.3 \\
11.0 \\
7.14\end{array}$ & 11.1 & 4.08 & $\begin{array}{c}0.440, \\
13.1 \\
F\end{array}$ & $\begin{array}{c}100 \\
P\end{array}$ & $\underset{P}{100-115}$ & $\begin{array}{l}56, \\
57 \\
F \\
\end{array}$ & NA & $\mathbf{P}$ & $P$ & 8.3 & 7.4 & $\begin{array}{c}E S P \# 187.2 \\
\text { Wet FGD } 39.7 \\
\text { Combined } 88.7\end{array}$ \\
\hline $\begin{array}{c}\text { Coal Creek } \\
\text { Station, } \\
\text { HEST }\end{array}$ & $\begin{array}{l}20.2 \\
14.1 \\
8.26\end{array}$ & 14.2 & 6.0 & NA & $\mathrm{NR}$ & NR & NR & NR & NA & NA & NA & $\mathrm{NA}$ & $\mathrm{NA}$ \\
\hline $\begin{array}{c}\text { Niles } \\
\text { Station, } \\
\text { M29 }\end{array}$ & $\begin{array}{l}136 \\
56.1 \\
113 \\
\end{array}$ & 102 & 41 & $\begin{array}{c}N D<0.689 \\
P\end{array}$ & \multicolumn{2}{|c|}{$\underset{p}{75-117}$} & $\begin{array}{l}78, \\
47 \\
F \\
\end{array}$ & $\begin{array}{l}44, \\
71 \\
F\end{array}$ & $P$ & $\mathbf{P}$ & 62 & 67 & ESP 7.6 \\
\hline $\begin{array}{c}\text { Niles } \\
\text { Station, } \\
\text { HEST }\end{array}$ & $\begin{array}{l}273.4 \\
500.3 \\
177.4\end{array}$ & 317.0 & 165.8 & NA & NR & NR & NR & NR & NA & NA & NA & NA & NA \\
\hline $\begin{array}{l}\text { Niles/SNOX } \\
\text { Station, } \\
\text { M29 }\end{array}$ & $\begin{array}{l}1.42 \\
0.610 \\
0.721\end{array}$ & 0.917 & 0.439 & $\begin{array}{c}1.43 \\
F\end{array}$ & \multicolumn{2}{|c|}{$\begin{array}{c}75-117 \\
P\end{array}$} & $\begin{array}{l}78, \\
47 \\
F \\
\end{array}$ & $\begin{array}{l}44, \\
71 \\
F\end{array}$ & $p$ & $\mathbf{F}$ & 0.67 & 0.80 & $\begin{array}{c}\text { FF } 17 \\
\text { SNOX } 99.12 \\
\end{array}$ \\
\hline $\begin{array}{l}\text { Niles/SNOX } \\
\text { Station, } \\
\text { HEST }\end{array}$ & $\begin{array}{l}N D<0.128 \\
N D<0.152\end{array}$ & $\mathrm{ND}<0.140$ & 0.017 & NA & $\mathrm{NR}$ & NR & NR & NR & NA & NA & NA & NA & NA \\
\hline $\begin{array}{c}\text { Plant } \\
\text { Yates, } \\
\text { M29 } \\
\end{array}$ & $\begin{array}{l}52.99 \\
10.60 \\
17.08 \\
\end{array}$ & 26.89 & 22.83 & $\begin{array}{c}N D<0.228 \\
P\end{array}$ & NA & $\begin{array}{l}0 \\
\mathrm{~F}\end{array}$ & $\begin{array}{c}89.2, \\
66.9 \\
F\end{array}$ & $\begin{array}{c}104 . \\
113.4 \\
P\end{array}$ & $P$ & $P$ & 26 & 58 & $\begin{array}{l}\text { ESP } 38.1 \\
\text { JBR } 66.9 \\
\end{array}$ \\
\hline $\begin{array}{l}\text { Springerville } \\
\text { Generating } \\
\text { Station, } \\
\text { M29 }\end{array}$ & $\begin{array}{l}\text { ND }<0.01 \\
\text { ND }<0.054 \\
\text { ND }<0.28^{*}\end{array}$ & $N D<0.032$ & 0.031 & NA & $\begin{array}{c}91 . \\
97 \\
P\end{array}$ & NA & $\begin{array}{c}108 \\
105 \\
p\end{array}$ & $\begin{array}{c}81.3 \\
P\end{array}$ & NA & NA & $N D<0.038$ & 0.038 & $\begin{array}{c}F F>99.98 \\
S D A-F F>99.96 \\
\end{array}$ \\
\hline
\end{tabular}

NA - Not available. ND - Nondetect. NR - Not required. P-Pass. F - Fail. $x$ - Value invalid because of high background level.

$y$-Value considered an outlier. Note: A detailed discussion of QA/QC criteria is presented in Section 4.2.1. 
Table A-20. Total Vanadium

\begin{tabular}{|c|c|c|c|c|c|c|c|c|c|c|c|c|}
\hline \multirow{2}{*}{$\begin{array}{l}\text { Station/ } \\
\text { Plant } \\
\text { Name }\end{array}$} & \multirow{2}{*}{$\begin{array}{c}\text { Total } \\
\text { Stack } \\
\text { Conc., } \\
\mu \mathrm{g} / \mathrm{Nm}^{3}\end{array}$} & \multirow{2}{*}{$\begin{array}{l}\text { Total } \\
\text { Mean } \\
\text { Conc., } \\
\mu \mathrm{g} / \mathrm{Nm}^{3}\end{array}$} & \multirow[b]{2}{*}{$\begin{array}{l}\text { Standard } \\
\text { Deviation }\end{array}$} & \multirow{2}{*}{$\begin{array}{l}\text { Blank } \\
\text { Conc. } \\
\text { Range, } \\
\mu \mathrm{g} / \mathrm{Nm}^{3}\end{array}$} & \multicolumn{2}{|c|}{$\begin{array}{l}\text { M29 Internal Spike } \\
\text { Recovery, \% }\end{array}$} & \multirow{2}{*}{$\begin{array}{c}\text { M29 } \\
\text { Audit Spike } \\
\text { Recovery, } \\
\%\end{array}$} & \multirow[b]{2}{*}{$\begin{array}{c}\text { Above } \\
\text { DL }\end{array}$} & \multirow[b]{2}{*}{$\begin{array}{l}\text { Above } \\
\text { LLO }\end{array}$} & \multirow{2}{*}{$\begin{array}{l}\text { Emission } \\
\text { Factor, } \\
\text { Ib/10 } 12 \text { Btu }\end{array}$} & \multirow{2}{*}{$\begin{array}{l}\text { Emission } \\
\text { Factor } \\
95 \% \mathrm{Cl}\end{array}$} & \multirow{2}{*}{$\begin{array}{c}\text { Control } \\
\text { Device } \\
\text { Removal, } \\
\%\end{array}$} \\
\hline & & & & & Filter & Peroxide & & & & & & \\
\hline $\begin{array}{l}\text { Bailly } \\
\text { Station, } \\
\text { M29 }\end{array}$ & $\begin{array}{l}4.67 \\
3.61 \\
2.89\end{array}$ & 3.81 & 0.90 & $\mathbf{P}$ & $\begin{array}{c}106.4 \\
116.4 \\
P\end{array}$ & $\begin{array}{c}95.2 \\
92.3 \\
P\end{array}$ & NA & NA & NA & 2.8 & 1.6 & $\begin{array}{c}\text { ESP } \# 798.42 \\
\text { ESP } \# 899.83 \\
\text { AFGD } 76.9\end{array}$ \\
\hline $\begin{array}{c}\text { Baldwin } \\
\text { Power Station, } \\
\text { M29 } \\
\text { Non-Sootblowing }\end{array}$ & $\begin{array}{l}146 \\
150 \\
113\end{array}$ & 137 & 20 & $\mathbf{P}$ & NA & NA & NA & $\mathbf{p}$ & $P$ & 100 & 36 & ESP 92 \\
\hline $\begin{array}{c}\text { Baldwin } \\
\text { Power Station, } \\
\text { M29 } \\
\text { Sootblowing }\end{array}$ & $\begin{array}{l}168 \\
389 \\
335\end{array}$ & 298 & 115 & $P$ & NA & NA & NA & $P$ & $\mathrm{P}$ & 220 & 220. & ESP 90 \\
\hline $\begin{array}{c}\text { Boswell } \\
\text { Energy Center. } \\
\text { M29 }\end{array}$ & $\begin{array}{l}1.32 \\
1.68 \\
3.13\end{array}$ & 2.04 & 0.96 & $P$ & NA & NA & NA & $P$ & $F$ & 1.5 & 1.7 & $F F>99$ \\
\hline $\begin{array}{c}\text { Cardinal } \\
\text { Plant, } \\
\text { M29 } \\
\text { Non-Sootblowing }\end{array}$ & $\begin{array}{l}2.10 \\
2.31 \\
1.72 \\
2.05\end{array}$ & 2.045 & 0.244 & $P$ & $\begin{array}{c}77 \\
p\end{array}$ & $\begin{array}{c}70 \\
\mathrm{P}\end{array}$ & NA & NA & NA & 1.6 & 0.71 & \\
\hline $\begin{array}{l}\text { Cardinal } \\
\text { Plant, } \\
\text { M29 } \\
\text { Sootblowing }\end{array}$ & $\begin{array}{l}0.491 \\
1.31 \\
0.965\end{array}$ & 0.922 & 0.411 & $\mathbf{P}$ & $\begin{array}{c}77 \\
P\end{array}$ & $\begin{array}{c}70 \\
P\end{array}$ & NA & NA & NA & 0.72 & 0.89 & \\
\hline $\begin{array}{l}\text { Coal Creek } \\
\text { Station, } \\
\text { M29 }\end{array}$ & $\begin{array}{l}7.33 \\
5.51 \\
4.77\end{array}$ & 5.87 & 1.31 & $\begin{array}{c}N D<0.069 \\
N D<1.66 \\
p\end{array}$ & $\underset{P}{92-102}$ & $\underset{p}{97.5}$ & NA & $p$ & $F$ & 4.4 & 2.4 & $\begin{array}{c}\text { ESP\#1 } 99.6 \\
\text { Wet FGD } 9.6 \\
\text { Combined } 99.4\end{array}$ \\
\hline $\begin{array}{l}\text { Niles } \\
\text { Station, } \\
\text { M29 }\end{array}$ & $\begin{array}{l}3.74 \\
4.02 \\
4.88\end{array}$ & 4.21 & 0.59 & ND $<\underset{p}{0.114}$ & \multicolumn{2}{|c|}{$\underset{P}{86-114}$} & NA & P & $\mathbf{F}$ & 2.5 & 0.85 & ESP 99.56 \\
\hline $\begin{array}{c}\text { Niles/SNOX } \\
\text { Station, } \\
\text { M29 }\end{array}$ & $\begin{array}{l}N D<0.137 \\
N D<0.138 \\
N D<0.162\end{array}$ & $N D<0.146$ & 0.014 & ND $<\underset{F}{0.110}$ & \multicolumn{2}{|c|}{$\underset{P}{86-114}$} & NA & $\mathbf{F}$ & $F$ & ND $<0.11$ & 0.11 & $\begin{array}{c}\text { FF } 100 \\
\text { SNOX } 99.99\end{array}$ \\
\hline $\begin{array}{l}\text { Plant } \\
\text { Yates, } \\
\text { M29 }\end{array}$ & $\begin{array}{l}1.89 \\
1.97 \\
2.65\end{array}$ & 2.17 & 0.42 & $\frac{0.821}{F}$ & $\begin{array}{c}94 \\
\mathrm{P}\end{array}$ & $\begin{array}{c}107 \\
P\end{array}$ & NA & $p$ & $\mathrm{~F}$ & 2.1 & 1.0 & $\begin{array}{l}\text { ESP } 98.0 \\
\text { JBR } 96.0\end{array}$ \\
\hline $\begin{array}{l}\text { Springerville } \\
\text { Generating } \\
\text { Station, }\end{array}$ & $\begin{array}{r}1.0 \\
1.4 \\
2.17\end{array}$ & 1.5 & 0.6 & NA & $\begin{array}{c}90, \\
96 \\
p\end{array}$ & $\begin{array}{c}94 . \\
95 \\
\mathrm{P}\end{array}$ & NA & NA & NA & 1.0 & 1.0 & $\begin{array}{c}\text { FF } 99.98 \\
\text { SDA-FF } 99.96\end{array}$ \\
\hline
\end{tabular}

NA - Not available. ND - Nondetect. NR - Not required. P - Pass. F - Fail. $x$ - Value invalid because of high background level.

$y$-Value considered an outlier. Note: A detailed discussion of $Q A / Q C$ criteria is presented in Section 4.2.1. 
APPENDIX B

\section{ORGANIC DATA FOR STACK GAS SAMPLES}


Table B-1. Aldehyde Emissions from Niles, SNOX, and Coal Creek Station Stacks

\begin{tabular}{|c|c|c|c|c|}
\hline Species & $\begin{array}{c}\text { Reported Conc. } \\
\text { Mean } \pm \mathrm{SD} \\
\mu \mathrm{g} / \mathrm{m}^{3 \mathrm{a}}\end{array}$ & Data Quality & $\begin{array}{c}\text { Emission Factor, } \\
\mathrm{lb} / 10^{12} \mathrm{Btu}\end{array}$ & $95 \% \mathrm{Cl}$ \\
\hline \multicolumn{5}{|l|}{ Niles Station } \\
\hline Formaldehyde & $7 \pm 6$ & $\mathbf{P}$ & 4 & 9 \\
\hline Acetaldehyde & $150 \pm 130$ & $P$ & 89 & 180 \\
\hline Acrolein & $69 \pm 100$ & $\mathbf{P}$ & 41 & 150 \\
\hline Propionaldehyde & $42 \pm 36$ & $\mathbf{P}$ & 25 & 52 \\
\hline \multicolumn{5}{|l|}{ Niles Station/SNOX } \\
\hline Formaldehyde & $78 \pm 8$ & $P$ & 57 & 24 \\
\hline Acetaldehyde & $530 \pm 27$ & $P$ & 390 & 130 \\
\hline Acrolein & $11 \pm 2$ & $P$ & 8 & 3 \\
\hline Propionaldehyde & $19 \pm 14$ & $\mathbf{P}$ & 13 & 21 \\
\hline \multicolumn{5}{|l|}{ Coal Creek Station } \\
\hline Formaldehyde & $<3$ & FB & $-^{c}$ & $-^{c}$ \\
\hline Acetaldehyde & $88 \pm 13$ & $\mathrm{P}$ & 67 & 28 \\
\hline Acrolein & $<3$ & $P$ & $<2$ & $-^{c}$ \\
\hline Propionaldehyde & $16 \pm 5$ & $\mathrm{P}$ & 12 & 12 \\
\hline \multicolumn{5}{|c|}{$\begin{array}{l}\text { Means and standard deviations (SDs) based on three measurements. In cases where one or two values were } \\
\text { less than the detection limit, then one-half of the detection limit was used to calculate the mean and SD. } \\
\text { "P" indicates the results generally passed three indicators of data quality, and "F" indicates significant failure in } \\
\text { one of the indicators to the degree that the results should not be trusted; a listing of "B" indicates that some of } \\
\text { the blank values exceeded } 50 \% \text { of the measured values. } \\
\text { Emission factors not reported by the contractor because of high blank values. }\end{array}$} \\
\hline
\end{tabular}

Table B-2. Summary of VOC Emitted from Niles Station Stack

\begin{tabular}{|c|c|c|c|c|}
\hline Species & $\begin{array}{c}\text { Reported Conc. } \\
\text { Mean } \pm S D \\
\mu \mathrm{g} / \mathrm{m}^{3 \mathrm{a}}\end{array}$ & Data Quality & $\begin{array}{l}\text { Emission Factor, } \\
\mathrm{lb} / 10^{12} \mathrm{Btu}\end{array}$ & $95 \% \mathrm{Cl}$ \\
\hline Chloromethane & $9 \pm 7$ & $\mathrm{Q}$ & 5 & 10 \\
\hline Methylene Chloride & $34 \pm 17$ & $F, B$ & $-c$ & $-{ }^{c}$ \\
\hline Acetone & $42 \pm 27$ & $F, B$ & $-{ }^{c}$ & $-{ }^{c}$ \\
\hline Carbon Disulfide & $10 \pm 5$ & $\mathrm{Q}$ & 6 & 8 \\
\hline 2-Butanone & $9 \pm 8$ & $\mathrm{Q}$ & 5 & 11 \\
\hline Benzene & $13 \pm 4$ & $\mathbf{P}$ & 8 & 6 \\
\hline 4-Methyl-2-pentanone & $9 \pm 7$ & $\mathrm{O}$ & 5 & 11 \\
\hline Tetrachloroethane & $9 \pm 2$ & $\mathrm{O}$ & 3 & 3 \\
\hline Toluene & $8 \pm 5$ & $\mathrm{Q}$ & 4 & 7 \\
\hline 2-Hexanone & $13 \pm 16$ & $\mathrm{Q}$ & 8 & 23 \\
\hline \multicolumn{5}{|c|}{$\begin{array}{l}\text { Means and SDs based on three measurements. In cases where one or two values were less than the detection } \\
\text { limit, then one-half of the detection limit was used to calculate the mean and SD. } \\
\text { "P" indicates the results generally passed three indicators of data quality, and "F" indicates significant failure in } \\
\text { one of the indicators to the degree that the results should not be trusted; a listing of "B" indicates that some of } \\
\text { the blank values exceeded } 50 \% \text { of the measured values. "Q" indicates emission factors are highly questionable } \\
\text { since one or two out of three samples were below the detection limit or because insufficient information was } \\
\text { available on either blank, spike, or audit tests. } \\
\text { Emission factors not reported by the contractor because of high blank values. }\end{array}$} \\
\hline
\end{tabular}


Table B-3. Summary of VOC Emitted from Plant Yates Stack

\begin{tabular}{|c|c|c|c|c|}
\hline Species & $\begin{array}{c}\text { Reported Conc. } \\
\text { Mean } \pm \mathrm{SD} \\
\mu \mathrm{g} / \mathrm{Nm}^{3 a}\end{array}$ & Data Quality ${ }^{b}$ & $\begin{array}{c}\text { Emission Factor, } \\
\mathrm{lb} / 10^{12} \mathrm{Btu}\end{array}$ & $95 \% \mathrm{Cl}$ \\
\hline 1,1,1-Trichloroethane & $1 \pm 0.3$ & FA & $-^{c}$ & $-^{c}$ \\
\hline Acetone & $4 \pm 3$ & FB & $-{ }^{c}$ & $-^{c}$ \\
\hline Benzene & $1 \pm 0.2$ & $\mathbf{P}$ & 1 & 0.3 \\
\hline Carbon Disulfide & $2 \pm 1$ & $\mathrm{Q}$ & 2 & 1 \\
\hline Chloromethane & $6 \pm 5$ & FB & $-{ }^{c}$ & $-c$ \\
\hline Methylene Chloride & $130 \pm 110$ & $F B, F A$ & $-^{c}$ & $-^{c}$ \\
\hline Tetrachloroethane & $2 \pm 1$ & FB & $-{ }^{c}$ & $-^{c}$ \\
\hline Toluene & $2 \pm 0.4$ & FA & 2 & 1 \\
\hline Trichlorofluoromethane & $1 \pm 1$ & FA & $-^{c}$ & $-^{c}$ \\
\hline \multicolumn{5}{|c|}{$\begin{array}{l}\text { Means and SDs based on three measurements. In cases where one or two values were less than the detection } \\
\text { limit, then one-half of the detection limit was used to calculate the mean and SD. } \\
\text { "P" indicates the results generally passed three indicators of data quality, and "F" indicates significant failure in } \\
\text { one of the indicators to the degree that the results should not be trusted; a listing of "B" indicates that some of } \\
\text { the blank values exceeded } 50 \% \text { of the measured values. "A" indicate that the contractor generally failed to } \\
\text { meet method recovery criteria for audit samples. "Q" indicates emission factors are highly questionable since } \\
\text { one or two out of three samples were below the detection limit or because insufficient information was available } \\
\text { on either blank, spike, or audit tests. }\end{array}$} \\
\hline Species & $\begin{array}{c}\text { Reported Conc. } \\
\text { Mean } \pm \mathrm{SD} \\
\mu \mathrm{g} / \mathrm{Nm}^{3 \mathrm{a}}\end{array}$ & Data Quality ${ }^{b}$ & $\begin{array}{c}\text { Emission Factor, } \\
\mathrm{lb} / 10^{12} \mathrm{Btu}\end{array}$ & $95 \% \mathrm{Cl}$ \\
\hline Chloromethane & $300 \pm 260$ & $\mathbf{P}$ & 220 & 470 \\
\hline Bromomethane & $16 \pm 5$ & $Q$ & 10 & 9 \\
\hline Chloroethane & $<4.7^{c}$ & 0 & 3 & 2 \\
\hline Methylene Chloride & $36 \pm 22$ & FB & $-^{d}$ & $-{ }^{d}$ \\
\hline Acetone & $63 \pm 29$ & FB & $-{ }^{d}$ & $-^{d}$ \\
\hline Carbon Disulfide & $<4.6^{\mathrm{c}}$ & Q & $<5.4$ & 5 \\
\hline 1,1,2-Trichloroethane & $<13^{\mathrm{c}}$ & $\mathrm{Q}$ & $<4.9$ & 10 \\
\hline Benzene & $8 \pm 3$ & $P$ & 6 & 5 \\
\hline 2-Hexanone & $26 \pm 28$ & 0 & 19 & 51 \\
\hline Toluene & $6.1^{\mathrm{C}}$ & Q & 4 & 5 \\
\hline
\end{tabular}

a Means and SDs based on three measurements. In cases where one or two values were less than the detection limit, then one-half of the detection limit was used to calculate the mean and SD.

b " $P$ " indicates the results generally passed three indicators of data quality, and " $F$ " indicates significant failure in one of the indicators to the degree that the results should not be trusted; a listing of "B" indicates that some of the blank values exceeded $50 \%$ of the measured values. " $Q$ " indicates emission factors are highly questionable since one or two out of three samples were below the detection limit or because insufficient information was available on either blank, spike, or audit tests.

c Detection limits varied between samples. Compound was detected in one or two samples, but at concentrations lower than the detection limit for the other sample(s). Values are the mean (for two detected values) or the single value (for one detection value).

d Emission factors not reported by the contractor because of high blank values. 
Table B-5. Summary of VOC Emitted from Coal Creek Station Stack

\begin{tabular}{lcccc}
\hline Species & $\begin{array}{c}\text { Reported Conc. } \\
\text { Mean } \pm \mathrm{SD}, \\
\mu \mathrm{g} / \mathrm{Nm}^{3 \mathrm{a}}\end{array}$ & $\begin{array}{c}\text { Data Quality } \\
\text { D }\end{array}$ & $\begin{array}{c}\text { Emission Factor, } \\
\mathrm{lb} / 10^{12} \mathrm{Btu}\end{array}$ & $95 \% \mathrm{Cl}$ \\
\hline Chloromethane & $140 \pm 40$ & $\mathrm{P}$ & 110 & 70 \\
Bromomethane & $9 \pm 3$ & $\mathrm{Q}$ & 4 & 5 \\
Methylene Chloride & $490 \pm 280$ & $\mathrm{FB}$ & $-{ }^{\mathrm{d}}$ & $-{ }^{\mathrm{d}}$ \\
Acetone & $34 \pm 7$ & $\mathrm{FB}$ & $-{ }^{\mathrm{d}}$ & $-{ }^{\mathrm{d}}$ \\
Carbon Disulfide & $<5^{\mathrm{c}}$ & $\mathrm{Q}$ & 3 & 1 \\
1, 2-Dichloroethane & $<4^{\mathrm{c}}$ & $\mathrm{Q}$ & 3 & 1 \\
2-Butanone & $13 \pm 6$ & $\mathrm{P}$ & 10 & 10 \\
Benzene & $53 \pm 19$ & $\mathrm{P}$ & 40 & 40 \\
Bromoform & $<4^{\mathrm{c}}$ & $\mathrm{Q}$ & 3 & 12 \\
4-Methyl-2-pentanone & $9 \pm 7$ & $\mathrm{Q}$ & 6 & 10 \\
2-Hexanone & $15 \pm 16$ & $\mathrm{P}$ & 10 & 30 \\
Toluene & $31 \pm 10$ & $\mathrm{P}$ & 20 & 20 \\
Chlorobenzene & $<4^{\mathrm{c}}$ & $\mathrm{O}$ & 3 & 1 \\
Styrene & $<5^{\mathrm{c}}$ & $\mathrm{Q}$ & 3 & 1 \\
Xylenes & $<5^{\mathrm{c}}$ & $\mathrm{Q}$ & 4 & 1 \\
\hline
\end{tabular}

a Means and SDs based on three measurements. In cases where one or two values were less than the detection limit, then one-half of the detection limit was used to calculate the mean and SD.

b " $P$ " indicates the results generally passed three indicators of data quality, and " $F$ " indicates significant failure in one of the indicators to the degree that the results should not be trusted; a listing of "B" indicates that some of the blank values exceeded $50 \%$ of the measured values. " $Q$ " indicates emission factors are highly questionable since one or two out of three samples were below the detection limit or because insufficient information was available on either blank, spike, or audit tests.

c Detection limits varied between samples. Compound was detected in one or two samples, but at concentrations lower than the detection limit for the other sample(s). Values are the mean (for two detected values) or the single value (for one detection value).

d Emission factors not reported by contractor because of high blank values.

Table B-6. Summary of VOC Emitted from Springerville Station Stack

\begin{tabular}{lcccc}
\hline & $\begin{array}{c}\text { Reported Conc. } \\
\text { Mean } \pm \mathrm{SD}, \\
\mu \mathrm{g} / \mathrm{Nm}^{3 \mathrm{a}}\end{array}$ & Data Quality & $\begin{array}{c}\text { Emission Factor, } \\
\mathrm{lb} / 10^{12} \mathrm{Btu}\end{array}$ & $95 \% \mathrm{Cl}$ \\
\hline Benzies & $1.4 \pm 0.2$ & $\mathrm{Q}$ & 1 & 0.4 \\
Toluene & $0.8 \pm 0.3$ & $\mathrm{Q}$ & 1 & 0.4 \\
$m, p$-Xylene & $0.03 \pm 0.1$ & $\mathrm{Q}$ & 0.02 & 0.1 \\
\hline
\end{tabular}

a Means and SDs based on three measurements. In cases where one or two values were less than the detection limit, then one-half of the detection limit was used to calculate the mean and SD.

b " $O$ " indicates emission factors are highly questionable since one or two out of three samples were below the detection limit or because insufficient information was available on either blank, spike, or audit tests. 
Table B-7. Summary of VOC Emitted from Cardinal Station Stack

\begin{tabular}{lcccr}
\hline Species & $\begin{array}{c}\text { Reported Conc. } \\
\text { Mean } \pm \text { SD, } \\
\mu \mathrm{g} / \mathrm{Nm}^{3 \mathrm{a}}\end{array}$ & $\begin{array}{c}\text { Data Quality } \\
\text { Acetone }\end{array}$ & $\begin{array}{c}\text { Emission Factor, } \\
\mathrm{lb} / 10^{12} \mathrm{Btu}\end{array}$ & $95 \% \mathrm{Cl}$ \\
\hline Benzene & $53 \pm 110$ & $\mathrm{P}$ & 42 & 150 \\
Bromomethane & $4 \pm 3$ & $\mathrm{P}$ & 3 & 48 \\
Chloroform & $19 \pm 33$ & $\mathrm{Q}$ & 15 & 110 \\
Chloromethane & $4 \pm 5$ & $\mathrm{Q}$ & 3 & 83 \\
$n$-Hexane & $8 \pm 2$ & $\mathrm{Q}$ & 6 & 250 \\
lodomethane & $8 \pm 2$ & $\mathrm{Q}$ & 7 & 24 \\
$m, p$-Xylene & $12 \pm 9$ & $\mathrm{Q}$ & 10 & 55 \\
Methyl Hydrazine & $4 \pm 3$ & $\mathrm{P}$ & 3 & 120 \\
Methyl tert-Butyl Ether & $8 \pm 2$ & FA & 7 & 150 \\
Toluene & $2 \pm 0.4$ & $\mathrm{O}$ & 1 & 26 \\
Trichlorofluoromethane & $7 \pm 4$ & FB & 5 & 120 \\
\hline
\end{tabular}

a Means and SDs based on three measurements. In cases where one or two values were less than the detection limit, then one-half of the detection limit was used to calculate the mean and SD. SDs were not available.

- " $P$ " indicates the results generally passed three indicators of data quality, and " $F$ " indicates significant failure in one of the indicators to the degree that the results should not be trusted; a listing of "B" indicates that some of the blank values exceeded $50 \%$ of the measured values. " $A$ " indicates that the contractor failed to meet method recovery criteria for audit samples. " $Q$ " indicates emission factors are highly questionable since one or two out of three samples were below the detection limit or because insufficient information was available on either blank, spike, or audit tests. 
Table B-8. Summary of VOC Emitted from Boswell Energy Center Stack

\begin{tabular}{|c|c|c|c|c|c|c|}
\hline \multirow{2}{*}{$\begin{array}{l}\text { Species } \\
\text { Chloroethane }\end{array}$} & \multicolumn{3}{|c|}{$\begin{array}{c}\text { Reported Conc. } \\
\text { Mean } \pm \mathrm{SD} \\
\mu \mathrm{g} / \mathrm{Nm}^{3 \mathrm{a}} \\
\end{array}$} & \multirow{2}{*}{$\frac{\begin{array}{c}\text { Data } \\
\text { Quality }^{\mathrm{b}}\end{array}}{\mathrm{a}}$} & \multirow{2}{*}{$\begin{array}{c}\begin{array}{c}\text { Emission Factor, } \\
\mathrm{lb} / 10^{12} \mathrm{Btu}\end{array} \\
3\end{array}$} & \multirow{2}{*}{$\frac{95 \% \mathrm{Cl}}{6}$} \\
\hline & 3 & \pm & 3 & & & \\
\hline Trichlorofluoromethane & 2 & \pm & 3 & $P$ & 2 & 3 \\
\hline Carbon Disulfide & 23 & \pm & 21 & $P$ & 18 & 41 \\
\hline Acetone & 110 & \pm & 13 & FB & 84 & 23 \\
\hline Methylene Chloride & 14 & \pm & 3 & FB & 11 & 6 \\
\hline$n$-Hexane & 2 & \pm & 1 & $P$ & 2 & 3 \\
\hline Vinyl Acetate & 1 & \pm & 1 & $Q$ & 0.4 & 2 \\
\hline 2-Butanone & 21 & \pm & 5 & $P$ & 16 & 9 \\
\hline Benzene & 130 & \pm & 93 & $\dot{\mathbf{P}}$ & 100 & 180 \\
\hline Methyl Methacrylate & 2 & \pm & 3 & Q & 1 & 5 \\
\hline Ethylene Dibromide & 0.1 & \pm & 0.1 & 0 & 0.1 & 0.2 \\
\hline Toluene & 7 & \pm & 1 & FB & 6 & 3 \\
\hline Tetrachloroethane (PCE) & 1 & \pm & 1 & 0 & 1 & 2 \\
\hline Chlorobenzene & 0.2 & \pm & 0.3 & $\mathrm{Q}$ & 0.2 & 1 \\
\hline Ethylbenzene & 1 & \pm & 0.4 & $P$ & 0.4 & 1 \\
\hline$m-/ p$-Xylene & 3 & \pm & 1 & $P$ & 2 & 2 \\
\hline$o$-Xylene & 0.4 & \pm & 0.2 & $P$ & 0.3 & 0.4 \\
\hline Styrene & 2 & \pm & 2 & $P$ & 2 & 5 \\
\hline Cumene & 0.4 & \pm & 0.2 & $P$ & 0.3 & 0.3 \\
\hline
\end{tabular}

a Mean and SD is based on nine measurements, but represents mean of mean of 3 days leach with three experiments). In cases where one or two values (or less) were less than the detection limit, then one-half of the detection limit was used to calculate the mean and SD.

b " $P$ " indicates the results generally passed three indicators of data quality, and " $F$ " indicates significant failure in one of the indicators to the degree that the results should not be trusted; a listing of " $B$ " indicates that some of the blank values exceeded $50 \%$ of the measured values. " $Q$ " indicates emission factors are highly questionable since one or two out of three samples were below the detection limit or because insufficient information was available on either blank, spike, or audit tests. 
Table B-9. Summary of VOC Emitted from Baldwin Power Station Stack

\begin{tabular}{|c|c|c|c|c|}
\hline Species & $\begin{array}{c}\text { Reported Conc. } \\
\text { Mean } \pm \mathrm{SD} \\
\mu \mathrm{g} / \mathrm{Nm}^{3 \mathrm{a}}\end{array}$ & $\begin{array}{c}\text { Data } \\
\text { Quality } \\
\end{array}$ & $\begin{array}{c}\text { Emission Factor, } \\
\mathrm{lb} / 10^{12} \mathrm{Btu}\end{array}$ & $95 \% \mathrm{Cl}$ \\
\hline Benzene & $170 \pm 220$ & $\mathbf{P}$ & 120 & 400 \\
\hline Methylene Chloride & $25 \pm 8$ & $\mathrm{~F}, \mathrm{~B}$ & 18 & 14 \\
\hline$m-/ p$-Xylene & $2 \pm 0.2$ & $P$ & 1 & 1 \\
\hline$n$-Hexane & $0.2 \pm 0.1$ & Q & 0.2 & 0.2 \\
\hline$o$-Xylene & $1 \pm 0.1$ & $\mathbf{P}$ & 1 & 0.3 \\
\hline Toluene & $3 \pm 1$ & $F, B$ & 2 & 2 \\
\hline Trichlorofluoromethane & $4 \pm 1$ & $\mathbf{P}$ & 3 & 2 \\
\hline Carbon Disulfide $^{c}$ & $0.2 \pm 0.2$ & $\mathrm{Q}$ & 0.14 & 0.3 \\
\hline Styrene & $0.3 \pm 0.2$ & $\mathbf{P}$ & 0.2 & 0.4 \\
\hline Bromomethane (methyl bromide) & $1 \pm 2$ & Q & 1 & 4 \\
\hline Ethylbenzene & $0.2 \pm 0.2$ & 0 & 0.1 & 0.4 \\
\hline lodomethane $^{c}$ & $1 \pm 1$ & Q & 0.4 & 2 \\
\hline
\end{tabular}

a Means and SDs are based on nine measurements but represent mean of mean of 3 days (each with three experiments). In cases where one or two values (or less) were less than the detection limit, then one-half of the detection limit was used to calculate the mean and SD.

- " $P$ " indicates the results generally passed three indicators of data quality, and " $F$ " indicates significant failure in one of the indicators to the degree that the results should not be trusted; a listing of "B" indicates that some of the blank values exceeded $50 \%$ of the measured values. " $Q$ " indicates emission factors are highly questionable since one or two out of three samples were below the detection limit or because insufficient information was available on either blank, spike, or audit tests.

c Only detected in one to three samples out of nine. 
Table B-10. Summary of SVOC Emitted from Baldwin Power Station Stack

\section{Reported Conc.}

Mean \pm SD,

Species

$\mu \mathrm{g} / \mathrm{Nm}^{3 a}$

Data Quality

Emission Factor,

Phenol

$1^{\mathrm{c}}$

Q

$\mathrm{Ib} / 10^{12}$ Btu

$95 \% \mathrm{Cl}$

2-Methylphenol

$$
2 \pm 3
$$

Q

$<1$

0.7

Acetophenone

3/4-Methylphenol

$$
2 \pm 0.1
$$

o

1.8

3.8

Isophorone

$$
1 \pm 2
$$

Q

1.2

0.1

Naphthalene

Di-n-butyl Phthalate

$40 \pm 30$

FB

0.8

1.7

Bis(2-ethylhexy)phthalate

$$
2^{\mathrm{c}}
$$

FB

26

59

2-Methylnaphthalene

$4^{c}$

FB

$<3$

2.9

2-Chloronaphthalene

$$
6 \pm 4
$$

Q

$<3$

1.7

Acenaphthylene

$0.03^{\mathrm{c}}$

FB

4.6

2.4

$$
0.001 \pm 0.0002
$$

FB

$<0.034$

0.5

Fluorene

$$
0.04 \pm 0.03
$$$$
\text { P }
$$

0.0004

0.02

Phenanthrene

$$
0.002^{c}
$$

FB

$$
0.03
$$

0.0004

$$
0.1 \pm 0.03
$$

$P$

$<0.01$

0.06

Anthracene

$$
0.002^{c}
$$

FB

$<0.01$

0.01

Fluoranthene

$0.02 \pm 0.01$

FB

$$
0.1
$$

0.01

Pyrene

$$
0.01^{\mathrm{c}}
$$

FB

$<0.003$

0.05

Chrysene

$0.001^{\mathrm{c}}$

FB

$$
0.02
$$

0.003

Benzo[k]fluoranthene

$$
0.0002^{c}
$$

FB

$<0.003$

0.03

a Means and SDs are based on three measurements. In cases where one or two values were less than the detection limit, then one-half of the detection limit was used to calculate the mean and SD.

b " $P$ " indicates the results generally passed three indicators of data quality, and " $F$ " indicates significant failure in one of the indicators to the degree that the results should not be trusted; a listing of " $B$ " indicates that some of the blank values exceeded $50 \%$ of the measured values. " $\mathrm{O}$ " indicates emission factors are highly questionable since one or two out of three samples were below the detection limit or because insufficient information was available on either blank, spike, or audit tests.

c Detection limits varied between samples. Compound was detected in one or two samples, but at concentrations lower than the detection limit for the other sample(s). Values are the mean (for two detected values) or the single value (for one detection value). 
Table B-11. Summary of SVOC Emitted from SNOX Process Stack

\begin{tabular}{|c|c|c|c|c|}
\hline Species & $\begin{array}{c}\text { Reported Conc. } \\
\text { Mean } \pm \mathrm{SD} \\
\mu \mathrm{g} / \mathrm{Nm}^{3 \mathrm{a}}\end{array}$ & $\begin{array}{c}\text { Data } \\
\text { Quality }^{\mathrm{b}} \\
\end{array}$ & $\begin{array}{c}\text { Emission Factor, } \\
\mathrm{lb} / 10^{12} \mathrm{Btu}\end{array}$ & $95 \% \mathrm{Cl}$ \\
\hline Benzyl Chloride & $0.04 \pm 0.1$ & 0 & 0.03 & 0.1 \\
\hline Acetophenone & $0.42 \pm 0.3$ & $P$ & 0.3 & 0.44 \\
\hline Naphthalene & $0.08 \pm 0.03$ & $\mathrm{FB}$ & 0.1 & 0.1 \\
\hline 2-Methylnaphthalene & $0.03 \pm 0.02$ & $P$ & 0.02 & 0.044 \\
\hline 1-Methylnaphthalene & $0.02 \pm 0.01$ & $\mathbf{P}$ & 0.011 & 0.023 \\
\hline Biphenyl & $0.01 \pm 0.01$ & FB & 0.01 & 0.02 \\
\hline Acenaphthylene & $0.01 \pm 0.004$ & $P$ & 0.0042 & 0.01 \\
\hline Acenaphthene & $0.01 \pm 0.004$ & $P$ & 0.01 & 0.01 \\
\hline Dibenzofuran & $0.02 \pm 0.01$ & $P$ & 0.013 & 0.01 \\
\hline 2,4-Dinitrotoluene & $<0.01 \pm 0.001$ & $Q$ & 0.004 & 0.001 \\
\hline Fluorene & $<0.002 \pm 0.0004$ & FB & 0.001 & 0.001 \\
\hline Pentachlorophenol & $<0.01 \pm 0.002$ & $\mathrm{Q}$ & 0.0032 & 0.0031 \\
\hline Phenanthrene & $0.03 \pm 0.02$ & FB & 0.024 & 0.03 \\
\hline Anthracene & $0.01 \pm 0.003$ & $P$ & 0.004 & 0.01 \\
\hline Fluoranthene & $0.01 \pm 0.004$ & FB & 0.007 & 0.01 \\
\hline Pyrene & $0.002 \pm 0.002$ & FB & 0.0012 & 0.003 \\
\hline Benz[a]anthracene & $0.003 \pm 0.0003$ & $\mathbf{P}$ & 0.0021 & 0.001 \\
\hline Chrysene & $0.003 \pm 0.002$ & $\mathbf{P}$ & 0.0021 & 0.003 \\
\hline Benzo[ $b+k]$ fluoranthene & $0.01 \pm 0.001$ & $P$ & 0.004 & 0.0021 \\
\hline Benzo[e]pyrene & $<0.002 \pm 0.001$ & $\mathrm{Q}$ & 0.0011 & 0.002 \\
\hline Benzo[a]pyrene & $<0.002 \pm 0.0004$ & 0 & 0.001 & 0.001 \\
\hline Indenol $1,2,3-c, d]$ pyrene & $<0.002 \pm 0.001$ & $\mathrm{O}$ & 0.001 & 0.001 \\
\hline Dibenzo[ah]anthracene & $<0.002 \pm 0.0002$ & $\mathrm{O}$ & 0.001 & 0.0004 \\
\hline Benzo[ghi]perylene & $<0.002 \pm 0.0004$ & $\mathrm{Q}$ & 0.001 & 0.001 \\
\hline
\end{tabular}

a Means and SDs are based on three measurements. In cases where one or two values were less than the detection limit, then one-half of the detection limit was used to calculate the mean and SD.

b " $P$ " indicates the results generally passed three indicators of data quality, and " $F$ " indicates significant failure in one of the indicators to the degree that the results should not be trusted; a listing of " $B$ " indicates that some of the blank values exceeded $50 \%$ of the measured values. " $Q$ " indicates emission factors are highly questionable since one or two out of three samples were below the detection limit or because insufficient information was available on either blank, spike, or audit tests. 
Table B-12. Summary of SVOC Emitted from Niles Station Stack

\begin{tabular}{|c|c|c|c|c|}
\hline Species & $\begin{array}{c}\text { Reported Conc. } \\
\text { Mean } \pm \text { SD } \\
\left(\mu \mathrm{g} / \mathrm{Nm}^{3}\right)^{2}\end{array}$ & $\begin{array}{c}\text { Data } \\
\text { Quality } \\
\end{array}$ & $\begin{array}{c}\text { Emission Factor } \\
\left(\mathrm{lb} / 10^{12} \mathrm{Btu}\right)\end{array}$ & $95 \% \mathrm{Cl}$ \\
\hline Acetophenone & $1.1 \pm 0.5$ & $P$ & 0.64 & 0.74 \\
\hline Naphthalene & $0.37 \pm 0.2$ & $P$ & 0.22 & 0.25 \\
\hline 2-Chloroacetophenone & $0.49 \pm 0.4$ & 0 & 0.3 & 0.52 \\
\hline 2-Methylnaphthalene & $0.06 \pm 0.06$ & $P$ & 0.04 & 0.09 \\
\hline 1-Methylnaphthalene & $0.03 \pm 0.03$ & $P$ & 0.02 & 0.04 \\
\hline Biphenyl & $0.21 \pm 0.25$ & $P$ & 0.13 & 0.36 \\
\hline Acenaphthylene & $<0.01 \pm 0.02$ & 0 & 0.01 & 0.02 \\
\hline 2,6-Dinitrotoluene & $0.93 \pm 0.18$ & $P$ & 0.6 & 0.24 \\
\hline Acenaphthene & $0.05 \pm 0.06$ & $P$ & 0.03 & 0.08 \\
\hline Dibenzofuran & $0.11 \pm 0.09$ & $P$ & 0.1 & 0.13 \\
\hline 2,4-Dinitrotoluene & $<0.03 \pm 0.02$ & $\mathrm{Q}$ & 0.02 & 0.03 \\
\hline Fluorene & $0.1 \pm 0.06$ & $P$ & 0.03 & 0.09 \\
\hline Phenanthrene & $0.13 \pm 0.12$ & $P$ & 0.1 & 0.17 \\
\hline Anthracene & $0.04 \pm 0.05$ & $P$ & 0.02 & 0.07 \\
\hline Fluoranthene & $0.05 \pm 0.03$ & $P$ & 0.03 & 0.05 \\
\hline Pyrene & $0.02 \pm 0.02$ & $P$ & 0.01 & 0.03 \\
\hline Benz[a]anthracene & $0.01 \pm 0.01$ & $\mathrm{Q}$ & 0.004 & 0.01 \\
\hline Chrysene & $0.02 \pm 0.01$ & $P$ & 0.01 & 0.02 \\
\hline Benzo $[b+k]$ fluoranthene & $<0.01 \pm 0.02$ & 0 & 0.01 & 0.02 \\
\hline Benzo[e]pyrene & $<0.01 \pm 0.004$ & Q & 0.002 & 0.01 \\
\hline
\end{tabular}

a Means and SDs are based on three measurements. In cases where one or two values were less than the detection limit, then one-half of the detection limit was used to calculate the mean and SD.

- " $P$ " indicates the results generally passed three indicators of data quality. " $Q$ " indicates emission factors are highly questionable since one or two out of three samples were below the detection limit or because insufficient information was available on either blank, spike, or audit tests. 
Table B-13. Summary of SVOC Emitted from Boswell Energy Center Stack

\begin{tabular}{|c|c|c|c|c|}
\hline Species & $\begin{array}{c}\text { Reported Conc. } \\
\text { Mean } \pm \mathrm{SD} \\
\mu \mathrm{g} / \mathrm{Nm}^{3 \mathrm{a}}\end{array}$ & Data Quality ${ }^{6}$ & $\begin{array}{c}\text { Emission Factor, } \\
\mathrm{lb} / 10^{12} \mathrm{Btu}\end{array}$ & $95 \% \mathrm{Cl}$ \\
\hline Phenol & $0.6 \pm 0.32$ & $Q$ & 0.43 & 0.4 \\
\hline 2-Methylphenol & $1.3 \pm 1.13$ & 0 & 1.0 & 1.9 \\
\hline Acetophenone & $0.92 \pm 0.1$ & $\mathrm{FB}$ & 0.71 & 0.13 \\
\hline 3/4-Methylphenol & $0.9 \pm 0.8$ & $\mathrm{Q}$ & 0.65 & 1.4 \\
\hline Naphthalene & $0.3 \pm 0.44$ & FB & 0.95 & 0.3 \\
\hline 2-Methylnapththalene & $0.04 \pm 0.01$ & FB & 0.03 & 0.02 \\
\hline 2-Chloronaphthalene & $0.001 \pm 0.0003$ & FB & 0.001 & 0.001 \\
\hline Acenaphthylene & $0.01 \pm 0.01$ & FB & 0.01 & 0.01 \\
\hline Acenaphthene & $0.1 \pm 0.1$ & FB & 0.04 & 0.17 \\
\hline Fluorene & $0.01 \pm 0.02$ & FB & 0.01 & 0.03 \\
\hline Phenanthrene & $0.3 \pm 0.14$ & $\mathbf{P}$ & 0.21 & 0.26 \\
\hline Anthracene & $0.01 \pm 0.013$ & $\mathbf{P}$ & 0.01 & 0.03 \\
\hline Fluoranthene & $0.11 \pm 0.11$ & $\mathbf{P}$ & 0.08 & 0.21 \\
\hline Pyrene & $0.1 \pm 0.1$ & $P$ & 0.04 & 0.13 \\
\hline Benzo[a]anthracene & $0.01 \pm 0.01$ & $\mathbf{P}$ & 0.01 & 0.04 \\
\hline Chrysene & $0.02 \pm 0.02$ & $\mathbf{P}$ & 0.012 & 0.031 \\
\hline Benzo[b]fluranthene & $0.004 \pm 0.004$ & FB & 0.003 & 0.01 \\
\hline Benzo[k]fluoranthene & $0.0004 \pm 0.001$ & FB & 0.0003 & 0.001 \\
\hline Benzo[e]pyrene & $0.003 \pm 0.002$ & FB & 0.002 & 0.003 \\
\hline Benzo[a]pyrene & $0.0002 \pm 0.0001$ & FB & $<0.0002$ & 0.0001 \\
\hline Perylene & $0.102 \pm 0.2$ & FB & 0.1 & 0.34 \\
\hline Indeno $[1,2,3-c d]$ pyrene & $0.0001 \pm 0.0001$ & FB & $<0.0003$ & 0.0004 \\
\hline Dibenzo[ah]anthracene & $0.0001 \pm 0.00003$ & FB & $<0.0001$ & 0.0001 \\
\hline
\end{tabular}

Means and SDs are based on three measurements. In cases where one or two values were less than the detection limit, then one-half of the detection limit was used to calculate the mean and SD.

b " $P$ " indicates the results generally passed three indicators of data quality. " $F$ " indicates significant failure in one of the indicators to the degree that the results should not be trusted. A listing of " $B$ " indicates that some of the blank values exceeded $50 \%$ of the measured values. " $Q$ " indicates emission factors are highly questionable since one or two out of three samples were below the detection limit or because insufficient information was available on either blank, spike, or audit tests. 
Table B-14. Summary of SVOC Emitted from Plant Yates Stack

\begin{tabular}{|c|c|c|c|c|}
\hline Species & $\begin{array}{c}\text { Reported Conc. } \\
\text { Mean } \pm \mathrm{SD} \\
\mu \mathrm{g} / \mathrm{Nm}^{3 \mathrm{a}}\end{array}$ & Data Quality ${ }^{\mathrm{b}}$ & $\begin{array}{c}\text { Emission Factor, } \\
\mathrm{lb} / 10^{12} \mathrm{Btu}\end{array}$ & $95 \% \mathrm{Cl}$ \\
\hline 2-Methylphenol & $3 \pm 2$ & $\mathbf{P}$ & 3 & 4 \\
\hline 4-Methylphenol & $1 \pm 0.7$ & $\mathrm{Q}$ & 1 & 2 \\
\hline Acetophenone & $3 \pm 0.3$ & 0 & 3 & 1 \\
\hline Benzoic Acid & $120 \pm 2$ & $\mathrm{Q}$ & 120 & 7 \\
\hline Benzyl Alcohol & $3 \pm 5$ & $\mathbf{Q}$ & 3 & 12 \\
\hline Butylbenzylphthalate & $0.3 \pm 0.1$ & $Q$ & $-^{c}$ & $-^{c}$ \\
\hline Dibutylphthalate & $0.2 \pm 0.1$ & $Q$ & $-^{c}$ & $-^{c}$ \\
\hline Diethylphthalate & $0.2 \pm 0.1$ & Q & $-^{c}$ & $-^{c}$ \\
\hline Dimethylphthalate & $0.2 \pm 0.2$ & $\mathrm{O}$ & $-{ }^{c}$ & $-c$ \\
\hline Bis(2-ethylhexy 1 )phthalate & $1.4 \pm 1$ & $\mathrm{O}$ & $-^{c}$ & $-{ }^{c}$ \\
\hline Naphthalene & $2 \pm 0.4$ & $P$ & 2 & 1.0 \\
\hline Phenol & $9 \pm 4$ & $\mathbf{P}$ & 9 & 9 \\
\hline
\end{tabular}

Means and SDs based on three measurements. In cases where one or two values were less than the detection limit, then one-half of the detection limit was used to calculate the mean and SD.

b "P" indicates the results generally passed three indicators of data quality. " $Q$ " indicates emission factors are highly questionable since one or two out of three samples were below the detection limit or because insufficient information was available on either blank, spike, or audit tests.

c Not reported by the contractor. 
Table B-15. Summary of SVOC Emitted from Coal Creek Station Stack

\begin{tabular}{|c|c|c|c|c|}
\hline Species & $\begin{array}{l}\text { Reported Conc. } \\
\text { Mean } \pm \mathrm{SD} \\
\mu \mathrm{g} / \mathrm{Nm}^{3 \mathrm{a}}\end{array}$ & $\begin{array}{c}\text { Data } \\
\text { Quality }\end{array}$ & $\begin{array}{c}\text { Emission Factor, } \\
\mathrm{Ib} / 10^{12} \mathrm{Btu}\end{array}$ & $95 \% \mathrm{Cl}$ \\
\hline Benzyl Chloride & $0.01 \pm 0.01$ & $F B$ & 0.01 & 0.02 \\
\hline Acetophenone & $0.72 \pm 0.4$ & FB & 0.54 & 0.7 \\
\hline Naphthalene & $0.34 \pm 0.1$ & FB & 0.25 & 0.2 \\
\hline 2-Chloroacetophenone & $0.2 \pm 0.04$ & FB & 0.13 & 0.06 \\
\hline 2-MethyInaphthalene & $0.1 \pm 0.01$ & FB & 0.04 & 0.02 \\
\hline 1-MethyInaphthalene & $0.02 \pm 0.003$ & FB & 0.02 & 0.01 \\
\hline Biphenyl & $0.03 \pm 0.003$ & FB & 0.02 & 0.01 \\
\hline Acenaphthylene & $0.01 \pm 0.011$ & $\mathrm{FB}$ & 0.01 & 0.02 \\
\hline Acenaphthene & $0.02 \pm 0.012$ & FB & 0.02 & 0.02 \\
\hline Dibenzofuran & $0.07 \pm 0.013$ & FB & 0.05 & 0.03 \\
\hline 2,4-Dinitrotoluene & $0.01 \pm 0.01$ & $\mathrm{FB}$ & 0.01 & 0.01 \\
\hline Fluorene & $0.1 \pm 0.02$ & $\mathrm{FB}$ & 0.04 & 0.04 \\
\hline Phenanthrene & $0.40 \pm 0.3$ & FB & 0.31 & 0.6 \\
\hline Anthracene & $0.02 \pm 0.003$ & FB & 0.02 & 0.01 \\
\hline Fluoranthene & $0.1 \pm 0.02$ & $F B$ & 0.04 & 0.04 \\
\hline Pyrene & $0.02 \pm 0.01$ & FB & 0.02 & 0.01 \\
\hline Benz[a]anthracene & $0.003 \pm 0.01$ & FB & 0.002 & 0.002 \\
\hline Chrysene & $0.01 \pm 0.003$ & FB & 0.01 & 0.01 \\
\hline Benzo $[b+k]$ fluoranthene & $0.01 \pm 0.003$ & FB & 0.01 & 0.01 \\
\hline Benzo[e]pyrene & $0.001 \pm 0.001$ & FB & 0.001 & 0.001 \\
\hline Benzola]pyrene & $0.001 \pm 0.001$ & FB & 0.001 & 0.002 \\
\hline Indeno[ $[1,2,3-c d]$ pyrene & $0.001 \pm 0.001$ & $\mathrm{FB}$ & 0.001 & 0.001 \\
\hline Dibenz[ah]anthracene & $0.001 \pm 0.0003$ & FB & 0.001 & 0.001 \\
\hline Benzo[ghi]perylene & $0.001 \pm 0.001$ & FB & 0.001 & 0.001 \\
\hline
\end{tabular}

a Means and SDs are based on three measurements. In cases where one or two values were less than the detection limit, then one-half of the detection limit was used to calculate the mean and SD.

- " $P$ " indicates the results generally passed three indicators of data quality, and " $F$ " indicates significant failure in one of the indicators to the degree that the results should not be trusted; a listing of " $B$ " indicates that some of the blank values exceeded $50 \%$ of the measured values. " $S$ " and " $A$ " indicate that the contractor generally failed to meet method recovery criteria for surrogate (or spiked) analytes or audit samples, respectively. " $Q$ " indicates emission factors are highly questionable since one or two out of three samples were below the detection limit or because insufficient information was available on either blank, spike, or audit tests. 
APPENDIX C

RADIONUCLIDE DATA FOR COAL AND STACK GAS SAMPLES 
Table C-1. Coal and Stack Data for Pb-210

\begin{tabular}{|c|c|c|c|c|c|c|c|c|c|c|c|c|}
\hline \multirow[b]{2}{*}{$\begin{array}{l}\text { Station or } \\
\text { Plant }\end{array}$} & \multicolumn{3}{|c|}{ Coal Concentration (values in $\mathrm{pCi} / \mathrm{g}$ ) } & \multicolumn{4}{|c|}{$\begin{array}{c}\text { Stack Concentrations (values in } \mathrm{pCi} / \mathrm{Nm}^{3} \text { unless } \\
\text { otherwise noted) }\end{array}$} & \multirow{2}{*}{$\begin{array}{l}\text { Emission } \\
\text { Factor, } \\
\mathrm{Ib} / 10^{12} \mathrm{Btu}\end{array}$} & \multirow{2}{*}{$\begin{array}{c}\text { Emission } \\
\text { Factor } \\
95 \% \mathrm{Cl}\end{array}$} & \multirow{2}{*}{$\begin{array}{l}\text { Emission } \\
\text { Factor, } \\
\mathrm{pCl} / 10^{12} \text { Btu }\end{array}$} & \multirow{2}{*}{$\begin{array}{c}\text { Emission } \\
\text { Factor } \\
95 \% \mathrm{Cl}\end{array}$} & \multirow{2}{*}{$\begin{array}{c}\text { Control } \\
\text { Device } \\
\% \\
\text { Remova }\end{array}$} \\
\hline & $\begin{array}{l}\text { Individual } \\
\text { Values }\end{array}$ & Mean & $\begin{array}{l}\text { Standard } \\
\text { Deviation }\end{array}$ & $\begin{array}{l}\text { Individual } \\
\text { Values }\end{array}$ & Mean & $\begin{array}{l}\text { Standard } \\
\text { Deviation }\end{array}$ & Blank Range & & & & & \\
\hline Bailly & $\begin{array}{l}1.3 \\
1.5 \\
0.8\end{array}$ & 1.2 & 0.16 & NA & NA & NA & NA & NA & NA & NA & NA & NA \\
\hline Baldwin & $\begin{array}{c}N D<0.62 \\
0.92 \\
N D<0.66\end{array}$ & 0.52 & NA & $\begin{array}{l}N D<47 \\
N D<31 \\
N D<41\end{array}$ & $N D<40$ & NA & NA & $N D<3.8 \times 10^{-7}$ & $3.8 \times 10^{-7}$ & $N D<1.3 \times 10^{10}$ & $1.3 \times 10^{10}$ & $>64$ \\
\hline Boswell & $\begin{array}{l}N D<0.62 \\
N D<0.60 \\
N D<0.61\end{array}$ & ND $<0.53$ & NA & $\begin{array}{l}N D<4.0 \\
N D<4.1 \\
N D<3.4\end{array}$ & $N D<3.8$ & NA & NA & $N D<3.9 \times 10^{-8}$ & $3.9 \times 10^{-8}$ & ND $<1.4 \times 10^{9}$ & $1.4 \times 10^{9}$ & $>87$ \\
\hline Cardinal* & $\begin{array}{l}2.7 \\
1.6 \\
1.7\end{array}$ & 2.0 & 0.61 & $\begin{array}{l}230 \\
150 \\
250\end{array}$ & 210 & 51 & NA & $2.1 \times 10^{-8}$ & $2.3 \times 10^{-15}$ & $7.2 \times 10^{10}$ & 79 & 54 \\
\hline Coal Creek & $\begin{array}{c}0.57 \\
0.65 \\
1.1\end{array}$ & 0.76 & 0.26 & $\begin{array}{c}270 \\
N D<220 \\
N D<220\end{array}$ & $N D<220$ & NA & $N D<280$ & $N D<1.6 \times 10^{-6}$ & $1.6 \times 10^{-8}$ & $N D<5.5 \times 10^{10}$ & $5.5 \times 10^{10}$ & NA \\
\hline Niles & $\begin{array}{l}2.2 \\
1.6 \\
1.4\end{array}$ & 1.7 & 0.43 & $\begin{array}{l}N D<850 \\
N D<360 \\
N D<540\end{array}$ & $N D<590$ & NA & $N D<440$ & $\mathrm{ND}<4.7 \times 10^{-7}$ & $4.7 \times 10^{-7}$ & $N D<1.6 \times 10^{10}$ & $1.6 \times 10^{10}$ & NA \\
\hline SNOX & $\begin{array}{l}2.0 \\
1.6 \\
2.4\end{array}$ & 2.0 & 0.40 & $\begin{array}{l}N D<200 \\
N D<170 \\
N D<490\end{array}$ & $N D<290$ & NA & $N D<420$ & $N D<94$ & 94 & $N D<3.2 \times 10^{18}$ & $3.2 \times 10^{18}$ & NA \\
\hline Springerville & $\begin{array}{l}0.0 \\
0.6 \\
1.1\end{array}$ & 0.57 & 0.14 & NA & NA & NA & NA & NA & NA & NA & NA & NA \\
\hline Yates & $\begin{array}{l}1.2 \\
1.0 \\
1.7 \\
1.0\end{array}$ & 1.3 & 0.28 & NA & NA & NA & NA & NA & NA & NA & NA & NA \\
\hline
\end{tabular}

\footnotetext{
$\mathrm{NA}=$ not available, $\mathrm{ND}=$ not detected, " stack concentrations at Cardinal in pCi/dscm @ $3 \% \mathrm{O}_{2}$
} 
Table C-2. Coal and Stack Data for Pb-211

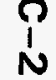

\begin{tabular}{|c|c|c|c|c|c|c|c|c|c|c|c|c|}
\hline \multirow[b]{2}{*}{$\begin{array}{l}\text { Station or } \\
\text { Plant }\end{array}$} & \multicolumn{3}{|c|}{ Coal Concentration (values in $\mathrm{pCi} / \mathrm{g}$ ) } & \multicolumn{4}{|c|}{ Stack Concentrations (values in $\mathrm{pCi} / \mathrm{Nm}^{3}$ ) } & \multirow{2}{*}{$\begin{array}{l}\text { Emission } \\
\text { Factor, } \\
\text { Ib/10 } 10^{12} \text { Btu }\end{array}$} & \multirow{2}{*}{$\begin{array}{l}\text { Emission } \\
\text { Factor } \\
95 \% \mathrm{Cl}\end{array}$} & \multirow{2}{*}{$\begin{array}{c}\text { Emission } \\
\text { Factor, } \\
\text { pCi } / 10^{12} \text { Btu }\end{array}$} & \multirow{2}{*}{$\begin{array}{l}\text { Emission } \\
\text { Factor } \\
95 \% \mathrm{Cl}\end{array}$} & \multirow{2}{*}{$\begin{array}{c}\text { Control } \\
\text { Device } \\
\% \\
\text { Removal }\end{array}$} \\
\hline & $\begin{array}{l}\text { Individual } \\
\text { Values }\end{array}$ & Mean & $\begin{array}{l}\text { Standard } \\
\text { Deviation }\end{array}$ & $\begin{array}{l}\text { Individual } \\
\text { Values }\end{array}$ & Mean & $\begin{array}{l}\text { Standard } \\
\text { Deviation }\end{array}$ & Blank Range & & & & & \\
\hline \multirow[t]{2}{*}{ Bailly } & & & & & & & & & & & & \\
\hline & NA & NA & NA & NA & NA & NA & NA & NA & NA & NA & NA & NA \\
\hline \multirow[t]{2}{*}{ Baldwin } & & & & & & & & & & & & \\
\hline & NA & NA & NA & NA & NA & NA & NA & NA & NA & NA & NA & NA \\
\hline \multirow[t]{2}{*}{ Boswell } & & & & & & & & & & & & \\
\hline & NA & NA & NA & NA & NA & NA & NA & NA & NA & NA & NA & NA \\
\hline \multirow[t]{2}{*}{ Cardinal } & & & & & & & & & & & & \\
\hline & NA & NA & NA & NA & NA & NA & NA & NA & NA & NA & NA & NA \\
\hline Coal Creek & $\begin{array}{l}N D<0.78 \\
N D<0.98 \\
N D<0.80\end{array}$ & ND $<0.85$ & NA & $\begin{array}{l}N D<230 \\
N D<220 \\
N D<240\end{array}$ & ND $<230$ & NA & ND $<310$ & $N D<7.0 \times 10^{.12}$ & $7.0 \times 10^{-12}$ & ND $<7.9 \times 10^{10}$ & $7.9 \times 10^{10}$ & NA \\
\hline Niles & $\begin{array}{l}N D<1.6 \\
N D<1.4 \\
N D<1.4\end{array}$ & $N D<1.5$ & NA & $\begin{array}{c}N D<1400 \\
N D<540 \\
N D<600\end{array}$ & $N D<860$ & NA & ND $<590$ & $N D<2.1 \times 10^{-11}$ & $2.1 \times 10^{-11}$ & $N D>2.4 \times 10^{11}$ & $2.4 \times 10^{11}$ & NA \\
\hline SNOX & $\begin{array}{l}N D<2.2 \\
N D<1.5 \\
N D<1.7\end{array}$ & ND $<1.8$ & NA & $\begin{array}{l}N D<300 \\
N D<270 \\
N D<520\end{array}$ & $N D<360$ & NA & $N D<440$ & ND $<120$ & 120 & $N D<1.4 \times 10^{24}$ & $1.4 \times 10^{24}$ & NA \\
\hline Springerville & & & & & & & & & & & & \\
\hline & NA & NA & NA & NA & NA & NA & NA & NA & NA & NA & NA & NA \\
\hline Yates & & & & & & & & & & & & \\
\hline & NA & NA & NA & NA & NA & NA & NA & NA & NA & NA & NA & NA \\
\hline
\end{tabular}

NA $=$ not available, $N D=$ not detected. 
Table C-3. Coal and Stack Data for Pb-212

\begin{tabular}{|c|c|c|c|c|c|c|c|c|c|c|c|c|}
\hline \multirow[b]{2}{*}{$\begin{array}{l}\text { Station or } \\
\text { Plant }\end{array}$} & \multicolumn{3}{|c|}{ Coal Concentration (values in $\mathrm{pCi} / \mathrm{g}$ ) } & \multicolumn{4}{|c|}{ Stack Concentrations (values in $\mathrm{pCi} / \mathrm{Nm}^{3}$ ) } & \multirow{2}{*}{$\begin{array}{l}\text { Emission } \\
\text { Factor, } \\
\text { lb } / 10^{12} \text { Btu }\end{array}$} & \multirow{2}{*}{$\begin{array}{c}\text { Emission } \\
\text { Factor } \\
95 \% \mathrm{Cl}\end{array}$} & \multirow{2}{*}{$\begin{array}{l}\text { Emission } \\
\text { Factor, } \\
\text { pCi } / 10^{12} \text { Btu }\end{array}$} & \multirow{2}{*}{$\begin{array}{l}\text { Emission } \\
\text { Factor } \\
95 \% \mathrm{Cl}\end{array}$} & \multirow{2}{*}{$\begin{array}{c}\text { Control } \\
\text { Device } \\
\% \\
\text { Removal }\end{array}$} \\
\hline & $\begin{array}{l}\text { Individual } \\
\text { Values }\end{array}$ & Mean & $\begin{array}{l}\text { Standard } \\
\text { Deviation }\end{array}$ & $\begin{array}{l}\text { Individual } \\
\text { Values }\end{array}$ & Mean & $\begin{array}{l}\text { Standard } \\
\text { Deviation }\end{array}$ & $\begin{array}{l}\text { Blank } \\
\text { Range }\end{array}$ & & & & & \\
\hline \multicolumn{13}{|l|}{ Bailly } \\
\hline & NA & NA & NA & NA & NA & NA & NA & NA & NA & NA & NA & NA \\
\hline \multicolumn{13}{|l|}{ Baldwin } \\
\hline & NA & NA & NA & NA & NA & NA & NA & NA & NA & NA & NA & NA \\
\hline \multicolumn{13}{|l|}{ Boswell } \\
\hline & NA & NA & NA & NA & NA & NA & NA & NA & NA & NA & NA & NA \\
\hline \multicolumn{13}{|l|}{ Cardinal } \\
\hline & NA & NA & NA & NA & NA & NA & NA & NA & NA & NA & NA & NA \\
\hline Coal Creek & $\begin{array}{l}0.19 \\
0.24 \\
0.21\end{array}$ & 0.21 & 0.03 & $\begin{array}{l}N D<21 \\
22 \\
N D<19\end{array}$ & ND $<21$ & NA & $N D<26$ & $N D<7.6 \times 10^{-12}$ & $7.6 \times 10^{-12}$ & $N D<4.8 \times 10^{9}$ & $4.8 \times 10^{9}$ & NA \\
\hline Niles & $\begin{array}{l}0.33 \\
0.38 \\
0.33\end{array}$ & 0.35 & 0.03 & $\begin{array}{l}N D<85 \\
N D<38 \\
N D<36\end{array}$ & $N D<53$ & NA & $N D<37$ & $N D<2.4 \times 10^{-11}$ & $2.4 \times 10^{-11}$ & $N D<1.5 \times 10^{10}$ & $1.5 \times 10^{10}$ & NA \\
\hline SNOX & $\begin{array}{l}0.27 \\
0.30 \\
0.29\end{array}$ & 0.28 & 0.02 & $\begin{array}{l}N D<16 \\
N D<20 \\
N D<39\end{array}$ & ND $<25$ & NA & $N D<26$ & $N D<8.3$ & 8.3 & $N D<5.2 \times 10^{21}$ & $5.2 \times 10^{21}$ & NA \\
\hline \multicolumn{13}{|l|}{ Springerville } \\
\hline & NA & NA & NA & NA & NA & NA & NA & NA & NA & NA & NA & NA \\
\hline Yates & $\begin{array}{l}0.20 \\
0.20 \\
0.20 \\
0.20\end{array}$ & 0.20 & 0.00 & NA & NA & NA & NA & NA & NA & NA & NA & NA \\
\hline
\end{tabular}

NA $=$ not available, $N D=$ not detected 


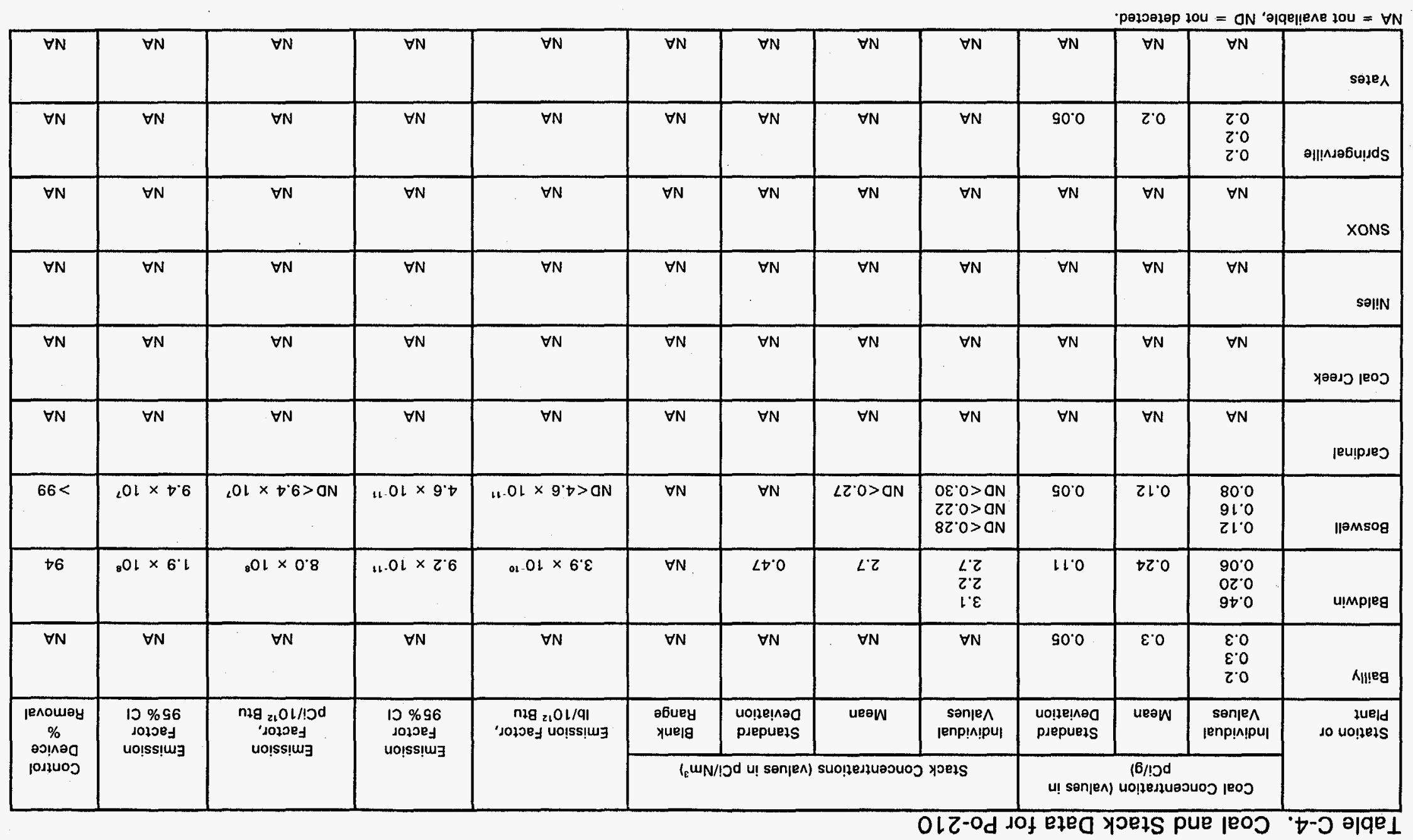


Table C-5. Coal and Stack Data for Ra-226

\begin{tabular}{|c|c|c|c|c|c|c|c|c|c|c|c|c|}
\hline \multirow[b]{2}{*}{$\begin{array}{l}\text { Station or } \\
\text { Plant }\end{array}$} & \multicolumn{3}{|c|}{ Coal Concentration (values in $\mathrm{pCi} / \mathrm{g}$ ) } & \multicolumn{4}{|c|}{$\begin{array}{l}\text { Stack Concentrations (values in } \mathrm{pCi} / \mathrm{Nm}^{3} \text { unless } \\
\text { otherwise noted) }\end{array}$} & \multirow[b]{2}{*}{$\begin{array}{l}\text { Emission Factor, } \\
\mathrm{Ib} / 10^{12} \mathrm{Btu}\end{array}$} & \multirow{2}{*}{$\begin{array}{c}\text { Emission } \\
\text { Factor } \\
95 \% \mathrm{Cl}\end{array}$} & \multirow{2}{*}{$\begin{array}{c}\text { Emission } \\
\text { Factor, } \\
\mathrm{pCi} / 10^{12} \text { Btu }\end{array}$} & \multirow{2}{*}{$\begin{array}{c}\text { Emission } \\
\text { Factor } \\
95 \% \mathrm{Cl}\end{array}$} & \multirow{2}{*}{$\begin{array}{c}\text { Control } \\
\text { Device } \\
\% \\
\text { Remova }\end{array}$} \\
\hline & $\begin{array}{l}\text { Individual } \\
\text { Values }\end{array}$ & Mean & $\begin{array}{l}\text { Standard } \\
\text { Deviation }\end{array}$ & $\begin{array}{l}\text { Individual } \\
\text { Values }\end{array}$ & Mean & $\begin{array}{l}\text { Standard } \\
\text { Deviation }\end{array}$ & $\begin{array}{l}\text { Blank } \\
\text { Range }\end{array}$ & & & & & \\
\hline Bailly & $\begin{array}{l}0.8 \\
0.7 \\
0.7\end{array}$ & 0.7 & 0.12 & NA & NA & NA & NA & NA & NA & NA & NA & NA \\
\hline Baldwin & $\begin{array}{l}0.40 \\
0.25 \\
0.65\end{array}$ & 0.43 & 0.11 & $\begin{array}{l}N D<0.31 \\
N D<0.25 \\
N D<0.20\end{array}$ & $N D<0.26$ & NA & NA & $N D<1.9 \times 10^{-7}$ & $1.9 \times 10^{-7}$ & $N D<8.6 \times 10^{7}$ & $8.6 \times 10^{7}$ & $>99$ \\
\hline Boswell & $\begin{array}{l}0.21 \\
0.27 \\
0.35\end{array}$ & 0.28 & 0.11 & $\begin{array}{l}0.46 \\
0.21 \\
0.54\end{array}$ & 0.40 & 0.17 & NA & $3.2 \times 10^{-7}$ & $3.4 \times 10^{.7}$ & $1.4 \times 10^{8}$ & $1.5 \times 10^{8}$ & 98 \\
\hline Cardinal* & $\begin{array}{l}0.68 \\
0.61 \\
0.69\end{array}$ & 0.66 & 0.04 & $\begin{array}{l}N D<20 \\
N D<19 \\
N D<17\end{array}$ & ND $<18$ & NA & NA & $N D<1.4 \times 10^{-6}$ & $1.4 \times 10^{-6}$ & ND $<6.3 \times 10^{\circ}$ & $6.3 \times 10^{9}$ & 61 \\
\hline Coal Creek & $\begin{array}{c}N D<0.16 \\
0.52 \\
0.50\end{array}$ & 0.37 & NA & $\begin{array}{c}N D<25 \\
13 \\
N D<22\end{array}$ & $N D<25$ & NA & ND $<31$ & $N D<9.1 \times 10^{-8}$ & $1.6 \times 10^{-6}$ & $N D<4.1 \times 10^{9}$ & $7.2 \times 10^{8}$ & NA \\
\hline Niles & $\begin{array}{l}0.48 \\
0.54 \\
0.45\end{array}$ & 0.49 & 0.05 & $\begin{array}{l}N D<120 \\
N D<40 \\
N D<36\end{array}$ & $N D<66$ & NA & $N D<53$ & $N D<4.0 \times 10^{-6}$ & $4.0 \times 10^{-6}$ & $N D<1.8 \times 10^{10}$ & $1.8 \times 10^{10}$ & NA \\
\hline SNOX & $\begin{array}{l}0.48 \\
0.41 \\
0.59\end{array}$ & 0.50 & 0.09 & $\begin{array}{l}N D<28 \\
N D<29 \\
N D<49\end{array}$ & $N D<35$ & NA & $N D<58$ & $N D<12$ & 12 & $N D<5.4 \times 10^{15}$ & $5.4 \times 10^{15}$ & NA \\
\hline Springerville & $\begin{array}{l}0.0 \\
0.2 \\
0.4\end{array}$ & 0.2 & 0.05 & NA & NA & NA & NA & NA & NA & NA & NA & NA \\
\hline Yates & $\begin{array}{l}1.0 \\
1.5 \\
1.0 \\
1.6\end{array}$ & 1.2 & 0.23 & NA & NA & NA & NA & NA & NA & NA & NA & NA \\
\hline
\end{tabular}

$\mathrm{NA}=$ not available, $\mathrm{ND}=$ not detected, " stack concentrations for Cardinal in pCi/dscm @ $3 \% \mathrm{O}_{2}$. 
Table C-6. Coal and Stack Data for Ra-228

i

\begin{tabular}{|c|c|c|c|c|c|c|c|c|c|c|c|c|}
\hline \multirow[b]{2}{*}{$\begin{array}{l}\text { Station or } \\
\text { Plant }\end{array}$} & \multicolumn{3}{|c|}{ Coal Concentration (values in $\mathrm{pC} / \mathrm{g}$ ) } & \multicolumn{4}{|c|}{$\begin{array}{c}\text { Stack Concentrations (values in } \mathrm{pCi} / \mathrm{Nm}^{3} \text { unless } \\
\text { otherwise noted) }\end{array}$} & \multirow{2}{*}{$\begin{array}{l}\text { Emission } \\
\text { Factor, } \\
\text { lb/10 } 10^{\prime 2} \text { Btu }\end{array}$} & \multirow{2}{*}{$\begin{array}{c}\text { Emission } \\
\text { Factor } \\
95 \% \mathrm{Cl}\end{array}$} & \multirow{2}{*}{$\begin{array}{l}\text { Emission } \\
\text { Factor, } \\
\mathrm{pCi} / 10^{12} \text { Btu }\end{array}$} & \multirow{2}{*}{$\begin{array}{l}\text { Emission } \\
\text { Factor } \\
95 \% \mathrm{Cl}\end{array}$} & \multirow{2}{*}{$\begin{array}{c}\text { Control } \\
\text { Device } \\
\% \\
\text { Remova }\end{array}$} \\
\hline & $\begin{array}{l}\text { Individual } \\
\text { Values }\end{array}$ & Mean & $\begin{array}{l}\text { Standard } \\
\text { Deviation }\end{array}$ & $\begin{array}{l}\text { Individual } \\
\text { Values }\end{array}$ & Mean & $\begin{array}{l}\text { Standard } \\
\text { Deviation }\end{array}$ & $\begin{array}{l}\text { Blank } \\
\text { Range }\end{array}$ & & & & & \\
\hline Bailly & $\begin{array}{l}1.1 \\
4.0 \\
0.7\end{array}$ & 1.9 & 0.42 & NA & NA & NA & NA & NA & NA & NA & NA & NA \\
\hline Baldwin & $\begin{array}{l}0.35 \\
0.41 \\
0.43\end{array}$ & 0.40 & 0.28 & $\begin{array}{l}\text { ND }<3.2 \\
\text { ND }<3.3 \\
\text { ND }<6.5\end{array}$ & $N D<4.3$ & NA & NA & $N D<1.2 \times 10^{-8}$ & $1.2 \times 10^{8}$ & $N D<1.5 \times 10^{9}$ & $1.5 \times 10^{9}$ & $>85$ \\
\hline Boswell & $\begin{array}{c}1.1 \\
0.58 \\
0.52\end{array}$ & 0.74 & 0.33 & $\begin{array}{l}\text { ND }<3.0 \\
\text { ND }<3.4 \\
\text { ND }<2.8\end{array}$ & $N D<3.1$ & NA & NA & $N D<8.8 \times 10^{9}$ & $8.8 \times 10^{9}$ & $N D<1.1 \times 10^{9}$ & $1.1 \times 10^{9}$ & $>86$ \\
\hline Cardinal* & $\begin{array}{c}N D<0.53 \\
0.57 \\
0.53\end{array}$ & 0.46 & NA & $\begin{array}{l}N D<46 \\
N D<37 \\
N D<45\end{array}$ & $N D<43$ & NA & NA & $N D<1.2 \times 10^{-7}$ ? & $1.2 \times 10^{-7}$ & $N D<1.5 \times 10^{10}$ & $1.5 \times 10^{10}$ & NA \\
\hline Coal Creek & $\begin{array}{c}0.18 \\
0.27 \\
\text { ND }<0.21\end{array}$ & ND $<0.21$ & NA & $\begin{array}{l}N D<41 \\
N D<46 \\
N D<41\end{array}$ & $N D<43$ & NA & $N D<69$ & $N D<1.1 \times 10^{-7}$ & $1.1 \times 10^{7}$ & $N D<1.4 \times 10^{10}$ & $1.4 \times 10^{10}$ & NA \\
\hline Niles & $\begin{array}{c}N D<0.47 \\
0.27 \\
\text { ND }<0.33\end{array}$ & ND $<0.47$ & NA & $\begin{array}{l}N D<280 \\
N D<120 \\
N D<120\end{array}$ & $N D<170$ & NA & $N D<140$ & $N D<3.9 \times 10^{-7}$ & $3.9 \times 10^{-7}$ & $N D<4.8 \times 10^{10}$ & $4.8 \times 10^{10}$ & NA \\
\hline SNOX & $\begin{array}{l}N D<0.52 \\
N D<0.26 \\
N D<0.28\end{array}$ & $N D<0.35$ & NA & $\begin{array}{c}N D<72 \\
N D<62 \\
N D<110\end{array}$ & ND $<82$ & NA & ND $<97$ & $N D<27$ & 27 & $N D<3.4 \times 10^{18}$ & $3.4 \times 10^{18}$ & NA \\
\hline Springerville & $\begin{array}{l}0.8 \\
0.0 \\
0.6\end{array}$ & 0.47 & 0.34 & NA & NA & NA & NA & NA & NA & NA & NA & NA \\
\hline Yates & NA & NA & NA & NA & NA & NA & NA & NA & NA & NA & NA & NA \\
\hline
\end{tabular}

$\mathrm{NA}=$ not available, $\mathrm{ND}=$ not detected, " stack concentrations for Cardinal reported as $\mathrm{pCi} / \mathrm{dscm} @ 3 \% \mathrm{O}_{2}$. 
Table C-7. Coal and Stack Data for Th-228

\begin{tabular}{|c|c|c|c|c|c|c|c|c|c|c|c|c|}
\hline \multirow[b]{2}{*}{$\begin{array}{l}\text { Station or } \\
\text { Plant }\end{array}$} & \multicolumn{3}{|c|}{ Coal Concentration (values in $\mathrm{pCi} / \mathrm{g}$ ) } & \multicolumn{4}{|c|}{ Stack Concentrations (values in $\mathrm{pCi} / \mathrm{Nm}^{3}$ ) } & \multirow[b]{2}{*}{$\begin{array}{l}\text { Emission Factor, } \\
\mathrm{lb} / 10^{12} \mathrm{Btu}\end{array}$} & \multirow{2}{*}{$\begin{array}{c}\text { Emission } \\
\text { Factor } \\
95 \% \mathrm{Cl}\end{array}$} & \multirow{2}{*}{$\begin{array}{c}\text { Emission } \\
\text { Factor, } \\
\mathrm{pCi} / 10^{12} \mathrm{Btu}\end{array}$} & \multirow{2}{*}{$\begin{array}{l}\text { Emission } \\
\text { Factor, } \\
95 \% \mathrm{Cl}\end{array}$} & \multirow{2}{*}{$\begin{array}{c}\text { Control } \\
\text { Device } \\
\% \\
\text { Remova }\end{array}$} \\
\hline & $\begin{array}{l}\text { Individual } \\
\text { Values }\end{array}$ & Mean & $\begin{array}{l}\text { Standard } \\
\text { Deviation }\end{array}$ & $\begin{array}{l}\text { Individual } \\
\text { Values }\end{array}$ & Mean & $\begin{array}{l}\text { Standard } \\
\text { Deviation }\end{array}$ & $\begin{array}{l}\text { Blank } \\
\text { Range }\end{array}$ & & & & & \\
\hline Bailly & $\begin{array}{l}0.4 \\
0.5 \\
0.5\end{array}$ & 0.5 & 0.05 & NA & NA & NA & NA & NA & NA & NA & NA & NA \\
\hline Baldwin & $\begin{array}{l}0.27 \\
0.09 \\
0.17\end{array}$ & 0.18 & 0.05 & $\begin{array}{c}1.41 \\
N D<3.4 \\
N D<1.6\end{array}$ & $N D<3.4$ & NA & NA & $N D<3.2 \times 10^{-9}$ & $3.2 \times 10^{-9}$ & $N D<1.2 \times 10^{9}$ & $N D<1.2 \times 10^{9}$ & $>53$ \\
\hline Boswell & $\begin{array}{l}0.18 \\
0.18 \\
0.15\end{array}$ & 0.17 & 0.05 & $\begin{array}{c}N D<0.058 \\
N D<0.18 \\
\text { ND }<0.085\end{array}$ & $N D<0.11$ & NA & NA & $N D<1.0 \times 10^{-10}$ & $1.0 \times 10^{-10}$ & $N D<3.7 \times 10^{7}$ & $3.7 \times 10^{7}$ & $>99$ \\
\hline Cardinal & NA & NA & NA & NA & NA & NA & NA & NA & NA & NA & NA & NA \\
\hline Coal Creek & NA & NA & NA & NA & NA & NA & NA & NA & NA & NA & NA & NA \\
\hline Niles & NA & NA & NA & NA & NA & NA & NA & NA & NA & NA & NA & NA \\
\hline SNOX & NA & NA & NA & NA & NA & NA & NA & NA & NA & NA & NA & NA \\
\hline Springerville. & $\begin{array}{l}0.4 \\
0.5 \\
0.6\end{array}$ & 0.5 & 0.09 & NA & NA & NA & NA & NA & NA & NA & NA & NA \\
\hline Yates & NA & NA & NA & NA & NA & NA & NA & NA & NA & NA & NA & NA \\
\hline
\end{tabular}

$\mathrm{NA}=$ not available, $\mathrm{ND}=$ not detected 
Table C-8. Coal and Stack Data for Th-229

\begin{tabular}{|c|c|c|c|c|c|c|c|c|c|c|c|c|}
\hline \multirow[b]{2}{*}{$\begin{array}{l}\text { Station or } \\
\text { Plant }\end{array}$} & \multicolumn{3}{|c|}{ Coal Concentration (values in $\mathrm{pCi} / \mathrm{g}$ ) } & \multicolumn{4}{|c|}{$\begin{array}{l}\text { Stack Concentrations (values in pCi/ } \mathrm{Nm}^{3} \text { unless } \\
\text { otherwise noted) }\end{array}$} & \multirow{2}{*}{$\begin{array}{l}\text { Emission } \\
\text { Factor, } \\
\mathrm{Ib} / 10^{12} \mathrm{Btu}\end{array}$} & \multirow{2}{*}{$\begin{array}{l}\text { Emission } \\
\text { Factor } \\
95 \% \mathrm{Cl}\end{array}$} & \multirow{2}{*}{$\begin{array}{l}\text { Emission } \\
\text { Factor, } \\
\text { pCi/10 }\end{array}$} & \multirow{2}{*}{$\begin{array}{l}\text { Emission } \\
\text { Factor, } \\
95 \% \mathrm{Cl}\end{array}$} & \multirow{2}{*}{$\begin{array}{c}\text { Control } \\
\text { Device } \\
\% \\
\text { Removal }\end{array}$} \\
\hline & $\begin{array}{l}\text { Individual } \\
\text { Values }\end{array}$ & Mean & $\begin{array}{l}\text { Standard } \\
\text { Deviation }\end{array}$ & $\begin{array}{l}\text { Individual } \\
\text { Values }\end{array}$ & Mean & $\begin{array}{l}\text { Standard } \\
\text { Deviation }\end{array}$ & Blank Range & & & & & \\
\hline Bailly & NA & NA & NA & NA & NA & NA & NA & NA & NA & NA & NA & NA \\
\hline Baldwin & NA & NA & NA & NA & NA & NA & NA & NA & NA & NA & NA & NA \\
\hline Boswell & NA & NA & NA & NA & NA & NA & NA & NA & NA & NA & NA & NA \\
\hline Cardinal & NA & NA & NA & NA & NA & NA & NA & NA & NA & NA & NA & NA \\
\hline Coal Creek & $\begin{array}{l}N D<0.29 \\
N D<0.31 \\
N D<0.28\end{array}$ & $N D<0.29$ & NA & $\begin{array}{l}N D<90 \\
N D<99 \\
N D<87\end{array}$ & ND $<92$ & NA & $N D<140$ & $\mathrm{ND}<3.2 \times 10^{-4}$ & $3.2 \times 10^{-4}$ & $N D<3.1 \times 10^{10}$ & $3.1 \times 10^{10}$ & NA \\
\hline Niles & $\begin{array}{l}N D<0.58 \\
N D<0.58 \\
N D<0.57\end{array}$ & $N D<0.58$ & NA & $\begin{array}{l}N D<520 \\
N D<200 \\
N D<280\end{array}$ & $N D<330$ & NA & $N D<260$ & $N D<9.5 \times 10^{-4}$ & $9.5 \times 10^{-4}$ & $N D<9.2 \times 10^{10}$ & $9.2 \times 10^{10}$ & NA \\
\hline SNOX & $\begin{array}{l}N D<0.8 \\
N D<0.66 \\
N D<0.69\end{array}$ & $N D<0.72$ & NA & $\begin{array}{l}N D<130 \\
N D<130 \\
N D<200\end{array}$ & $N D<150$ & NA & $N D<220$ & $N D<50$ & 50 & $N D<4.9 \times 10^{15}$ & $4.9 \times 10^{15}$ & NA \\
\hline Springerville & NA & NA & NA & NA & NA & NA & NA & NA & NA & NA & NA & NA \\
\hline Yates & NA & NA & NA & NA & NA & NA & NA & NA & NA & NA & NA & NA \\
\hline
\end{tabular}

NA $=$ not available, $N D=$ not detected. 
Table C-9. Coal and Stack Data for Th-230

\begin{tabular}{|c|c|c|c|c|c|c|c|c|c|c|c|c|}
\hline \multirow[b]{2}{*}{$\begin{array}{l}\text { Station or } \\
\text { Plant }\end{array}$} & \multicolumn{3}{|c|}{ Coal Concentration (values in pCi/g) } & \multicolumn{4}{|c|}{$\begin{array}{c}\text { Stack Concentrations (values in } \mathrm{pCi} / \mathrm{Nm}^{3} \text { unless } \\
\text { otherwise noted) }\end{array}$} & \multirow[b]{2}{*}{$\begin{array}{l}\text { Emission Factor, } \\
\mathrm{lb} / 10^{12} \mathrm{Btu}\end{array}$} & \multirow{2}{*}{$\begin{array}{l}\text { Emission } \\
\text { Factor } \\
95 \% \mathrm{Cl}\end{array}$} & \multirow{2}{*}{$\begin{array}{l}\text { Emission } \\
\text { Factor, } \\
\mathrm{pCi} / 10^{12} \text { Btu }\end{array}$} & \multirow{2}{*}{$\begin{array}{l}\text { Emission } \\
\text { Factor } \\
95 \% \mathrm{Cl}\end{array}$} & \multirow{2}{*}{$\begin{array}{c}\text { Control } \\
\text { Device } \\
\% \\
\text { Removal }\end{array}$} \\
\hline & $\begin{array}{l}\text { Individual } \\
\text { Values }\end{array}$ & Mean & $\begin{array}{l}\text { Standard } \\
\text { Deviation }\end{array}$ & $\begin{array}{l}\text { Individual } \\
\text { Values }\end{array}$ & Mean & $\begin{array}{l}\text { Standard } \\
\text { Deviation }\end{array}$ & Blank Range & & & & & \\
\hline Bailly & $\begin{array}{l}0.8 \\
0.8 \\
0.7\end{array}$ & 0.8 & 0.09 & NA & NA & NA & NA & NA & NA & NA & NA & NA \\
\hline Baldwin & $\begin{array}{l}0.97 \\
0.72 \\
0.76\end{array}$ & 0.82 & 0.11 & $\begin{array}{l}4.8 \\
1.4 \\
5.0\end{array}$ & 7.8 & 5.4 & NA & $3.3 \times 10^{-4}$ & $6.8 \times 10^{-4}$ & $3.1 \times 10^{9}$ & $6.4 \times 10^{9}$ & 73 \\
\hline Boswell & $\begin{array}{l}0.25 \\
0.28 \\
0.22\end{array}$ & 0.25 & 0.05 & $\begin{array}{l}N D<0.058 \\
N D<0.079 \\
N D<0.085\end{array}$ & ND $<0.074$ & NA & NA & $\mathrm{ND}<2.8 \times 10^{-6}$ & $1.0 \times 10^{-6}$ & $N D<2.6 \times 10^{7}$ & $2.6 \times 10^{7}$ & $>99$ \\
\hline Cardinal " & $\begin{array}{l}N D<7.3 \\
N D<7.2 \\
N D<7.3\end{array}$ & $\mathrm{ND}<7.3$ & NA & $\begin{array}{l}N D<830 \\
N D<790 \\
N D<710\end{array}$ & ND $<780$ & NA & NA & $N D<2.8 \times 10^{-2}$ & $2.8 \times 10^{-2}$ & $N D<2.6 \times 10^{11}$ & $2.6 \times 10^{11}$ & NA \\
\hline Coal Creek & $\begin{array}{l}\text { ND }<3.6 \\
\text { ND }<4.7 \\
\text { ND }<3.7\end{array}$ & ND $<4.0$ & NA & $\begin{array}{c}N D<1000 \\
\text { ND }<1000 \\
\text { ND }<990\end{array}$ & $N D<1000$ & NA & $N D<1400$ & $N D<3.6 \times 10^{-2}$ & $3.6 \times 10^{-2}$ & $N D<3.4 \times 10^{11}$ & $3.4 \times 10^{11}$ & NA \\
\hline Niles & $\begin{array}{l}N D<5.2 \\
\text { ND }<6.9 \\
\text { ND }<6.5\end{array}$ & $N D<6.2$ & NA & $\begin{array}{l}N D<4700 \\
N D<2400 \\
N D<2400\end{array}$ & $N D<3200$ & NA & $N D<2900$ & $N D<9.4 \times 10^{-2}$ & $9.4 \times 10^{-2}$ & ND $<8.810^{\prime \prime}$ & $8.8 \times 10^{11}$ & NA \\
\hline SNOX & $\begin{array}{l}N D<8.7 \\
N D<6.7 \\
\text { ND }<5.9\end{array}$ & $N D<7.1$ & NA & $\begin{array}{l}N D<1200 \\
N D<1200 \\
N D<2500\end{array}$ & $N D<1600$ & NA & $N D<2200$ & $N D<540$ & 540 & $N D<5.1 \times 10^{15}$ & $5.1 \times 10^{15}$ & NA \\
\hline Springerville & $\begin{array}{l}1.2 \\
0.9 \\
1.2\end{array}$ & 1.1 & 0.15 & NA & NA & NA & NA & NA & NA & NA & NA & NA \\
\hline Yates & NA & NA & NA & NA & $\mathrm{NA}{ }^{\circ}$ & NA & NA & NA & NA & NA & NA & NA \\
\hline
\end{tabular}

$\mathrm{NA}=$ not available, ND $=$ not detected, ${ }^{\bullet}$ stack concentration at Cardinal in $\mathrm{pCi} / \mathrm{dscm} @ 3 \% \mathrm{O}_{2}$. 
Table C-10. Coal and Stack Data for Th-232

\begin{tabular}{|c|c|c|c|c|c|c|c|c|c|c|c|c|}
\hline \multirow[b]{2}{*}{$\begin{array}{l}\text { Station or } \\
\text { Plant }\end{array}$} & \multicolumn{3}{|c|}{ Coal Concentration (values in $\mathrm{pCi} / \mathrm{g}$ ) } & \multicolumn{4}{|c|}{ Stack Concentrations (values in $\mathrm{pCi} / \mathrm{Nm}^{3}$ ) } & \multirow{2}{*}{$\begin{array}{l}\text { Emission } \\
\text { Factor, } \\
\mathrm{Ib} / 10^{12} \text { Btu }\end{array}$} & \multirow{2}{*}{$\begin{array}{c}\text { Emission } \\
\text { Factor } \\
95 \% \mathrm{Cl}\end{array}$} & \multirow{2}{*}{$\begin{array}{l}\text { Emission } \\
\text { Factor, } \\
\mathrm{pCl} / 10^{12} \text { Btu }\end{array}$} & \multirow{2}{*}{$\begin{array}{l}\text { Emission } \\
\text { Factor } \\
95 \% \mathrm{Cl}\end{array}$} & \multirow{2}{*}{$\begin{array}{c}\text { Control } \\
\text { Device } \\
\% \\
\text { Removal }\end{array}$} \\
\hline & $\begin{array}{l}\text { Individual } \\
\text { Values }\end{array}$ & Mean & $\begin{array}{l}\text { Standard } \\
\text { Deviation }\end{array}$ & $\begin{array}{l}\text { Individual } \\
\text { Values }\end{array}$ & Mean & $\begin{array}{l}\text { Standard } \\
\text { Deviation }\end{array}$ & Blank Range & & & & & \\
\hline Bailly & $\begin{array}{l}0.2 \\
0.2 \\
0.5\end{array}$ & 0.3 & 0.05 & NA & NA & NA & NA & NA & NA & NA & NA & NA \\
\hline Baldwin & $\begin{array}{l}0.27 \\
0.09 \\
0.17\end{array}$ & 0.18 & 0.05 & $\begin{array}{c}1.4 \\
N D<3.4 \\
N D<1.6\end{array}$ & ND $<3.4$ & NA & NA & $N D<24$ & 24 & $N D<1.2 \times 10^{9}$ & $1.2 \times 10^{9}$ & $>53$ \\
\hline Boswell & $\begin{array}{l}0.18 \\
0.18 \\
0.15\end{array}$ & 0.17 & 0.05 & $\begin{array}{c}N D<0.058 \\
N D<0.18 \\
N D<0.085\end{array}$ & ND $<0.11$ & NA & NA & $\mathrm{ND}<0.75$ & 0.75 & $N D<3.8 \times 10^{7}$ & $3.8 \times 10^{7}$ & $>99$ \\
\hline Cardinal & NA & NA & NA & NA & NA & NA & NA & NA & NA & NA & NA & NA \\
\hline Coal Creek & NA & NA & NA & NA & NA & NA & NA & NA & NA & NA & NA & NA \\
\hline Niles & NA & NA & NA & NA & NA & NA & NA & NA & NA & NA & NA & NA \\
\hline SNOX & NA & NA & NA & NA & NA & NA & NA & NA & NA & NA & NA & NA \\
\hline Springerville & $\begin{array}{l}0.6 \\
0.4 \\
0.1\end{array}$ & 0.37 & 0.08 & NA & NA & NA & NA & NA & NA & NA & NA & NA \\
\hline Yates & NA & NA & NA & NA & NA & NA & NA & NA & NA & NA & NA & NA \\
\hline
\end{tabular}

$\mathrm{NA}=$ not available, $N D=$ not detected. 
Table C-11. Coal and Stack Data for Th-234

\begin{tabular}{|c|c|c|c|c|c|c|c|c|c|c|c|c|}
\hline \multirow[b]{2}{*}{$\begin{array}{l}\text { Station or } \\
\text { Plant }\end{array}$} & \multicolumn{3}{|c|}{ Coal Concentration (values in $\mathrm{pCi} / \mathrm{g}$ ) } & \multicolumn{4}{|c|}{ Stack Concentrations (values in $\mathrm{pCi} / \mathrm{Nm}^{3}$ ) } & \multirow{2}{*}{$\begin{array}{l}\text { Emission } \\
\text { Factor, } \\
\text { Ib } / 10^{12} \text { Btu }\end{array}$} & \multirow{2}{*}{$\begin{array}{c}\text { Emission } \\
\text { Factor } \\
95 \% \mathrm{Cl}\end{array}$} & \multirow{2}{*}{$\begin{array}{l}\text { Emission } \\
\text { Factor, } \\
\mathrm{pCi} / 10^{12} \mathrm{Btu}\end{array}$} & \multirow{2}{*}{$\begin{array}{c}\text { Emission } \\
\text { Factor } \\
95 \% \mathrm{Cl}\end{array}$} & \multirow{2}{*}{$\begin{array}{c}\text { Control } \\
\text { Device } \\
\% \\
\text { Removal }\end{array}$} \\
\hline & $\begin{array}{l}\text { Individual } \\
\text { Values }\end{array}$ & Mean & $\begin{array}{l}\text { Standard } \\
\text { Deviation }\end{array}$ & $\begin{array}{l}\text { Individual } \\
\text { Values }\end{array}$ & Mean & $\begin{array}{l}\text { Standard } \\
\text { Deviation }\end{array}$ & Blank Range & & & & & \\
\hline \multirow[t]{2}{*}{ Bailly } & & & & & & & & & & & & \\
\hline & NA & NA & NA & NA & NA & NA & NA & NA & NA & NA & NA & NA \\
\hline \multirow[t]{2}{*}{ Baldwin } & & & & & & & & & & & & \\
\hline & NA & NA & NA & NA & NA & NA & NA & NA & NA & NA & NA & NA \\
\hline \multirow[t]{2}{*}{ Boswell } & & & & & & & & & & & & \\
\hline & NA & NA & NA & NA & NA & NA & NA & NA & NA & NA & NA & NA \\
\hline \multirow[t]{2}{*}{ Cardinal } & & & & & & & & & & & & \\
\hline & NA & NA & NA & NA & NA & NA & NA & NA & NA & NA & NA & NA \\
\hline Coal Creek & $\begin{array}{c}0.90 \\
1.2 \\
1.2\end{array}$ & 1.1 & 0.17 & $\begin{array}{l}N D<180 \\
N D<120 \\
N D<140\end{array}$ & $N D<140$ & NA & $N D<220$ & $N D<4.7 \times 10^{-9}$ & $4.7 \times 10^{-9}$ & $N D<4.9 \times 10^{10}$ & $4.9 \times 10^{10}$ & NA \\
\hline Niles & $\begin{array}{l}2.3 \\
3.0 \\
3.0\end{array}$ & 2.8 & 0.38 & $\begin{array}{l}N D<710 \\
N D<300 \\
N D<320\end{array}$ & ND $<440$ & NA & $N D<370$ & $N D<1.2 \times 10^{8}$ & $1.2 \times 10^{-8}$ & ND $<1.3 \times 10^{11}$ & $1.3 \times 10^{11}$ & NA \\
\hline SNOX & $\begin{array}{c}\mathrm{ND}<2.7 \\
3.6 \\
2.5\end{array}$ & ND $<2.7$ & NA & $\begin{array}{c}190 \\
N D<170 \\
N D<310\end{array}$ & $N D<310$ & NA & $N D<400$ & $N D<47$ & 47 & $N D<4.9 \times 10^{20}$ & $4.9 \times 10^{20}$ & NA \\
\hline \multirow[t]{2}{*}{ Springerville } & & & & & & & & & & & & \\
\hline & NA & NA & NA & NA & NA & NA & NA & NA & NA & NA & NA & NA \\
\hline Yates & $\begin{array}{l}0.70 \\
0.50 \\
0.80 \\
0.50\end{array}$ & 0.67 & 0.12 & NA & NA & NA & NA & NA & NA & NA & NA & NA \\
\hline
\end{tabular}

NA $=$ not available, $N D=$ not detected. 
Table C-12. Coal and Stack Data for U-234

\begin{tabular}{|c|c|c|c|c|c|c|c|c|c|c|c|c|}
\hline \multirow[b]{2}{*}{$\begin{array}{l}\text { Station or } \\
\text { Plant }\end{array}$} & \multicolumn{3}{|c|}{ Coal Concentration (values in $\mathrm{pCi} / \mathrm{g}$ ) } & \multicolumn{4}{|c|}{$\begin{array}{c}\text { Stack Concentrations (values in } \mathrm{pCi} / \mathrm{Nm}^{3} \text { unless } \\
\text { otherwise noted) }\end{array}$} & \multirow{2}{*}{$\begin{array}{l}\text { Emission } \\
\text { Factor, } \\
\mathrm{lb} / 10^{12} \text { Btu }\end{array}$} & \multirow{2}{*}{$\begin{array}{c}\text { Emission } \\
\text { Factor } \\
95 \% \mathrm{Cl}\end{array}$} & \multirow{2}{*}{$\begin{array}{c}\text { Emission } \\
\text { Factor, } \\
\text { pCi/10 } 10^{12} \text { Btu }\end{array}$} & \multirow{2}{*}{$\begin{array}{l}\text { Emission } \\
\text { Factor } \\
95 \% \mathrm{Cl}\end{array}$} & \multirow{2}{*}{$\begin{array}{c}\text { Control } \\
\text { Device } \\
\% \\
\text { Removal }\end{array}$} \\
\hline & $\begin{array}{l}\text { Individual } \\
\text { Values }\end{array}$ & Mean & $\begin{array}{l}\text { Standard } \\
\text { Deviation }\end{array}$ & $\begin{array}{l}\text { Individual } \\
\text { Values }\end{array}$ & Mean & $\begin{array}{l}\text { Standard } \\
\text { Deviation }\end{array}$ & Blank Range & & & & & \\
\hline Bailly & $\begin{array}{l}1.5 \\
0.2 \\
1.3\end{array}$ & 1.0 & 0.16 & NA & NA & NA & NA & NA & NA & NA & NA & NA \\
\hline Baldwin & $\begin{array}{l}1.0 \\
1.2 \\
0.96\end{array}$ & 1.1 & 0.11 & $\begin{array}{l}1.6 \\
1.4 \\
1.2\end{array}$ & 1.4 & 0.18 & NA & $1.6 \times 10^{-4}$ & $5.7 \times 10^{5}$ & $4.5 \times 10^{8}$ & $1.6 \times 10^{8}$ & 97 \\
\hline Boswell & $\begin{array}{l}0.37 \\
0.34 \\
0.33\end{array}$ & 0.35 & 0.06 & $\begin{array}{c}N D<0.14 \\
0.12 \\
\text { ND }<0.14\end{array}$ & $N D<0.14$ & NA & NA & $N D<1.9 \times 10^{-5}$ & $1.9 \times 10^{-6}$ & $N D<5.4 \times 10^{7}$ & $5.4 \times 10^{7}$ & $>99$ \\
\hline Cardinal* & $\begin{array}{l}N D<30 \\
N D<25 \\
N D<26\end{array}$ & ND $<27$ & NA & $\begin{array}{l}N D<3700 \\
N D<3700 \\
N D<2900\end{array}$ & $N D<3400$ & NA & NA & $N D<0.42$ & 0.42 & ND $<1.2 \times 10^{12}$ & $1.2 \times 10^{12}$ & NA \\
\hline Coal Creek & $\begin{array}{c}N D<13 \\
12 \\
N D<20\end{array}$ & ND $<20$ & NA & $\begin{array}{l}N D<4400 \\
N D<4400 \\
N D<4600\end{array}$ & $N D<4500$ & NA & ND $<5900$ & $N D<0.54$ & 0.54 & $N D<1.5 \times 10^{12}$ & $1.5 \times 10^{12}$ & NA \\
\hline Niles & $\begin{array}{l}N D<19 \\
N D<23 \\
N D<23\end{array}$ & $N D<22$ & NA & $\begin{array}{l}\text { ND }<22000 \\
\text { ND }<7800 \\
\text { ND }<11000\end{array}$ & $N D<13000$ & NA & ND $<9900$ & ND $<1.3$ & 1.3 & $N D<3.7 \times 10^{12}$ & $3.7 \times 10^{12}$ & NA \\
\hline SNOX & $\begin{array}{c}28 \\
N D<29 \\
N D<24\end{array}$ & $N D<29$ & NA & $\begin{array}{l}N D<4300 \\
N D<4900 \\
N D<10000\end{array}$ & ND $<6500$ & NA & $N D<9700$ & ND $<2100$ & 2100 & $N D<6.0 \times 10^{12}$ & $6.0 \times 10^{12}$ & NA \\
\hline Springerville & $\begin{array}{l}0.0 \\
0.1 \\
0.1\end{array}$ & 0.07 & 0.09 & NA & NA & NA & NA & NA & NA & NA & NA & NA \\
\hline Yates & NA & NA & NA & NA & NA & NA & NA & NA & NA & NA & NA & NA \\
\hline
\end{tabular}

$\mathrm{NA}=$ not available, $\mathrm{ND}=$ not detected, ${ }^{\circ}$ stack concentration at Cardinal in $\mathrm{pCi} / \mathrm{dscm} @ 3 \% \mathrm{O}_{2}$. 
Table C-13. Coal and Stack Data for U-235

\begin{tabular}{|c|c|c|c|c|c|c|c|c|c|c|c|c|}
\hline \multirow[b]{2}{*}{$\begin{array}{l}\text { Station or } \\
\text { Plant }\end{array}$} & \multicolumn{3}{|c|}{ Coal Concentration (values in $\mathrm{pCi} / \mathrm{g}$ ) } & \multicolumn{4}{|c|}{$\begin{array}{c}\text { Stack Concentrations (values in } \mathrm{pCi} / \mathrm{Nm}^{3} \text { unless } \\
\text { otherwise noted) }\end{array}$} & \multirow{2}{*}{$\begin{array}{l}\text { Emission } \\
\text { Factor, } \\
\mathrm{lb} / 10^{12} \mathrm{Btu}\end{array}$} & \multirow{2}{*}{$\begin{array}{c}\text { Emission } \\
\text { Factor } \\
95 \% \mathrm{Cl}\end{array}$} & \multirow{2}{*}{$\begin{array}{l}\text { Emission } \\
\text { Factor, } \\
\text { pCi } / 10^{12} \text { Btu }\end{array}$} & \multirow{2}{*}{$\begin{array}{c}\text { Emission } \\
\text { Factor } \\
95 \% \mathrm{Cl}\end{array}$} & \multirow{2}{*}{$\begin{array}{c}\text { Control } \\
\text { Device } \\
\% \\
\text { Removal }\end{array}$} \\
\hline & $\begin{array}{l}\text { Individual } \\
\text { Values }\end{array}$ & Mean & $\begin{array}{l}\text { Standard } \\
\text { Deviation }\end{array}$ & $\begin{array}{l}\text { Individual } \\
\text { Values }\end{array}$ & Mean & $\begin{array}{l}\text { Standard } \\
\text { Deviation }\end{array}$ & Blank Range & & & & & \\
\hline Bailly & $\begin{array}{c}0.1 \\
N D<0.6 \\
N D<0.4\end{array}$ & 0.2 & NA & NA & NA & NA & NA & NA & NA & NA & NA & NA \\
\hline Baldwin & $\begin{array}{l}0.06 \\
0.05 \\
0.03\end{array}$ & 0.05 & 0.02 & $\begin{array}{l}N D<0.085 \\
N D<0.086 \\
N D<0.15\end{array}$ & $N D<0.11$ & NA & NA & ND $<0.035$ & 0.035 & $N D<3.4 \times 10^{7}$ & $3.4 \times 10^{7}$ & $>95$ \\
\hline Boswell & $\begin{array}{l}0.02 \\
0.02 \\
0.02\end{array}$ & 0.02 & 0.01 & $\begin{array}{l}N D<0.054 \\
N D<0.063 \\
N D<0.14\end{array}$ & ND $<0.087$ & NA & NA & $N D<0.031$ & 0.031 & $N D<3.1 \times 10^{7}$ & $3.1 \times 10^{7}$ & $>95$ \\
\hline Cardinal* & $\begin{array}{l}N D<0.30 \\
N D<0.27 \\
\text { ND }<0.23\end{array}$ & 0.17 & NA & $\begin{array}{c}N D<35 \\
35 \\
N D<35\end{array}$ & 24 & NA & NA & 8.2 & 10 & $8.1 \times 10^{9}$ & $9.8 \times 10^{9}$ & 51 \\
\hline Coal Creek & $\begin{array}{c}N D<0.13 \\
0.12 \\
N D<0.19\end{array}$ & $N D<0.19$ & NA & $\begin{array}{l}N D<41 \\
N D<37 \\
N D<38\end{array}$ & ND $<39$ & NA & $N D<52$ & ND $<13$ & 13 & $N D<1.3 \times 10^{10}$ & $1.3 \times 10^{10}$ & NA \\
\hline Niles & $\begin{array}{l}N D<0.22 \\
N D<0.22 \\
N D<0.23\end{array}$ & $N D<0.22$ & NA & $\begin{array}{l}N D<230 \\
N D<80 \\
N D<110\end{array}$ & $N D<140$ & NA & $N D<110$ & $N D<40$ & 40 & $N D<3.9 \times 10^{10}$ & $3.9 \times 10^{10}$ & NA \\
\hline SNOX & $\begin{array}{l}N D<64 \\
N D<0.26 \\
N D<0.25\end{array}$ & ND $<22$ & NA & $\begin{array}{l}N D<48 \\
N D<540 \\
N D<100\end{array}$ & $N D<230$ & NA & $N D<56$ & ND $<75$ & 75 & $N D<7.4 \times 10^{10}$ & $7.4 \times 10^{10}$ & NA \\
\hline Springerville & $\begin{array}{l}0.0 \\
0.0 \\
0.0\end{array}$ & 0.0 & 0.10 & NA & NA & NA & NA & NA & NA & NA & NA & NA \\
\hline Yates & $\begin{array}{l}0.20 \\
\text { ND } \\
\text { ND } \\
\text { ND }\end{array}$ & 0.07 & NA & NA & NA & NA & NA & NA & NA & NA & NA & NA \\
\hline
\end{tabular}

$\mathrm{NA}=$ not available, $\mathrm{ND}=$ not detected, ${ }^{\bullet}$ stack concentration at Cardinal in $\mathrm{pCi} / \mathrm{dscm} @ 3 \% \mathrm{O}_{2}$. 
Table C-14. Coal and Stack Data for U-238

\begin{tabular}{|c|c|c|c|c|c|c|c|c|c|c|c|c|}
\hline \multirow[b]{2}{*}{$\begin{array}{l}\text { Station or } \\
\text { Plant }\end{array}$} & \multicolumn{3}{|c|}{ Coal Concentration (values in $\mathrm{pCi} / \mathrm{g}$ ) } & \multicolumn{4}{|c|}{$\begin{array}{c}\text { Stack Concentrations (values in } \mathrm{pCi} / \mathrm{Nm}^{3} \text { unless } \\
\text { otherwise noted) }\end{array}$} & \multirow[b]{2}{*}{$\begin{array}{l}\text { Emission } \\
\text { Factor, } \\
\text { lb } / 10^{12} \text { Btu }\end{array}$} & \multirow[b]{2}{*}{$\begin{array}{c}\text { Emission } \\
\text { Factor } \\
95 \% \mathrm{Cl}\end{array}$} & \multirow[b]{2}{*}{$\begin{array}{c}\text { Emission } \\
\text { Factor, } \\
\mathrm{pCl} / 10^{12} \mathrm{Btu}\end{array}$} & \multirow[b]{2}{*}{$\begin{array}{l}\text { Emission } \\
\text { Factor } \\
95 \% \mathrm{Cl}\end{array}$} & \multirow{2}{*}{$\begin{array}{c}\text { Control } \\
\text { Device } \\
\% \\
\text { Removal }\end{array}$} \\
\hline & $\begin{array}{l}\text { Individual } \\
\text { Values }\end{array}$ & Mean & $\begin{array}{l}\text { Standard } \\
\text { Deviation }\end{array}$ & $\begin{array}{l}\text { Individual } \\
\text { Values }\end{array}$ & Mean & $\begin{array}{l}\text { Standard } \\
\text { Deviation }\end{array}$ & Blank Range & & & & & \\
\hline Bailly & $\begin{array}{l}0.5 \\
0.3 \\
0.5\end{array}$ & 0.4 & 0.12 & NA & NA & NA & NA & NA & NA & NA & NA & NA \\
\hline Baldwin & $\begin{array}{l}1.1 \\
1.1 \\
0.91\end{array}$ & 1.0 & 0.10 & $\begin{array}{l}2.1 \\
2.0 \\
1.4\end{array}$ & 1.8 & 0.37 & NA & 4.0 & 2.2 & $6.0 \times 10^{8}$ & $3.3 \times 10^{8}$ & 96 \\
\hline Boswell & $\begin{array}{l}0.37 \\
0.33 \\
0.38\end{array}$ & 0.36 & 0.06 & $\begin{array}{c}\text { ND }<0.054 \\
\text { ND }<0.14 \\
\text { ND }<0.17\end{array}$ & $N D<0.12$ & NA & NA & $N D<0.27$ & 0.31 & $N D<4.1 \times 10^{7}$ & $4.1 \times 10^{7}$ & $>99$ \\
\hline Cardinal* & $\begin{array}{l}4.7 \\
3.9 \\
3.3\end{array}$ & 4.0 & 0.72 & $\begin{array}{l}280 \\
260 \\
280\end{array}$ & 270 & 14 & NA & 620 & 330 & $9.3 \times 10^{10}$ & $5.0 \times 10^{10}$ & 52 \\
\hline Coal Creek & NA & NA & NA & NA & NA & NA & NA & NA & NA & NA & NA & NA \\
\hline Niles & NA & NA & NA & NA & NA & NA & NA & NA & NA & NA & NA & NA \\
\hline SNOX & NA & NA & NA & NA & NA & NA & NA & NA & NA & NA & NA & NA \\
\hline Springerville & $\begin{array}{l}0.1 \\
0.3 \\
0.2\end{array}$ & 0.2 & NA & NA & NA & NA & NA & NA & NA & NA & NA & NA \\
\hline Yates & NA & NA & NA & NA & NA & NA & NA & NA & NA & NA & NA & NA \\
\hline
\end{tabular}

$\mathrm{NA}=$ not available, $\mathrm{ND}=$ not detected, $"$ stack concentrations at Cardinal in pCi/dscm @ $3 \% \mathrm{O}_{2}$. 